\title{
Le littoral de la Tunisie, étude géoarchéologique et historique
} (1987-1997). La céramique

\author{
Michel Bonifay, Claudio Capelli, Thierry Martin, Maurice Picon, Lucy Vallauri
}

\begin{abstract}
Following the monograph published in 2004 by H. Slim, P. Trousset, R. Paskoff and A. Oueslati, Le littoral de la Tunisie (Coll. Études d'Antiquités africaines), this study presents the pottery collected on the 210 settlements registered during the 1987- 1997 coastal survey of Tunisia. This study comprises four chapters :

I. Antic pottery (M. Bonifay) provides some information about chronology of the settlements, regional typology and economic activities.

II. Physicochemical analysis (M. Picon) testify the importation in Tunisia of Gaulish wares from Savoy.

III. Petrology (Cl. Capelli) permits to caractérise several amphoras workshops along the Tunisian coastline.

IV. Islamic pottery (L. Vallauri) gives some precision about vessels production and importation in Ifriqiya from 9th to XVIIIth c.
\end{abstract}

\section{Résumé}

En complément de la monographie publiée en 2004 par H. Slim, P. Trousset, R. Paskoff et A. Oueslati, Le littoral de la Tunisie (Coll. Études d Antiquités africaines), cette étude présente les céramiques recueillies, de 1987 à 1997 , sur les 210 sites archéologiques du programme d'étude du littoral tunisien. Cette étude comprend quatre parties :

I. Les céramiques antiques (M. Bonifay) permettent de préciser la chronologie des sites, de différencier les faciès régionaux et de s'interroger sur la nature des activités économiques.

II. Les analyses physico-chimiques (M. Picon) prouvent l'importation en Tunisie de céramiques d'origine savoyarde.

III. Les analyses pétrographiques (Cl. Capelli) permettent de caractériser plusieurs ateliers d'amphores africaines répartis sur le littoral.

IV. Les céramiques d'époque islamique (L. Vallauri) apportent des précisions sur la production et les importations de vaisselles en Ifriqiya du IXe au XVIIle s.

\section{Citer ce document / Cite this document :}

Bonifay Michel, Capelli Claudio, Martin Thierry, Picon Maurice, Vallauri Lucy. Le littoral de la Tunisie, étude géoarchéologique et historique (1987-1997). La céramique. In: Antiquités africaines, 38-39,2002. pp. 125-202. doi : 10.3406/antaf.2002.1355

http://www.persee.fr/doc/antaf_0066-4871_2002_num_38_1_1355

Document généré le 19/11/2015 
BY: $\$$ creative 


\title{
LE LITTORAL DE LA TUNISIE, ÉTUDE GÉOARCHÉOLOGIQUE ET HISTORIQUE (1987-1997) \\ LA CÉRAMIQUE
}

\author{
Michel BONIFAY*, Claudio CAPELLI**, Thierry MARTIN***, Maurice PICON**** \\ et LuCy VALLAURI****
}

\begin{abstract}
Mots-clefs : prospections du littoral tunisien : études céramologiques; céramique à vernis noir, sigillée italique, sud-gauloise, bispanique, luisante, dérivée-des-sigillées paléocbrétiennes, sigillées africaines ; lampes; céramique culinaire, commune, modelée; amphores; ateliers ; chronologie ; faciès géographiques; analyses physico-chimiques, pétrographie ; céramiques d'époque islamique.

Key words : Coastal Survey of Tunisia : ceramic report; Black-glazed wares, Samian wares (Italic, South-gaulish, Hispanic ones), "Lucente " ware, South-gaulish grey ware, African Red Slip ware ; Lamps ; Cooking wares, Plain wares, Hand-made wares ; amphoras; Workshops; Chronology ; Regional typology; Physicochemical Analysis, petrography; Islamic wares.
\end{abstract}

Résumé : En complément de la monographie publiée en 2004 par H. Slim, P. Trousset, R. Paskoff et A. Oueslati, Le littoral de la Tunisie (Coll. Études d'Antiquités africaines), cette étude présente les céramiques recueillies, de 1987 à 1997 , sur les 210 sites archéologiques du programme d'étude du littoral tunisien. Cette étude comprend quatre parties :

I. Les céramiques antiques (M. Bonifay) permettent de préciser la chronologie des sites, de différencier les faciès régionaux et de s'interroger sur la nature des activités économiques.

II. Les analyses physico-chimiques (M. Picon) prouvent l'importation en Tunisie de céramiques d'origine savoyarde.

III. Les analyses pétrographiques ( $\mathrm{Cl}$. Capelli) permettent de caractériser plusieurs ateliers d'amphores africaines répartis sur le littoral. $I V$. Les céramiques d'époque islamique ( $L$. Vallauri) apportent des précisions sur la production et les importations de vaisselles en Ifriqiya du ix" au XVIIr's.

\begin{abstract}
Following the monograph published in 2004 by H. Slim, P. Trousset, R. Paskoff and A. Oneslati, Le littoral de la Tunisie (Coll. Etudes dintiquités africaines), this study presents the pottery collected on the 210 settlements registered during the $1987-$ 1997 coastal survey of Tunisia. This study comprises four chapters :

I. Antic pottery (M. Bonifay) provides some information about chronology of the settlements, regional typology and economic activities.

II. Physicochemical analysis (M. Picon) testify the importation in Iunisia of Gaulish wares from Savoy.

III. Petrology (Cl. Capelli) permits to caracterise several amphoras workshops along the Tumisian coastline.

IV. Islamic pottery (L. Vallauri) gives some precision about vessels production and importation in Ifriqiya from 9 th to XVIIth $c$.
\end{abstract}

\footnotetext{
* CNRS, Centre Camille Jullian (limR 6573), MMSH, 5 rue du Château de l'Horloge, BP 647. F-13094 Aix-en-Provence cedex 2.

- Università degli Studi di Genova, Dipartimento per lo Studio del Territorio e delle sue Risorse (DIP.TE.RIS.), Corso Europa 26, I-16132 Genova.

“.' Chercheur associé à l'Lnité toulousaine d'Archéologie et d'Histoire (CNRS, UMR 5608), 4 bd de la Mairie, 81200 Aussillon.

.... CNRS, Archéométrie et archéologie (UMR 5138), MOM, Maison de l'Orient Méditerranéen, 7. Rue Raulin. 69365 Lyon.

..... CNRS, Laboratoire d'Archéologie Médiévale Méditerranéenne (LMR 6572), MMSH, 5 rue du Château de l'Horloge, BP 647, F-13094 Aix-enProvence cedex 2.
} 
La documentation rassemblée dans le présent article $^{1}$ est issue d'un programme de prospection extensive des côtes de la Tunisie, depuis la frontière libyenne jusqu'à la frontière algérienne, conduit de 1987 à 1997 par Hédi Slim, Pol Trousset, Roland Paskoff et Ameur Oueslati. Ce programme pluridisciplaine, à la fois géomorphologique et archéologique, avait un double objectif : du point de vue des géographes, mettre en évidence les transformations de l'environnement littoral depuis l'Antiquité et, du point de vue des archéologues, recenser les vestiges d'installations antiques liées à l'exploitation de la mer.

Le catalogue des sites ainsi que les principales conclusions d'ordre historique, archéologique et géomorphologique tirées du programme d'étude du littoral tunisien ont fait l'objet d'une publication monographique synthétique ${ }^{2}$. En complément de cette publication, on trouvera ci-après le dossier consacré aux céramiques recueillies au cours de ces prospections.

\section{I - LA CÉRAMIQUE ANTIQUE}

(par Michel BONIFAY ${ }^{3}$, avec une contribution de Thierry MARTIN et la collaboration de Nejib BEN LAZREG $^{4}$ et Ali DRINE ${ }^{5}$ ).

1. Des impératifs éditoriaux nous contraignent à publier isolément cette étude qui devait initialement constituer le chapitre VI de la monographie consacrée aux résultats du programme d'étude du littoral tunisien et dont la parution, contre notre volonté trop longtemps différée, est maintenant effective dans la collection Études d'Antiquités Africaines. Malgré quelques révisons partielles en 2001 et 2003, l'essentiel du texte et des illustrations date de 1997.

2. Slim H., Trousset P. et al., Le littoral de la Tunisie, Paris, CNRS Éditions, 2004.

3. Ce texte n'aurait pu être écrit sans la collaboration active de nombreux collègues tunisiens qui nous ont très libéralement fait partager leur grande connaissance des régions dont ils ont la charge dans le cadre de leurs activités au sein de l'Institut National du Patrimoine (INP) et nous ont permis d'avoir accès au matériel de leurs fouilles et prospections. Je souhaite remercier tout particulièrement : Mmes Aïcha Ben Abed, Nabiha Jeddi, Neila Ouertani et Latifa Slim, MM. Samir Aounallah, Nejib Ben Lazreg, Ali Drine et Tahar Ghalia.

4. Chercheur à l'INP. Nejib Ben Lazreg a participé aux campagnes 1989 et 1990 de prospection (région du Sahel).

5. Chercheur à l'INP. Ali Drine a participé à la campagne 1990 de prospection (région de Zarzis et golfe de Gabès).
L'attention portée à la céramique ${ }^{6}$, dans le cadre de cette étude géoarchéologique et historique du littoral tunisien, avait un triple objectif. Tout d'abord, la céramique devait être utilisée comme outil de datation : c'est elle qui a fourni l'essentiel de la chronologie des sites recensés (Tabl. I). Rencontrée sur près de 200 sites $^{7}$ du sud au nord de la côte tunisienne (fig. 1-3), la céramique devait permettre ensuite de reconstruire les différents faciès régionaux. La céramique, enfin, était susceptible de nous renseigner sur la nature des activités des habitants du littoral, en recherchant notamment celles liées à l'exploitation de la mer (fig. 23).

\section{1 - La céramique, instrument de datation}

\section{A - Métbode utilisée}

La prospection extensive et itinérante d'un grand nombre de sites dans un délai relativement $\operatorname{court}^{8}$ n'a pas offert les conditions d'un ramassage systématique des céramiques. Les tessons ont été généralement laissés in situ, après avoir été identifiés et enregistrés ; seuls ont été récoltés ceux nécessitant une investigation plus poussée ou méritant d'être dessinés'), dont une petite partie seulement, enfin, a été exportée en France pour expertise complémentaire ${ }^{10}$. Ainsi, on ne s'étonnera pas de ne trouver aucune étude statistique. Par ailleurs le but avoué du programme étant de recenser les sites occupés durant l'antiquité, les traces d'occupation préhistorique n'ont pas été prises en compte et les céramiques d'époque islamique n'ont été qu'occasionnellement recueillies.

6. Dont un premier aperçu est donné dans : BONIFAY M., OlESLATI A., Paskoff R., Slim H. et Trolisset P., Programme tuniso-français d'étude du littoral de la Tunisie (bilan des travaux 1987-1990), BTravTun, 5, janvier-juin 1990, p. 109-112.

7. 170 des 210 sites repérés ont livré des témoins céramiques.

8. Quatre missions d'un mois de 1987 à 1990, complétées par une mission complémentaire d'une semaine en 1993. Les échantillons des campagnes 1987 et 1988 ont été inventoriés par Roger Guéry ; j’ai personnellement participé aux trois dernières campagnes.

9. Conservés dans divers dépôts de fouilles de l'Institut National du Patrimoine.

10. Actuellement conservés au Centre Camille Jullian (CNRSUniversité de Provence), MMSH, 5 rue du Château de l'Horloge, BP 647, 13094 Aix-en-Provence Cedex 02. 


\section{B - Validité des résultats}

Nous avons regroupé sur le tableau I les propositions de datation obtenues sur chaque site par l'examen des céramiques. On doit, une nouvelle fois, $s$ 'interroger sur la valeur historique réelle de telles indications, issues non pas de fouilles mais de prospections de surface. Il est nécessaire, afin de relativiser l'exploitation qui pourrait être faite ultérieurement de ces résultats, d'expliquer ici les trois obstacles auquel nous nous sommes heurtés dans l'interprétation des données.

a) L'identification des céramiques. L'enregistrement rapide des tessons de céramique sur chaque site risque d'avoir privilégié les catégories bien connues, notamment les sigillées africaines. L'époque punique a sans doute beaucoup souffert de cette situation : l'auteur de ces lignes ${ }^{11}$ connaît mal le matériel d'époque punique, beaucoup d'éléments significatifs ont pu lui échapper. En revanche, bénéficiant d'une expérience acquise sur la rive nord de la Méditerranée, les productions gauloises et les amphores orientales tardives ont été facilement identifiées ce qui surévalue peut-être leur représentation par rapport à d'autres importations qui n'auront pas été repérées. Il faut donc prendre en compte la part de subjectivité qui a pu influencer le ramassage des céramiques lors de la prospection.

b) La datation des céramiques. Malgré d'incessants progrès, la datation des céramiques reste un exercice difficile. Dans ce domaine, où les données sont de plus en plus nombreuses mais de moins en moins maitrisables, le consensus n'est pas toujours de mise : il n'est pas rare de trouver, dans divers ouvrages récents, des datations différentes pour une catégorie identique. On gardera à l'esprit que les datations proposées ici sont fondées sur des références (généralement mentionnées en note) dans le choix desquelles entre également une part de subjectivité.

c) L'utilisation des cêramiques issues de prospections de surface. Je suis personnellement sceptique sur le bien-fondé d'une utilisation sans réserve des céramiques recueillies en surface pour dater l'occupation de sites enfouis. La prospection de sites littoraux, où un certain nombre de coupes stratigra-

11. Iont čétait, en 1989 , la première expérience africaine. phiques sont naturellement taillées par la mer, rend bien compte de ce problème. Nous prendrons deux exemples :

- Les sites de Dagla et Mraïssa (sites nos 164-165) présentent des affleurements de maçonneries (harpes d'opus africanum) complètement ensablées et recouvertes d'une maigre végétation arbustive, sans aucune trace de mise en culture. Les tessons recueillis en surface sont rares et datent en général de l'Antiquité tardive. Or ces deux sites sont placés en arrière d'une falaise vive, en cours d'érosion, où sont visibles les murs, les sols superposés et de nombreux fragments de céramiques qui couvrent toute l'occupation du site, de l'époque punique à la fin de l'antiquité. Cet exemple est des plus banals et on objectera avec raison que l'importance du site (grandes agglomérations dont une citée dans les textes) interdisait a priori de conclure sur une seule occupation tardive au simple examen des tessons de surface. Mais s'il s'était agi d'un site de moindre importance, anonyme, et sans coupe de terrain, qu'aurions-nous inscrit sur le tableau I?

- Les fouilles entreprises sur l'usine de salaison de Nabeul (site $n^{\circ} 135$ ) par Jean-Pierre Darmon en 196566 et que nous avons poursuivies de 1995 à $2002^{12}$, ont révélé une stratigraphie complexe qui s'échelonne du ve s. av. J.-C. jusqu'à la fin du VII ${ }^{e}$ s. ap. J.-C. L'usine de salaison n'occupe qu'un créneau de deux siècles dans cette chronologie, vraisemblablement entre la deuxième moitié du I ${ }^{\text {er }} \mathrm{s}$. ap. J.-C. et, pour l'essentiel, le milieu du III $^{\mathrm{e}} \mathrm{s}$. Ici, pas de falaise, on est en présence d'une côte basse. Que voit-on alentour, à la surface des anciennes parcelles cultivées? Des murs affleurent à la rupture de pente vers la mer, avec des enduits de béton de tuileau qui trahissent, comme lors des premières interventions, la présence d'installations de salaison. Le matériel céramique qui jonche les sols, remonté par les labours, date de l'Antiquité tardive (réoccupation des ruines de l'usine) ou du III' s. (abandon de l'usine). Ainsi, les ramassages de céramiques non seulement oblitèrent complètement une phase d'occupation du site profondément enfouie (l'époque punique) mais ils ne sont également d'aucune utilité pour dater les structures affleurantes. De surcroît, selon un phénomène bien connu en archéologie, les céramiques contemporaines de

12. Sim I.., BOnflay M. et Trotsset P., L'usine de salaison, 1999. 
l'utilisation des bâtiments sont rares même au sein du matériel issu de la fouille; en fait, seule l'étude stratigraphique permet de dater la construction et l'abandon de l'usine.

Il faut donc rester extrêmement prudent quant à l'interprétation des données contenues dans le tableau I. Un traitement statistique des céramiques recueillies en prospection ne serait à mon sens d'aucun secours : s'il peut apporter des informations sur la diffusion géographique de telle ou telle production, il n'a guère de sens en termes de chronologie. Dans le cas extrême de sites peu érodés ou n'ayant jamais subi de mise en culture, j'estime qu'il faut accorder autant d'importance à un tesson de sigillée africaine $\mathrm{A}$ du II $\mathrm{e}$ s. qu'à un lot important de sigillée africaine $\mathrm{D}$ du $\mathrm{v}^{\mathrm{e}} \mathrm{s}$.

\section{C - Interprétation des résultats}

Ces réserves étant faites, quels enseignements peut-on tirer de la chronologie, même imparfaite, des 210 sites recensés sur le littoral tunisien? On remarque tout d'abord que quelques zones géographiques se distinguent par leur homogénéité :

a) Une première zone (sites nos 1 à 29) (fig. 1) correspond au sud du golfe de Gabès et comprend Jerba et la côte tripolitaine jusqu'à la frontière libyenne. Ici, la majorité des sites paraît occupée à l'époque romaine, sans beaucoup de traces de l'Antiquité tardive ou de l'époque punique : l'un des sites majeur, l'agglomération de Zoukhis ou Fisida $\left(\mathrm{n}^{\circ} 1\right)$ en est un exemple très caractéristique. Les seules exceptions notables sont situées en bordure de la Bahiret el Biban avec un ensemble résidentiel d'époque vandalo-byzantine (site $n^{\circ} 5$ ) et un village de pêcheurs préromain (site $n^{\circ} 7$ ), ou bien sur l'île de Jerba avec des agglomérations importantes à occupation longue. Le site 29, également occupé jusqu'à l'époque byzantine, est situé au débouché de la Mer de Bou Grara.

b) Au nord du golfe de Gabès, l'inflexion de la côte en direction du Nord-Est (sites nos 45 à 55) (fig. 1) nous met en présence d'une série de sites de l'Antiquité tardive, signalés par des lieux de culte chrétiens (basiliques d'Ad Oleastrum, site $\mathrm{n}^{\circ} 45$, et de Macomades-Iunci, site $\mathrm{n}^{\circ} 54$ ) et surtout par un site érémitique insulaire (monastère de Saint Fulgence sur un îlot des Kneiss, site $n^{\circ} 50$ ). Rien d'étonnant, en conséquence, à ce que la céramique tardive domine, même sur des sites à occupation longue.

c) La portion de côte comprise -lato sensu- entre Salakta et Mahdia (sites nos 91 à 104) (fig. 2) est jalonnée de sites d'époque romaine, même si certains sont encore fréquentés à l'époque byzantine - deux villes ont une occupation très longue : Sullectbum et Mahdia - tandis que la côte du Sahel comprise entre Thapsus et Monastir (sites nos 105 à 116) compte une série de sites à occupation tardive dominante, même sur des villes à occupation longue comme Thapsus ou Leptiminus (sites $\mathrm{n}^{\mathrm{os}} 105$ et 110).

d) Le fond du golfe d'Hammamet (sites nos 124 à 131) (fig. 2) est caractérisé par une série de sites occupés à l'époque vandale ou byzantine, mais il n'est pas exclu que beaucoup trouvent leur origine au début de l'Empire (sites nos 127 bis et 130). Au-delà de Pupput et de Neapolis (sites nos 132 et 134), on va trouver, dans les lagunes de Korba (sites nos 138 à 148), un ensemble d'installations à occupation tardive IVe-VII $\mathrm{s}$., liés, semble-t-il, au développement des activités halieutiques.

e) Le nord du Cap Bon et le golfe de Tunis jusqu'à Bizerte (sites nos 155 à 188) (fig. 2-3) sont marqués par la densité des témoins d'époque punique ancienne. Ce phénomène est peut-être dû à l'attraction des métropoles de Carthage et Utique.

f) Il faut mettre à part le cas des îles Kerkennah (sites nos 57 à 67) (fig. 1), Kuriate (site n 114) (fig. 2) et de La Galite (site $n^{\circ} 210$ ) qui comportent des sites à occupation longue (par exemple Cercina, site $\mathrm{n}^{\circ} 61$ ) et où les témoins d'époque punique sont abondants.

Au-delà de ces observations de détail, le phénomène le plus marquant est celui de la prédominance des contextes tardifs. Certes, beaucoup de sites occupés durant l'Antiquité tardive l'ont été également plus tôt. Mais le simple fait que ces sites soient encore habités à l'époque byzantine et que d'autres s'y ajoutent au même moment constitue l'un des principaux acquis chronologiques de notre prospection. Cette constatation n'est pas sans comparaison dans le monde méditerranéen. On connaît par exemple le développement important des villes côtières de Cyrénaïque, telle Apollonia qui sous le nom de Sousouza supplante 


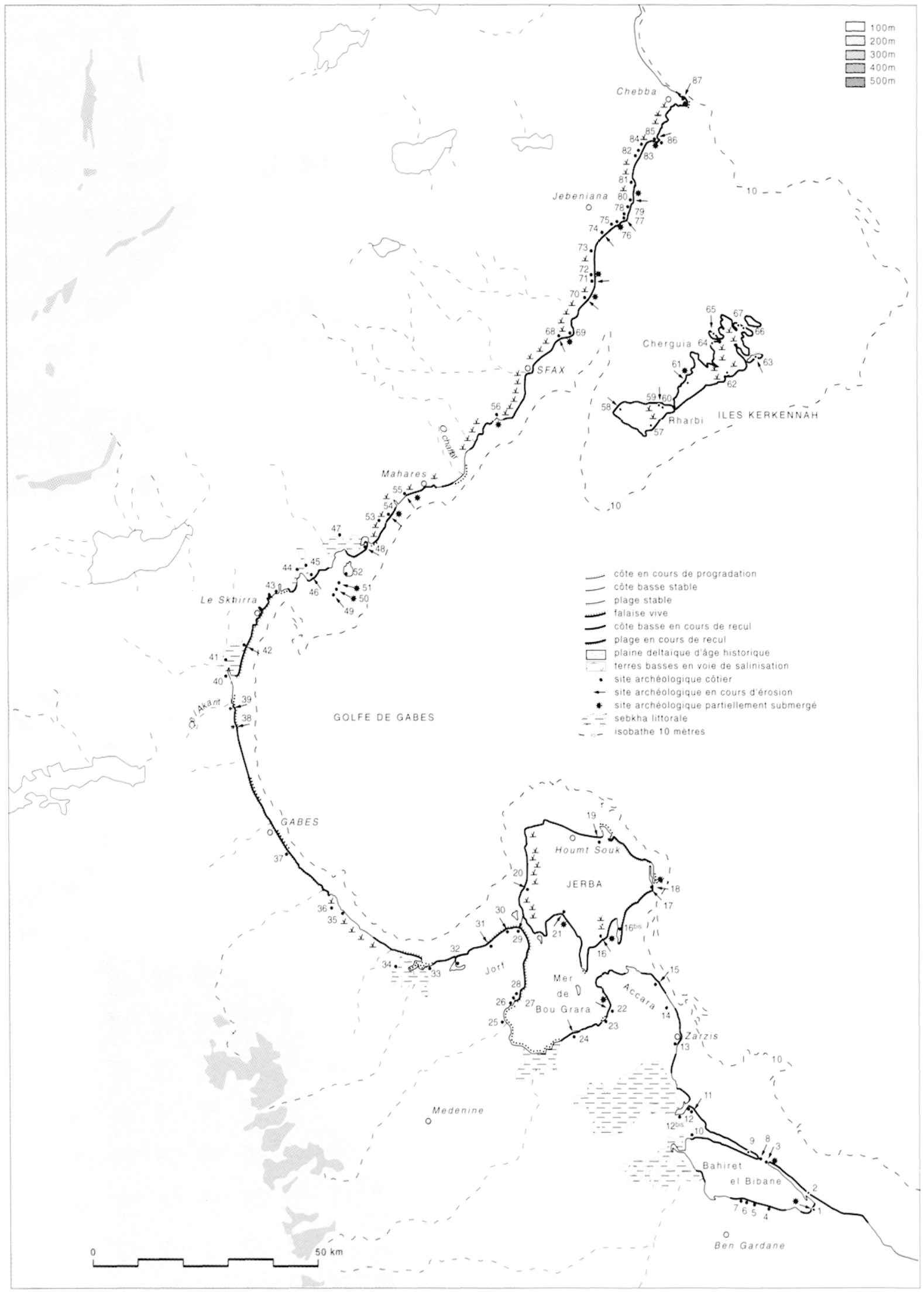

Fig. 1 : Le littoral tunisien. Carte des sites repérés. Tronçon Libye - La Chebba. (J. Lenne/V. Dumas) 


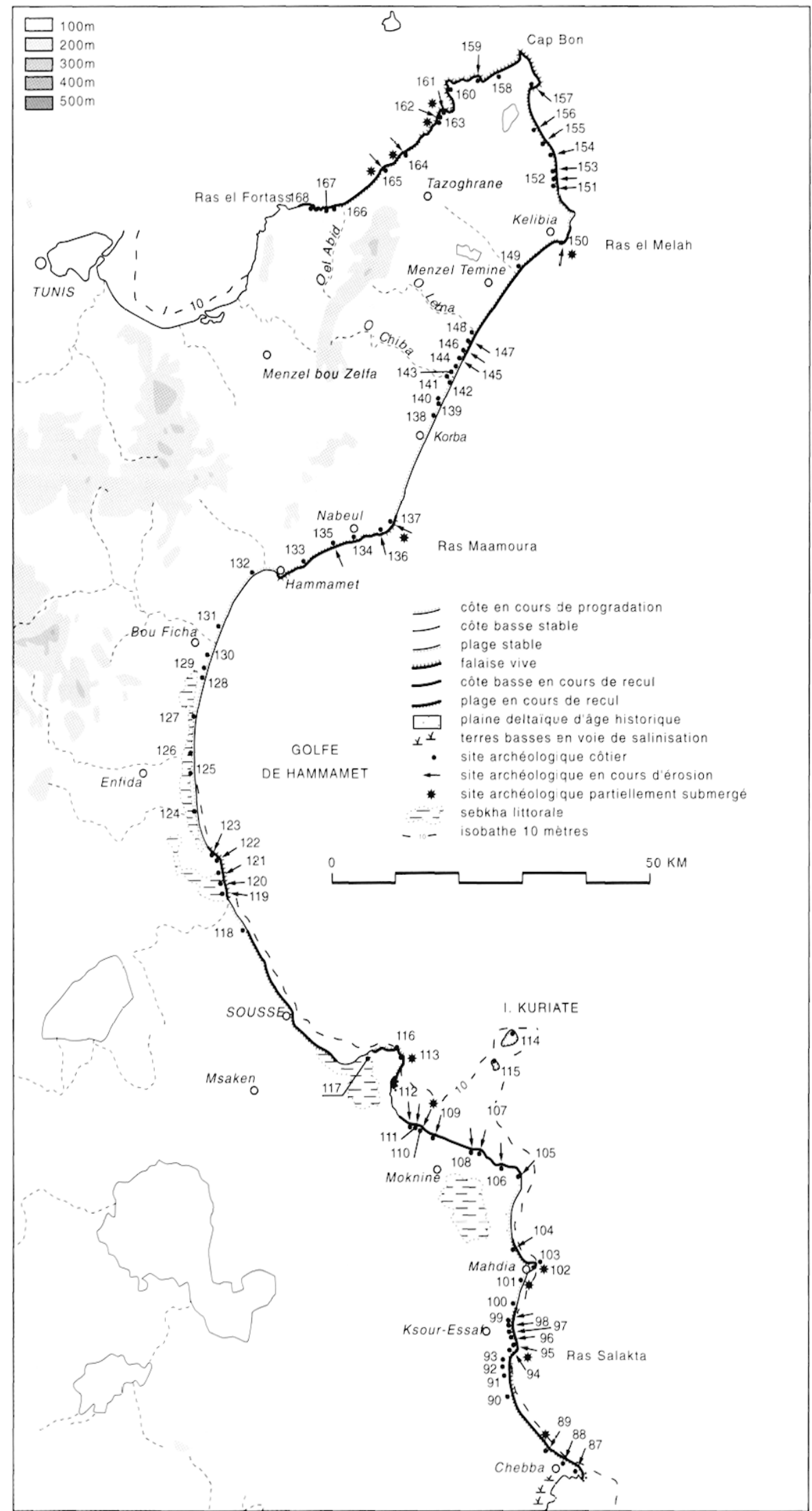

Fig. 2 : Le littoral tunisien. Carte des sites repérés. Tronçon La Chebba - Ras el Fortass. (J. Lenne/V. Dumas) 

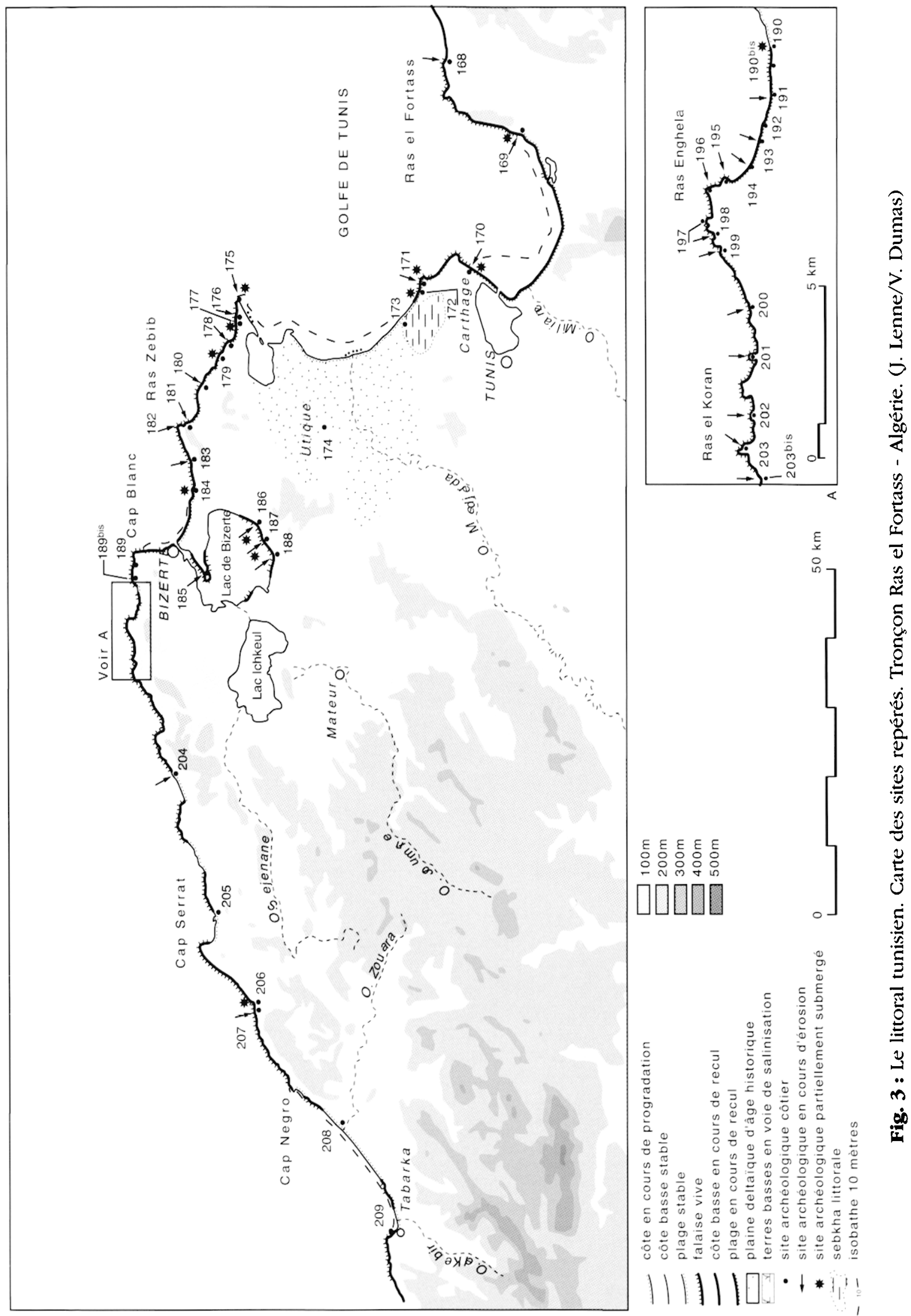
Cyrène comme capitale de la Libye Pentapole au courant du $\mathrm{v}^{\mathrm{e}} \mathrm{s}$., mais on citera également l'occupation tardive des îles proches du littoral et des zones lagunaires en Égypte ${ }^{13}$, en Vénétie ${ }^{14}$ ou en Camargue ${ }^{15}$, pour ne citer que quelques exemples. On a cherché parfois à expliquer cette densité de l'occupation du littoral par des raisons d'ordre sécuritaire. Mais on doit également constater que les capacités économiques du littoral restent importantes durant l'Antiquité tardive et qu'elles connaissent peut-être un nouvel élan favorisé par leur position géographique, notamment à l'époque byzantine, si l'on en juge par la diffusion des amphores et des vaisselles africaines des $\mathrm{VI}^{\mathrm{e}}$-VII ${ }^{\mathrm{e}} \mathrm{s}$. dans la partie du bassin méditerranéen restée ou repassée sous l'influence de Constantinople (voir infra).

\section{2 - Étude typologique et faciès régionaux}

\section{A - La vaisselle et les lampes}

\section{Céramiques à vernis noir (fig. 4)}

Alors que la céramique attique est abondante à Carthage ${ }^{16}$, peu nombreuses sont les céramiques à vernis noir antérieures à la deuxième guerre punique recueillies lors de nos prospections ; on supposera que cette rareté est due à la nature superficielle de nos investigations ${ }^{17}$. Les deux fragments illustrés ${ }^{18}\left(\mathbf{n}^{\mathbf{o s}} \mathbf{1}\right.$ et 2) ne sont probablement pas d'origine attique ${ }^{19}$ et présentent déjà des caractéristiques morphologiques propres aux exemplaires du Ive $s$. : lèvre légèrement

13. Jaritz H. et al., Pelusium, 1994, p. 153 et 155.

14. LFCIEJEWICZ L., TABACZYNSKA E. et TABACZINSKI St., Torcello, 1977.

15. Landuré C. et Pasqualini M., Delta du Rhône, 1997, p. 10.

16. MORFI. J.-P., Vernis noir de Carthage-Byrsa, 1982, p. 44 : " la céramique attique est représentée à Byrsa par un nombre considérable de fragments, dont une proportion non négligeable remonte au V' s. "; CHFLlBI F., Vernis noir de Cartbage, 1992, p. 17 : " la céramique attique est, incontestablement, la poterie d'importation dont le volume dépasse de loin celui de toutes les autres céramiques à vernis noir parvenues à Carthage avant la cleuxième guerre punique. ”

17. Les fouilles de l'usine de salaison de Nabeul (Sum L., BONIFAY M. et Trolssert P., L'usine de salaison, 1999) ont permis de récolter plusieurs fragments de céramique attique dont un pied de coupe de la fin du $\mathrm{Vl}^{\mathrm{C}} \mathrm{s}$. avant $\mathrm{J}$.-C.

18. Provenant tous deux du même site $n^{\circ} 21$ : Guellala, sur l'île de Jerba.

19. Je remercie M. Jean-Paul Morel d'avoir bien voulu examiner nos échantillons. déversée du bolsal ${ }^{20}\left(\mathbf{n}^{\circ} \mathbf{1}\right)$ et profil à double courbure du skyphos ${ }^{21}\left(\mathbf{n}^{\circ} \mathbf{2}\right)$.

Présente sur une quinzaine de sites $^{22}$, la céramique à vernis noir dite " campanienne " est plus fréquente. De fait, tout comme à Carthage ${ }^{23}$, les productions de Naples-Ischia (Campanienne A) dominent quantitativement : cependant, à côté d'éléments "classiques ", par exemple le fond $\mathbf{n}^{\circ} \mathbf{6}$ décoré de palmettes radiales et de guillochis, d'autres tessons, tels ces décors de feuilles simplifiées visibles sur les fonds nos $\mathbf{7}$ et $\mathbf{8}$, évoquent plutôt des contextes postérieurs à la chute de Carthage ${ }^{24}$. Les autres productions sont attestées de manière plus ténue $\left(\mathrm{n}^{\circ} \mathbf{4}\right.$ ?) ; celles d'origine punique, d'une qualité proche de la classe Byrsa $401^{25}$, règnent quasiment sans partage dans un contexte homogène du milieu du III $s$. à Nabeul ${ }^{26}$.

Une troisième catégorie de céramique à vernis noir ou brun apparaît à trois reprises ${ }^{27}$, celle des bols hellénistiques à reliefs. Le fragment illustré $\mathbf{n}^{\circ} \mathbf{3}$ paraît d'origine ionienne.

\section{Catalogue}

[N.B. : le $\mathrm{n}^{\circ}$ de site est indiqué par les trois premiers chiffres du $n^{\circ}$ d'inventaire]

-1 : céramique à vernis noir (non attique : origine ?), bolsal (?) ; pâte orange-rose, compacte, assez granuleuse, avec de grandes vacuoles; vernis noir à reflets métalliques, coups de brosse à l'int. (Inv. 021.03)

- 2 : céramique à vernis noir (non attique : origine ?), skyphos ; pâte beige-rose, granuleuse ; vernis noir mat, écaillé. (Inv. 021.07)

-3 : bol hellénistique à reliefs, forme SIEBert G., Bols à reliefs, 1980, fig. 9 ; décor en trois zones (de bas en haut :

20. Sparkes B. et TalCotT L., Black and Plain Pottery, 1970, p. 107.

21. Ibid., p. 85.

22. Sites nos 7, 16, 19, 21, 59, 61, 64, 66, 72, 164, 167, 169, 185.

23. MOREL J.-P., Vernis noir de Carthage-Byrsa, 1982, p. 47 : a il ne paraît pas excessif d'affirmer que la $\mathrm{A}$ représente une bonne moitié, sinon plus, de la céramique à vernis noir (...) consommée à Carthage "; Chelbi F., Vernis noir de Carthage, 1992, p. 27 : “ à Carthage (...) le tesson de campanienne A ne disparaît pas du champ de vision...

24. MOREL J.-P., Vernis noir de Carthage-Byrsa, 1982, p. 49. Voir, par exemple, l'association sur l'épave de Punta Scaletta à Giannutri de la forme Lamboglia 27B de grand module et des décors de feuilles radiales simplifiées : Lamboglia N., Punta Scaletta, 1964, p. 244 et 246.

25. MOREL J.-P., Vernis noir de Carthage, 1986, p. 29.

26. Sum L., Bonifay M. et Trousset P., L'usine de salaison, 1999, fig. 23, no 1 à 4 .

27. Sites $n^{\text {os }} 16,61,66$. 
écailles aiguës, perles et pirouettes, étoiles carrées à huit branches), les deux inf. n'étant pas séparées par des filets (style d'Hera[ios] ?) ${ }^{28}$. (Inv. 061.09)

- 4 : céramique à vernis noir (Campanienne $C$ ?), apparentée à la forme Lamboglia 6 (?)29. (Inv. 061.07)

- 5: céramique à vernis noir (Campanienne A), forme Lamboglia $27 \mathrm{~B}$ de grand module (d. $24 \mathrm{~cm}$ ), type Morel 2825 e ; pâte orange-rose, granuleuse ; vernis noir, brillant, écaillé. (Inv. 007.01)

- 6 : céramique à vernis noir (Campanienne A); décor de palmettes radiales à l'int. d'un cercle de guillochis ; pâte orange-rose, granuleuse ; vernis noir, brillant, écaillé, trace rouge de doigt à l'ext. du pied. (Inv. 061.02)

- 7 : céramique à vernis noir (Campanienne A); décor de quatre feuilles radiales simplifiées ; pâte beige orangé, granuleuse ; vernis noir, brillant, écaillé, traces de doigt à l'ext. autour du pied. (Inv. 021.02)

- 8: céramique à vernis noir (Campanienne A); décor de feuilles radiales (?) simplifiées à l'int. d'un cercle de guillochis. (Inv. 061.08)

\section{Céramique sigillée italique (fig. 4)}

La sigillée italique est attestée sur 16 sites $^{30}$, avec des produits d'origine incertaine (majoritairement non arétins ?), déjà signalés ailleurs en Afrique et couvrant tout le $\mathrm{I}^{\mathrm{er}} \mathrm{s}$. de notre ère. Aux premières années du siècle doivent être attribués le timbre OPTATVS ( $\mathbf{n}^{\circ} \mathbf{1 0}$ ), bien diffusé en Espagne, en Gaule, à Rome et Ostie $^{31}$, à Ordona ${ }^{32}$, à Corinthe ${ }^{33}$, en Afrique (Tamuda, Cherchell, Carthage, Leptis Minus) ${ }^{34}$, ainsi que le timbre $L . P() P V D(E N S)\left(\mathbf{n}^{\circ} \mathbf{1 2}\right)$, connu à Rome ${ }^{35}$, à Ordona ${ }^{36}$ et en Afrique : Constantine et Dellys ${ }^{37}$, Uzita ${ }^{38}$. L'assiette de forme Conspectus 20 = Goudineau 39c ( $\mathbf{n}^{\circ}$ 9) est caractéristique du milieu ou de la deuxième moitié du $\mathrm{I}^{\mathrm{er}} \mathrm{s} .{ }^{39}$, tout comme vrai-

28. Latmonier A., Céramique bellénistique à reliefs, 1977, pl. 64, $\mathrm{n}^{\circ} 5298$, pl. 65, n०5319, pl. 66, n's 8784 et 5279.

29. BATs M., Olbia, 1988 , pl. $27, \mathrm{n}^{\circ} 823$.

30. Sites nos 12, 16, 21, 43, 47, 61, 83, 162, 163, 164, 165, 166, 167, 169, 181, 210.

31. OXÉ A. et COMFORT H., CVArr, 1968, p. 300.

32. VANDERHOEVEN M., Terre sigillée, 1988, p. 107.

33. HAYES J.W., South Stoa, 1973, p. 435 et 438, nos 4 et 56.

34. GLÉRY R., Marques de potiers IV/2, 1994, p. 100.

35. OXF́ A. et COMFORT H., CVArr, 1968, p. 304.

36. VANiderhofven M., Terre sigillée, 1988, p. 108.

37. GL'́r.RY R., Marques de potiers IV/2, 1994, p. 100, n" 476 et 477

38. VAN DER WERFF J.H., Uzila, 1982, pl. 65, nº 12. semblablement ${ }^{40}$ le timbre $C N . A T(E I) A R(R E T I N I)$ $\left(\mathbf{n}^{\circ} \mathbf{1 1}\right)$, déjà relevé à plusieurs reprises en Afrique : Constantine, Tiddis, Carthage, Tunis ${ }^{41}$ et Sabratha ${ }^{42}$.

\section{Catalogue}

- 9: céramique sigillée italique, type Conspectus 20.4 (forme Goudineau 39c); décor d'applique : Eros (?) à gauche, empâté ; pâte brun rouge, fine, sableuse ; vernis rouge carmin, brillant. (Inv. 021.06)

- 10 : céramique sigillée italique; timbre rectangulaire OPTA / TVS : CVArr 1176. (Inv. 061.01)

- 11 : céramique sigillée italique ; timbre in p. p. [C]N AT AR : CVArr 151: Pise (?) ${ }^{43}$. (Inv. 167.01)

- 12 : céramique sigillée italique ; timbre in p.p. [L] P.PVD : CVArr 1195, officine non arétine (?) ${ }^{44}$. (Inv. 099.05)

- 13 : céramique sigillée italique (?) ; timbre rectangulaire

(?) [...]E / [..]JON : indéterminé, peut-être CN. ATEI ERONI

(?) (CVArr 159.10 ?). (Inv. 061.02)

\section{Céramique sigillée du sud de la Gaule (fig. 4)}

Il a été démontré à plusieurs reprises que les importations de sigillée italique sont plus abondantes en Afrique jusqu'à une date tardive que celles des sigillées du sud de la Gaule ${ }^{45}$. Toutefois, même en quantité inférieure, ces dernières paraissent bien diffusées ${ }^{46}$ : on les rencontre sur un nombre équivalent ${ }^{47}$ de sites du littoral, avec quelques-unes des formes les plus fréquemment signalées en Afrique (Algérie $^{48}$; Tunisie ${ }^{49}$; Libye $\left.{ }^{50}\right)$ : Drag. 27 (nº 14),

39. KF.rick Ph.M., Sidi Krabish, 1985, p. 154.

40. COMFORT H., Late Ateius signatures, 1962.

41. GUF́RY R., Marques de potiers IV/1, 1992, p. 46.

42. COMFORT H., Signatures and decoration, 1982, p. 500, $n^{\circ} 64$.

43. KenRiCK Ph.M., Cn Ateius, 1997, p. 188.

44. GuÉRY R., Marques de potiers IV/2, 1994, p. 100.

45. SOREN D., Utica, 1978 , p. 110 : “ South Gallic wares are not very common at Utica while Arretine occurs in some quantity even in the period 50-75 A.D. when one would expect to find Gallic in abundance *. De manière plus générale : GLERY R. Marques de potiers I, 1979, p. 96 et note 8 (bibl.), et Gl.FRY R., Terra Sigillata gauloise en Afrique, 1982, p. 69 (tabl.) ; à Carthage, en dernier lieu : F(t.FORI) M.G. et Timis J., Fine Wares, 1994, p. 1.

46. Voir également DoR: J., Lybian Valleys Pottery, 1996, p. 323. tabl. 7 : la sigillée du sud de la Gaule est plus abondante que la sigillée italique dans l'arrière pays de la Tripolitaine.

47. Quinze sites : 4, 16, 19, 21, 41, 47, 64, 121, 127bis, 130, 164, 165 . $166,176,210$.

48. Glér.r R., Marques de potiers I, 1979, fig. 17.

49. BotiRgFols A., Sigillée de la Graufesenque en Afrique, 1986.

50. Surtout forme Drag. 27 : DORE J., Lybian Valleys Pottery. 1996. Tabl. 7 ; ARTHL:R P., Amphora Production, 1982, fig. 8, $\mathrm{n}^{\circ} 31$. 

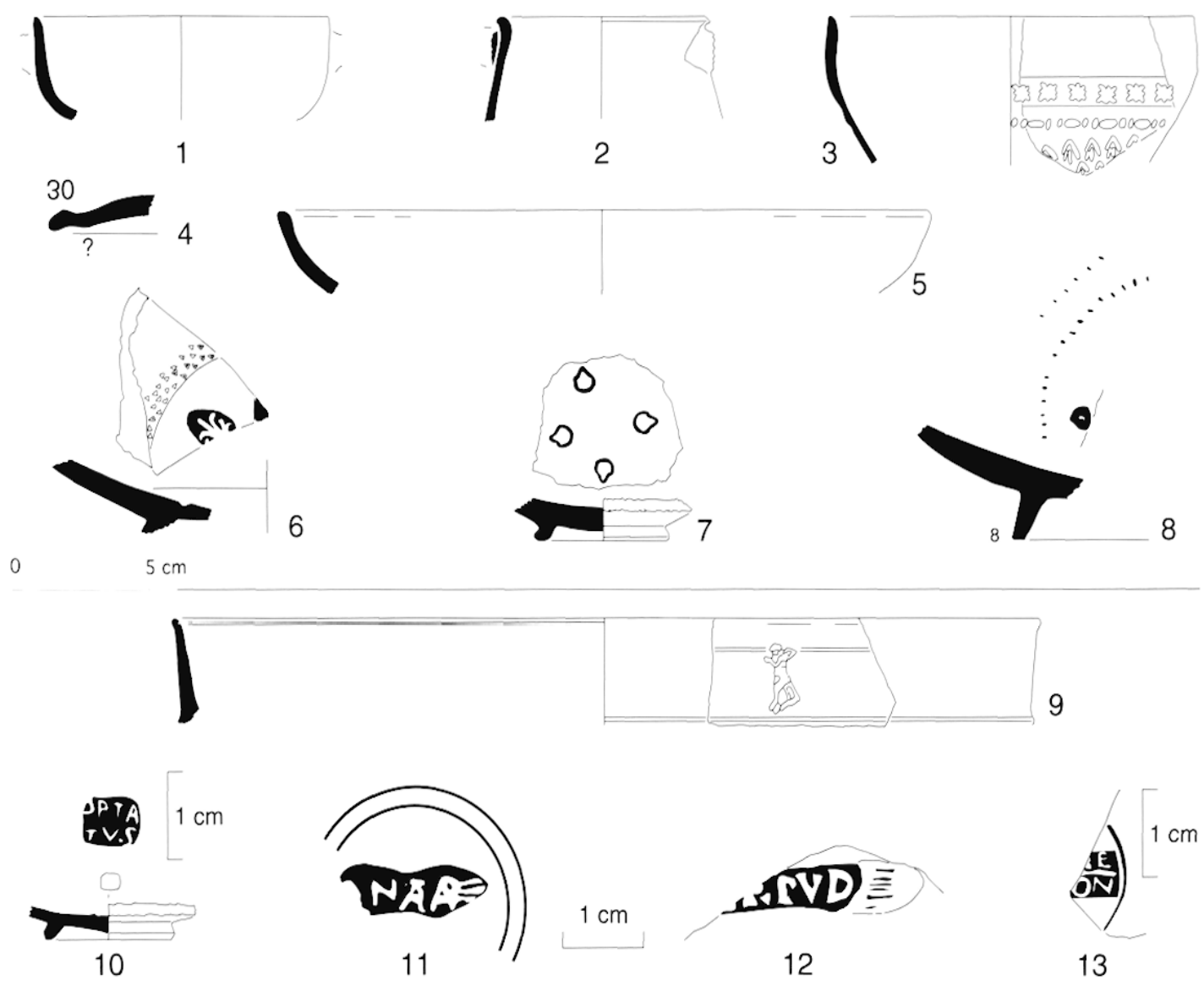

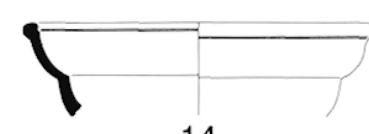

14

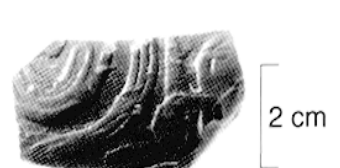

0 $5 \mathrm{~cm}$

17
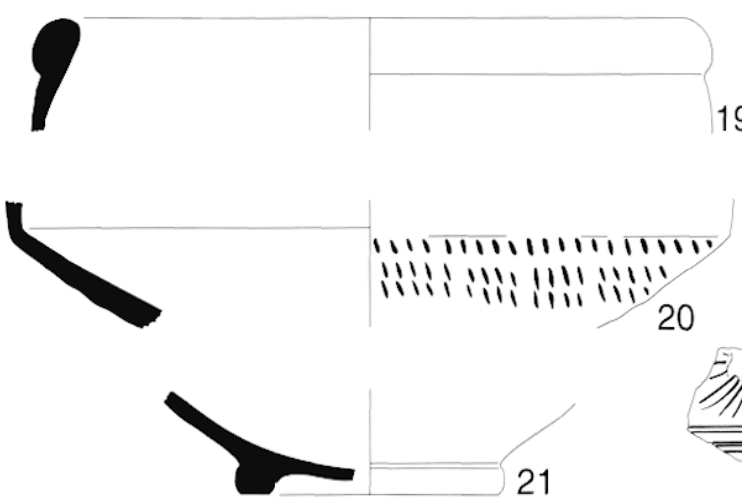

आ1 11111111110

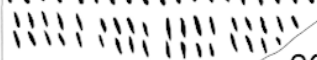

15

19
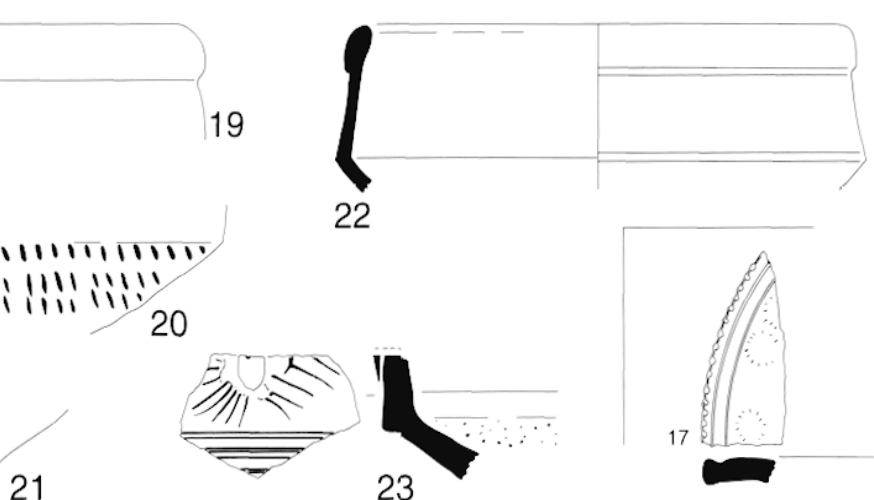

21

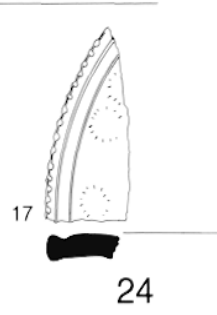

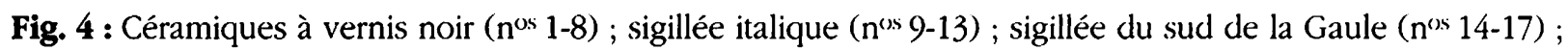
sigillée hispanique ( $\left.\mathrm{n}^{\circ} 18\right)$; sigillée claire gauloise "luisante " ( $\left.\mathrm{n}^{\circ \mathrm{s}} 19-23\right)$; sigillée tardive du sud de la Gaule (DS.P.) $\left(n^{\circ} 24\right)$. (M. Bonifay) 
Drag. 15/17 ( $\left.\mathbf{n}^{\circ} \mathbf{1 5}\right)$, Drag. $29\left(\mathbf{n}^{\circ} \mathbf{1 7}\right)$ et Drag. 37. L'origine rutène de ces produits est vraisemblable ; elle est sûre pour le timbre Passienus $\left(\mathbf{n}^{\circ} \mathbf{1 6}\right)$, attesté à La Graufesenque et très répandu, notamment en Afrique ${ }^{51}$, ainsi que pour les vases " marbrés " qui apparaissent en quantité non négligeable dans des contextes du milieu du I ${ }^{\mathrm{er}} \mathrm{s}$. à Nabeul ${ }^{52}$.

\section{Catalogue}

- 14 : céramique sigillée du sud de la Gaule, forme Dragendorff 27 ; pâte rouge brique, fine, compacte ; vernis rouge brillant. (Inv. 021.04)

- 15 : céramique sigillée du sud de la Gaule (?), forme Dragendorff $15 / 17$; pâte rouge brique, fine, compacte ; vernis rouge satiné. (Inv. 016.02)

- 16 : céramique sigillée du sud de la Gaule ; timbre PASSE[...] : Passienus, La Graufesenque, Néron-Vespasien (I.P.S., p. 227). (Inv. 016.11)

- 17 : céramique sigillée du sud de la Gaule, forme Dragendorff 29 (?) ; décor de rinceaux ; pâte rouge brique, fine, compacte ; vernis rouge brillant. (Inv. 016.05)

(M. B.)

\section{Céramique sigillée hispanique (fig. 4)}

Celle-ci n'est représentée que par un seul tesson $\left(n^{\circ}\right.$ 18). Il s'agit d'un fragment de bas de panse d'un Drag. 37 orné de médaillons circulaires séparés par des lignes tremblées verticales. Ce schéma décoratif, au demeurant des plus classiques, est caractéristique des productions hispaniques de la seconde moitié de la période flavienne et s'est prolongé sous les Antonins ${ }^{53}$. D'après la couleur de sa pâte et l'aspect de son vernis, et avec les réserves d'usage, il semble que l'origine de ce vase puisse être attribuée au centre producteur de Tricio (Tritium Magallum) dans la Rioja - ou à l'un de ses ateliers satellites (Bezares ?) plutôt qu'à la manufacture andalouse d'Andújar ${ }^{54}$.

51. GIERY R., Marques de potiers I, 1979, p. 70.

52. StuM L., BONIFAY M. et Trolssit P., Lusine de salaison, 1999. On note également un tesson de sigillée orientale A, forme Hayes 3.

53. Voir de multiples schémas décoratifs similaires dans MrzQlíri\% DE Catalan M.A., Terra Sigillata Hispanica, 1961, dans Garabito GOMEZ T., Alfares Romanos riojanos, 1978, de même que dans MAYET Fr., Céramiques sigillées bispaniques, 1984.

54. ROCA ROL:Mr.:s M., Sigillata bispánica, 1977 ; SOTOMAYOR M., Marcas y estilos, 1976 ; MAYtr Fr., Céramiques sigillées bispaniques, 1984, p. 35-57.
La découverte de cette pièce appelle, de notre part, un bref commentaire. Nul n'ignore, en effet, que les sigillées hispaniques ont été essentiellement diffusées à l'intérieur de la péninsule Ibérique et, dans une moindre mesure, en Maurétanie tingitane ${ }^{55}$. Ailleurs, celles-ci n'ont été que fort peu commercialisées si ce n'est, peut-être, dans le sud-ouest de la France, en Aquitaine méridionale, où quelques sites privilégiés du piémont pyrénéen, du Pays basque et des Landes en ont livrées dans des proportions parfois non négligeables $^{56}$. Il n'en va pas de même en Narbonnaise, où la présence de la sigillée hispanique se limite à quelques trouvailles isolées faites le long du littoral languedocien et provençal en milieu portuaire, notamment à Port-Vendres ${ }^{57}$, à Gruissan ${ }^{58}$ ou encore à Marseille ${ }^{59}$. En Italie, on observe la même indigence, comme le montrent les trouvailles ponctuelles effectuées à Luni ${ }^{60}$, à Ostie ${ }^{61}$ et à Rome ${ }^{62}$. L'Afrique du Nord déroge quelque peu à cette règle, dans la mesure où la Tingitane, nous l'avons dit, a été un temps assez régulièrement approvisionnée en vaisselle sigillée sinon par les potiers hispaniques, du moins par les negotiatores qui commercialisaient alors leurs productions. Le nombre élevé des estampilles originaires de Tritium Magallum et d'Andújar, rencontrées à Banasa, à Lixus, à Sala ainsi qu'à Volubilis, le prouve clairement ${ }^{63}$. Un phénomène analogue, mais d'une amplitude bien plus atténuée, a touché également la Césarienne et la Numidie, dont

55. Ml:zQtírIZ M.A., Terra Sigillata Ispanica, 1985, p. 111.

56. MrzQlírz dE CATAl.AN M.A., Aportaciones, 1960 ; Bol BE J., La Terra Sigillata bispanique, 1965, p. 20 ; MAYFT Fr., Expansion, 1969 ; ID., Céramiques sigillées bispaniques, 1984, I, p. 234-235; Mak'TIN Th., Un vase en terre sigillée hispanique, 2002. C'est notamment le cas de Lescar, l'antique Beneharmum, où, sur 1316 tessons de sigilléc étudiés par J.-M. Laporte en 1981, 234 ont une origine hispanique, soit $17,7 \%$ du total (LAPORTE J.-M., Céramique sigillée de Lescur, 1981, p. 124).

57. Il s'agit d'un fragment de panse d'une assiette Drag. 15/17 de Tricio, trouvé aux abords immédiats de l'épave "Port-Vendres I ", et conservé au dépôt de fouilles de Port-Vendres (Pyrénées-Orientales).

58. Sabrif́ R., Sigillée hispanique, 1981, fig. 101 ; à Narbonne : Ibid., p. 248, note 307 .

59. Martini:Z MuNilia C., Vaso de "terra sigillata " hispanica, 1950. 60. Frova A., Luni II, Tav. 109, $n^{\circ} 4$ et 5.

61. PALl.ARES Fr., Terra sigillata ispanica, 1963 ; CARANiJIN A. et PANFlla Cl., Ostia $I V$, p. 322 et tav. 80, n 594.

62. Glacillimi A. et Petricciont: V.. Roma. 1978, p. 88, n"m 359-360 : MARTIN A.N. Altre Sigillate, 1996.

63. BOLBE: J., Terra Sigillata bispanique, 1965 ; ROCA Rol MI: MS M., Sigillata bispánica, 1976, p. 104-105 ; MAYHT Fr., Céramiques sigillées hispanigues, 1984, I, p. 225. 227 et fig. 12. 
plusieurs sites, principalement côtiers, comme Cherchell, Tipasa ou Sétif, ou encore Oued Bibi, ont livré quelques échantillons de TS hispanique ${ }^{64}$. Enfin, il est à noter que cette pièce vient s'ajouter aux très rares vases sigillés d'origine hispanique identifiés jusqu'à présent en Proconsulaire, notamment à Carthage ${ }^{65}$ et tout récemment à Zama ${ }^{66}$.

\section{Catalogue}

- 18: céramique sigillée hispanique, forme Dragendorff 37 ; décor de médaillons circulaires séparés par des lignes tremblées verticales ; pâte rouge brique, fine ; vernis rouge carmin brillant. (Inv. 019.04)

(Th. M.)

\section{Céramique sigillée claire gauloise "luisante " (fig. 4)}

Si le commerce des céramiques gauloises du HautEmpire en Afrique et celui des céramiques africaines tardives en Narbonnaise sont des phénomènes bien connus, on manquait jusqu'à présent de données concernant un éventuel arrivage de vaisselles gauloises postérieures à la production de masse des ateliers rutènes des deux premiers siècles de notre ère. Dans ce domaine, les prospections du littoral tunisien apportent une documentation tout à fait inattendue avec la découverte d'un nombre non négligeable de tessons de sigillée claire rhodanienne du type "lucente " de Lamboglia ${ }^{67}$. Ces éléments permettent d'accroître considérablement la zone de diffusion de ces céramiques que l'on pensait limitée à "l'angle nord-occidental de la Méditerranée "68, les témoins les plus méridionaux ayant été récemment publiés à Belo ${ }^{69}$. Les analyses effectuées en laboratoire (Picon, infra) confirment que les productions rencontrées en

64. GUÉRY R., Trois vases de terra sigillata hispanique, 1971 ; GLtKY R., Marques de potiers I, 1979, p. 31-37 ; MAYFT Fr., Céramiques sigilleees hispaniques, 1984, I, p. 234-235 ; VERTET A., Ceramica romana imperial, 1961, p. 204-205 et fig. 1.

65. Hedrngl: B. et al., Die Frühe Terra Sigillata, 1999, p. 362-363, Abb. 25, Taf. I h, nos 309-310.

66. Fragment de panse d'une coupe hémisphérique Drag. 37 (renseignement aimablement communiqué par A. Ferjacui et A. Vernhet).

67. LavibogilaA N., Sigillata Chiara II, 1963, p. 163-179 ; PICON M. et DEsBAT A., Sigillée claire B et luisante, 1986 ; AA. VV., Céramiques tardives à revêtement argileux, 1986 ; PriknON J. et Chr., Portout, 1990.

68. Lavibogida N., Sigillata Chiara II, 1963, p. 163.

69. Botrgilols A. et MAYri Fr., Belo, 1991, p. 368-369.
Afrique proviennent d'ateliers voisins de celui de Portout (Savoie). Cet atelier est actif principalement dans la première moitié du $\mathrm{v}^{\mathrm{c}} \mathrm{s} .^{70}$; de fait, sur les sept sites $^{71}$ où elle est présente, la sigillée claire "luisante ", avec des formes typiques de cet atelier (type Pernon 37 à décor surpeint : $\mathbf{n}^{\circ} \mathbf{1 9}$, et type Pernon 40 à mufle de lion : $\mathbf{n}^{\circ} \mathbf{2 3}$ ), est associée à du matériel africain du ve $s$. (voir le détail dans le catalogue des sites).

\section{Catalogue}

- 19 : céramique sigillée claire " luisante ", forme Lamboglia $1 / 3$; pâte beige orangé ; engobe orange ext. avec traces de décors surpeints, violacé int. (Inv. 055.04)

-20 : céramique sigillée claire " luisante ", forme Lamboglia $1 / 3$; décor de guillochis ; pâte beige orangé ; engobe orange écaillé. (Inv. 033.01)

- 21 : céramique sigillée claire " luisante ", forme Lamboglia $1 / 3$ (?). (Inv. 004.01)

- 22 : céramique sigillée claire "luisante ", forme Lamboglia 1/3. (Inv. $055.01 ; n^{\circ}$ d'analyse : ACD 99)

-23 : céramique sigillée claire " luisante ", forme Lamboglia 45 ; bec verseur à mufle de lion. (Inv. $037.10 ; \mathrm{n}^{\circ}$ d'analyse : ACD 98)

\section{Sigillée tardive du sud de la Gaule (Dérivées-des- Sigillées Paléochrétiennes) (fig. 4)}

Cette céramique, issue d'ateliers languedociens ou provençaux $^{72}$, semble a priori mieux diffusée que la précédente puisqu'on en trouve des témoins au Maroc $^{73}$ et en Algérie ${ }^{74}$. Mais elle reste rare en Tunisie $^{75}$. Le seul tesson recueilli lors de nos prospections provient d'un site de la côte nord, en association avec des céramiques africaines du $\mathrm{v}^{\mathrm{e}} \mathrm{s}$.

70. Pernon J. et Chr., Portout, 1990, p. 214.

71. Sites $n \times 4,33,37,41,55,59,203$.

72. Le groupe aquitain est plutôt diffusé vers le nord de l'Europe : RigorR J., Sigillées Paléochrétiennes, 1968.

73. Jodin A. et Poxisich M., Céramique estampée du Maroc, 1960 , fig. 16 a et $\mathrm{c}$ : forme Rigoir 1, atelier languedocien?; AKERRAZ A. et al., Dchar Jdid, 1981-82, pl. XXI, n ${ }^{\circ} 79-148$ : forme Rigoir 6, atelier languedocien:

74. Barad: $\%$ J., Nouvelles fouilles, 1961, p. 130 et pl. V, $n^{\circ} 2$ : forme Rigoir 3, atelier provençal (?) ; d'autres tessons signalés à Cherchell et Rusguniae : RıciolR J. et Y., Dérivées-des-Sigillées Paléochrétiennes. 1987. note 15.

75. Au moins un tesson à Carthage : FLIFORI) M. et PFACOCK D.P.S., Avenue Bourguila, 1984, p. 225 et fig. 88, $\mathrm{n}^{\circ} 64$ : forme Rigoir 18. atelier marseillais. 


\section{Catalogue}

- 24 : Dérivée-des-Sigillées Paléochrétienne (DSP), forme Rigoir 3 ; décor de faux godrons et de rouelles sur le marli ; pâte grise sableuse ; engobe gris usé. (Inv. 204.01)

\section{Sigillées africaines}

Les tentatives de classement des différentes catégories de sigillées africaines ont fait couler beaucoup d'encre $^{76}$; leur caractère schématique et arbitraire a été à juste titre souligné ${ }^{77}$. Cependant, en l'absence d'un recensement systématique des ateliers et d'un inventaire précis de leurs productions, on en est encore réduit à utiliser ces distinctions tout en sachant qu'elles s'adaptent mal, parce qu'élaborées hors d'Afrique, à une réalité rendue beaucoup plus complexe par la proximité des lieux de fabrication. La prospection du littoral tunisien a été une nouvelle fois l'occasion de travailler sur la typologie et la diffusion de ces différents groupes.

\section{- Sigillée africaine $A$ (fig. 5)}

La sigillée africaine A est la seule production africaine dont on ne connaisse aucun atelier ; on hésite encore sur son origine géographique : nord de la Tunisie $^{78}$ et/ou Tunisie centrale ${ }^{79}$. Les prospections du littoral n'apportent rien de neuf dans ce domaine : cette céramique est présente sur 38 sites régulièrement répartis du sud au nord du littoral ${ }^{80}$ (cette faiblesse relative de représentation doit être interprétée à la lumière des considérations préliminaires sur les conditions de la prospection : voir supra). Au sein de cet échantillon réduit, dominent les formes de la production $\mathrm{A} 1\left(\mathbf{n}^{\circ} \mathbf{2 5}\right)$; celles de la production A2 ne sont attestées que sur trois sites ${ }^{81}$ où, dans deux

76. WAAGÉ F.O., Hellenistic and Roman Tableware, 1948 ; LAMBOGil.IA N., Sigillata Chiara I, 1958, et Sigillata Chiara II, 1963 ; SALOMONSON J.H., Étude sur la céramique, 1968 ; CARANIINI A., Ostia I, 1967-68 ; HaYes J.W., Late Roman Pottery, 1972, p. 289-292 ; CaRanDINI A. et al., Allante I, 1981 ; SCHICRING J.M., Terra Sigillata Africana, 1988 ; PRÖTTEL Ph.M., Mediterrane Feinkeramikimporte, 1996, p. 8-23.

77. Mackinsex M., El Mabrine, 1993, 1993, p. 167-170 ; Rlynolis P.. Trade, 1995, p. 6.

78. Caranidini A. et al., Atlante I, 1981, p. 19.

79. PRÖTTEI. Ph.M., Mediterrane Feinkeramikimporte, 1996, p. 25.

80. Sites $n^{\prime \prime} 4,6,13,16,21,29,32,41,44,47,58,61,65,70,96,118$, 119, 123, 127bis, 134, 152, 162, 163, 164, 165, 167, 169, 171, 175, 176, 181, 186, 188, 194, 196, 200, 202, 210

81. Sites $n^{\circ 1} 16,119$ et 152 cas (sites nos 119 et 152), le matériel associé n'est pas antérieur au III $^{\mathrm{e}} \mathrm{s}$.

\section{Catalogue}

-25 : céramique sigillée africaine $A$, forme Hayes $3 \mathrm{~B}$; décor de feuilles d'eau à la barbotine ; pâte beige orangé, feuilletée, vacuolaire, petites incl. de quartz; vernis orange usé. (Inv. 016.07)

\section{- Sigillée africaine $A / D$ (fig. 5)}

La production A/D apparaît sur 11 sites $^{82}$ majoritairement groupés au sud de la Chebba ${ }^{83}$. Les formes les plus fréquentes sont les assiettes Hayes $31\left(\mathbf{n}^{\circ} \mathbf{2 6}\right)$, 32 et 33, mais il convient d'attribuer également à cette production un objet plus insolite $\left(\mathbf{n}^{\circ} \mathbf{2 7}\right)$, une assiette à bord découpé ( 4 ou 6 pans) qui trouve une comparaison à Sperlonga ${ }^{84}$.

\section{Catalogue}

- 26 : céramique sigillée africaine A/D, forme Hayes 31 ; pâte rouge brique, grossière, grosses incl. de quartz ; vernis orange vif, épais, brillant. (Inv. 068.01)

- 27 : céramique sigillée africaine $\mathrm{A} / \mathrm{D}$, forme inédite ; pâte orange, grossière, incl. de quartz; vernis orange vif, épais, brillant. (Inv. 088.03)

\section{- Sigillée africaine $C$ (fig. 5)}

Les 38 sites $^{85}$ où ont été trouvés des tessons de sigillée africaine $\mathrm{C}$ (produite par les ateliers du centre de la Tunisie) sont assez uniformément espacés du sud au nord du littoral ; on y remarque une fréquence particulière des formes Hayes 50 et 72 . La production tardive $\mathrm{C} 5$ (deuxième moitié du ve $\mathrm{s}$.) n'est bien représentée que sur les sites de la côte est, avec les formes

82. Sites n's $1,5,8,35,42,44,68,79,83,88,165$

83. L'un des principaux ateliers, actuellement reconnus, de cette production est à Henchir el Guellal Djilma à environ $180 \mathrm{~km}$ à l'ouest de La Chebba : PEacock D.P.S., BeN Lazreg N. et Bejaoli F., Roman Pottery Production, 1990, fig. 1 ; PröTTHI. Ph.M., Mediterrane Feinkeramikimporte, 1996, p. 20.

84. SAGuì L., Sperlonga, 1980, p. 500 et fig. 39a-b : classé dans la catégorie $\mathrm{D}$ mais l'auteur précise que " la vernice, brillante (...) copre all'esterno anche il fondo *; ne pourrait-il pas s'agir d'une production A/D ? Cf. Caranidivi A. et al., Atlante I, 1981, pl. XL, $n^{\circ} 5$. Comparaison moins convaincante : forme La Baume, Salomonson 1976, pl. 54, n² 2 $=$ Atlante I, pl. $\mathrm{CXXXV}, \mathrm{n}^{\circ} 4$.

85. Sites n" $4,5, \underline{13}, \underline{32}, \underline{37}, \underline{38}, 41,42, \underline{43}, \underline{47}, \underline{54}, \underline{55}, 59,68, \underline{70}, \underline{71}$, $\underline{72}, 80,82,83,117,119,125,126, \underline{127}, 128, \underline{130}, 134,136,145,146$, $152,161,162,165,183,204,205$. Les chiffres soulignés correspondent à la production $\mathrm{C} 5$. 

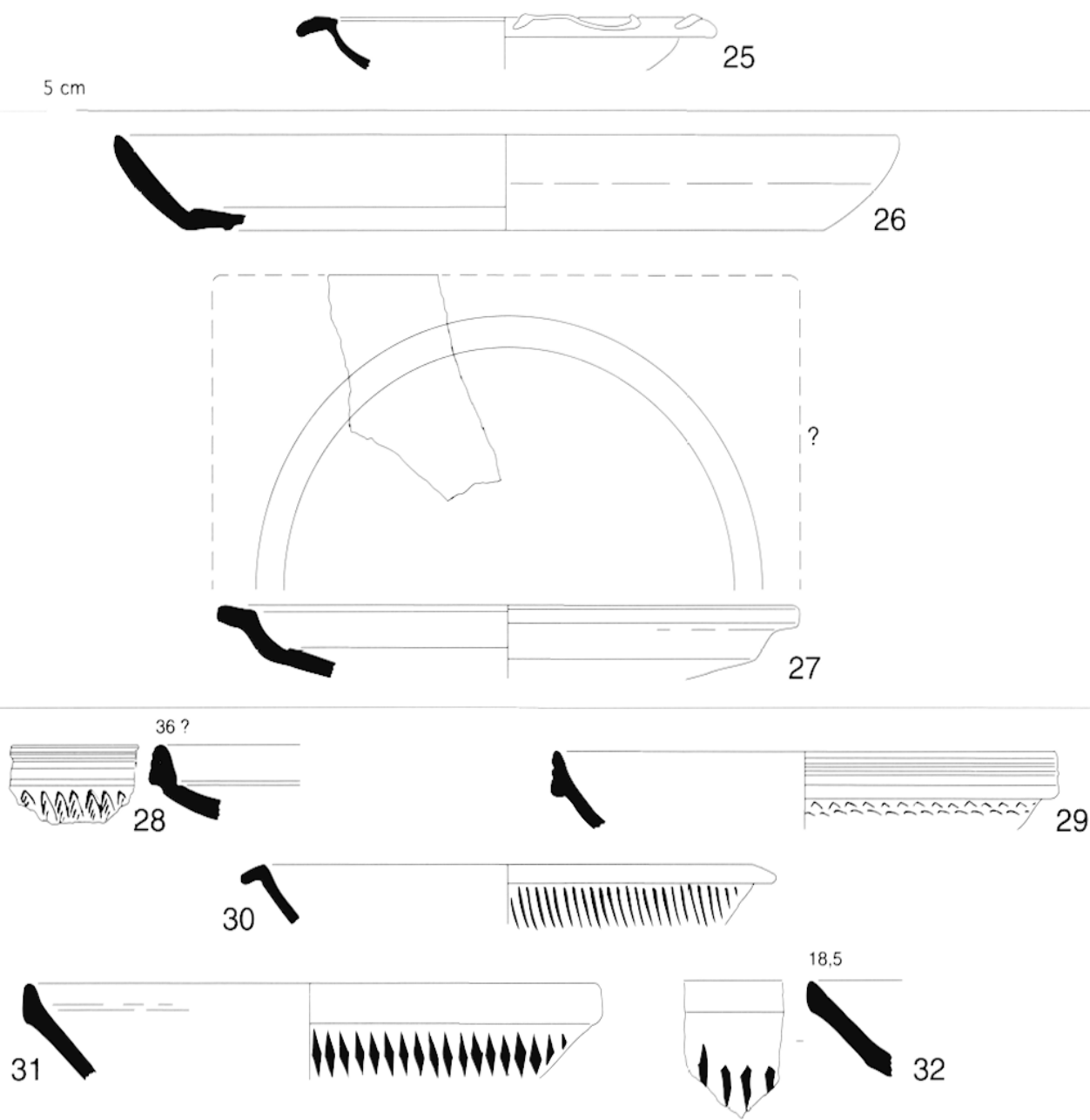

0
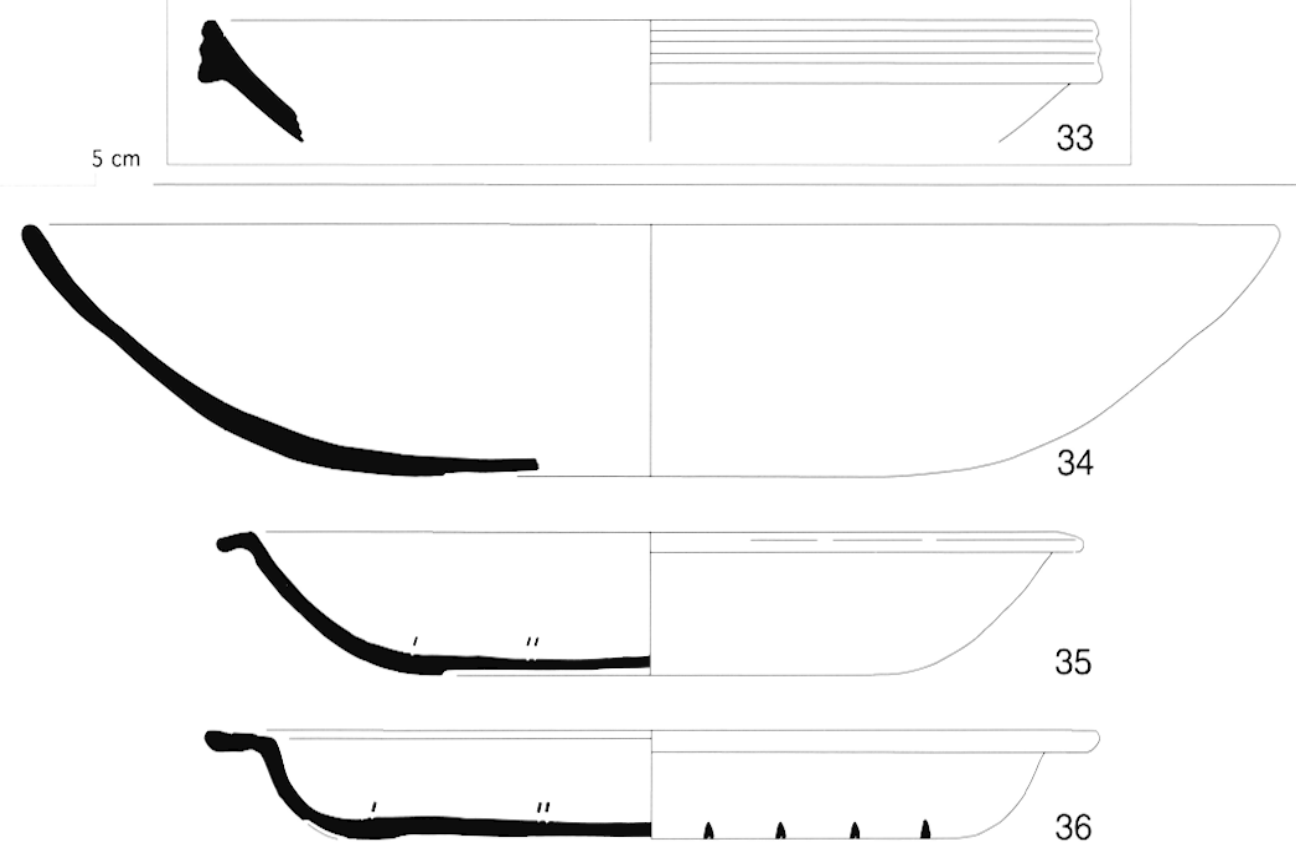

Fig. 5 : Sigillée africaine A ( $\left.{ }^{\circ} 25\right)$; sigillée africaine A/D ( $\left.n^{\circ s} 26-27\right)$; sigillée africaine $C 5$ et apparentée ( ${ }^{*}$ 28-32) ; sigillée africaine D (atelier de Sidi Khalifa) ( $\left.\mathrm{n}^{\circ} 33\right)$; sigillée africaine D ( $\left.\mathrm{n}^{\circ s} 34-36\right)$. (M. Bonifay) 
Hayes $82\left(\mathbf{n}^{\circ} \mathbf{2 8}\right), 84\left(\mathbf{n}^{\circ} \mathbf{2 9}\right), 85$, et une forme non classée $\left(\mathbf{n}^{\circ} \mathbf{3 0}\right)$ qu'il convient de distinguer de la forme Hayes 94 mais pour laquelle, toutefois, les références abondent ${ }^{86}$, y compris sur l'atelier de Sidi Marzouk Tounsi ${ }^{87}$. La forme Fulford $27^{88}$ (n 31 et 32), dans une qualité de pâte plus grossière, avec un vernis mat, granuleux au toucher, constitue une série parallèle à la forme Hayes 84 , également exportée ${ }^{89}$, mais dont la fabrication n'est jusqu'à présent attestée que sur des ateliers septentrionaux ${ }^{90}$. Enfin, il apparait que l'atelier de Sidi Khalif $a^{91}$ produisait également une forme apparentée à la forme Hayes $84^{92}$, probablement une forme Hayes 86 de grand diamètre, sans guillochis $\left(\mathbf{n}^{\circ} \mathbf{3 3}\right)$ : ici, la confusion avec les ateliers du centre de la Tunisie est facile ${ }^{y 3}$ car certaines productions de Sidi Khalifa ont une texture très fine ${ }^{94}$.

\section{Catalogue}

- 28 : céramique sigillée africaine $\mathrm{C} 5$, forme Hayes 82 ; décor de guillochis ; pâte orange, compacte ; vernis orange clair, satiné. (Inv. 070.04)

86. Mactar : Bourgeols A., Sanctuairc d'Hoter Miskar, 1982, fig. 4, $\mathrm{n}^{\text {os }}$ 178-179 ; Belo : BoljRGFols A. et MAYET Fr., Belo, 1991, pl. CIV, $\mathrm{n}^{\circ}$ 425-426 ; Marseille : GOURVEST J., Introduction, 1998 [1958], forme E3, fig. 24, nos 260-262 ; BonifaY M., Éléments d'évolution, 1983, fig. 22, nos 41-42; Brtev St., Contextes de l'Antiquité tardive, 1998, fig. $238, n^{\circ} 3$; Naples : SORICElli G., Terra Sigillata, 1994, fig. 64, $\mathrm{n}^{\mathrm{N}} 34-35$.

87. Placock D.P.S., Bin Lazreg N. et BrjaOUI F., Roman Pottery Production, 1990, fig. 7, $\mathrm{n}^{\circ} 14$.

88. Fllford M. et Pfacock D.P.S., Avenue Bourguiba, 1984, p. 57 et fig. 14 ; GANDOLFI D., Terra Sigillata Chiara D, 1981, p. 109, forme $60 \mathrm{C}$.

89. Par exemple, Tarragone : AQLiLlÉ X., Decoration espatulada, 1993, p. 139 et fig. $98, \mathrm{n}^{\circ \mathrm{s}} 86-87$; Marseille : BONIFAY M., Problèmes de datation, 1998, p. 363 ; Naples : Soricflu. G., Terra Sigillata, 1994, fig. $66, \mathrm{n}^{\circ} 55$; voir également ReYNOLDS P., Trade, 1995, fig. 12.

90. GHalla T., Boniray M. et Caplitil Cl., Sidi Zahruni, à paraître.

91. Visité en octobre 1997 en compagnie du responsable du site M. Samir Aounallah; qu'il soit ici remercié.

92. Voir les exemplaires de Tarragone : AQUILUE X., Las ceramicas finas, 1993, p. 133 et fig. $96, n^{\circ}$ 62-64.

93. Il en est probablement ainsi pour une bonne part des assiettes Hayes 84 retrouvées au cours de la prospection dans la vallée de Segermes (LI:ND J., Segermes Valley, 1995, p. 514-515: 47 exemplaires pour seulement 3 tessons de la forme 82 et aucun de la forme 85) : j'interpréterais volontiers l'exemplaire illustré fig. 11 comme une production de l'atelier de Sidi Khalifa.

94. Observation déjà faite par J.W. Hayes à propos de la forme 88 HAYFs J.W.. Late Roman Pottery. 1972, p. 136 et 292.
- 29 : céramique sigillée africaine $\mathrm{C} 5$, forme Hayes 84 (variante de petites dimensions); décor de guillochis ; pâte rouge brique, compacte, sans incl. ; vernis orange sombre, satiné. (Inv. 054.07)

- 30 : céramique sigillée africaine $\mathrm{C} 5$, forme Bourgeois 1982, fig. 4, nos 178-179 ; décor de guillochis ; pâte orange, compacte ; vernis orange clair, satiné. (Inv. 055.05)

- 31: céramique sigillée africaine de type indéterminé, forme apparentée à Fulford 27 ; décor de guillochis ; pâte rouge brique, grossière, incl. blanches et grises ; engobe orange, mat. (Inv, 078.09)

- 32 : céramique sigillée africaine de type indéterminé, forme apparentée à Fulford 27 ; décor de guillochis. (Inv. 055.07)

- 33 : céramique sigillée africaine $D$, forme Hayes 86 variante (Lamboglia 60 ) ; pâte orange clair, assez grossière, petites incl. de quartz ; vernis orange clair, lisse, satiné. (Atelier de Sidi Khalifa, exemplaire de comparaison)

\section{- Sigillée africaine $D$ (fig. 5 et 6)}

La sigillée africaine $D$ est la céramique la mieux diffusée sur le littoral tunisien : on la trouve sur 93 des 207 sites où des ramassages ont eu lieu. Il faut toutefois remarquer que cette catégorie réunit en fait des productions très diverses ; on en voudra pour preuve la complexité de sa classification interne ${ }^{95}$. Appartiennent à la première phase de la production (IV $\mathrm{s}$.) des formes Hayes $50 \mathrm{~B}\left(\mathbf{n}^{\circ} \mathbf{3 4}\right), 58\left(\mathbf{n}^{\circ} \mathbf{3 5}\right)$ et $59 \mathrm{~A}$ avec un exemplaire de petite taille non décoré96 $\left(\mathbf{n}^{\circ} 36\right)$. La forme Hayes 59 est essentiellement diffusée dans la partie nord du littoral ${ }^{97}$.

Caractéristique du ve s., la forme Hayes 61 B est présente sur 14 sites, du sud au nord du littoral98. La grande diffusion de cette forme ainsi que son manque d'homogénéité morphologique ${ }^{99}$ (nos 37 à 40) interdisent d'en attribuer la production à un seul atelier, même si l'un d'eux, Sidi Khalifa, paraît de ce point de vue très important $\left(\mathbf{n}^{\circ} \mathbf{4 1}\right)$. Le bord de plat Hayes 76 (?) à décor de godrons $\left(\mathbf{n}^{\circ} \mathbf{4 2}\right)$, attesté sur l'atelier de

95. Ibid., p. 291-292; CARANisinl A. et al., Atlante I, 1981, p. 78-79. Cette classification rend compte à la fois de différences d'ateliers et de chronologie (quatre siècles d'existence !).

96. HAYES J.W., Late Roman Pottery, 1972, p. 96 : exemplaires de D. $27 \mathrm{~cm}$, sans décor : $\mathrm{n}^{\text {\% }} 6$ ? et 16 .

97. Sites nos 1, 16, 128, 186, 200, 201, 203, 204, 207.

98. Sites $\mathrm{n}^{\circ} 1,19,21,54,79,98,138,139,146,147,164,187,198$, 200.

99. BONIFAY M.. Problèmes de datation, 1998. 
Djilma ${ }^{100}$, devrait plutôt être rattaché à la sigillée africaine $\mathrm{C}$. On ne sait pas où étaient produites les formes Hayes 87 : leur diffusion sur la côte est semble exclure une officine de la vallée de la Mejerda, ce que l'on savait déjà ${ }^{101}$, mais peut-être serait-ce trop schématiser que d'attribuer leur origine au seul atelier de Sidi Khalifa ${ }^{102}$. De ce point de vue, la distinction faite sur le site de Saint-Blaise (France) de deux variantes dans la forme Hayes 87 A me paraît intéressante ${ }^{103}$ : le "type 1 " (= Hayes $\left.87 \mathrm{~A}, \mathrm{n}^{\circ} 2\right)$ est caractérisé par " une lèvre arrondie et courte et un pied atrophié et bas " ainsi que par une décoration de style $\mathrm{E}(\mathrm{i})^{104}$; le "type 2 " (= Hayes $87 \mathrm{~A}, \mathrm{n}^{\circ}$ 1) a " une lèvre triangulaire et haute, un pied annulaire épais et triangulaire " et porte une décoration lustrée de bandes concentriques et de motifs géométriques, floraux ou chrétiens ${ }^{105}$. Il n'est pas impossible que l'atelier d'Oudhna ait produit une partie au moins des exemplaires du " type 1 "106; en revanche on ne connaît pas l'origine des produits à décor lustré. La diffusion de cette forme sur le littoral montre deux groupes : le premier concerne le Cap Bon et le golfe d'Hammamet ${ }^{107}$, le second le golfe de Gabès au sud du Rass Jezira ${ }^{108}$. On peut se demander s'il n'y aurait pas une production des formes Hayes 87 dans cette partie de la Tunisie. En effet, le décor lustré $\mathbf{n}^{\circ} \mathbf{5 2}$ a été trouvé sur le site $\mathrm{n}^{\circ} 70$, tandis que le bord Hayes $87 \mathrm{~B} \mathbf{n}^{\circ} \mathbf{4 4}$, trouvé

100. Peacock D.P.S., Ben LazReg N. et Bejaoli F., Roman Pottery Production, 1990 , fig. $13, n^{\circ} 19$. Un exemplaire tout à fait comparable à Marseille : BONIfaY M., Éléments d'évolution, 1983, fig. 26, nº 142. 101. MACKENSiN M., El Mahrine, 1993.

102. De fait, je n'ai pas remarqué une fréquence particulière de la forme Hayes 87 (un seul tesson, variante B) dans la zone de l'atelier de Sidi Khalifa que j'ai visitée. Les trois formes les plus répétitives sont Hayes $61 \mathrm{~B}, 88$ et 103. De même, si l'on considère que les prospections de la vallée de Segermes constituent un bon observatoire des productions de l'atelier de Sidi Khalifa, en raison de la proximité de celui-ci, on observe, au sein des sigillées africaines, une sur-représentation des formes Hayes 61B (81 ex.), 88 (71 ex. dont 2 ex. * 87/88 *) et 103 (217 ex.) alors que les différentes variantes de la forme 87 ne dépassent pas 25 exemplaires. Même faciès sur les sites du littoral de la sebkha Sidi Khalifa que nous avons explorés (sites n $\mathrm{n}^{\circ \mathrm{s}} 127$ à 129).

103. CaVAllitis-LLopis M. et Valialiri L., Céramiques fines importées, 1994, p. 96 et fig. 49. Voir également Gourvest J., Introduction, 1998 [1958], fig. $17, \mathrm{n}^{\circ} 175:$ type 1 et $174:$ type 2.

104. Palmette type Hayes 118 à Saint-Blaise, croix et oiseau type Hayes 309 sur l'exemplaire de Corinthe.

105. "Burnished patterns ", cf. AQuilut́ X., Decoracion espatulada, 1992. Les décors lustrés sont toujours sur les variantes A et C.

106. Attribution déjà suggérée dans Caranidnsi A. et al, Atlante I, 1981 , p. 94, en fonction du style décoratif.

107. Sites $n^{\text {(s) }} 125,129,131,133,135,139,140,162,164,165$.

108. Sites n's $16,19,21,37,42,54,55,70,71,78,84$. à Onga, pourrait être un surcuit (?) ; enfin le tesson $\mathbf{n}^{\circ} \mathbf{4 3}$ (Jerba) présente un aspect particulièrement fruste ${ }^{109}$. Les incertitudes s'atténuent quelque peu avec la forme Hayes 88 : particulièrement abondante sur le rivage de la sebkha Sidi Khalifa' ${ }^{110}$, elle est sans doute ${ }^{111}$, en grande partie, originaire de l'atelier du même nom ${ }^{112}$. La forme Hayes 99, probablement en très grande partie produite par l'atelier d'Oudhna ${ }^{113}$, a été rencontrée sur 17 sites avec une forte concentration dans le Cap Bon ${ }^{114}$. Enfin, on doit admettre la pluralité des sources de la forme Hayes 103 ( $\left.\mathbf{n}^{\circ} \mathbf{4 8}\right)$ diffusée sur tout le littoral115.

Le matériel des prospections du littoral tunisien montre une large diffusion des productions de la fin du $\mathrm{VI}^{\mathrm{e}}$ et du $\mathrm{VII}^{\mathrm{e}} \mathrm{s}$. Le plat Hayes $90 \mathrm{~B}\left(\mathbf{n}^{\circ} \mathbf{4 6}\right)$, présent sur 12 sites avec une densité particulière entre Sfax et Salakta ${ }^{116}$, pourrait être originaire, principalement ${ }^{117}$, d'ateliers du centre de la Tunisie ${ }^{118}$. De même, si les formes Hayes $91 \mathrm{D}\left(\mathbf{n}^{\circ} \mathbf{4 7}\right)$ et $105\left(\mathbf{n}^{\circ} \mathbf{5 0}\right)$ se révèlent largement réparties sur tout le littoral ${ }^{119}$, on remarque une certaine concentration de cette dernière forme en Byzacène ${ }^{120}$, peut-être révélatrice d'un atelier impor-

109. Attesté également sur le site de Rougga-Bararus au sud d'El Jem.

110. Sites nos 37, 70, 78, 106, 121, 125, 127, $128, \underline{129}, 131,133,164$. Les sites où la forme Hayes 88 est particulièrement abondante sont soulignés.

111. Un consensus semble s'établir sur ce point : CaRANDINI A. $e t$ al., Atlante I, 1981, p. 93. Voir cependant ReYNol.Ds P., Trade, 1995, p. 6.

112. Sur l'atelier de Sidi Khalifa on marche littéralement sur les tessons de Hayes 88, comme à Oudhna sur ceux de Hayes $99 .$.

113. BARRAJd D. et al., Industrie céramique, 1998. Voir cependant MACKLNSEN M., El Mabrine, 1993, forme El Mahrine 29.

114. Sites nos 49, 54, 70, 105, 110, 120, 137, 157, 159, 161, 165, 171 , 175, 200, 201, 203bis, 207.

115. Sites nos $29,42,47,70,107, \underline{127}, \underline{128}, 129,131,137,173,208$. Les sites où la forme Hayes 103 est particulièrement abondante sont soulignés.

116. Sites nos 55, 70, 72, 77, 78, 80, 84, 94, 137, 165, 198, 206.

117. Production (marginale ?) d'une forme comparable sur l'atelier d'El Mahrine (forme 45) : MACKlisis M., El Mabrine, 1993.

118. Cette forme, assez rare à Carthage (FLLFord M. et PFACOCK D.P.S., Avenue Bourguiba, 1984, p. 63 ; voir cependant RILEY J., Cistern, 1981 , p. 104 et fig. 5, nos 4-5) est particulièrement abondante à Rougga-Bararus. Les exemplaires importés dans le Midi de la Gaule ont un aspect comparable à ceux de Rougga.

119. Forme Hayes 91D : sites $n^{\alpha *} 21,50,54,70,78,137,198$. Forme Hayes 105 : sites n'시 $16,21, \underline{54}, 61,70,71,72,73,76,77,78,85,105$, $107, \underline{110}, 121,130, \underline{137}, 148,161,167,175,187,200,201$. Les sites où la forme Hayes 105 est particulièrement abondante sont soulignés.

120. Forme très fréquente à Lamta-Leptiminus : DORF: J., First report, 1992, p. 120 et 154. 

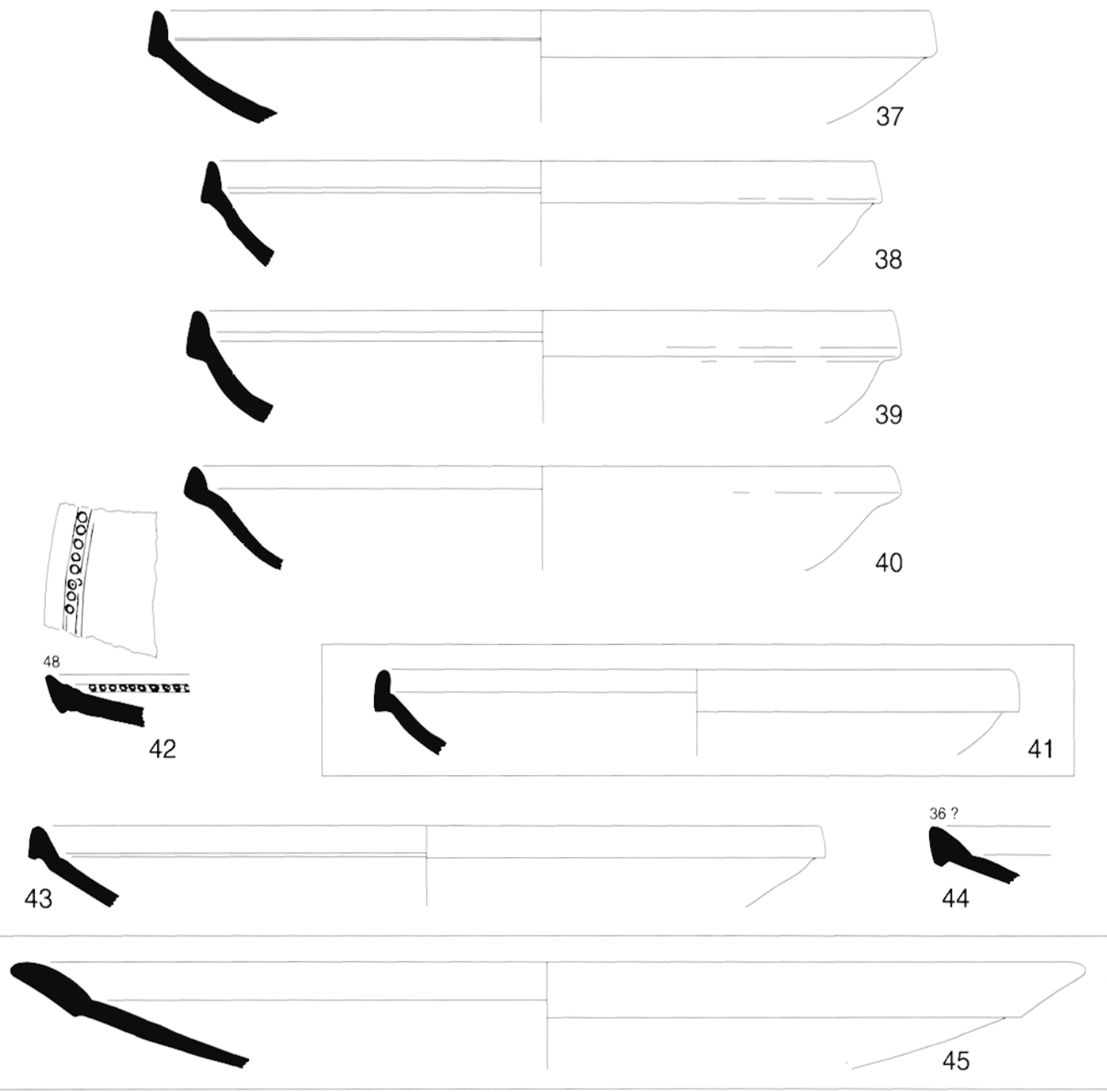

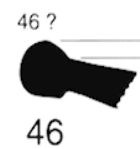

46
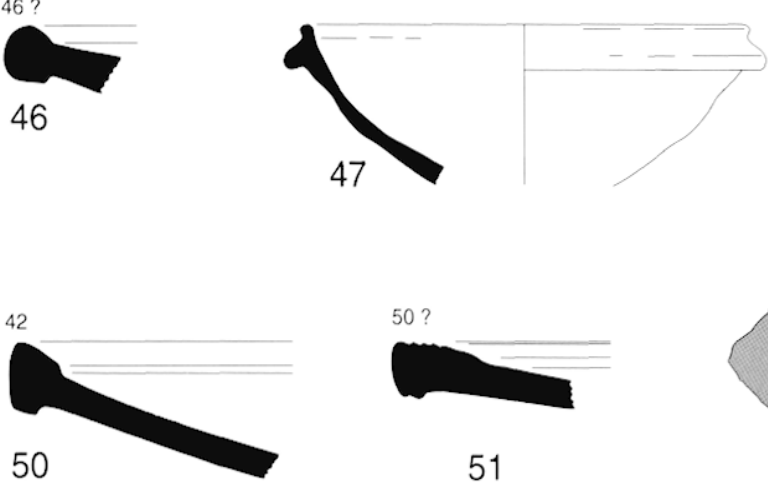

51
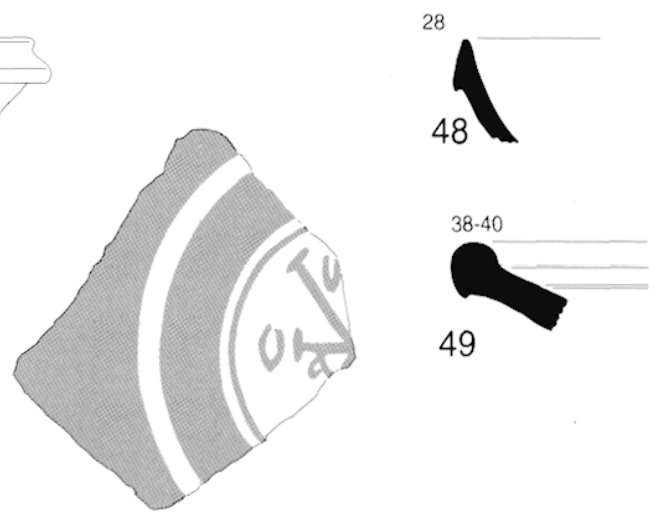

49

52

Fig. 6 : Sigillée africaine D (nos 41 et 45 : atelier de Sidi Khalifa). (M. Bonifay) 
tant dans cette région ${ }^{121}$. Enfin, la forme Hayes $104 \mathrm{C}$ $\left(\mathbf{n}^{\circ}\right.$ 49) et une variante tardive des formes $105 / 106$ (Atlante XLI, 3-4) ( ${ }^{\circ}$ 51) ne sont pas attestées de manière suffisamment fréquente pour que leur répartition soit significative ${ }^{122}$.

\section{Catalogue}

- 34 : céramique sigillée africaine $\mathrm{D}$, forme Hayes $50 \mathrm{~B}$. (Inv. 099.10)

- 35 : céramique sigillée africaine $\mathrm{D}$, forme Hayes 58 ; vase complet, non décoré. (Inv. 128.03)

- 36 : céramique sigillée africaine D, forme Hayes 59 A ; vase complet, non décoré. (Inv. 128.02)

- 37 : céramique sigillée africaine $\mathrm{D}$, forme Hayes $61 \mathrm{~B}$; pâte rouge brique, grossière, grosses incl. de quartz ; vernis orange luisant, irrégulier. (Inv. 019.01)

- 38 : céramique sigillée africaine $\mathrm{D}$, forme Hayes $61 \mathrm{~B}$; pâte rouge brique, grossière, nombreuses petites incl. translucides ; vernis orange. (Inv. 001.01)

- 39 : céramique sigillée africaine $\mathrm{D}$, forme Hayes $61 \mathrm{~B}$ variante. (Inv. 122.01)

- 40 : céramique sigillée africaine $\mathrm{D}$, forme Hayes $61 \mathrm{~B}$ variante. (Inv. 054.10)

- 41 : céramique sigillée africaine $\mathrm{D}$, forme Hayes $61 \mathrm{~B}$; pâte rouge brique, assez grossière, nombreuses petites incl. de quartz ; vernis orange, lisse, plutôt mat. (Atelier de Sidi Khalifa, exemplaire de comparaison)

- 42 : céramique sigillée africaine D (ou C5 ?), forme Hayes 76 variante (?) ; décor de faux godrons imprimés ; pâte orange, compacte ; vernis orange clair, satiné. (Inv. 071.01) - 43 : céramique sigillée africaine D (?), forme Hayes $87 \mathrm{~A}$ (?) ; pâte rouge brique, grossière, nombreuses grosses incl. de quartz ; vernis orange, irrégulier, bord ext. jaune (objet cuit hors casette ?). (Inv. 019.02)

- 44 : céramique sigillée africaine $D$, forme Hayes 87 B ; pâte brune, assez grossière, petites incl. translucides ;

121. Ces deux formes ne semblent pas produites sur l'atelier d'El Mahrine mais elles ne sont pas rares à Carthage.

122. La forme Hayes 104C, attestée notamment à Carthage (HAYEs J.W., Pottery report, 1978, p. 50 et fig. 12, nos 1-2 ; RILEY J., Cistern, 1981 , p. 104 et fig. 6, nos 28-29) et à Sidi Jdidi (BEN ABED A., BONIFAY M. et FiXot M., Note préliminaire, 1997, p. 16 et fig. 3, n 19), est présente sur les sites nos 50 et 88 . La forme $105 / 106$ variante, présente sur l'atelier d'El Mahrine (MACKENSEN M., El Mabrine, 1993, forme 43), à Carthage (HAYES J.W., Pottery report, 1978, p. 50 et fig. 12, n 4 ), à Sidi Jdidi (BEN ABEI) A., BONIIAY M. et FIXOT M., Note préliminaire, 1997, p. 22 et fig. 9, n 46), à Rougga (GléRY R. † et BonifaY M., Céramiques antiques, à paraître), a été trouvée en un seul exemplaire (site $\left.\mathrm{n}^{\circ} 101\right)$. vernis brun (surcuit ?), coulures à l'ext. montrant que la rotation du tour se faisait dans le sens des aiguilles d'une montre. (Inv. 054.08)

- 45 : céramique sigillée africaine $D$, forme Hayes 88 ; pâte orange, assez grossière ; vernis orange clair, lisse, satiné. (Atelier de Sidi Khalifa, exemplaire de comparaison)

- 46 : céramique sigillée africaine $\mathrm{D}$, forme Hayes $90 \mathrm{~B}$; pâte orange, grossière ; vernis orange clair, mat. (Inv. 070.02)

- 47 : céramique sigillée africaine D, forme Hayes $91 \mathrm{D}$. (Inv. 050.02)

- 48 : céramique sigillée africaine D, forme Hayes 103 B (?) ; pâte rouge brique, grossière ; vernis orange vif, brillant. (Inv. 029.01)

- 49 : céramique sigillée africaine D, forme Hayes $104 \mathrm{C}$. (Inv. 050.01)

- 50 : céramique sigillée africaine $D$, forme Hayes 105 ; pâte rouge brique, grossière ; vernis orange clair, mat. (Inv. 071.04)

- 51 : céramique sigillée africaine $\mathrm{D}$, forme Atlante $\mathrm{XLI}, 3-$ 4. (Inv. 101.01)

- 52 : céramique sigillée africaine $\mathrm{D}$, forme Hayes $87 \mathrm{~A}$ (?) ; décor lustré : chrisme ; pâte rouge brique, grossière ; vernis orange, brillant. (Inv. 070.01)

\section{- Sigillée africaine $E$ et autres sigillées africaines (fig. 7)}

La sigillée africaine E, production du Sud tunisien entre la fin du Ive $s$. et le milieu du ve $s .{ }^{123}$, est représentée au sein de notre matériel par un ensemble de formes : Hayes $66\left(\mathbf{n}^{\circ} \mathbf{5 3}\right), 68\left(\mathbf{n}^{\mathbf{o s}} \mathbf{5 4}\right.$ à $\mathbf{5 8} ; \mathbf{5 9}$ à $\mathbf{6 2}$ ?), 70 et $92\left(\mathbf{n}^{\circ} \mathbf{6 3}\right)$, dont la diffusion est concentrée dans la partie sud du littoral ; on notera toutefois la présence de quelques exemplaires sur la côte nord $^{124}$. Les variations importantes dans le profil des plats Hayes 68 laissent envisager une multiplicité d'ateliers. Ceux qui ont été reconnus dans la région de Sbeitla, à Henchir es Srira ${ }^{125}$ et Henchir el Guellal Djilma ${ }^{126}$, et d'autres plus au Sud-Ouest, à Sidi

123. Carandini A. et al., Atlante I, 1981, p. 119.

124. Forme Hayes 66 : sites $n^{\circ} 37$ et 198 . Forme Hayes 68 : sites $\mathrm{n}^{\mathrm{os}} 1,5,8, \underline{16}, 27,31,32,33,35,37,38,39,41,42,43,54,55,70,73$, 76, 99 (?), 146, 147, 157, 200. Forme Hayes 70 : sites $\mathrm{n}^{\mathrm{os}} 16,47,55$. Forme Hayes 92 : un seul exemplaire (site $n^{\circ} 37$ ). Les sites où la forme Hayes 68 est particulièrement abondante sont soulignés.

125. STERN M., Henchir es Srira et Sidi Aïch, 1968 ; PEACOCK D.P.S., Brin LazReg N. et BejaOu F., Roman Pottery Production, 1990, p. 74-76. 126. Ibid., p. 76-79. 

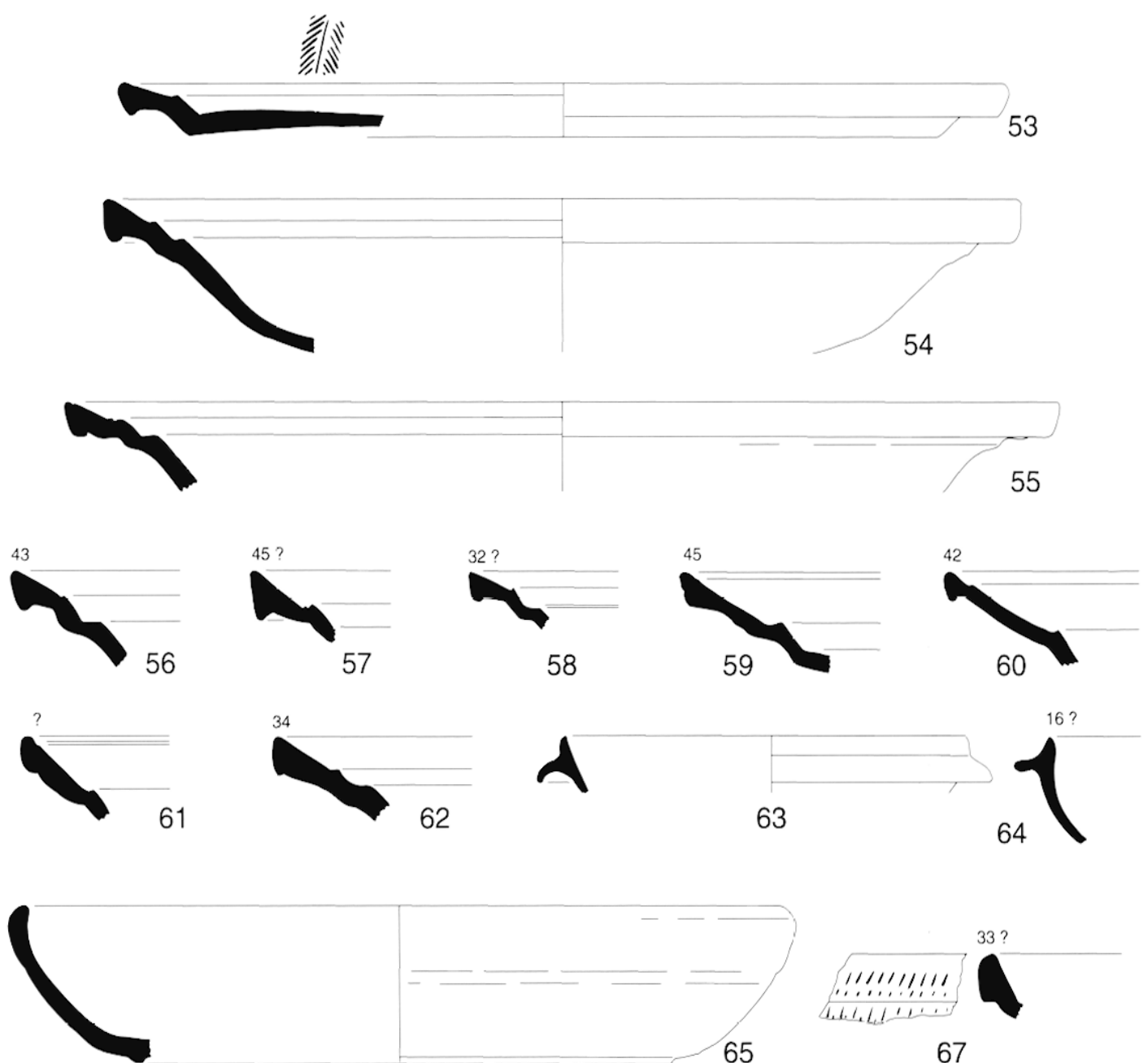

65

67
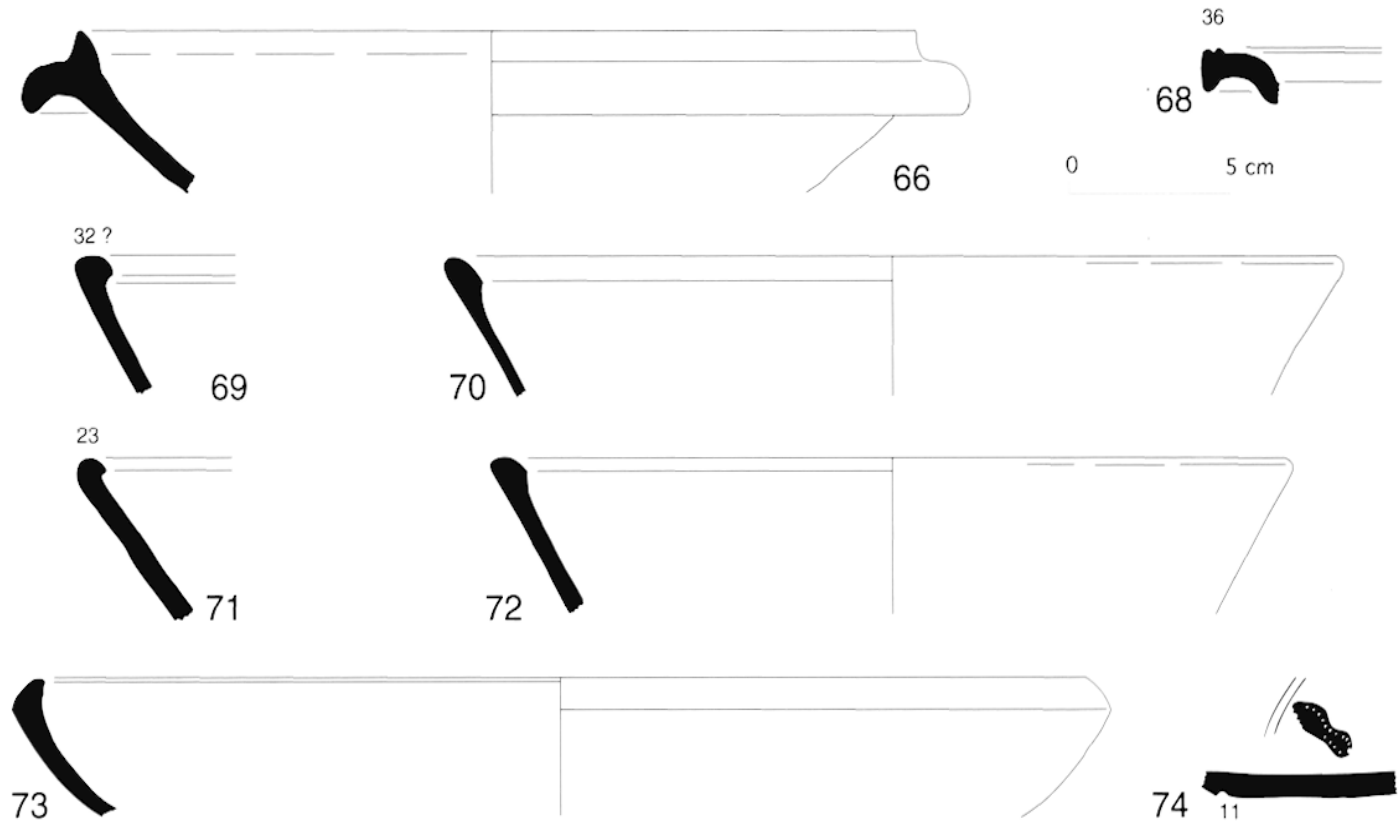

Fig. 7 : Sigillée africaine $E\left(n^{\circ 5} 53-64\right)$; autres sigillées africaines ( $\left.{ }^{\circ \infty} 65-74\right)$. (M. Bonifay) 
Aïch ${ }^{127}$ et Thelepte ${ }^{128}$, ont, semble-t-il, produit certaines formes apparentées à la sigillée africaine E. Le profil particulier des plats $n^{\text {os }} 59$ à 61 se rapproche de certains produits de Henchir el Guellal Djilma ${ }^{129}$, tandis que le tesson $n^{\circ} 62$ évoque ceux de I Ienchir es Srira ${ }^{130}$. Peut-être reste-t-il à découvrir un atelier important qui aurait pu manufacturer les formes classiques de la sigillée E (nos 54 à 58). Notre prospection n'a pas apporté de preuve formelle de la production de cette sigillée sur le littoral, malgré quelques présomptions à Oued el Akarit (voir infra, $\mathbf{n}^{\circ}$ 219).

C'est sans doute encore aux ateliers du Sud-Ouest tunisien qu'il convient d'attribuer les vases $\mathrm{n}^{\circ} \mathbf{6 5}$ (forme Stern Id), $\mathbf{n}^{\circ} \mathbf{6 6}$ (Stern XXIX), $n^{\circ} 67$ (Stern XXXV), $\mathbf{n}^{\circ} \mathbf{6 8}$ (Stern X). La forme Stern IV (= forme Hayes 1972, fig. 58 a.) (nos 69 à 72), très fréquente dans la région de Kasserine ${ }^{131}$, est également assez répétitive sur plusieurs sites du golfe de Gabès ${ }^{132}$. Enfin, on ne sait si le plat $\mathbf{n}^{\circ} \mathbf{7 3}$ doit être rattaché à ces mêmes ateliers ( = forme Hayes 1972, fig. 58 b) ou bien à la Tripolitanian Red Slip Ware (forme 3) ${ }^{133}$. Ces ateliers ont peut-être eu une diffusion plus grande qu'on ne le pense, en irriguant une partie de la côte tripolitaine septentrionale et certainement aussi de nombreux sites de l'actuelle Algérie intérieure ${ }^{134}$. Notuns enfin, de manière liminaire, que les productions plus spécifiquement algériennes ${ }^{135}$ ne paraissent pas très bien diffusées sur le littoral tunisien ${ }^{136}$.

127. STlikN M., Henchir es Srira et Sidi Aïch, 1968.

128. Sur l'atelier de Thelepte et sur celui de Sidi Aïch, voir les travaux en cours de M. Mongi Nasr : NASR M., Recherches sur la céramique rouge orange dans la région de Gafsa à l'époque romaine : l'atelier de Sidi Aïch, Certificat d'Aptitude à la Recherche, Université de Tunis, 1992 (dactylographié), 130 p., 48 pl. ; NASR M., Recherches sur la sigillée claire africaine de la Byzacène du Sud-ouest : les dépotoirs de Thelepte, Diplôme d'Études Approfondies, Université de Provence, 1995 (dactylographié), 102 p., 16 pl.

129. Peacock D.P.S., Ben Lazreg N. et Bejaoli F., Roman Pottery Production, 1990, fig. 13, n ${ }^{\circ}$ 21-23.

130. Forme Stern VII, qui possède une paroi plus épaisse et un profil moins anguleux que la forme Hayes 68 .

131. Nitru L., Red Slipped wares, 1987, p. 181 et fig. 4, nos 35-41.

132. Diffusion de l'ensemble de ces formes : sites nos $10,12,30,31$, $37,38,41,47$.

133. Voir également Netrt: L., Red Slipped wares, 1987, p. 181.

134. FívriER P.-A., Remarques préliminaires, 1963, fig. 7 et 8.

135. FÉVrier P.-A., Sétif, 1965, fig. 32. à vernis métallescent.

136. Absentes du matériel récolté sur le littoral. Quelques exemplaires à Chemtou (?) : Vicias M., Céramique du camp, 1994, p. 151152, en particulier fig. 159, n⿳亠口冋 103-107.

\section{Catalogue}

- 53 : céramique sigillée africaine E, forme Hayes 66. (Inv. 037.14)

- 54 : céramique sigillée africaine $\mathrm{E}$, forme Hayes 68 . (Inv. 037.13)

- 55 : céramique sigillée africaine E, forme Hayes 68 ; pâte rouge brique, compacte; vernis rouge carmin, mat. (Inv. 035.02)

- 56 : céramique sigillée africaine $\mathrm{E}$, forme Hayes 68. (Inv. 099.06)

- 57 : céramique sigillée africaine E, forme Hayes 68 ; pâte beige orangé, compacte ; vernis rouge carmin, satiné, écaillé. (Inv. 037.01)

- 58 : céramique sigillée africaine $\mathrm{E}$, forme Hayes 68 ; pâte orange clair, compacte ; vernis rouge carmin, lisse, satiné. (Inv. 037.11)

- 59: céramique sigillée africaine $\mathrm{E}$, forme Hayes 68 variante. (Inv. 099.08)

- 60 : céramique sigillée africaine E, forme Hayes 68 variante. (Inv. 099.09)

- 61 : céramique sigillée africaine $\mathrm{E}$, forme Hayes 68 variante. (Inv. 099.07)

- 62 : céramique sigillée africaine $\mathrm{E}$, forme Hayes 68 variante ; pâte rouge brique, compacte, vacuolaire ; vernis rouge rosé, écaillé. (Inv. 033.03)

- 63 : céramique sigillée africaine $\mathrm{E}$, forme Hayes 92 ; pâte beige orangé, compacte ; vernis rouge carmin, satiné. (Inv. 037.02)

- 64 : céramique sigillée africaine E (?), forme Hayes 92 variante ; pâte rouge brique, assez grossière ; vernis orange clair, mat. (Inv. 070.03)

- 65 : céramique sigillée africaine indéterminée (sud-ouest de la Tunisie ?), forme Stern I d ; pâte rouge brique, assez grossière ; vernis orange, épais, brillant int. et ext. (Inv. 083.01) - 66 : céramique sigillée africaine indéterminée (sud-ouest de la Tunisie ?), forme apparentée à Stern XXIX ; pâte rouge brique, assez grossière ; vernis orange, épais, brillant. (Inv. 031.01)

- 67 : céramique sigillée africaine indéterminée (sud-ouest de la Tunisie, atelier de Sidi Aïch ?), forme Stern XXXV; pâte beige orangé, assez grossière mais compacte ; vernis rouge carmin, écaillé. (Inv. 054.06)

- 68 : céramique sigillée africaine indéterminée (sud-ouest de la Tunisie, atelier de Sidi Aïch ?), forme Stern X ; pâte orange, assez grossière mais compacte ; vernis rouge orangé, écaillé. (Inv. 010.01)

- 69 : céramique sigillée africaine indéterminée (sud-ouest de la Tunisie ?), forme Stern IV (Hayes 1972, fig. 58 b); 


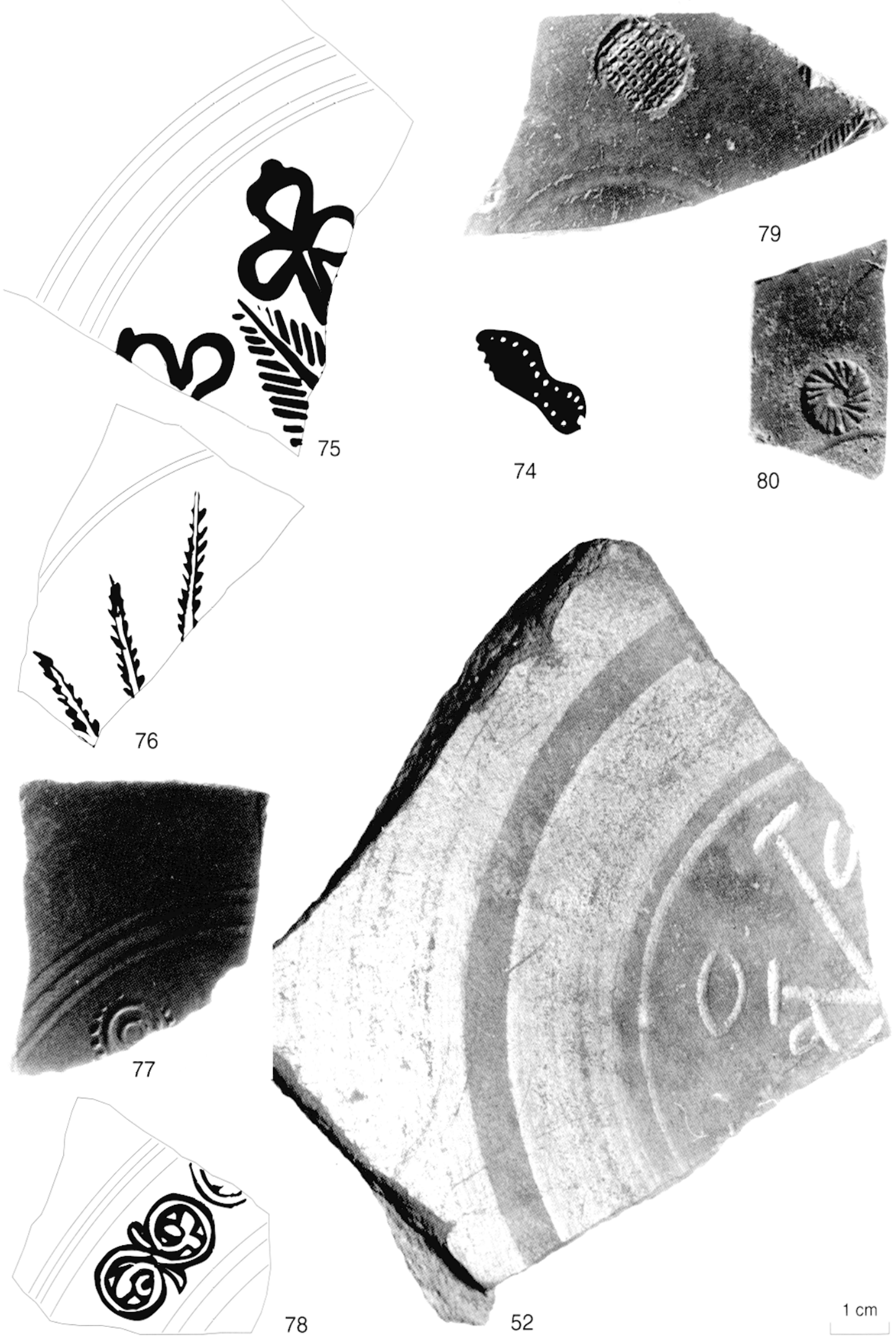

Fig. 8 : Décors sur sigillées africaines. (M. Bonifay ; photographies Chr. Durand) 
pâte rouge brique, grossière ; vernis orange vif, granuleux. (Inv. 037.12)

- 70 : céramique sigillée africaine indéterminée (sud-ouest de la Tunisie ?), forme Stern IV (Hayes 1972, fig. 58 b) ; pâte rouge brique, grossière ; vernis orange, mat. (Inv. 041.04)

- 71 : céramique sigillée africaine indéterminée (sud-ouest de la Tunisie ?), forme Stern IV (Hayes 1972, fig. 58 b). (Inv. 037.17)

- 72 : céramique sigillée africaine indéterminée (sud-ouest de la Tunisie?), forme Stern IV (Hayes 1972, fig. 58 b) ; pâte rouge brique, grossière ; vernis orange, brillant. (Inv. 041.03)

-73 : céramique sigillée africaine indéterminée (sud-ouest de la Tunisie ?), forme Hayes 1972, fig. 58 a, ou ${ }^{137}$ Tripolitanian Red Slip ware (?), forme Hayes 3 ; pâte beige orangé, grossière, assez compacte ; engobe orange, mat, lisse. (Inv. 012.01)

- 74 : céramique sigillée africaine indéterminée (sud-ouest de la Tunisie ?) ; décor imprimé (semelle de chaussure cloutée ?) : cf. Hayes 1972, fig. 59 c. (Inv. 037.18)

\section{- Décors sur sigillées africaines (fig. 8)}

Assez peu nombreux, ils nous renseignent cependant sur la diffusion des différents ateliers. Celui d'El Mahrine ( $\left.\mathbf{n}^{\circ} \mathbf{7 5}\right)$ est attesté, de manière banale, sur un site de la côte nord. Le poinçon répété selon le style A (iii) sur le tesson $\mathbf{n}^{\circ} \mathbf{7 6}$ est comparable à ceux que $M$. Mackensen propose d'attribuer à l'atelier de Sidi Khalifa ${ }^{138}$; de fait, on remarque qu'il provient d'un site très proche de cette officine. Bien que peu original, le $\mathbf{n}^{\circ} \mathbf{7 7}$ évoque un ensemble de décors bien attesté au milieu du ve $s$. à Marseille ${ }^{139}$. Le décor floral de style A (iii)/D ( $\left.\mathbf{n}^{\circ} \mathbf{7 8}\right)$ trouvé sur un site de la côte nord appartient à un groupe d'origine indéterminée, reconnu à Carthage et caractéristique de la deuxième moitié du ve s. ${ }^{140}$. Les $\mathbf{n}^{\text {os }} \mathbf{7 9}$ et $\mathbf{8 0}$ renvoient aux

137. Difficultés rencontrées par J.R. Timby à Sabratha : TimbY J.R., Red Slipped wares, 1994, p. 84.

138. MaCKensex M., El Mabrine, 1993, p. 446 et fig. 122 ; voir également DENiatve J., Céramiques et lampes africaines, 1972, pl. V, C770C771: Sa.NTaMaria Cl., Dramont E, 1995, p. 92, fig. 80.

139. BONIFAY M., Éléments d'évolution, 1983, fig. $19, \mathrm{n}^{\circ} 16$; COl:LRME:\%/() (1) Fl., Vaisselle du sondage 10, 1998, fig. 107, $n^{\circ} 51$; TREGIIA J.-Chr., Vaisselle des sondages 11 et 12, 1998, fig. 148, $\mathrm{n}^{\circ \mathrm{m}}$ 13-14.

1.1). F( I.FCRI M. et PBACOCK D.P.S.. Avenue Bourguiba, 1984. p. 107 et fig. 29 (seconde moitié du ve s.) ; voir également MACKFNSEN M., $E l$ Mabrine. 1993. p. 452-453 et note 114. À Marseille : Bovilay M., CARri: M.-B., Rigolr Y., Fouilles à Marseille, 1998, p. 365. productions des ateliers du Sud tunisien, avec des poinçons reconnus sur l'atelier de Sidi Aïch ${ }^{141}$ ou d'autres ( $\left.\mathbf{n}^{\circ} \mathbf{7 4}\right)$ qui évoquent la Tripolitanian Red Slip ware $^{142}$; leur diffusion est strictement méridionale. Pour le décor lustré $\mathbf{n}^{\circ} \mathbf{5 2}$, voir supra.

\section{Catalogue}

- 75 : céramique sigillée africaine $D$ (atelier d'El Mahrine ?) ; décor imprimé de style El Mahrine I.2 : trèfles et palmette El Mahrine 157 et 4.2. (Inv. 201.01)

- 76 : céramique sigillée africaine $\mathrm{D}$; décor imprimé : palmettes en disposition radiale, style Hayes A (iii)? (Inv. 128.01)

- 77 : céramique sigillée africaine D ; décor imprimé de style Hayes A (iii) (?) : rouelle ; pâte orange, granuleuse ; vernis orange clair, mat, granuleux au toucher. (Inv. 077.06) - 78 : céramique sigillée africaine $D$; décor imprimé de style A (iii) $/ \mathrm{D}$ : rinceaux proches de Fulford et Peacock 1984, fig. 29, n 97 . (Inv. 165.01)

- 79 : céramique sigillée africaine indéterminée (sud-ouest de la Tunisie ?); décor imprimé : cercle quadrillé ocelé (cf. Sidi Aïch S 8) et palmette à pédoncule (cf. Sidi Aïch S 2); pâte orange, granuleuse, compacte ; vernis rouge carmin, satiné (int.). (Inv. 037.04)

- 80 : céramique sigillée africaine indéterminée (sud-ouest de la Tunisie ?); décor imprimé : rouelle ; pâte orange, assez grossière mais compacte, incl. translucides ; vernis rouge carmin, écaillé. (Inv. 037.05)

\section{Les lampes (fig. 9)}

La lampe de la vallée du Pô ("Firmalampe ") signée $O C T A V I$, officine active de la fin du jer $s$. au milieu du $\mathrm{III}^{\mathrm{e}} \mathrm{s} .^{143}\left(\mathbf{n}^{\circ} \mathbf{8 1}\right)$ constitue une exception : en effet, les lampes recueillies lors de nos prospections sont quasiment toutes d'origine africaine. Pour les $\mathrm{II}^{\mathrm{e}}$ et $\mathrm{III}^{\mathrm{e}} \mathrm{s}$., les types Deneauve VII ( $\mathbf{n}^{\circ} 82$ ?) et VIII sont les plus fréquents, aux côtés du type $X\left(\mathbf{n}^{\circ} \mathbf{8 3}\right)$. Mais ce sont les lampes en sigillée africaine qui sont les mieux diffusées sur le littoral. Nous mettrons l'accent sur les sites de la Byzacène méridionale et de la Tripolitaine septentrionale qui livrent les types les plus anciens de cette production (sigillée africaine C) : Atlante I $\left(\mathbf{n}^{\circ} 84\right.$ ?) et IV (nos 85 et 86 ?). Cependant, les tessons les plus nombreux semblent se référer au type VI

141. STERN M., Henchir es Srira et Sidi Aïch, 1968, pl. I.

142. HaYYs J.W.. Late Roman Pottery. 1972. p. 303, fig. 59 c et 62 c. 143. Bt.CHI E., Lucerne del museo di Aquileia, 1975, p. 126-131. 
(nos 88 à 90 ?; nos 91 à 93), à situer probablement au IV $s$. et dont peu d'exemples ont été publiés à ce jour $^{144}$ : on remarquera tout particulièrement les lampes ornées d'un lion bondissant à gauche, décor qui semble relativement répandu ${ }^{145}$; une lampe comparable provient de l'atelier d'Oued el Akarit mais on ne peut affirmer qu'elle a été produite sur place (voir infra, $\mathbf{n}^{\circ} \mathbf{2 2 0}$ ). Toujours caractéristique des sites du centre et du sud du littoral, on note un fragment de lampe du type " de Navigius " $\left(\mathbf{n}^{\circ}\right.$ 96) et des exemplaires de lampes "chrétiennes " précoces de type Atlante VIII B ( $\left.\mathbf{n}^{\circ} \mathbf{9 5}\right)$; ces deux types de lampes semblent être produits, au moins en partie, sur l'atelier d'Henchir es Srira ${ }^{146}$. L'examen des lampes Atlante X découvertes dans la partie centro-méridionale du littoral confirme, s'il en était encore besoin, que le type Hayes II A, aux décors de petite taille, serrés les uns contre les autres et finement dessinés, est bien un type local (nos 97, 98, 99). Il n'est pas impossible que cette production soit encore vivace aux $\mathrm{VI}^{\mathrm{e}}-\mathrm{VII}^{\mathrm{e}} \mathrm{s}$. si l'on en juge par quelques exemplaires de lampes tardives (nos 100 et 256) : ces objets portent un décor caractéristique de cette période (grande croix gemmée, feuilles cordiformes sur le bandeau) mais paraissent conserver certaines particularités (décors de petite taille) propres aux ateliers du centre de la Tunisie. Le $\mathbf{n}^{\circ} \mathbf{1 0 1}$ constitue un des rares exemples en sigillée africaine des lampes tournées dites "vandales " 147 généralement fabriquées en céramique commune. Les lampes tripolitaines paraissent peu diffusées sur les sites du littoral que nous avons visités ; le site où a été trouvé le $\mathbf{n}^{\circ} \mathbf{1 0 2}$ est l'un des plus méridionaux. Enfin, il semble que les ateliers du centre de la Tunisie, tout comme ceux du Nord ${ }^{148}$, aient eu une production marginale de figurines en "céramique sigillée " $\left(\mathbf{n}^{\circ} \mathbf{1 0 3}\right)$.

144. Caranidini A. et al., Atlante I, 1981, p. 192.

145. Exemplaires comparables (agneau à la place du lion) au Musée de Sfax, provenant de La Skhirra : FFNDDRI M., Basiliques chrétiennes, 1961, pl. XXXVI, n 4 ; sur le même site, lampe proche de notre $\mathrm{n}^{\circ} 88$ : ibid., pl. XXXVII, $\mathrm{n}^{\circ} 1$.

146. SAlOMONSON J.W., Spätrömische rote Tonware, 1969, p. 97. Des échantillons de l'atelier d'Henchir es Srira déposés par Jean Deneauve au Centre Camille Jullian comprennent des exemplaires et un moule de lampe Atlante VIIIB.

147. Plus probablement byzantines : Nit:Rt: L., Cooking wares, 1986, p. 73 ; PavolINI C., Lucerne in Italia, 1998, p. 128-129 ; BarRal.D D. et al., Industrie céramique, 1998, p. 154.

148. Ibid., p. 154-156.

\section{Catalogue}

- 81 : lampe italique "Firmalampe "; timbre en relief [OC]TAVI ; pâte orange, granuleuse, petites incl. grises ; surface brun orangé. (Inv. 016.08)

- 82 : lampe africaine (?), type Deneauve VII (?) ; bandeau décoré d'une rangée d'oves en creux, disque orné d'une rosace (?) ; pâte beige, compacte et dure, nombreuses incl. rouges, grises et noires ; traces d'engobe brun. (Inv. 016.09) - 83 : lampe africaine type Deneauve X A ; bandeau décoré d'une rangée de feuilles d'acanthe, trou de remplissage et trou d'évent sur le disque décoré d'un personnage en pied vu de face, ensemble proche de Deneauve 1974 , p. 213 , $\mathrm{n}^{\circ} 1050$ et pl. XCV ; pâte beige orangé, compacte, nombreuses incl. blanches, grises et noires; engobe jaune orangé. (Inv. 038.01)

- 84 : lampe en sigillée africaine C (?), type Atlante I (?); bandeau décoré d'une mouluration à deux cannelures ; pâte beige orangé, homogène, sans incl. ; vernis orange, satiné, lisse. (Inv. 035.05)

- 85 : lampe en sigillée africaine C (?), type Atlante IV A (?) ; bandeau décoré d'une mouluration à trois cannelures ; pâte orange, homogène, compacte, sans incl. ; vernis orange, satiné, légèrement écaillé. (Inv. 029.04)

- 86 : lampe en sigillée africaine C (?), type Atlante IV B (?) ; bandeau décoré d'une moitié de palme, séparé du disque, lui-même bordé d'une palme, par une nervure en fort relief ; pâte brune, très compacte ; vernis orange sombre, satiné, très lisse ; ensemble de facture très soignée. (Inv. 042.05)

- 87 : lampe en sigillée africaine de type indéterminé ; bandeau décoré de globules, motif illisible car trop fragmentaire sur le disque ; pâte orange, granuleuse ; vernis orange, mat. (Inv. 004.05)

- 88: lampe en sigillée africaine de type Atlante VI (?); bandeau décoré de feuille(s) cordiforme(s) (?) en fort relief, motif illisible car trop fragmentaire sur le disque ; pâte orange, granuleuse ; vernis orange, écaillé. (Inv. 012.04)

- 89 : lampe en sigillée africaine de type Atlante VI (?) ; bandeau décoré d'une rouelle et d'une feuille (?), motif illisible car trop fragmentaire sur le disque ; pâte orange, granuleuse, petites incl. grises; vernis orange clair. (Inv. 042.04)

- 90 : lampe en sigillée africaine de type Atlante VI (?); bandeau décoré de feuilles ; pâte beige orangé, compacte ; engobe orange clair ; aspect très particulier, proche des productions de lampes tripolitaines. (Inv. 035.04) 


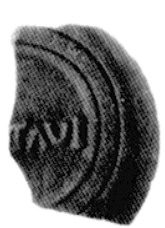

81

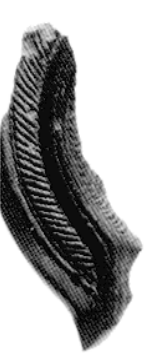

86

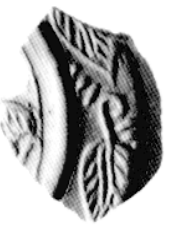

90

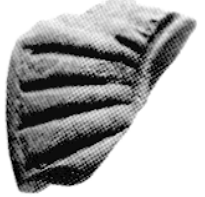

94

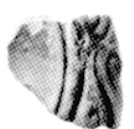

95

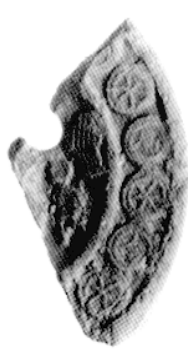

99

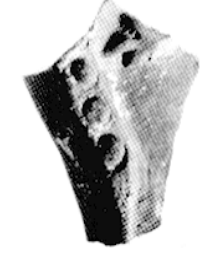

87

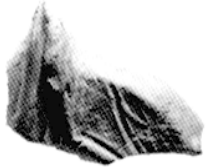

91

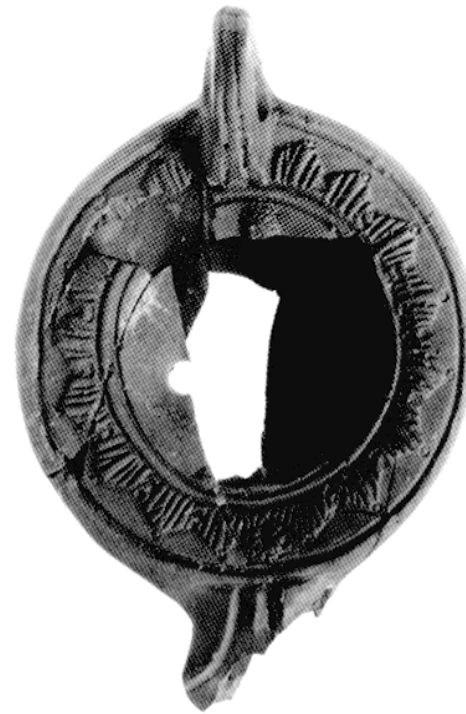

83
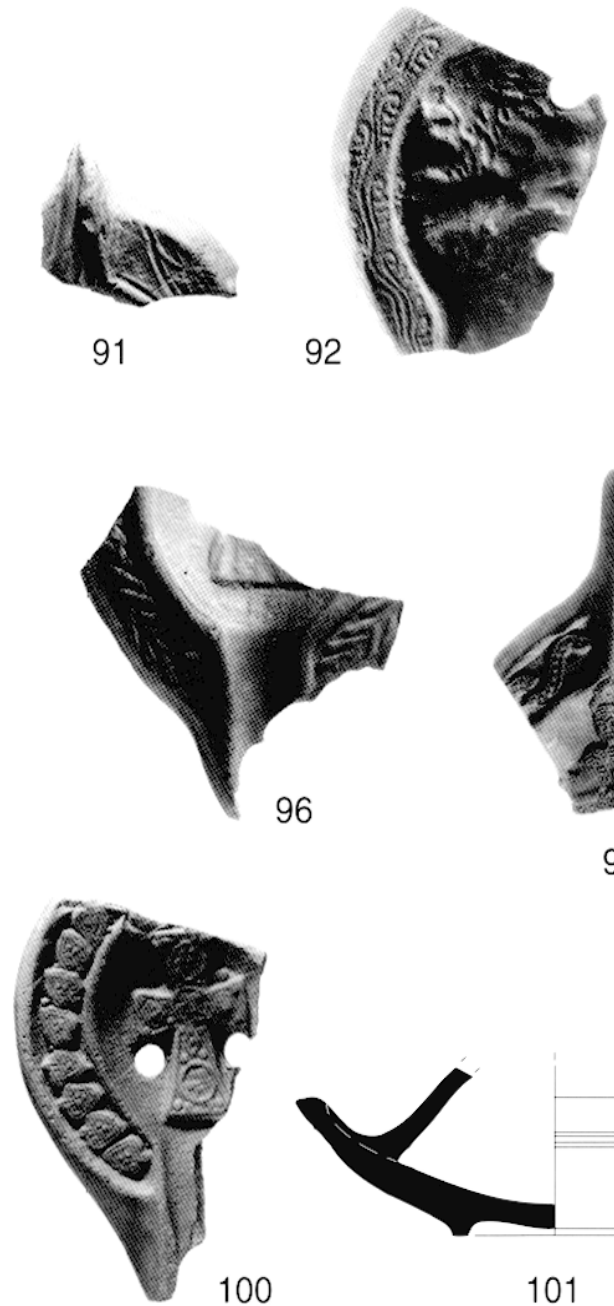

101

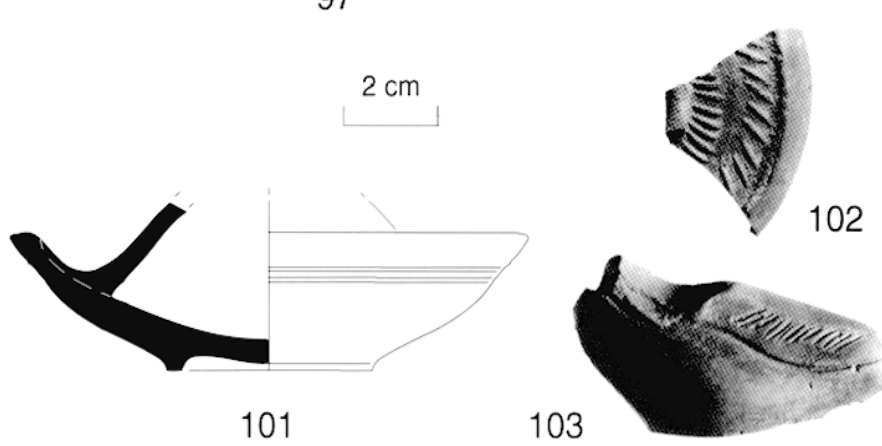

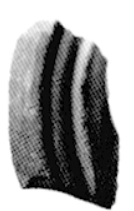

84

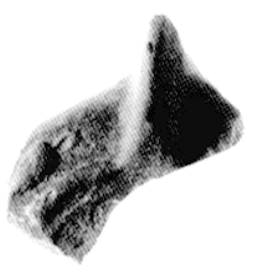

88
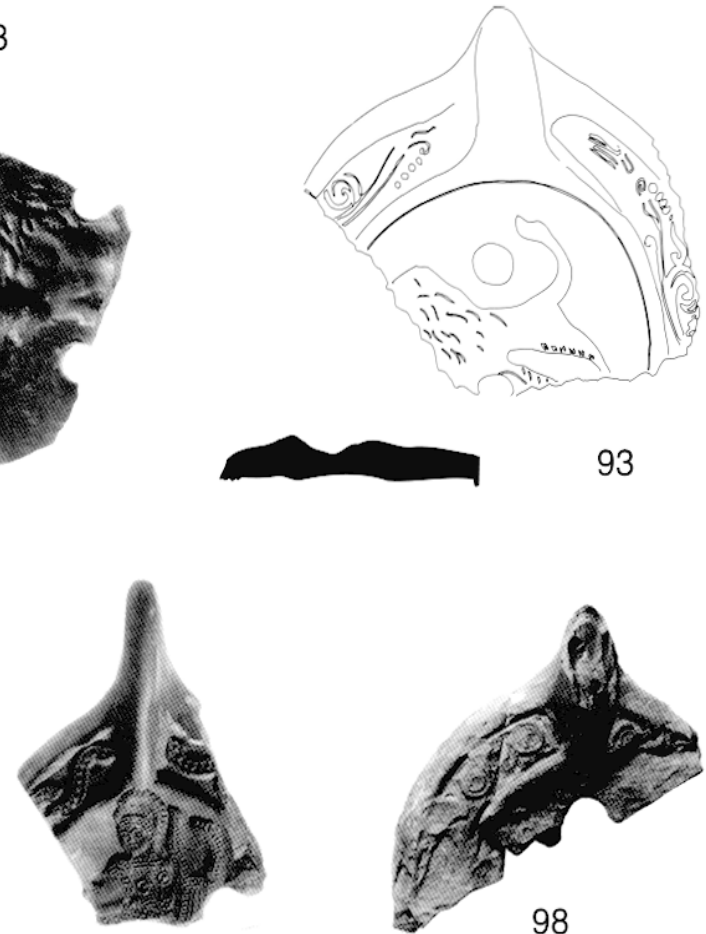

97

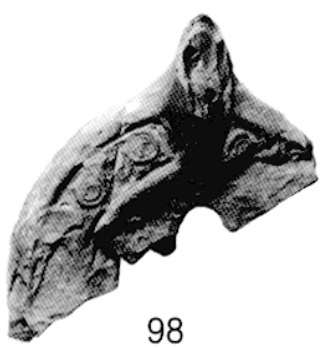

98

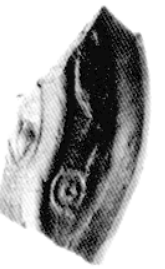

89

85

Fig. 9 : Lampes. (M. Bonifay ; photographies Chr. Durand) 
- 91 : lampe en sigillée africaine de type Atlante VI ; bandeau décoré d'un rinceau ; pâte brune, compacte ; vernis rouge orangé clair. (Inv. 005.04)

- 92 : lampe en sigillée africaine de type Atlante VI ; bandeau décoré d'un rinceau, lion bondissant à gauche sur le disque, trous de remplissage au-dessus et au-dessous de l'animal ; pâte orange, granuleuse ; vernis orange, mince. (Inv. 029.02)

-93 : lampe en sigillée africaine de type Atlante VI ; décor identique à la précédente ; pâte orange ; vernis orange, mince. (Inv. 001.03)

- 94 : lampe en sigillée africaine (?), "Melonenlampe "(?); pâte orange, granuleuse ; vernis orange, écaillé. (Inv. 016.10)

- 95: lampe en sigillée africaine, type Atlante VIII B ; bandeau décoré d'un rinceau ; pâte orange clair, vacuolaire, sans incl. ; vernis orange clair. (Inv. 032.05)

- 96 : lampe en sigillée africaine, type Atlante $\mathrm{V}$ dit " de Navigius "(?); bandeau décoré d'une palme ; pâte orange, granuleuse, sans incl.; vernis orange, mat, assez épais. (Inv. 042.03)

- 97 : lampe en sigillée africaine $\mathrm{C}$, type Atlante X (Hayes II A) ; bandeau décoré de motifs en S (Ennabli Q 1) formant une tresse, personnage casqué sur le disque, ensemble très proche d'Ennabli 1976, p. 66, n 157 et pl. VIII ; pâte orange, très fine ; vernis orange vif, satiné, très soigné. (Inv. 037.07)

- 98 : lampe en sigillée africaine C, type Atlante X (Hayes II A) ; bandeau décoré de cercles concentriques (Ennabli $\mathrm{E}$ 6 ?) ; pâte orange clair, fine ; vernis orange, écaillé. (Inv. 032.04)

- 99: lampe en sigillée africaine, type Atlante X (Hayes II B) ; bandeau décoré de disques Ennabli $\mathrm{F} 3$ alternant avec des disques Ennabli $\mathrm{F} 5$, sur le disque scène du sacrifice d'Abraham (?), décor identique à Ennabli 1976, p. 43, nos $14-15$ et pl. I ; pâte brun orangé, assez grossière ; engobe rouge rosé. (Inv. 070.05)

- 100 : lampe en sigillée africaine, type Atlante X (Hayes II B) ; bandeau décoré de fleurons cordiformes, sur le disque grande croix gemmée, décor proche d'Ennabli 1976, n 1072 et pl. LVII ; pâte rouge brique, dure, assez grossière; vernis rouge orangé, mat. (Inv. 078.05)

- 101 : lampe tournée en sigillée africaine, type Atlante XVI ; pâte orange clair, granuleuse ; vernis orange, mat. (Inv. 040.01)

- 102 : lampe "tripolitaine ", de type Atlante XIII ou XIV ; bandeau décoré d'une double rangée de traits parallèles en léger relief ; pâte orange à cœur gris, compacte et très dure ; engobe jaune orangé, satiné. (Inv. 004.04)

- 103 : figurine (?) en sigillée africaine C (?) : oiseau (?) ; pâte orange, fine, compacte, sans incl. ; vernis orange, satiné. (Inv. 037.09)

\section{La céramique culinaire (fig. 10)}

Les types classiques de la céramique culinaire africaine sont tous très bien représentés du sud au nord du littoral, que ce soient les formes de tradition punique ( $\mathbf{n}^{\text {os }}$ 104-105), ou bien les formes de l'époque romaine (nos 106-111, 205-210). Mais une catégorie particulière dont la distribution est concentrée sur le littoral de la Byzacène méridionale, essentiellement entre Sfax et Sousse ${ }^{149}$, a retenu notre attention. Il s'agit de grandes marmites à bord renflé à l'intérieur, évoquant la forme Hayes 23 (?) ; le vernis qui couvre l'intérieur du vase dessine de larges coulures à l'extérieur (nos 112-117; 258). Plusieurs arguments plaident en faveur d'une datation très tardive : deuxième moitié du $\mathrm{VI}^{\mathrm{e}}$ et $\mathrm{VII}^{\mathrm{e}} \mathrm{s} .{ }^{150}$.

\section{Catalogue}

- 104 : céramique culinaire africaine de tradition punique. forme Hayes 191. (Inv. 061.03)

- 105: céramique culinaire africaine de tradition punique, forme Hayes 198 (?). (Inv. 061.04)

- 106 : céramique culinaire africaine, forme Hayes 181 : pâte orange, grossière, vacuolaire, nombreuses incl. de quartz ; vernis orange à bandes lustrées (int.), partie supérieure ext. du vase noircie, bande grise au contact de l'empilement des vases lors de la cuisson. (Inv. 027.01)

- 107 : céramique culinaire africaine, forme Hayes 181 variante tardive (Ive $s . ?)$; vernis interne à bandes lustrées. (Inv. 055.06)

149. Sites $n^{0 s} 32,55,71,72,73,84,85,94$. John w. Hayes ma montré des fragments recueillis à Lamta-Leptiminus (mission tunisocanadienne dirigée par N. Ben Lazreg, L. Stirling et D. Stonc).

150. Forme présente dans le contexte homogène du site $n^{\circ} 85$ (voir infra) ; Rougga-Bararus : couches 10 et 13 (GIERY R.. Stratigraphie du forum, 1984, p. 96) ; Ksour Essaf, atelier damphore d'Henchir ech Chekaf : lors d'une visite du site en 1993. jai constaté la présence de cette forme en association avec les amphores tardives Keay LXI et LXII (cf. PEACoCK D.P.S., BEN LAZRF; . N. et BHJAOLI F., Roman Amphora Production, 1989), forme comparable (?) sur l'atelier de Rass Aïed avec les mêmes associations (Ihid.. fig. 9, $\mathrm{n}^{\circ} 7$ ) : Moknine, atelier d'amphores Keay LXI fouillé par .V. Ben Lazreg (inédit) : en association avec une forme Hayes 105. 

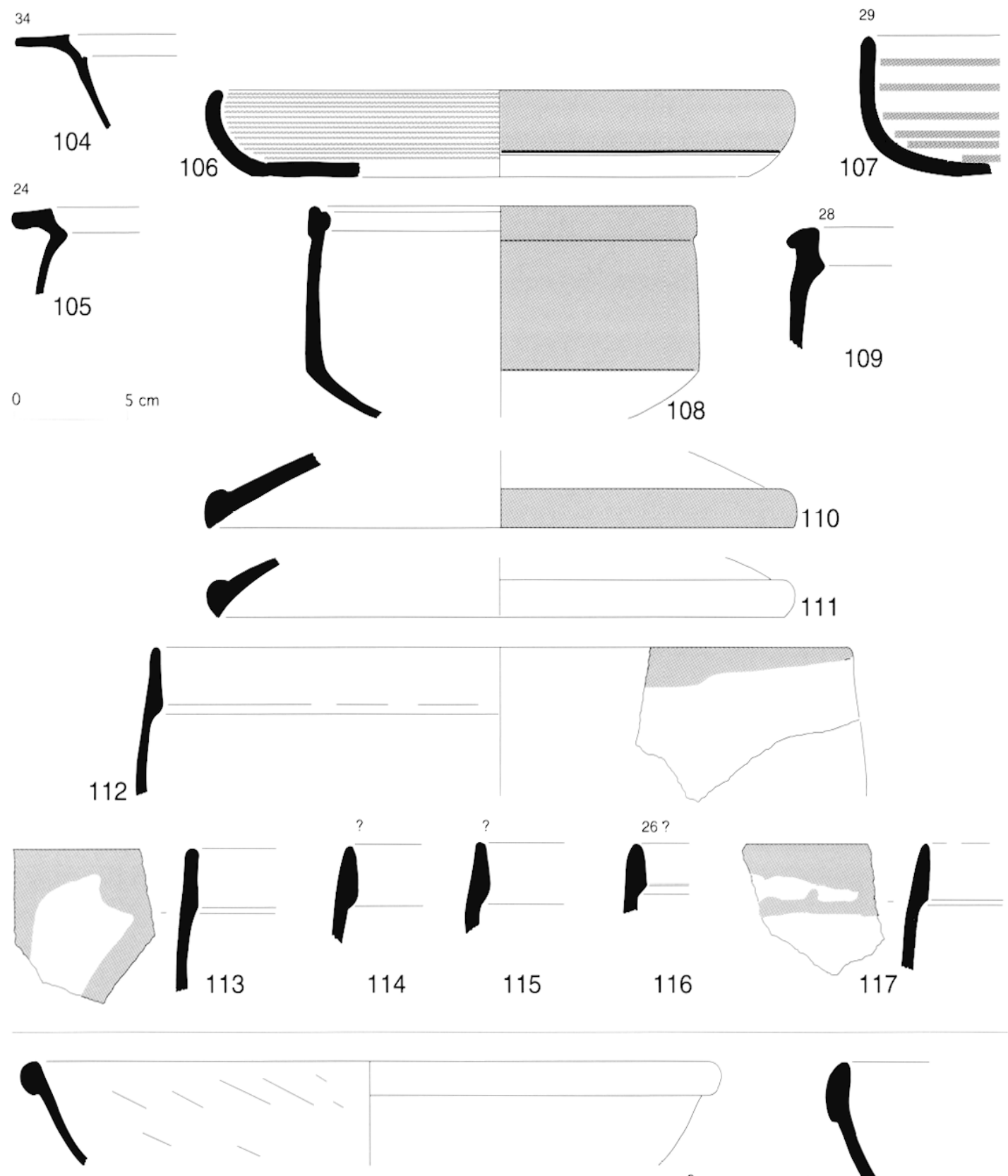

118
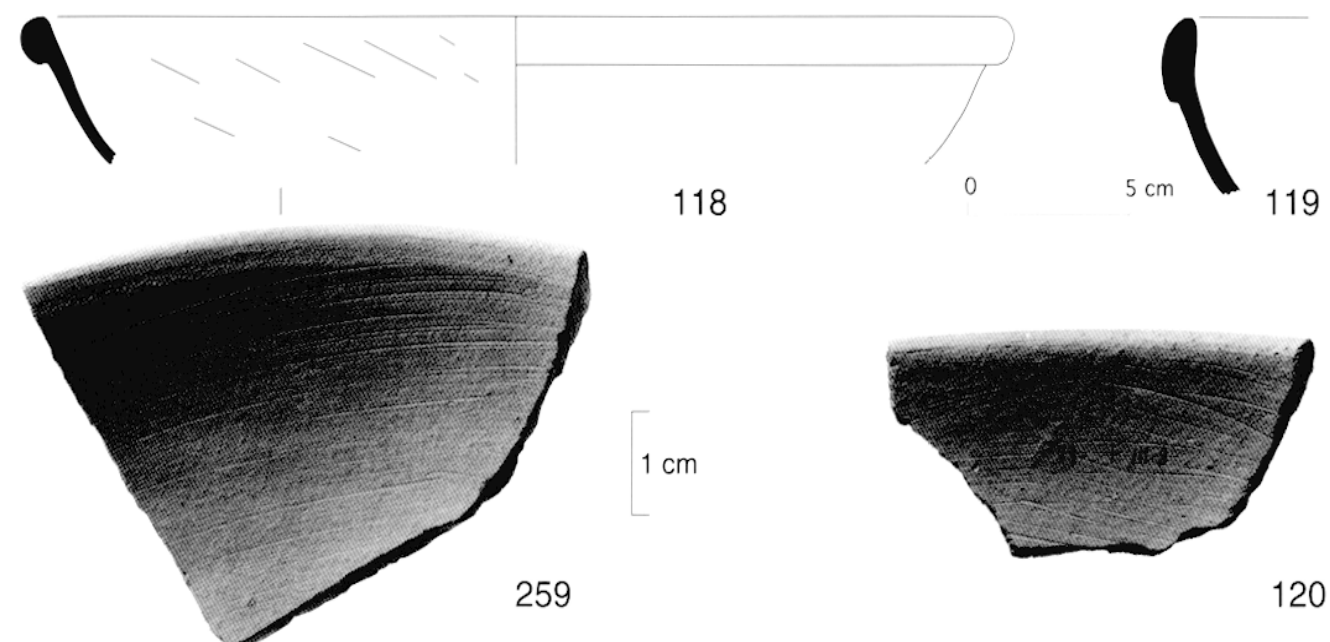

Fig. 10 : Céramiques culinaires africaines ( $\left.n^{\circ s} 104-117\right)$; céramiques communes africaines ( $\left.\mathrm{n}^{\circ \mathrm{s}} 118-119,259\right)$ ( $n^{\circ} 120$ : exemplaire de comparaison, Rougga-Bararus). (M. Bonifay) 
- 108 : céramique culinaire africaine, forme Sidi Jdidi $4{ }^{151}$, variante tardive (?) ; ext. noirci. (Inv. 145.02)

- 109 : céramique culinaire africaine, forme Hayes 183 variante. (Inv. 055.09)

- 110 : céramique culinaire africaine, forme Hayes 196 variante ; pâte rouge brique, compacte, nombreux microfossiles et incl. de quartz anguleux ; engobe rouge, mat, à l'ext. (Inv. 023.01)

- 111 : céramique culinaire africaine, forme Hayes 196 variante ; pâte comparable à celle du $n^{\circ} 110$; surface ext. grise. (Inv. 023.02)

- 112 : céramique culinaire africaine, forme dérivée de Hayes 23 (?) ; pâte orange, compacte, nombreuses petites incl. de quartz; engobe orange clair, mat à l'int., coulures à l'ext. (Inv. 078.08)

- 113 : céramique culinaire africaine, forme dérivée de Hayes 23 (?) ; pâte orange ; engobe brun à l'int., coulures sur la surface ext. jaune. (Inv. 084.02)

- 114 : céramique culinaire africaine, forme dérivée de Hayes 23 (?) ; pâte orange, granuleuse ; engobe orange vif à l'int., surface ext. marron-beige, rugueuse. (Inv. 094.06)

- 115 : céramique culinaire africaine, forme dérivée de Hayes 23 (?). (Inv. 055.10)

- 116 : céramique culinaire africaine, forme dérivée de Hayes 23 (?). (Inv. 094.07)

- 117 : céramique culinaire africaine, forme dérivée de Hayes 23 (?). (Inv. 084.01)

\section{La céramique commune (fig. 10 et 11)}

La céramique commune qui n'est pas, dans l'état actuel des recherches, un bon instrument de datation, n'a pas fait l'objet d'investigations très poussées sur le terrain. Là encore, l'accent a été mis sur les productions de la partie centro-méridionale du littoral où trois types de formes assez répétitives méritent qu'on s'y attarde un peu. La pâte habituelle de ces objets est orange sombre ou rouge brique, granuleuse, avec de nombreuses inclusions translucides (quartz) et de fréquents points de chaux. Un premier groupe est constitué par des plats profonds à lèvre en amande qui imitent ou tout au moins rappellent la forme de sigillée Hayes 103 (nos 118-120; 259). Ces vases portent parfois un engobe orange sombre très mat (comparable à celui des marmites $\mathbf{n}^{\mathbf{o s}} \mathbf{1 1 2 - 1 1 7}$ ) mais

151. BONIfaY M.. Observations préliminaires, 2004, p. 46-48 et fig. $19 . n^{\circ} 73$. en sont plus généralement dépourvus ; dans les deux cas l'intérieur est sommairement lissé avec un objet (un chiffon ?) qui a retenu certaines particules lourdes et provoqué de très caractéristiques rayures (photographies des $\mathbf{n}^{\text {os }} \mathbf{1 2 0}$ et 259). Cette forme est toujours rencontrée dans les contextes tardifs de Byzacène méridionale, dans la deuxième moitié du $\mathrm{VI}^{\mathrm{e}}$ et au $\mathrm{VII}^{\mathrm{e}} \mathrm{s} .{ }^{152}$. La deuxième forme, un bassin, est caractérisée par un bord renflé, grossièrement quadrangulaire, souligné intérieurement par un léger gradin (nos $121-123 ; \mathbf{2 6 0 - 2 6 1}$ ). Une restitution de cette forme a été proposée par Roger Guérył à partir du matériel de Rougga-Bararus (nos 124-125). Cette forme pourrait être contemporaine de la précédente ou légèrement plus tardive ${ }^{153}$. Enfin, il nous a semblé utile d'illustrer les formes centro-méridionales de bols à listel (nos 126-133) qui diffèrent quelque peu de celles reconnues à Carthage ${ }^{154}$. La céramique peinte est rare $\left(\mathbf{n}^{\circ} \mathbf{1 3 4}\right)$.

\section{Catalogue}

- 118 : céramique commune africaine, forme dérivée de Hayes 103 (?) ; pâte rouge brique, granuleuse ; surface grossièrement lissée (rayures obliques) à l'int. (Inv. 089.01) - 119 : céramique commune africaine, forme clérivée de Hayes 103 (?). (Inv. 055.08)

- 120 : céramique commune africaine, forme dérivée de Hayes 103 (?) ; pâte rouge brique, granuleuse ; surface grossièrement lissée (rayures obliques) à l'int., bord ext. noirci à la cuisson. (Bararus-Rougga, exemplaire de comparaison) - 121 : céramique commune africaine, bassin à bord renflé ; pâte orange ; surface orange à l'ext., bord jaune, intérieur brun. (Inv. 084.04)

- 122 : céramique commune africaine, bassin à bord renflé ; pâte orange, granuleuse, feuilletée ; surface orange, ext. du bord blanc crème. (Inv. 071.06)

- 123 : céramique commune africaine, bassin à bord renflé ; pâte identique à celle du nº 121 . (Inv. 084.03)

152. Elle est présente à Rougga-Bararus dans les couches 10,12 et 13 (cf. GIÉRY R., Stratigraphie du forum, 1984. p. 96) : cf. également le site homogène $n^{\circ} 85$.

153. Rougga-Bararus, couches 10 et 12 (Ihid., p. 96) ; ateliers d'Henchir ech Chekaf et Rass Aïed (P'EACOCK D.P.S., BFN LAZRF:; N. et Bi:jaOLI F., Roman Amphora Production. 1989, fig. 8. n 24 et fig. 9. $\left.\mathrm{n}^{\circ} 18\right)$ : atelier de Moknine. en association avec une sigillée africaine Hayes 105 et des amphores Keay LXI

154. Hayts J.W. Pottery report. 1978, fig. 20 : F(t.FORI) M. et PEAcock D.P.S.. Alenue Bourguiba. 1984, fig. 76 à 78. 

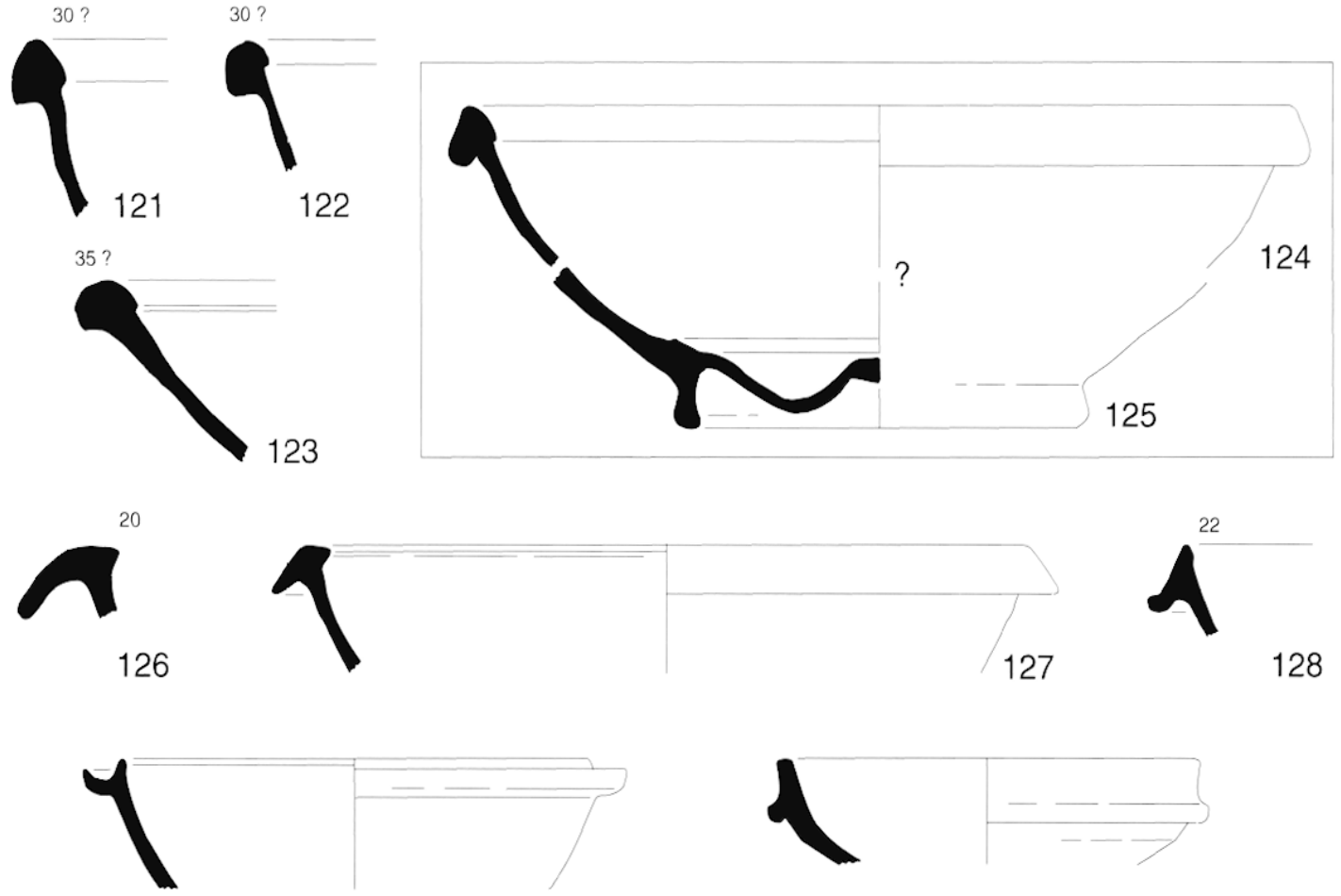

131

129
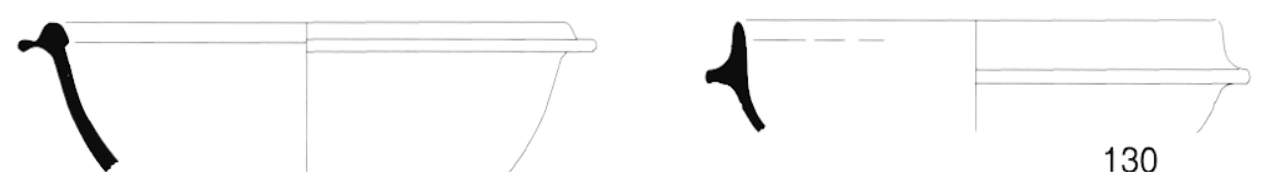

132
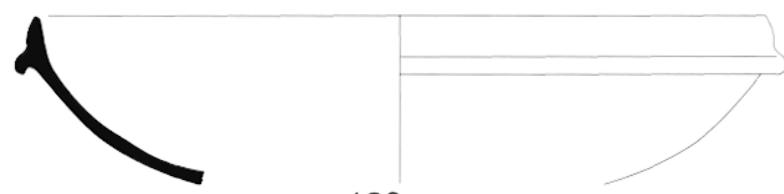

133

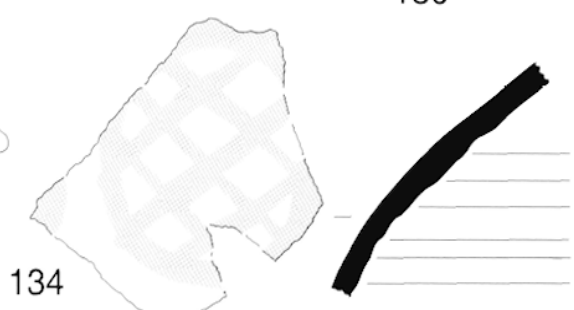

\section{0}
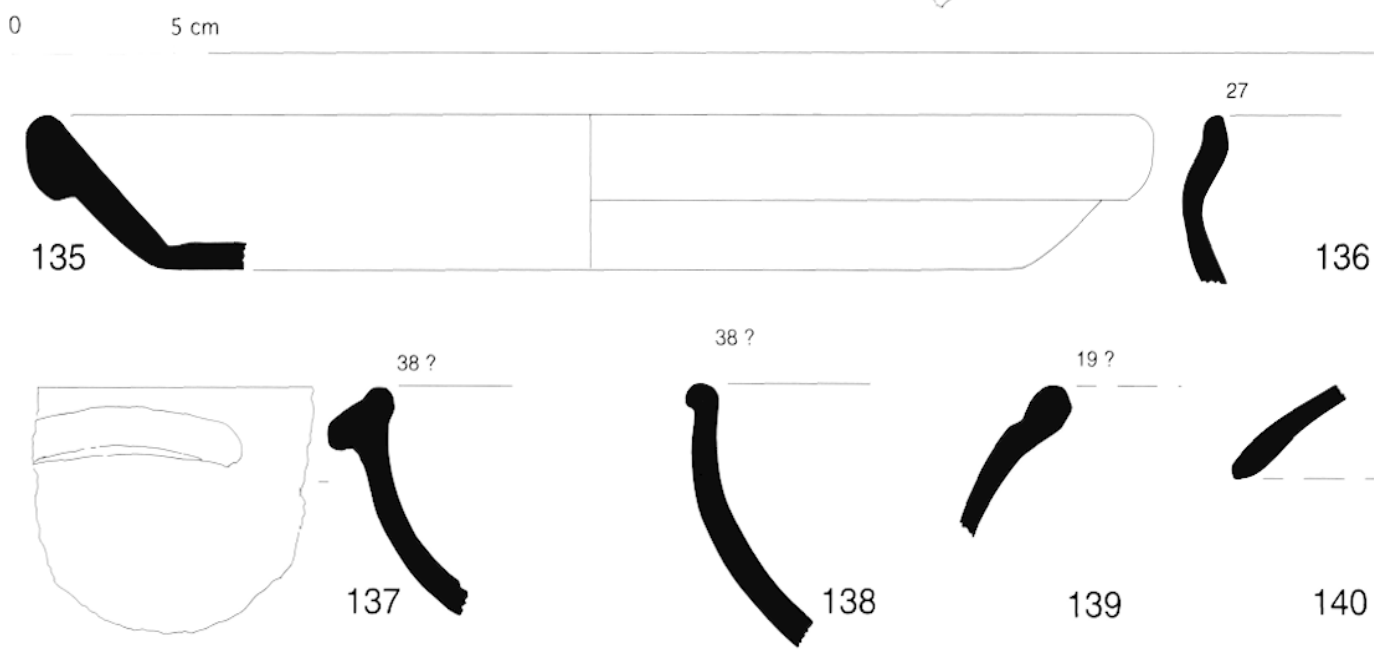

Fig. 11 : Céramiques communes africaines ( $\left.n^{\circ s} 121-123,126-133\right)$ ( $n^{0 s} 124-125$ : exemplaires de comparaison, Rougga-Bararus); céramique peinte ( $\left.\mathrm{n}^{\circ} 134\right)$; céramique modelée ( $\left.\mathrm{n}^{\cdots 1} 135-140\right)$. (M. Bonifay) 
- 124 : céramique commune africaine, bassin à bord renflé ; pâte rouge brique, granuleuse ; surface orange, ext. du bord jaune. (Bararus-Rougga, exemplaire de comparaison)

- 125 : céramique commune africaine, fond de bassin à bord renflé (?) ; pâte rouge brique, granuleuse. (BararusRougga, exemplaire de comparaison)

- 126 : céramique commune africaine, bol à marli courbe. (Inv. 037.15)

- 127 : céramique commune africaine, bassin ; pâte orange, granuleuse ; surface orange. (Inv. 078.03)

- 128 : céramique commune africaine, bol à listel. (Inv. $037.16)$

- 129 : céramique commune africaine, bol à listel ; pâte rouge brique, granuleuse, micro-fossiles, incl. de quartz ; surface orange. (Inv. 070.06)

- 130 : céramique commune africaine, bol à listel ; pâte orange, granuleuse ; surface orange. (Inv. 078.04)

- 131 : céramique commune africaine, bol à listel ; pâte rouge brique, compacte ; surface orange clair, engobe (?) mat à l'int. (Inv. 090.02)

- 132 : céramique commune africaine, bol à listel ; pâte rouge brique, feuilletée ; surface orange, engobe (?) mat à l'int. (Inv. 078.06)

- 133 : céramique commune africaine, bol à listel ; pâte rouge brique, compacte; surface orange. (Inv. 078.07)

- 134 : céramique commune africaine (?), vase fermé (?) ; pâte orange à cour beige, très compacte ; surface orange clair, décor peint en rouge. (Inv. 035.01)

\section{La céramique modelée (fig. 11)}

La diffusion de cette céramique ${ }^{155}$ sur le littoral concerne essentiellement les sites du Nord à occupation tardive. Certes les catégories de céramique modelée que l'on s'accorde à considérer comme importées de Pantelleria ${ }^{156}\left(\mathbf{n}^{\circ} 135\right)$ et d'Italie du Sud $\left(\mathbf{n}^{\circ} 136\right)$ sont bien présentes dans le Cap Bon et le golfe d'Hammamet ${ }^{157}$ durant toute l'époque romaine. De même on rencontre

155. Sites n“m 5,80,105, 143, 145, 146, 147, 162,164, 201, 203, 208 Les productions de Pantelleria sont attestées sur les sites soulignés.

156. Peacock D.P.S., Petrology and origins, 1984, p. 8-10.

157. À Nabeul : forme Fulford 1 (FutFoRi) M. et PEACOCK D.P.S., Avenue Bourguiba, 1984 , p. 157 et fig. 55) dans des contextes du III" au " s. ; à Hammamet-Pupput (BONIFAY M., Observations préliminaires. 2004. p. 54 et fig. 23) et à Hergla (documentation aimablement communiquée par Mme Ouertani) : marmite Sabratha 325 (DORF J., Coarse Pottery, 1989, fig. 66) dans des niveaux de la fin du II" s. et de la première moitié du $I I^{\circ} \mathrm{s}$. ; cette dernière forme est également attestéc à Lamta (DORE J.. First report. 1992, p. 142 et 144. ${ }^{\circ} .32$ ). en abondance dans le triangle Nabeul-Hammamet-Sidi Jdidi une catégorie de céramique modelée, sans doute de production locale, caractéristique des $\mathrm{ve}^{\mathrm{e}} \mathrm{vI}^{\mathrm{e}} \mathrm{s}$. (non illustrée $)^{158}$. Mais la proportion de céramique modelée d'origine indéterminée est, semble-t-il, plus importante sur les sites de la côte nord-ouest, dans des contextes des IVe-VII ${ }^{\mathrm{e}}$ s. (n ${ }^{\text {os }}$ 137-140).

\section{Catalogue}

- 135 : céramique modelée de Pantelleria (?), plat Fulford

1. (Inv. 145.03)

- 136 : céramique modelée d'Italie du Sud (?), bol Fulford

8. (Inv. 208.03)

- 137 : céramique modelée. (Inv. 201.02)

- 138 : céramique modelée. (Inv. 201.03)

- 139 : céramique modelée. (Inv. 208.01)

- 140 : céramique modelée. (Inv. 208.02)

\section{B - Les amphores}

Les principaux types d'amphores (fig. 12 à 15)

De manière tout à fait attendue, les prospections du littoral ont permis de rencontrer un nombre non négligeable de types d'amphores importées (fig. 12). Il s'agit tout d'abord d'amphores italiques d'époque républicaine, de type "gréco-italique " $\left(\mathbf{n}^{\circ} \mathbf{1 4 1}\right)$ ou Dressel 1, notées sur 7 sites échelonnés du sud au nord $^{159}$, ainsi que d'un bord de type Lamboglia $2^{160}$ avec un timbre $\operatorname{MEN}(\ldots)^{161}\left(\mathbf{n}^{\circ} \mathbf{1 4 2}\right)$. Les amphores orientales connaissent une très ample diffusion, surtout à époque tardive, avec les types Carthage LRA $1^{162}$ et LRA $2^{163}$ (nos 281 et 282 ) ; les types LRA 3 et 4

158. BEx ABEI) A., BoNifay M. et Fixot M., Note préliminaire, 1997, p. 18 et fig. 5 ; apparemment moins fréquente à Carthage, voir cependant HAYEs J.W., Pottery, 1976, p. 97 - LRCW 5 *; HAYES J.W., Pottery report, 1978, 1978, p. 78 et fig. 24 : - Grits Studdeds lids n; Fui.FORI M. et PEACOCK D.P.S., Avenue Bourguiba, 1984, p. 11, 161 et fig. 57 , $n^{\prime *} 13-17$ : * Hand-Made fabric 1.3 - : Nilru L., Cooking wares, 1986, p. 75 et fig. 22-23: LRCW 5 .

159. Sites nos 16, 21, 61, 79, 105, 181, 210.

160. Cipriano M. T. et Carre M.-B., Production et typologie, 1989.

161. On ne sait trop comment développer ce nom : MENOLA, mais le $\mathrm{N}$ est systématiquement rétrograde (BIANC-B!̣oN V. et al., RTAR II, 1998, n" 653-656), MENOP[...] (Ibid., n 657), MENA(NDE) : AMAR G. et Luol B., Estampilles, 1984, p. 158, n 60 (?).

162. Sites n"1 12bis, 32, 37, 42, 43, 44, 48, 54, 55, 68, 70, 73, 76, 78, $80,94,110,118,121,122,128,129.133,137,147,159,162,165,175$. 187, 190, 198, 200, 201, 203.

163. Sites $n^{\circ 1} 16,45,49,50,54,55,68,70,73,75,76,78,80,110$, 128, 133. 137, 139. 165, 198, 200, 207. 

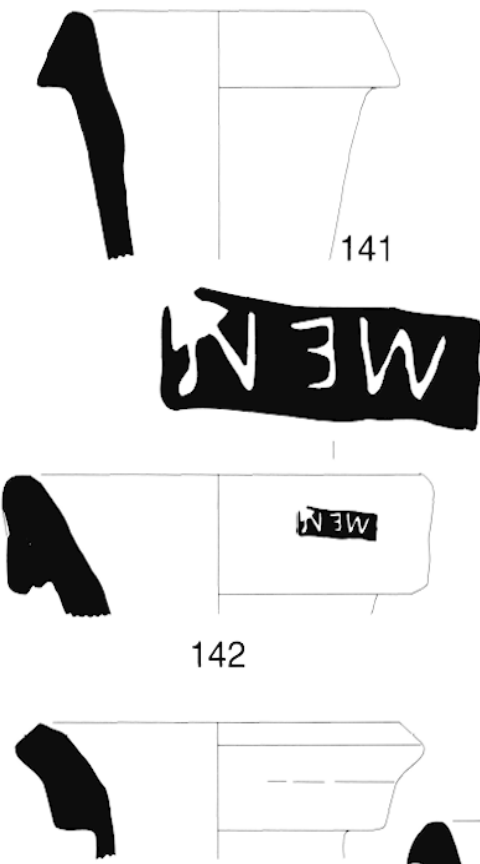

46
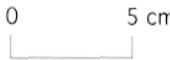

$+$
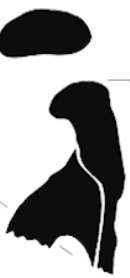

148

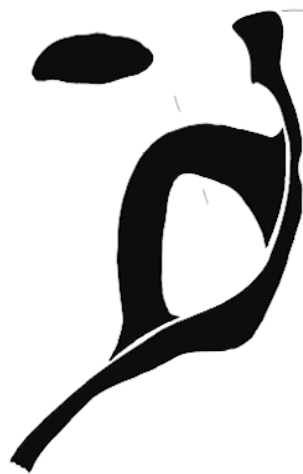

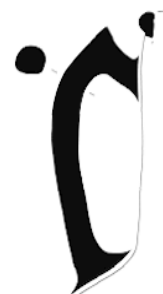

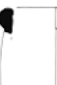
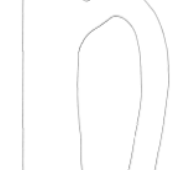

144

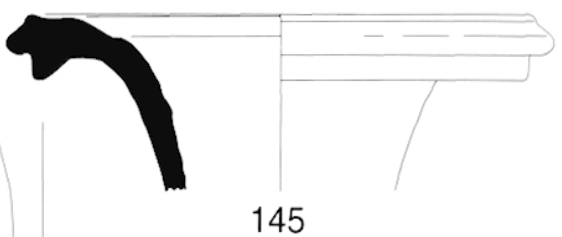

143

147

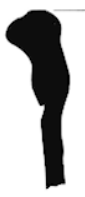

149

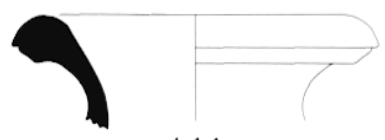

145

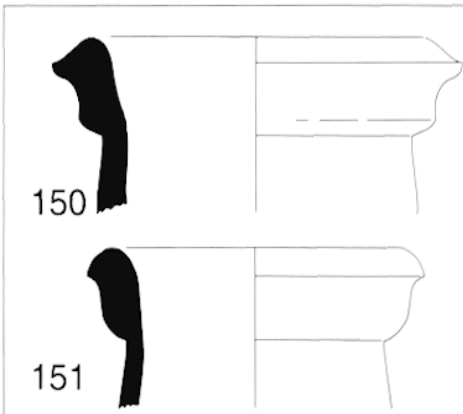

153

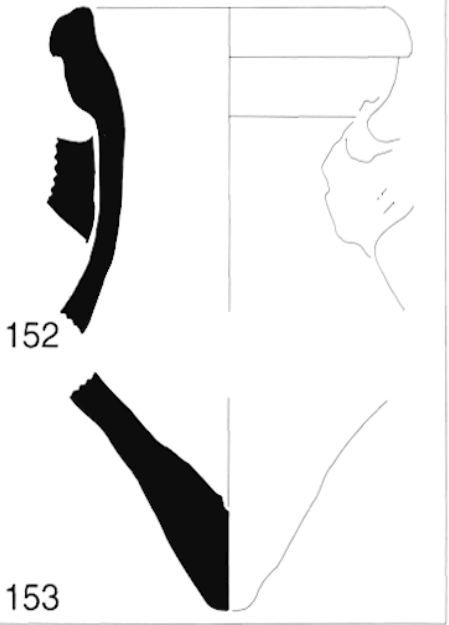

1

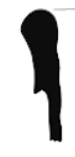

155

157

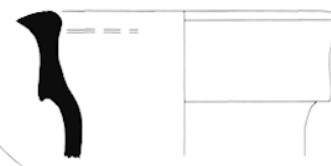

156

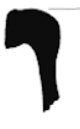

158

154

Fig. 12 : Amphores d'importation ( $\left.\mathrm{n}^{\prime \prime} 141-143\right)$; amphores puniques ( $\left.\mathrm{n}^{\prime *} 144-145\right)$; amphores tripolitaines ( $\left.\mathrm{n}^{\prime \prime} 146-149\right)$ ( $\mathrm{n}^{\mathrm{os}}$ 150-153 : atelier de Zian-Zitha) ; amphores de Byzacène méridionale ( $\mathrm{n}^{\mathrm{os}}$ 154-158). (M. Bonifay) 
sont également attestés mais nous avons choisi d'illustrer ici une amphore d'époque impériale de type Rhodienne 8 ( $\left.\mathbf{n}^{\circ} \mathbf{1 4 3}\right)$. Les amphores hispaniques sont peu nombreuses (un fragment de type Almagro 51A-B sur le site $\left.n^{\circ} 180\right)^{164}$.

Mais la grande majorité des amphores est d'origine africaine. Nous avons recueilli des fragments d'amphores de type punique (fig. 12) sur 14 sites $^{165}$. On observe une concentration du type Maña $D$ dans le Cap Bon ${ }^{166}$ mais les types Maña C1/2 $\left(\mathbf{n}^{\circ} \mathbf{1 4 4}\right)$ ou $\mathrm{C} 2\left(\mathbf{n}^{\circ} \mathbf{1 4 5}\right)$ sont les plus fréquents ${ }^{167}$. On doit cependant garder à l'esprit que beaucoup de ces amphores sont en fait postérieures à la chute de Carthage ${ }^{168}$. Les amphores "tripolitaines "(fig. 12) sont localisées dans la partie sud du littoral169. Nous avons attribué la plus grande partie des bords au type Tripolitaine III (nos 147-149) dont nous connaissons un atelier peu éloigné des côtes, à la périphérie de la ville antique de Zian-Zitha ${ }^{170}$. En dehors de quelques fragments d'anses bifides identifiées comme des imitations de Dressel 2/4 (type Schöne-Mau XXXV ?) ${ }^{171}$, on trouve sur cet atelier de nombreuses variantes d'amphores à bord à double gradin ( $\mathbf{n}^{\text {os }}$ 150-153) ainsi que des brûle-parfums et des mortiers à marli courbe et fond garni de grains de basalte. Le type Keay VIII B (nos 154-156), caractéristique de la seconde moitié du ve $\mathrm{s} .{ }^{172}$, possède de fortes analogies typologiques avec les amphores tripolitaines (fig. 12). On le rencontre en forte proportion sur le site $n^{\circ} 54$ (Onga) où l'on soup-

164. Une amphore Almagro 50 est exposée au Musée de Sfax. Dans l'usine de salaison de poissons de Nabeul, un certain nombre de cuvettes de nettoyage sont constituées d'amphores Dressel 20 dont on a oté le col (cf. Sim L., Bonifay M. et Trolisset P., Lusine de salaison, 1999).

165. Sites $n^{\circ \times 21,} 11,57,58,105,155,161,164,165,181,186,188$, $193,210$.

166. Sites $n^{\circ} 155,161,164,165,188$. À Nabeul, cette amphore est fréquente dans une couche d'incendie du $\operatorname{lif}^{\mathrm{C}} \mathrm{s}$. av. J.-C. (cf. SIIM L., Bonilay M. et Trotsst: P., Lusine de salaison, 1999).

167. Sites n's 41, 57, 58, 105, 164, 181, 186, 188, 210.

168. Cf. RAMON TORRis J., Anforas fenicio-punicas, 1995, p. 293-296 Isur les amphores de tradition punique, voir désormais : MARTINKItcul:R S., Die Füllung eines frühkaiserzeitlichen Pozzo, 1999].

169. Sites $n^{\circ \cdots 1} 1,5,16,22,32,61$.

170. Visites du site en 1990 et 1993 en compagnie du responsable du site. M. Ali Drine.

171. BEx LAZREc; N. et al.. Salsamenta, 1995, p. 131. Voir infra, atelier de Guellala.

172. BONIFAY M. et PIERI D.. Amphores. 1995, p. 100. çonne qu'il a pu être fabriqué173 ; dans la même pâte, on trouve également sur ce site des fragments du type Keay LIX ( $\left.\mathbf{n}^{\circ} \mathbf{1 5 8}\right)$, datable de la fin du Ive $s$. ou du début du ve $\mathrm{s}$. Le type Keay VIII B est probablement une évolution du type Keay LIX.

Les amphores africaines "classiques " sont omniprésentes sur le littoral (fig. 13) : type Africaine I ( $\mathbf{n}^{\circ} 159$ ) et II ( $\mathbf{n}^{\text {os }} 160$ et 163 ), de même que les amphores cylindriques de moyennes dimensions : types Keay XXV.1 ( $\left.\mathbf{n}^{\circ} \mathbf{1 6 4}\right), \mathrm{XXV} .2$ (non illustré) et XXV.3 ( $\left.{ }^{\circ} \mathbf{1 6 5}\right)$. Une variante particulière d'Africaine II ( $\mathbf{n}^{\circ} \mathbf{1 6 1}$ ), à bord en "S ", est assez fréquente (Peacock et al. 1990, p. 82 : "pseudo Tripolitanian types". Voir également Keay 1984, type Vbis); elle trouve comparaison à $\mathrm{El} \mathrm{Jem}\left(\mathbf{n}^{\circ} \mathbf{1 6 2}\right)^{174}$. Une amphore mérite qu'on s'y attarde un peu plus : le type Keay XXXV B (nos 166-169: 170 ?), bien connu sur le littoral septentrional de la Méditerranée, de la Catalogne à la Ligurie, au milieu du ve s. ${ }^{175}$, est distribué sur toute la côte est, avec une densité particulière dans le Cap Bon ${ }^{176}$. Or, on connaît maintenant un atelier, situé au lieu-dit Sidi Zahruni à Beni Khiar ${ }^{177}$, à $6 \mathrm{~km}$ au nord de Nabeul, qui a produit entre autres $\left(\mathbf{n}^{\circ} \mathbf{1 7 2}\right.$ : Keay LV A) ce type d'amphore ( $\left.\mathbf{n}^{\circ} \mathbf{1 7 1}\right)$. L'homogénéité de la pâte des exemplaires tunisiens ${ }^{178}$ et de ceux du Midi de la France ${ }^{179}$ nous laisse envisager qu'il s'agit là d'un des principaux ateliers de cette amphore (voir infra, la contribution de $\mathrm{Cl}$. Capelli). Parmi les types de la deuxième moitié du ve $s$., on mentionnera le type Keay LVII ( $\left.\mathbf{n}^{\circ} \mathbf{1 7 3}\right)$ présent sur un site de la côte nord et le type Albenga 11-12180 (nos 175-176) sur des sites de Byzacène méridionale.

173. Nejib Ben Lazreg est à l'origine de cette hypothèse. Lors d'une visite sur le site en 1993, nous avons observé des zones cendreuses, des tessons surcuits mais malheureusement aucun véritable déchet de cuisson.

174. Dépôt de fouille. Je remercie M. Hédi Slim et Mme Latifa Slim de m'avoir autorisé à examiner et dessiner ces objets en 1993.

175. Rfmola J. A. et Ablillo A., Les àmfores, 1989 ; BONifay M., Observations, 1986 ; SANTAMaria Cl., Dramont E, 1995.

176. Sites $\mathrm{n}^{\mathrm{\prime}} 1,16,32,37,53,55,70,122, \underline{133}, \underline{138}, \underline{139}, \underline{140}, \underline{142}$. $\underline{147}, \underline{148}, \underline{151}, 165,175$.

177. Je remercie M. Azaïez Antitf, Conservateur du Musée de Sousse et originaire de Beni Khiar, de mavoir signalé cet atelier que jai visité en 1996, avec l'autorisation de M. Hédi Slim Ivoir désormais : GHALIA T., BONIFAY M., CAPFLLl Cl., Sidi Zahruni, à paraitre].

178. Sauf $n^{\circ} 170$ : type douteux.

179. Exemplaires de Port-Vendres, Marseille et Dramont E.

180. BONIFAY M. et PIERI D.. Amphores. 1995. p. 102. 
Les amphores africaines de grandes dimensions des $\mathrm{VI}^{\mathrm{e}}$-VII ${ }^{\mathrm{e}} \mathrm{s}$. (fig. 14) sont très bien diffusées ${ }^{181}$ avec deux types principaux : le type Keay LXII dans sa variante A (nos 177-179), caractéristique du $\mathrm{VI}^{\mathrm{e}} \mathrm{s}$., à laquelle succède une variante (nos 180-181) de la fin du $\mathrm{VI}^{\mathrm{e}} \mathrm{s}$. et de la première moitié du VII $\mathrm{e}$ s., et le type Keay LXI dans sa variante C (n ${ }^{\text {os }} \mathbf{1 8 2 - 1 8 3}$ ) contemporaine de la précédente, puis A (nos 184-185) au vile $\mathbf{s}$. Il semble que ces amphores ainsi que les nombreux exemplaires retrouvés à Marseille ${ }^{182}$ proviennent en grande partie ${ }^{183}$ d'un atelier majeur situé dans l'arrière-pays de Salakta : Henchir ech Chekaf ${ }^{184}$; d'autres (probablement le $\mathbf{n}^{\circ}$ 177) sont produits sur les ateliers de la région de Nabeul. Moins connu, on remarque dans la région de Rass Botria (Acbolla) un type d'amphore ${ }^{185}$, probablement assimilable au type Keay XXXIV, caractérisé par une lèvre triangulaire pendante (nos 186-188; 191-194 ?) et un fond à renflement conique $\left(\mathbf{n}^{\circ} 189\right)$ et qui trouve comparaison à Rougga-Bararus ( $\mathbf{n}^{\circ} \mathbf{1 9 0}$ ) dans un contexte de l'Antiquité tardive ${ }^{186}$. De fait cette amphore est généralement associée, sur le littoral, à du matériel des $\mathrm{VI}^{\mathrm{e}}$-VII $\mathrm{e}$. (voir infra, $\mathbf{n}^{\circ} 269$ ).

Enfin, certains sites du golfe d'Hammamet ${ }^{187}$ livrent un type d'amphore très particulier, à anses sur l'épaulement, dans la tradition des amphores puniques (fig. 15). Les fouilles de Sidi Jdidi-Aradi ${ }^{188}$ et plus récemment celles d'Hammamet-Pupput ${ }^{189}$ ont permis de tracer un cadre typologique provisoire en trois types. Le type 1 (nos 195 et 200) est caractérisé par un

181. Sites $\mathrm{n}^{\circ} 21,49,50,51,54,65,70,71,72,73,75,76,78,80,85$, $86,90,94,95,99,101,105,108,109,110,111,121,129,131,134,137$, 139, 142, 144, 175, 203 bis.

182. BONIFAY M. et PIIRI D., Amphores, 1995.

183. Pâte compacte avec peu d'incl. (translucides et brunes) mais de gros grains de chaux très clairsemés qui font parfois éclater la surface.

184. Pyacock D.P.S., Ben Lazreg N. et BejaOUi F., Roman Amphora Production, 1989, p. 184-186 et fig. 7-8. Nejib Ben Lazreg a fouillé en 1993 un atelier à Moknine qui a produit des amphores du type Keay LXI.

185. Pâte compacte sans grosses inclusions.

186. GUÉRY R., Stratigraphie du forum, 1984, p. 96 : couche 13.

187. Notamment le site $n^{\circ} 127$ bis.

188. BiN ABED A., Bonifay M. et Fixot M., Note préliminaire, 1997, p. $19-21$.

189. Fouilles d'urgence de la nécropole de Pupput, dirigées par Mme A. Ben Abed et M. M. Griesheimer. Étude de la céramique par M. Bonifay, T. Mukai, D. Pieri et J.-Chr. Treglia. [Voir désormais : BONIFAY M., Amphores de tradition punique, 2004]. bord déversé vers l'extérieur (à collerette) et mouluré (comme les amphores puniques Maña $\mathrm{C} 2 \mathrm{~b}$ ), un long col, une panse cylindrique portant deux petites anses nervurées, et un fond tronconique creux et court. Le type $2^{190}$ garde en commun avec le précédent un fond creux mais plus conique et des anses marquées longitudinalement par une profonde rainure. Le bord, en revanche, est très différent : la lèvre, plus courte et de section massive, est repliée horizontalement vers l'extérieur ( ${ }^{\circ} 196$ : variante tardive). Le type $3^{191}$ est caractérisé par un bord en bandeau, le plus souvent mouluré (nos 197-198). L'allure générale de ce conteneur reste assez proche de celle des types 1 et 2 , malgré la disparition des nervures longitudinales sur les anses et le remplacement du fond conique creux par des fonds coniques ou tronconiques pleins. Ces amphores " du golfe d'Hammamet "s'échelonnent du $\mathrm{II}^{\mathrm{e}}$ au $\mathrm{VII}^{\mathrm{e}}$ s. ${ }^{192}$.

\section{Catalogue}

- 141 : amphore gréco-italique. (Inv. 061.06)

- 142 : amphore italique, type Lamboglia 2 ; pâte saumon, vacuolaire, grains rouges (chamotte) et noirs ; surface jaune, timbre sur le bord : MEN dans cartouche rectangulaire. (Inv. 105.01)

- 143 : amphore rhodienne, type Empereur 8 ; pâte beige légèrement rosé, très fine, sableuse ; surface beige-blanc. (Inv. 118.01)

- 144 : amphore punique, type Maña C1/2 (type Ramon 7.3.1.1). (Inv. 021.08)

- 145 : amphore punique, type Maña C2B (type Ramon 7.4.3.3). (Inv. 021.05)

-146 : amphore africaine de type Tripolitaine I (?). (Inv. 011.07)

- 147 : amphore africaine de type Tripolitaine III. (Inv. 001.06)

- 148 : amphore africaine de type Tripolitaine III. (Inv. 001.08)

- 149 : amphore africaine de type Tripolitaine III. (Inv. 001.10)

190. BFא ABl:I) A., BONIFAY M. et FIXOT M., Note préliminaire, 1997, fig. $7, \mathrm{n}^{\circ} 41$.

191. Ihid., fig. 6. $n^{\circ} 31$

192. Type $1: \mathrm{II}^{\mathrm{c}} \mathrm{s}$. - premier quart III" s. ; type $2: \mathrm{III}-$ milieu ve s. ;

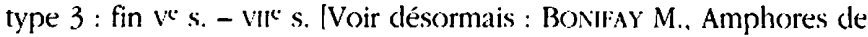
tradition punique, 2004]. 


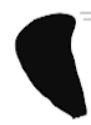

159

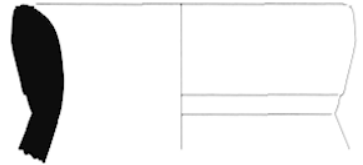

160
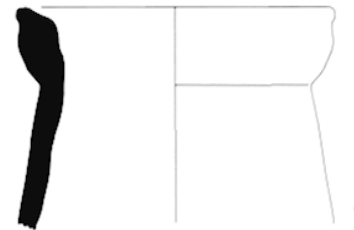

161

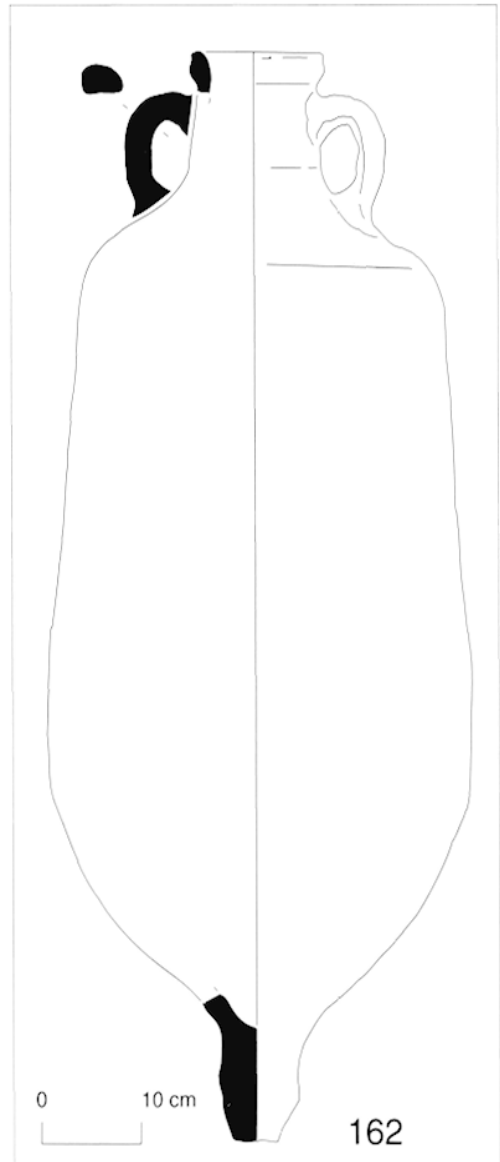

0

174

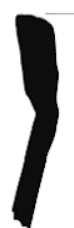

163

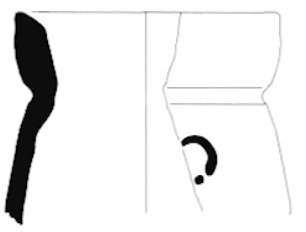

164
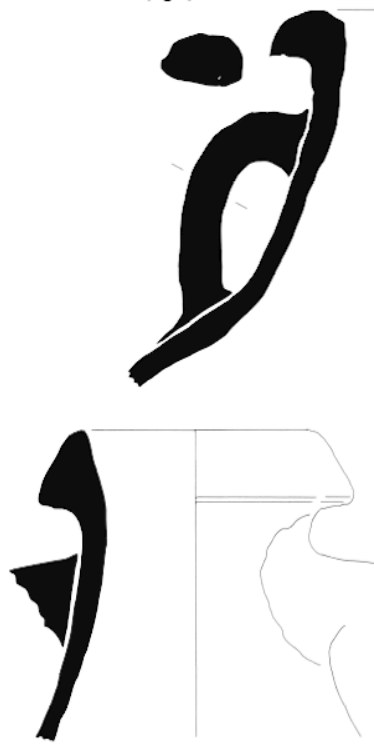

167

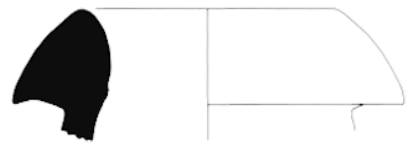

170

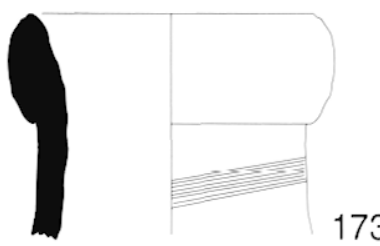

173

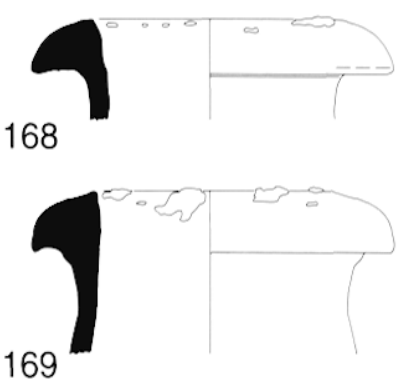

169

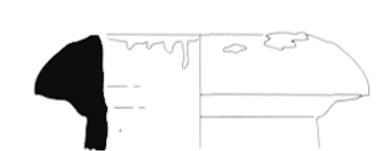

171
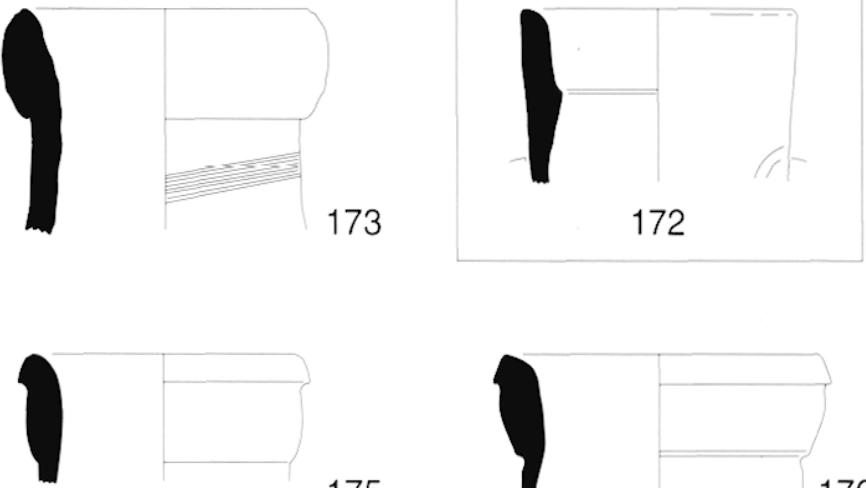

175
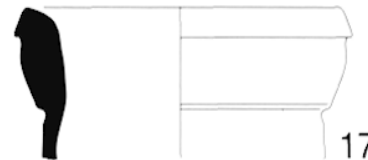

Fig. 13 : Amphores africaines classiques ( $\left.\mathrm{n}^{\circ \mathrm{s}} 159-161,163\right)\left(\mathrm{n}^{\circ} 162\right.$ : exemplaire de comparaison, El Jem) ; amphores africaines cylindriques de moyennes dimensions ( $\left.\mathrm{n}^{\text {os }} 164-165\right)$; amphores africaines cylindriques de grandes dimensions type Keay XXXV B ( $n^{\circ \infty} 166-169,170$ ?) ( $n^{\circ} 171$ : atelier de Sidi Zahruni) ; autres amphores cylindriques de grandes dimensions ( $\left.\mathrm{n}^{\circ \mathrm{s}} 173-176\right)\left(\mathrm{n}^{\circ} 172\right.$ : atelier de Sidi Zahruni) ; "spatheion" (?) ( $\left.\mathrm{n}^{\circ} 174\right)$. (M. Bonifay) 
- 150 : amphore africaine de type Tripolitaine I ou III ; pâte beige-jaune; surface beige gris. (Zitha-Zian, ateliers (?), exemplaire de comparaison)

- 151 : amphore africaine de type Tripolitaine I ou III ; pâte orange, fine, très petiles incl. blanches, surface beige-gris. (Zitha-Zian, ateliers, exemplaire de comparaison)

- 152 : amphore africaine de type Tripolitaine III ; pâte orange rosé, fine ; surface jaune pâle. (Zitha-Zian, ateliers, exemplaire de comparaison)

- 153 : amphore africaine de type Tripolitaine I ou III ; pâte orangé-brun, fine ; surface jaune. (Zitba-Zian, ateliers, exemplaire de comparaison)

- 154 : amphore africaine, type Keay VIII B ; pâte orange, très compacte; surface beige à l'ext. orange à l'int. (Inv. 054.11)

- 155: amphore africaine, type Keay VIII B ; pâte orange clair, compacte ; surface grise à l'ext., orange à l'int. (Inv. 054.03)

- 156 : amphore africaine, type Keay VIII B ; pâte orange clair, compacte ; surface beige à l'ext., orange à l'int. (Inv. 054.01)

- 157 : amphore africaine, type indéterminé ; pâte orange, compacte, granuleuse, nombreux grains blancs et translucides ; surface beige à l'ext., orange à l'int. (Inv. 073.04)

- 158 : amphore africaine, type Keay LIX ; pâte orange clair, granuleuse ; surface beige à l'ext., orange clair à l'int. (Inv. 054.05)

- 159 : amphore Africaine I B, surcuite ; pâte jaune verdâtre, vacuolaire, grosses incl. blanches et translucides ; surface gris verdâtre, poreuse. (Inv. 042.06)

- 160 : amphore Africaine II B " con gradino "; pâte rouge brique ; surface marron-gris. (Inv. 001.05)

- 161 : amphore Africaine II B, variante Keay V Bis ; pâte rouge brique, granuleuse, dure; surface blanche, usée. (Inv. 001.04)

- 162 : amphore Africaine II B, variante Keay V Bis ; pâte orange ; surface blanchâtre. (Dépôt de fouille d'El Jem, exemplaire de comparaison)

- 163 : amphore Africaine II D ou cylindrique de moyennes dimensions, type Keay XXV.1. (Inv. 055.11)

- 164 : amphore africaine cylindrique de moyennes dimensions, type Keay XXV.1. (Inv. 204.02)

- 165 : amphore africaine cylindrique de moyennes dimensions, type Keay XXV.3. (Inv. 001.09)

- 166 : amphore africaine cylindrique de grandes dimensions, type Keay XXXV B ; pâte orange ; surface gris foncé avec traces verdâtres. (Inv. 151.01)
- 167 : amphore africaine cylindrique de grandes dimensions, type Keay XXXV B ; pâte orange, compacte, grosses incl. de quartz ; surface ext. jaune usée, int. orange. (Inv. 145.01)

- 168 : amphore africaine cylindrique de grandes dimensions, type Keay XXXV B ; pâte orange, compacte, petites incl. de quartz ; surface jaune violacée usée à l'ext., orange à l'int., bavures d'argile au sommet du bord. (Inv. 140.02) - 169 : amphore africaine cylindrique de grandes dimensions, type Keay XXXV B ; pâte orange, compacte, minuscules incl. de quartz; surface jaune usée, bavures d'argile au sommet du bord. (Inv. 138.01)

- 170 : amphore africaine cylindrique de grandes dimensions (?), type Keay XXXV B variante (?) ; pâte rouge brique, sableuse, gros points de chaux, incl. de quartz et grises; surface orange. (Inv. 084.05)

- 171 : amphore africaine cylindrique de grandes dimensions, type Keay XXXV B ; pâte orange, compacte ; surface blanc crème, bavures d'argile au sommet du bord. (Beni Khiar, atelier de Sidi Zahruni, exemplaire de comparaison) - 172 : amphore africaine cylindrique de grandes dimensions, type Keay LV ; pâte rouge brique, compacte ; surface jaune violacée. (Beni Khiar, atelier de Sidi Zahruni, exemplaire de comparaison)

- 173 : amphore africaine cylindrique de grandes dimensions, type Keay LVII ; décor peigné. (Inv. 165.02)

- 174: amphore africaine de type spatheion (?). (Inv. 078.15)

- 175 : amphore africaine cylindrique de grandes dimensions, type Albenga 11-12 (?). (Inv. 070.08)

- 176 : amphore africaine cylindrique de grandes dimensions, type Albenga 11-12 (?). (Inv. 078.11)

- 177 : amphore africaine cylindrique de grandes dimensions, type Keay LXII A ; pâte beige orangé, très granuleuse ; surface beige usée. (Inv. 108.01)

- 178 : amphore africaine cylindrique de grandes dimensions, type Keay LXII A. (Inv. 078.10)

- 179 : amphore africaine cylindrique de grandes dimensions, type Keay LXII ; pâte orange clair, compacte ; surface blanc crème. (Inv. 094.04)

- 180 : amphore africaine cylindrique de grandes dimensions, type Keay LXII, variante tardive ; pâte orange clair, granuleuse ; surface blanc crème, pulvérulente, à l'ext., orange clair à l'int. (Inv. 094.03)

- 181 : amphore africaine cylindrique de grandes dimensions, type Keay LXII, variante tardive. (Inv. 094.08)

- 182 : amphore africaine cylindrique de grandes dimensions, type Keay LXI C ; pâte orange, compacte ; surface 


\begin{tabular}{lll}
1 & 1 & 1 \\
1 & 1 & 1 \\
1 & $j$ & 1 \\
1 & & 1 \\
1 & & 1 \\
1 & & 1 \\
\hline
\end{tabular}


jaune clair à l'ext., orange à l'int., sept incisions sur le sommet du bord. (Inv. 099.02)

- 183 : amphore africaine cylindrique de grandes dimensions, type Keay LXI C ou Bonifay, 1986, fig. 12.55 ; pâte et surface comparables à celles du $n^{\circ} 182$. (Inv. 099.01)

- 184 : amphore africaine cylindrique de grandes dimensions, type Keay LXI A ; pâte rouge brique, granuleuse ; surface chamois, usée. (Inv. 076.02)

- 185: amphore africaine cylindrique de grandes dimensions, type Keay LXI A ; pâte beige orangé, nombreuses incl. de quartz ; surface blanc crème, usée. (Inv. 099.04)

- 186 : amphore africaine cylindrique de grandes dimensions, apparentée au type Keay XXXIV ; pâte rouge brique, compacte ; surface blanc crème, usée à l'ext. (Inv. 076.03) - 187 : amphore africaine cylindrique de grandes dimensions, apparentée au type Keay XXXIV. (Inv. 075.01)

- 188 : amphore africaine cylindrique de grandes dimensions, apparentée au type Keay XXXIV. (Inv. 078.12)

- 189 : amphore africaine cylindrique de grandes dimensions, apparentée au type Keay XXXIV ; pâte brun rouge, compacte ; surface beige-rouge brique. (Inv. 084.06)

- 190 : amphore africaine cylindrique de grandes dimensions, apparentée au type Keay XXXIV ; pâte orange, compacte ; surface à dominante saumon clair. (RouggaBararus, Inv. $\mathrm{n}^{\circ} 229$, exemplaire de comparaison)

- 191 : amphore africaine cylindrique de grandes dimensions, apparentée au type Keay XXXIV. (Inv. 070.07)

- 192 : amphore africaine cylindrique de grandes dimensions, apparentée au type Keay XXXIV. (Inv. 070.09)

- 193 : amphore africaine cylindrique de grandes dimensions, apparentée au type Keay XXXIV (?) ; pâte rouge brique, compacte ; surface saumon à l'ext. (Inv. 071.05)

- 194 : amphore africaine cylindrique de grandes dimensions, apparentée au type Keay XXXIV (?) ; décor peigné. (Inv. 078.14)

- 195: amphore africaine de tradition punique, type Hammamet 1 ; pâte jaune orangé, feuilletée, d'aspect spongieux, nombreuses grosses incl. de quartz ; surface jaune pâle193. (Inv. 127bis.01)

- 196 : amphore africaine de tradition punique, type Hammamet 2 ou 3 ; pâte marron, granuleuse ; surface beige. (Inv. 127bis.03)

- 197 : amphore africaine de tradition punique, type Hammamet 3. (Inv. 078.13)

193. Un exemplaire de comparaison provenant de Pupput possède exactement la même pâte.
- 198 : amphore africaine de tradition punique, type Hammamet 3 ; pâte rouge brique, granuleuse ; surface beige clair. (Inv. 127bis.02)

- 199 : amphore africaine de tradition punique (?) ; décor peigné sur la face ext. du bord. (Inv. 144.01)

- 200 : amphore africaine de tradition punique, type Hammamet 1 ; pâte beige orangé, granuleuse ; surface beige clair. (Pupput, exemplaire de comparaison)

\section{Les ateliers}

La recherche des traces d'ateliers lors de notre prospection s'est heurtée à des problèmes de diagnostic sur le terrain ${ }^{194}$ : de nombreux indices, tels des zones cendreuses, des tessons simplement surcuits (recuits ?) ${ }^{195}$, sont peu éloquents ${ }^{196}$; il nous a semblé préférable de nous en tenir aux preuves indiscutables (déchets de cuisson). Parallèlement, on notera l'absence d'indices en des lieux pourtant connus pour la production de céramique ${ }^{197}$.

\section{- Atelier de Guellala (fig. 15)}

Le site $n^{\circ} 21$, Guellala, sur la rive sud de l'île de Jerba, a livré des témoins peu discutables de la production d'un type d'amphore à anses bifides (nos 201-202) que l'on peut considérer comme une imitation du type Dressel 2/4 ou rapprocher du type Schöne-Mau XXXV, bien connu à Pompéi et à Ostie ${ }^{198}$. Un exemplaire de comparaison ( $\mathbf{n}^{\circ}$ 203) provient d'une tombe de la nécropole de Chamakh ${ }^{199}$. Il y a une grande similitude entre la pâte de ces amphores et celle des productions actuelles des ateliers de Guellala. L'atelier antique de Guellala semble également avoir produit de la céramique commune et culinaire (nos 204-205).

194. Sur les critères de reconnaissance des ateliers, cf. BAl.I.IT P. et al., Artisanat de la céramique, 1991, p. 130-134.

195. Voir par exemple les critiques de M. Mackensen à propos du pseudo atelier de sigillée "africaine "à Classe : MACKk:NS:N M., El Mabrine, 1993, p. 144 sq.

196. Par exemples sur les sites $n^{\circ} 42$ (En Nadour), $n^{\circ} 54$ (Onga), $\mathrm{n}^{\circ} 55$ (Henchir Leich).

197. Par exemple à Raf Raf.

198. PANill. Cl., Anfore, 1973. p. 478-482. Cl. Panella opte pour une origine tripolitaine. (Voir désormais : Fi:Trisss E.. Villas, wine and kilns, 2001].

199. Drix: A., Chammakh, 1992-93 : ID.. Muséographic. 1997. fig. 12. 

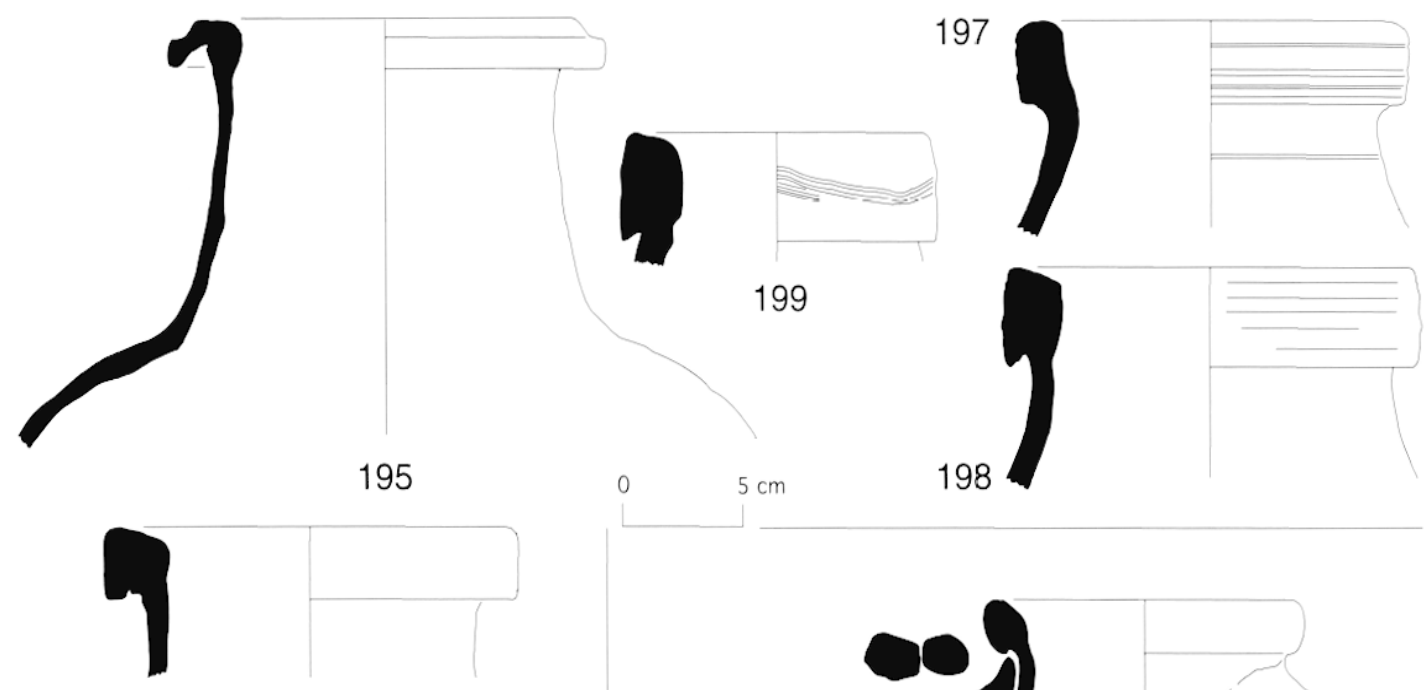

196
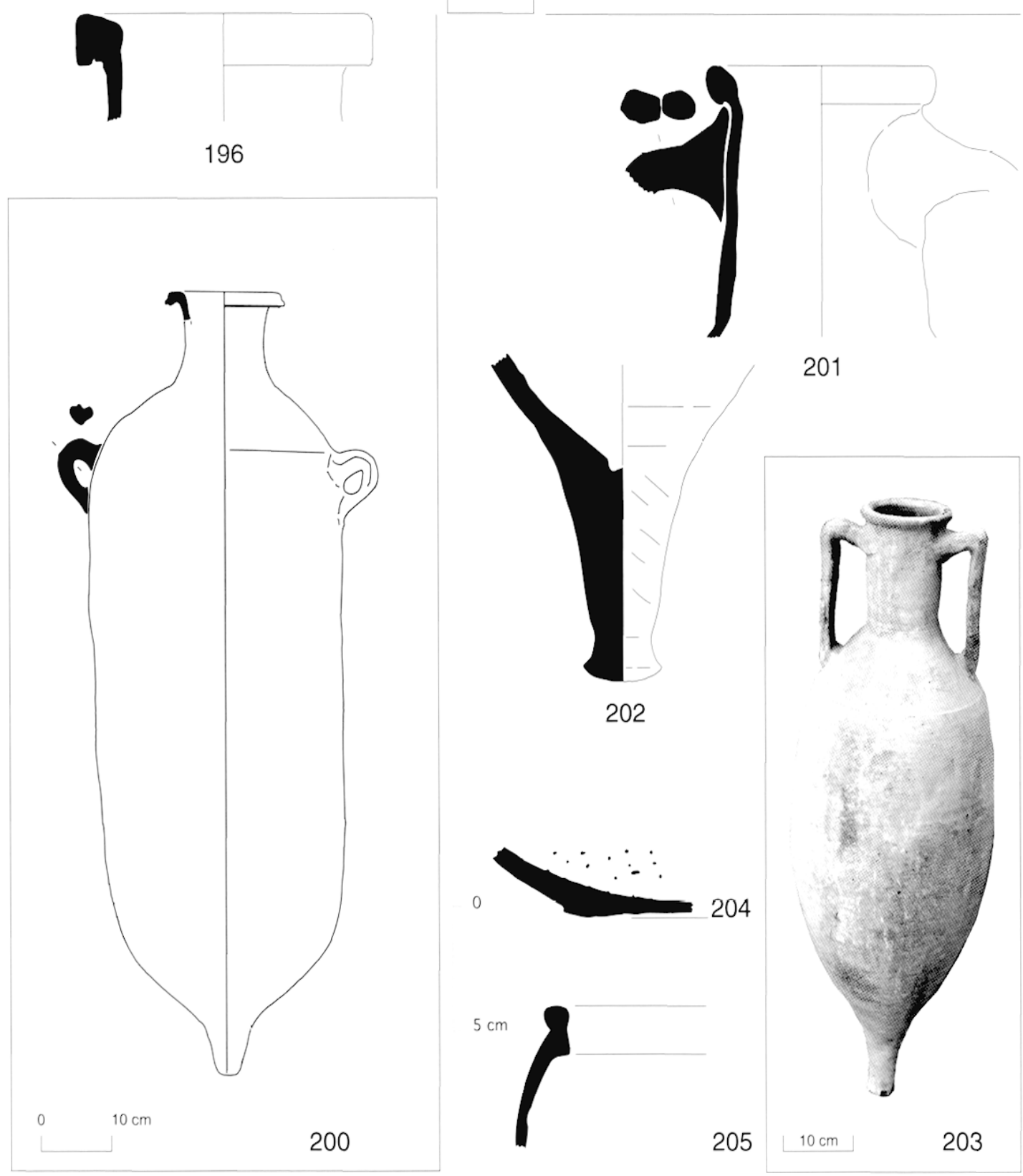

Fig. 15 : Amphores de tradition punique dites du golfe d'Hammamet ( $\left.\mathrm{n}^{\circ \mathrm{s}} 195-199\right)$

( $\mathrm{n}^{\circ} 200$ : exemplaire de comparaison, Pupput) ; atelier (?) de Guellala ( $\mathrm{n}^{\circ \infty}$ 201-202, 204-205) ( ${ }^{\circ} 203$ : exemplaire de comparaison, Chamakh). (M. Bonifay) 


\section{Catalogue}

- 201 : amphore africaine, apparentée au type Dressel 2/4 ou Schöne-Mau XXXV ; pâte orange, sombre, granuleuse, assez nombreux points de chaux, petites incl. de quartz assez clairsemées ; surface blanc crème. (Inv. 021.09)

- 202 : amphore africaine, apparentée au type Dressel 2/4 ou Schöne-Mau XXXV ; pâte et surface comparable à celles du $n^{\circ}$ 201. (Inv. 021.10)

- 203 : amphore africaine, apparentée au type Dressel 2/4 ou Schöne-Mau XXXV. (Musée de Zarzis, provenant de la nécropole de Chamakh, exemplaire de comparaison)

- 204 : céramique commune africaine, mortier à incl. de basalte, tesson surcuit. (Inv. 021.11)

- 205 : céramique culinaire africaine, marmite apparentée au type Hayes 183. (Inv. 021.10)

\section{- Atelier d'Oued el Akarit (fig. 16 et 17)}

Le site $n^{\circ} 39$, Oued el Akarit, est un atelier important qui a produit de la vaisselle culinaire, de la vaisselle commune et des amphores ; il n'y a pas de preuve formelle de la production de sigillée africaine E ( $\mathbf{n}^{\circ} 219$ ) et de lampes Atlante VI ( $\mathbf{n}^{\circ} \mathbf{2 2 0}$ ), pourtant présentes sur le site. La vaisselle culinaire est représentée par les couvercles Hayes 196 ( $\mathbf{n}^{\circ} \mathbf{2 0 6}$ ), Sabratha $104\left(\mathbf{n}^{\circ} \mathbf{2 0 7}\right)$, Hayes $182\left(\mathbf{n}^{\circ} \mathbf{2 0 8}\right)$ et les plats Hayes 181 ( $\mathbf{n}^{\text {os }}$ 209-210). Un " mouton " montre un plat et un couvercle soudés ensemble lors de la cuisson ( $\mathbf{n}^{\circ} \mathbf{2 2 1}$ ). La vaisselle commune comprend des cruches ( $\left.\mathbf{n}^{\circ} \mathbf{2 1 1}\right)$, des mortiers à marli courbe et fond garni de grains de basalte (nos 212-213), des bassins (nos 214-216), des bols (nos 217-218). On relève trois types d'amphores principaux : Africaine I B (nos 222-223), Africaine II D ou Keay XXV.1 (nos 224-225) et Keay XXV.3 ( $\mathbf{n}^{\circ} \mathbf{2 2 6}$ ). Cet atelier semble actif principalement au Ive $\mathrm{s}$.

\section{Catalogue}

- 206 : céramique culinaire africaine, couvercle apparenté au type Hayes 196. (Inv. 039.08)

- 207 : céramique culinaire africaine, couvercle apparenté au type Sabratha 104 ; pâte surcuite de couleur verte avec de nombreuses petites incl. de quartz. (Inv. 039.10)

- 208 : céramique culinaire africaine, couvercle apparenté au type Hayes 182. (Inv. 039.09)

- 209 et 210 : céramique culinaire africaine, plat apparenté au type Hayes 181. (Inv. 039.12 et 07 )

- 211 : céramique commune africaine, cruche. (Inv. $039.15)$
- 212 et 213 : céramique commune africaine, mortiers avec incl. de basalte. (Inv. 039.16 et 17)

- 214 à 216 : céramique commune africaine, bassins à panse carénée. (Inv. 039.20, 19, 18)

- 217 et 218 : céramique commune africaine, jaltes profondes. (Inv. 039.14 et 13)

- 219 : céramique sigillée africaine E, forme Hayes 68 ; pâte orange, compacte ; engobe rouge, mat, int. et ext. (Inv. 039.06)

- 220 : lampe en sigillée africaine de type Atlante VI. (Inv. 039.11)

- 221 : céramique culinaire africaine, mouton entre un plat Hayes 181 et un couvercle apparenté au type Ostia I, fig. 264 ; pâte surcuite de couleur noire avec des incl. de quartz ; surface grésée. (Inv. 039.20)

- 222 et 223 : amphores Africaines I B. (Inv. 039.01 et 02)

- 224 : amphore Africaine II D ou cylindrique de moyennes dimensions, type Keay XXV.1 ; pâte surcuite de couleur grise, compacte, avec des incl. de quartz; surface verdâtre. (Inv. 039.04)

- 225 : idem, raté de cuisson. (Inv. 039.21)

-226 : amphore africaine cylindrique de moyennes dimensions, type Keay XXV.3. (Inv. 039.03)

\section{- Atelier de Thaenae-Thyna (fig. 17)}

Les ateliers sont situés au sud-ouest de la ville ${ }^{200}$, à la fois à l'intérieur et à l'extérieur des remparts. À l'intérieur, on observe de petits tells cendreux avec des tessons surcuits d'amphores Africaines I B (nos 227-228). À l'extérieur, le répertoire de formes est plus vaste : Africaines I, II A " con gradino " (n' 229), II B " con gradino " ( $\mathbf{n}^{\circ} \mathbf{2 3 0}$ ?), II D $\left(\mathbf{n}^{\circ} \mathbf{2 3 1}\right)$, Keay XXV.1 ( $\left.\mathbf{n}^{\circ} \mathbf{2 3 2}\right)$, Keay XXV.2 $\left(\mathbf{n}^{\circ} 233\right.$ ?), Africaine II variante Keay V bis ( $\left.\mathbf{n}^{\circ} 234\right)$. Ces amphores ne sont peut-être pas toutes produites sur place, comme le montrent des exemplaires du type Tripolitaine III et des amphores à fond plat Ostia I, fig. 453 probablement d'origine sicilienne ${ }^{201}$. La pâte de l'atelier de Thaenae est caractérisée par sa teinte rouge sombre et sa granulosité importante, avec très peu de points de chaux.

200. Mme Nabiha Jeddi nous a fait très aimablement visiter ces atelicrs en 1993.

201. Il convient aujourd'hui de remettre en question l'hypothèse d'une production locale de ce type : BEN LAZREG N. et al.. Salsamenta, 1995 , p. 131. 

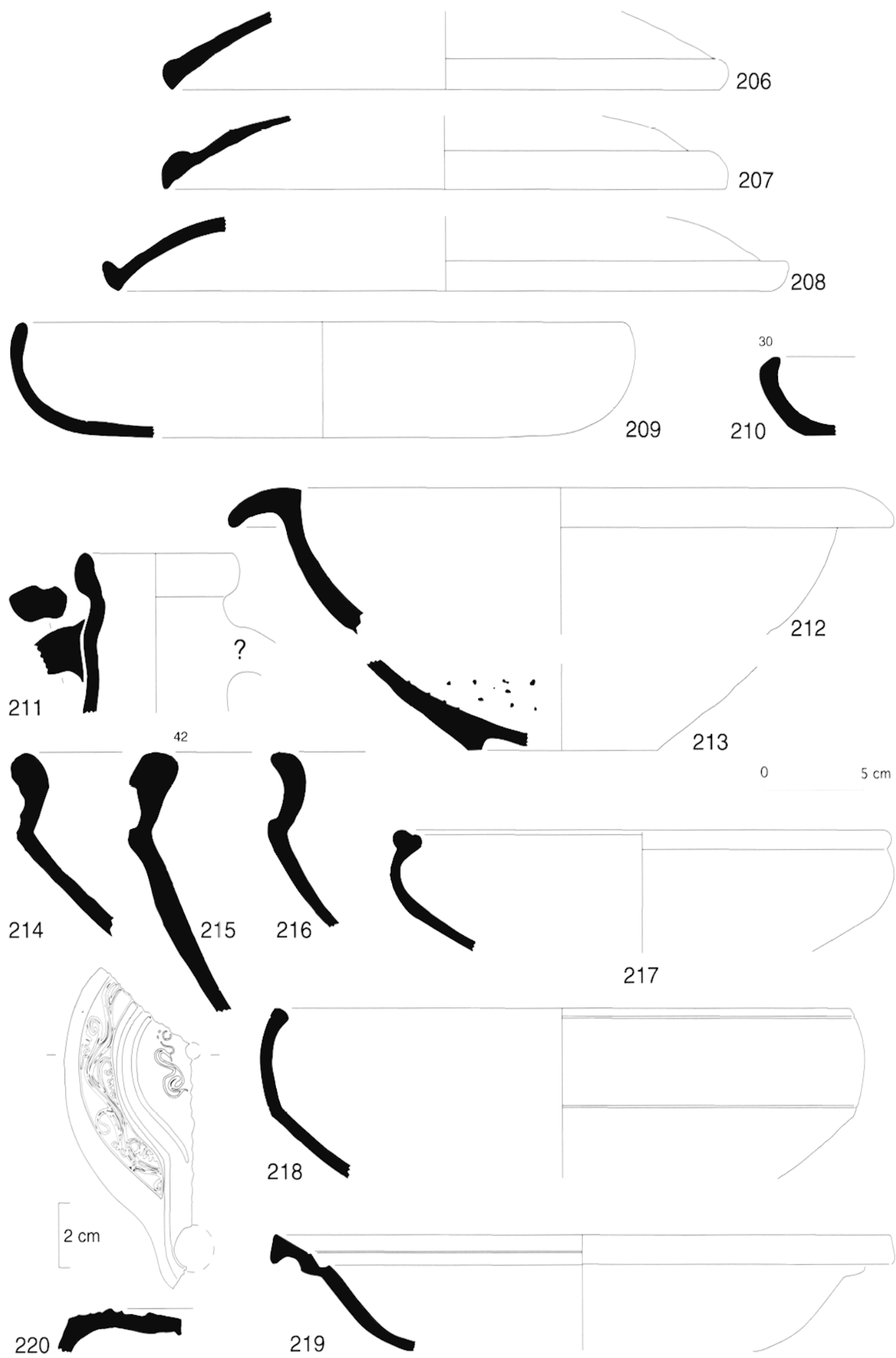

Fig. 16 : Atelier d'Oued el Akarit (les nos 219 et 220 ne sont peut-être pas des productions de l'atelier). (M. Bonifay) 


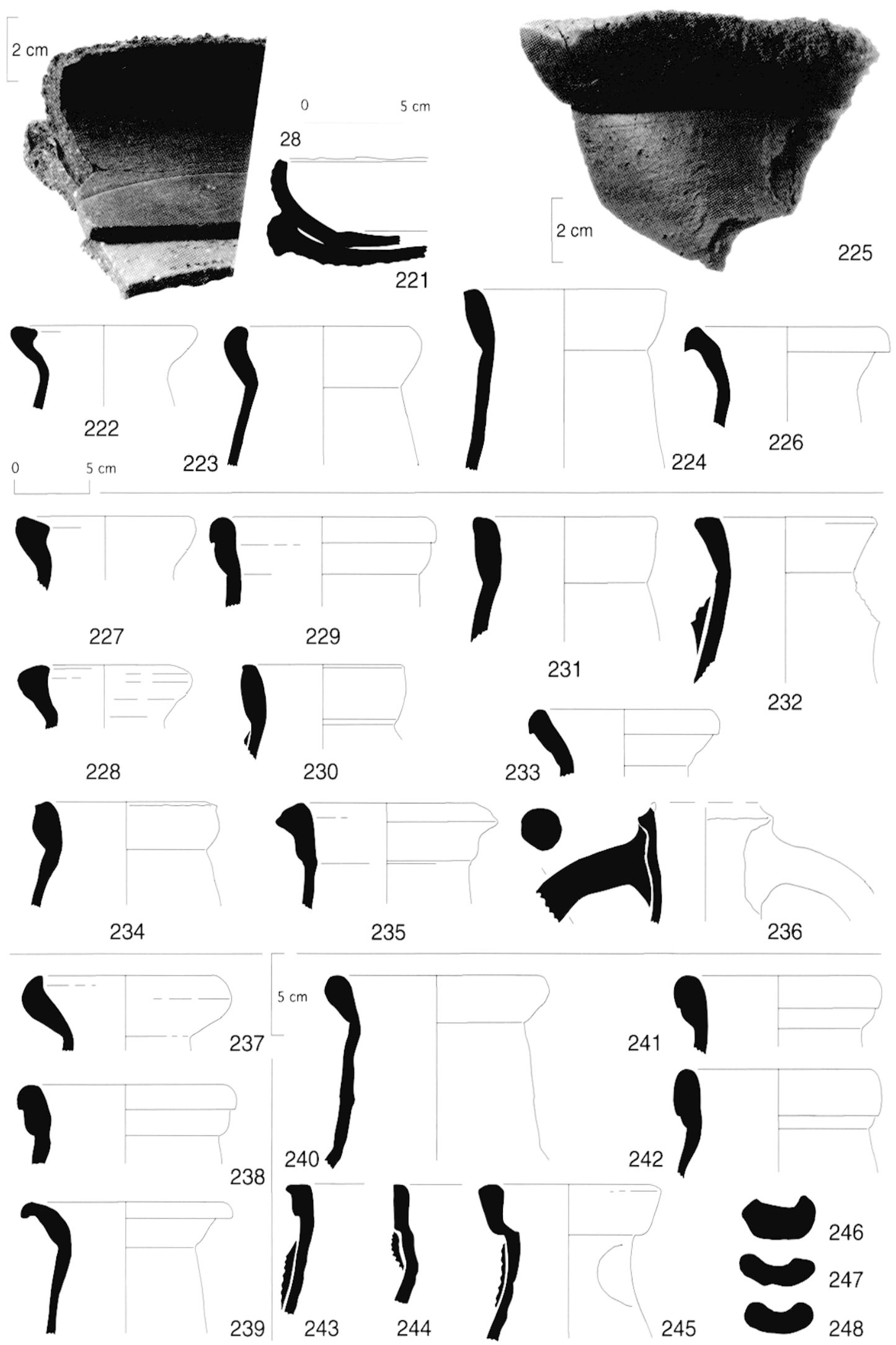

Fig. 17 : Atelier d'Oued el Akarit ( $\left.{ }^{\circ \mathrm{os}} 221-226\right)$; atelier de Thyna-Thaenae ( $\left.\mathrm{n}^{\mathrm{os}} 227-236\right)$; atelier de Salakta-Sullectbum ( ${ }^{\circ \infty}$ 237-239); atelier (?) d'El Assa ( $\left.{ }^{\circ \infty} 240-248\right)$. (M. Bonifay) 


\section{Catalogue}

- 227 : amphore Africaine I B. (Inv. 056.07)

- 228 : amphore Africaine I B ; pâte surcuite de couleur marron, feuilletée, nombreuses petites incl. de quartz; surface grise, usée. (Inv. 056.04)

- 229 : amphore Africaine II A " con gradino "; pâte surcuite de couleur gris-noir, avec des incl. de quartz; surface blanc crème à l'ext. (Inv. 056.03)

- 230 : amphore Africaine II B ou C ; pâte rouge brique, très granuleuse, incl. de quartz, points de chaux ; surface grise à l'ext. (Inv. 056.06)

- 231 : amphore Africaine II D ou cylindrique de moyennes dimensions, type Keay XXV.1. (Inv. 056.01)

- 232 : amphore africaine cylindrique de moyennes dimensions, type Keay XXV.1. (Inv. 056.09)

- 233 : amphore africaine cylindrique de moyennes dimensions, type Keay XXV.2 (?) ; pâte rouge brique, très granuleuse, nombreuses petites incl. de quartz et points de chaux ; surface jaune. (Inv. 056.05)

-234 : amphore Africaine II B, variante Keay V bis. (Inv. 056.08)

- 235: amphore africaine de type Tripolitaine III ; pâte rouge brique, granuleuse, incl. de quartz ; surface beigeblanc à l'ext. (Inv. 056.02)

-236 : amphore sicilienne (?), type Ostia I, fig. 453 (Agora M. 254) ; pâte rouge brique, très granuleuse ; surface surcuite de couleur brune. (Inv. 056.10)

\section{- Atelier de Sullecthum-Salakta (fig. 17)}

Au sein des pâtes africaines, toutes assez semblables, celle de Sullectbum apparait avec netteté202 : très souvent bicolore, rouge brique et gris, elle est caractérisée par une multitude de petits grains blancs de calcaire ${ }^{203}$ associés aux habituelles inclusions translucides (quartz) ; la surface est généralement de couleur grise. Nous ne mentionnons que pour mémoire quelques uns des types d'amphores produits par cet atelier bien connu ${ }^{204}$.

\section{Catalogue}

- 237 : amphore Africaine I A ; pâte orange, constellée de petits grains blancs de calcaire et d'incl. de quartz ; surface blanche. (Inv. 094.02)

- 238 : amphore Africaine II A " con gradino "; pâte rouge orangé, vacuolaire (importantes vacuoles allongées), constellée de petits grains blancs de calcaire et d'incl. de quartz ; surface blanche, usée à l'ext. (Inv. 094.01)

- 239 : amphore africaine cylindrique de moyennes dimensions, type Keay XXV.1/2 ; pâte surcuite de couleur grisnoir, constellée de points de chaux jaunâtres et d'incl. de quartz moins nombreuses et relativement clairsemées ; surface gris verdâtre, grésée. (Inv. 094.10)

\section{- Atelier d'El Assa (fig. 17)}

Les preuves de la présence d'un atelier de céramique sur le site $\mathrm{n}^{\circ} 166$, El Assa, sur la face occidentale du Cap Bon, sont assez ténues : à l'extrémité nord du site, un épandage serré de tessons d'amphores dans un terrain cendreux, à proximité de mottes d'argile mal cuite. Beaucoup de fragments appartiennent à des amphores africaines de type classique : notamment des Africaines II A et II B " con gradino " (nos 241-242). Mais le plus surprenant est de recueillir des bords (nos 244-245) et des anses (nos 246-248) qui évoquent le type Dressel $30^{205}$.

\section{Catalogue}

- 240 : amphore Africaine II (?), variante. (Inv. 166.08)

- 241 : amphore Africaine II A " con gradino "; pâte surcuite de couleur brune orangée, nombreux points de chaux, incl. de quartz; surface beige à l'ext. (Inv. 166.07)

- 242 : amphore Africaine II A " con gradino ". (Inv. 166.09) - 243 : amphore Africaine II (?), variante ; pâte orange, granuleuse ; surface beige clair à l'ext. (Inv. 166.10)

- 244 et 245 : amphores africaines apparentées au type Dressel 30. (Inv. 166.06 et 02)

- 246 à 248 : anses d'amphores africaines apparentées au type Dressel 30. (Inv. 166.03 à 05)

205. Le type Dressel 30 de fabrication tunisienne est connu à Nabeul dans divers contextes échelonnés entre le IIIC et le IV siècles. [Voir désormais : GHALIA T., BONIIAY M., CAPHLLI.I Cl., Sidi Zahruni, à paraître : atelier de Sidi Aoun]. 


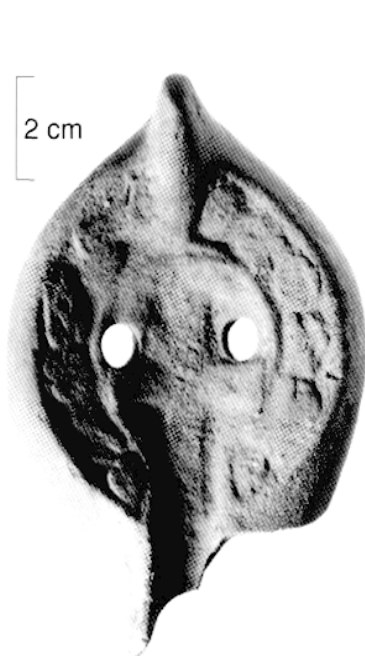

253
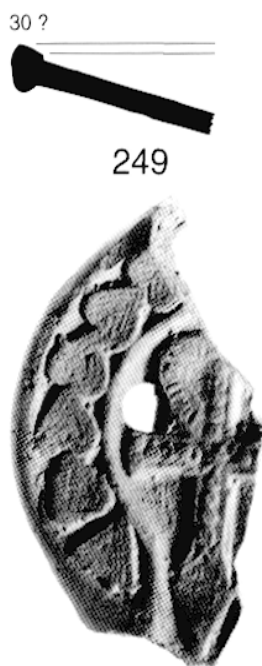

254
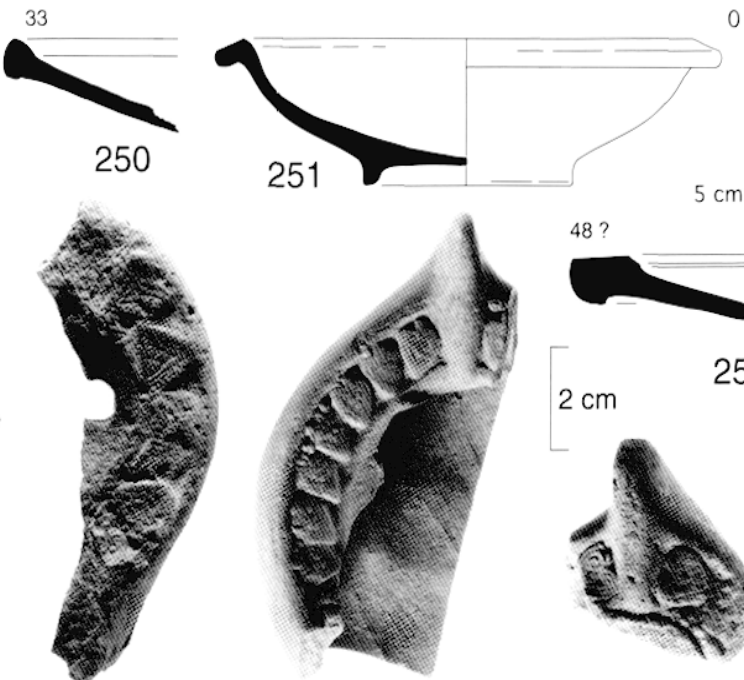

255

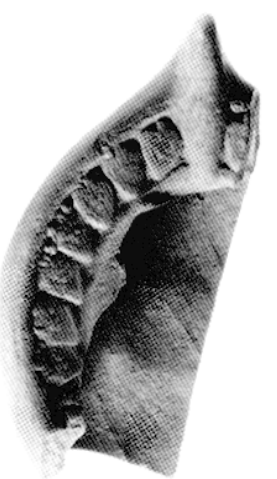

256

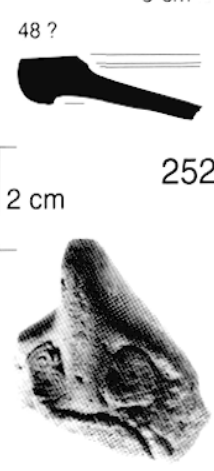

257

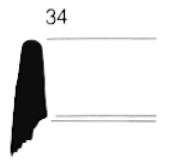

258

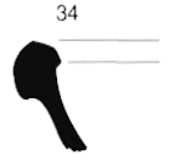

260
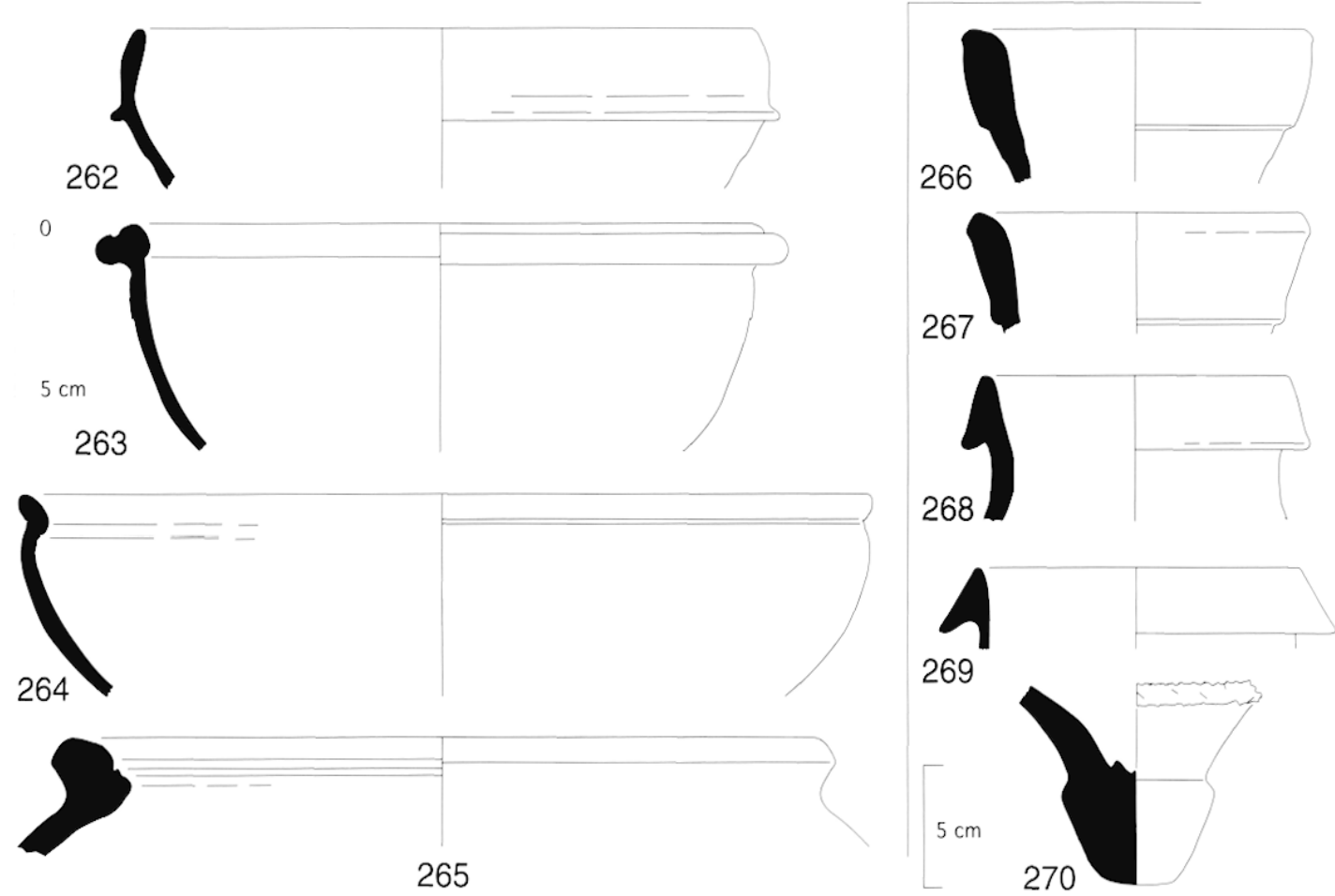

Fig. 18 : Site nos $85-86$ (Jezira). (M. Bonifay) 


\section{Contextes homogènes}

- Un site de l'Antiquité tardive : Jezira (sites $n^{\text {os }} 85$ et 86) (fig. 18)

La céramique recueillie sur ce site est homogène et révèle une occupation restreinte à l'Antiquité tardive : quelques éléments peuvent remonter à la seconde moitié du ve $s$. (Hayes 87, 12/102) mais la majorité appartiennent à l'époque byzantine tardive. En effet, il convient d'attribuer au vile $s$. les formes de sigillée africaine Hayes 105 (nos 249-250), 108 ( $\mathbf{n}^{\circ} \mathbf{2 5 1}$ ) et Atlante XLI, 3-4 ( $\mathbf{n}^{\circ} \mathbf{2 5 2}$ ), les lampes Atlante X à décor surmoulé (nos $253-257$ ), certaines céramiques culinaires, comme la marmite dérivée de la forme Hayes $23\left(\mathbf{n}^{\circ} \mathbf{2 5 8}\right)$, et des céramiques communes : plat imité de Hayes 103 ( $\mathbf{n}^{\circ}$ 259) et bassins à bord épaissi souligné par une rainure interne (nos 260-261). Le bol à listel $n^{\circ} 262$ est proche des formes connues à Carthage à la même époque $^{206}$; la jarre $\mathbf{n}^{\circ} \mathbf{2 6 5}$ est connue sur plusieurs sites de Tunisie occupés au viI ${ }^{\mathrm{e}} \mathrm{s}^{207}$. Parmi les amphores on remarque une forme $\left(\mathbf{n}^{\circ} \mathbf{2 6 6}\right)$ très bien attestée dans les niveaux de la fin du $\mathrm{VI}^{\mathrm{e}}$ et de la première moitié du $\mathrm{VII}^{e} \mathrm{~s}$. à Marseille ${ }^{208}$ et qui est produite, au moins en partie, sur l'atelier d'Henchir ech Chekaf209. On ne sait si on doit rattacher les fragments d'amphore Keay XXXIV (nos 269-270) à cette période d'occupation ou bien à celle de la deuxième moitié du ve s.

\section{Catalogue}

- 249 : céramique sigillée africaine D, forme Hayes 105 ; pâte rouge brique, compacte, légèrement vacuolaire, nombreux gros points de chaux, petites incl. de quartz ; engobe orange, usé à l'int. (Inv. 085.07)

- 250 : céramique sigillée africaine $D$, forme Hayes 105 ; pâte orange, compacte ; engobe orange, satiné à l'int., écaillé sur le bord, coulures à l'ext. (Inv. 085.08)

206. HAYES J.W., Pottery report, 1978, p. 69 : * Flanged bowls, class II imitation *; FLt.FORD M. et PEACOCK D.P.S., Avenue Bourguiba. 1984, p. 202 : formes 15 et 16.

207. Carthage : HAYES J.W., Pottery report, 1978, fig. 17, n"s 62-63 ; Sidi Jdidi : BEN ABED A., BoNifaY M. et Fix(T M., Note préliminaire, 1997, fig. $4, n^{\circ} 26$ et fig. 10, $n^{\circ 5} 57-58$; atelier d'Henchir ech Chekaf : PEACOCK D.P.S., Bl:N LAZREG; N. et BEIAOC' F., Roman Amphora Production, 1989, fig. $8, \mathrm{n}^{\circ} 16$.

208. BONIFAY M. et PIERI D., Amphores, 1995, fig. $4, \mathrm{n}^{\circ} 32$.

209. PBACOCK D.P.S., BEN LAZREG N. et BEIAOII F., Roman Amphora Production, 1989, fig. $8, n^{\circ} 9$.
- 251 : céramique sigillée africaine $D$, forme Hayes 108 ; pâte orange, très granuleuse ; engobe orange, lustré, usé à l'int. (Inv. 085.09)

- 252 : céramique sigillée africaine $\mathrm{D}$, apparentée à la forme Hayes 105/106 variante ; pâte orange, compacte ; engobe orange, mat à l'int., coulures à l'ext. (Inv. 085.18)

- 253 : lampe en sigillée africaine, type Atlante X (Hayes II $B$ ) ; bandeau décoré de motifs très empâtés (colombes, disques, feuilles cordiformes...), sur le disque croix monogrammatique bouclée à gauche; pâte orange, très compacte ; engobe rouge, écaillé. (Inv. 085.01)

- 254 : lampe en sigillée africaine, type Atlante X (Hayes II B) ; bandeau décoré de motifs empâtés ( 5 feuilles cordiformes pointes tournées vers l'anse, 1 triangle à la jonction avec le bec), sur le disque croix monogrammatique à décor ocellé, bouclée à gauche; pâte comparable à celle du $\mathrm{n}^{\circ} 253$, avec quelques gros points de chaux; engobe orange clair, mat. (Inv. 085.04)

- 255 : lampe en sigillée africaine, type Atlante X (Hayes II B) ; bandeau décoré de motifs empâtés (feuilles cordiformes, triangle et lièvre courant à gauche) ; pâte orange, très granuleuse ; engobe orange clair, mat. (Inv. 085.03)

- 256 : lampe en sigillée africaine, type Atlante X (Hayes II B) ; bandeau décoré de petites feuilles cordiformes ; pâte orange, très compacte, très petites incl. de quartz et brunes ; engobe rouge, écaillé. (Inv. 085.02)

- 257 : lampe en sigillée africaine, type Atlante X (Hayes II B) ; bandeau décoré de feuilles cordiformes empâtées ; pâte orange, très granuleuse ; engobe orange clair, mat. (Inv. 085.05)

- 258 : céramique culinaire africaine, forme dérivée de Hayes 23 (?) ; pâte rouge brique, feuilletée, incl. de quartz ; engobe noir à l'int., coulures à l'ext. (Inv. 085.19)

- 259 : céramique commune africaine, forme dérivée de Hayes 103 (?) ; pâte orange, granuleuse, compacte ; surface grossièrement lissée (rayures obliques) à l'int. (Inv. 085.10) - 260 : céramique commune africaine, hassin à bord renflé ; pâte orange à cœur gris, granuleuse, grosses incl. de quartz ; surface orange, ext. du bord blanc crème. (Inv. 085.17)

- 261 : céramique commune africaine, bassin à bord renflé ; pâte rouge brique, granuleuse ; surface orange, usée. (Inv. 086.01)

- 262 : céramique commune africaine, bol à listel apparenté à la forme Fulford 1984, fig. $77, n^{\circ} 14$; pâte brune, très compacte, très petites incl. de quartz clairsemées ; surface brune, beige clair au-dessus du listel. (Inv. 085.13) 

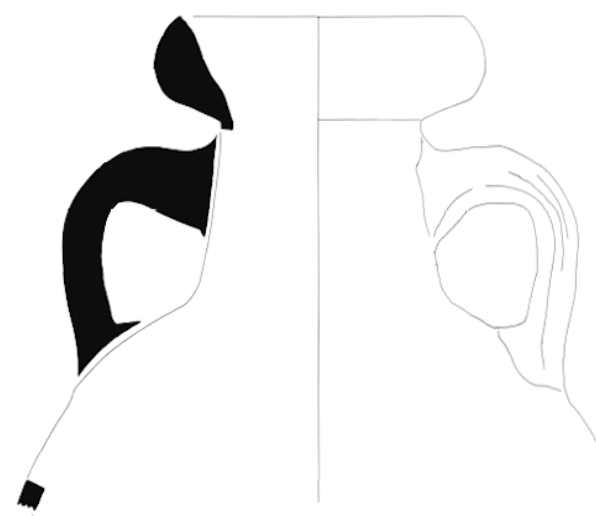

271

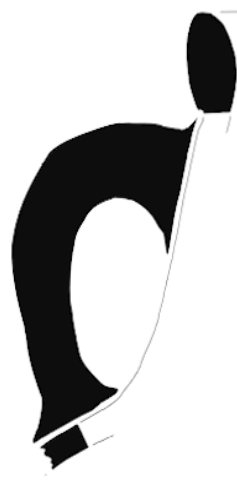

273

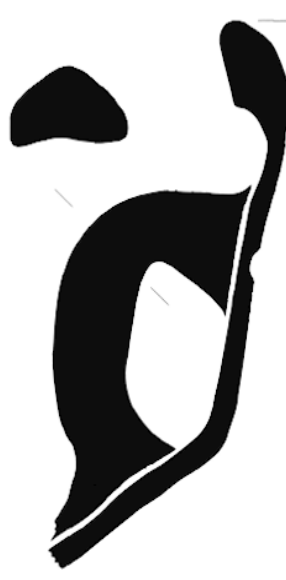

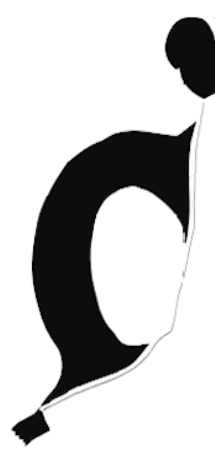

272
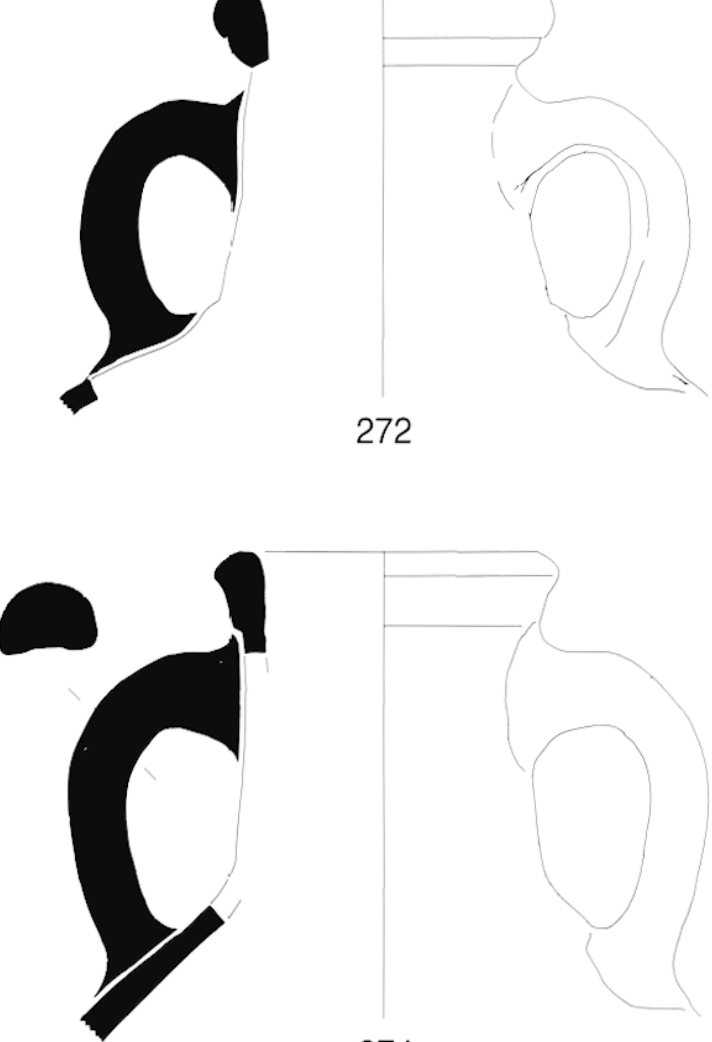

274
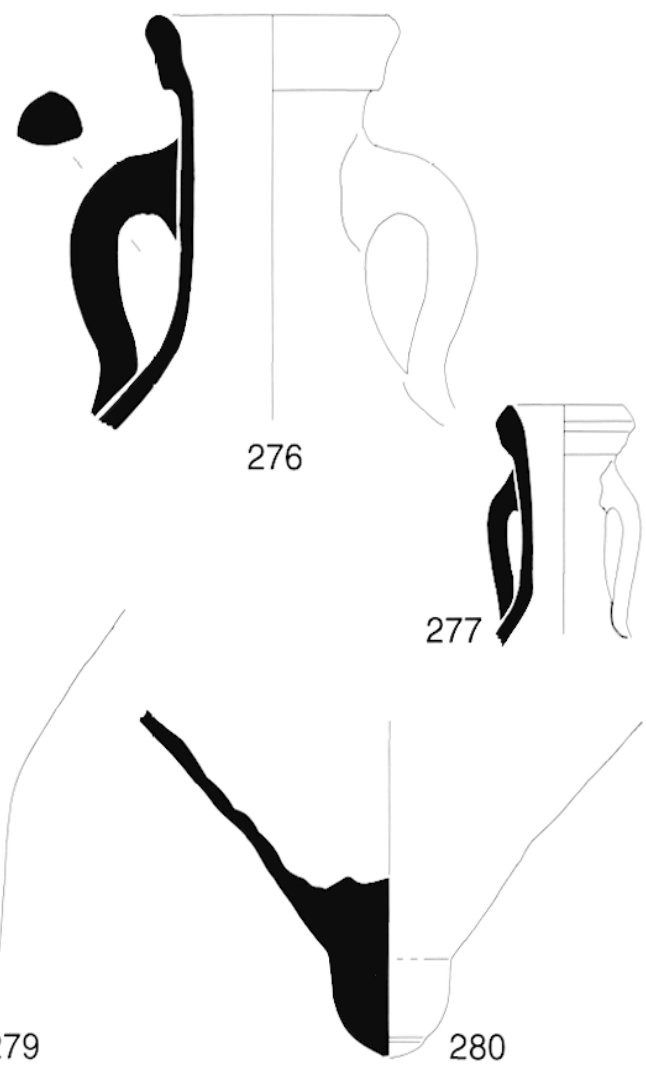

280

Fig. 19 : Site $n^{\circ} 94$ (Salakta-Sullecthum) : objets récupérés en mer, amphores africaines. (M. Bonifay) 
- 263 : céramique commune africaine, apparentée à un bol à listel ; pâte orange, granuleuse ; surface orange. (Inv. 085.11)

- 264 : céramique commune africaine, jatte profonde ; pâte rouge brique, très granuleuse, incl. de quartz; surface beige orangé à l'ext. (Inv. 085.12)

- 265 : céramique commune africaine, jarre ; pâte jaune orangé, granuleuse ; surface jaune clair à l'ext., orange à l'int. (Inv. 085.20)

- 266 : amphore africaine cylindrique de grandes dimensions, forme Bonifay 1986, fig. 12, $\mathrm{n}^{\circ} 55$; pâte rouge brique, compacte ; surface jaune clair à l'ext., brune orangée à l'int. (Inv. 085.23)

- 267 : amphore africaine cylindrique de grandes dimensions (?), apparentée au type Keay LXI ; pâte orange, très granuleuse, nombreuses incl. de quartz; surface beige jaunâtre, pulvérulente à l'ext., orange à l'int. (Inv. 085.22)

- 268: amphore africaine cylindrique de grandes dimensions, apparentée au type Keay LX (?) ; pâte orange, granuleuse, nombreuses incl. de quartz; surface beige à l'ext. (Inv. 085.21)

- 269 : amphore africaine cylindrique de grandes dimensions, apparentée au type Keay XXXIV ; pâte rouge brique, feuilletée, incl. de quartz; surface beige saumon. (Inv. 085.25)

- 270 : amphore africaine cylindrique de grandes dimensions, fond apparenté au type Keay XXXIV. (Inv. 085.24)

\section{- Le port de Sullecthum-Salakta : objets récupérés en mer (fig. 19 et 204)}

Le matériel récupéré en mer lors d'une plongée effectuée en 1992 dans la baie de Salakta est constitué de nombreux fragments d'amphores qui apportent quelques informations sur le commerce drainé par la ville durant l'antiquité. Au III ${ }^{e} s$., on constate le départ de denrées produites à Salakta : huile dans les Africaines I A ( $\left.\mathbf{n}^{\circ} \mathbf{2 7 1}\right)$ et salaisons de poissons (?) dans les Africaines II A " con gradino " $\left(\mathbf{n}^{\circ} \mathbf{2 7 2}\right)$; des marchandises semblent arriver d'autres villes d'Afrique (nos 273-274). Pour l'Antiquité tardive, les produits de l'arrière-pays de Salakta sont toujours présents ( $\mathbf{n}^{\circ} 275$ : amphore Keay LXI C, fin $\mathrm{VI}^{\mathrm{e}}-\mathrm{VII}^{\mathrm{e}} \mathrm{s}$.) mais les importations prennent une part importante : six cols d'amphores LRA $1 B 1^{210}\left(\mathbf{n}^{\circ} \mathbf{2 8 1}\right)$, probablement importées de Cilicie, présentent le même dessin de concrétions marines et laissent supposer qu'elles ont été trouvées groupées, peut-être à l'emplacement d'une épave : un col d'amphore LRA $2 \mathrm{C}\left(\mathbf{n}^{\circ} \mathbf{2 8 2}\right)$ indique que les relations avec l'Orient subsistent au VII ${ }^{\mathrm{e}}$ siècle ${ }^{211}$.

\section{Catalogue}

- 271 : amphore Africaine I A ; pâte brun-rouge, constellée de grains de calcaire et de quartz (probablement atelier de Salakta) ; surface orange, rugueuse (érodée par la mer), percée par quelques grains blancs. (Inv. 094.11)

- 272 : amphore Africaine II A " con gradino "; pâte bicolore orange et grise (vers la surface), constellée de grains de calcaire et de quartz (probablement atelier de Salakta) ; surface brune orangée. (Inv. 094.13)

- 273 : amphore Africaine II B ou C; pâte rouge brique, feuilletée, incl. de quartz clairsemées, quelques points de chaux ; surface brune orangée. (Inv. 094.14)

- 274 : amphore africaine, apparentée au type Uzita pl. 52, $\mathrm{n}^{\circ} 10$; pâte grise (décolorée par la vase ?), vacuolaire, rares incl. de quartz; surface grise, rugueuse ; int. entièrement poissé. (Inv. 094.20)

- 275 : amphore africaine cylindrique de grandes dimensions, type Keay LXI C ; pâte brune (décolorée par la vase ?), compacte, feuilletée, peu d'incl., quelques grains de chaux ; surface brune. (Inv. 094.21)

- 276: amphore africaine apparentée au type spatbeion (?) ; pâte gris-noir au cœur (décolorée ?), orange en surface, petites incl. de quartz; surface marron-beige ; int. poissé. (Inv. 094.23)

- 277 : amphore africaine, spatbeion de petites dimensions ; pâte gris-noir au cœur (décolorée ?), orange en surface, granuleuse ; surface chamois. (Inv. 094.16)

- 278 : fond d'amphore Africaine I (?) ; pâte brune au coeur: grise en surface (décolorée?), nombreuses petites incl. de quartz; surface beige ; traces de poix sur le fond. (Inv. 094.12)

- 279 : fond d'amphore de type Africaine II (?) ou Keay XXV.1 (?) ; pâte rouge brique, constellée de grains de calcaire et de quartz (probablement atelier de Salakta); surface brune orangée ; int. poissé. (Inv. 094.15)

-280 : fond d'amphore africaine cylindrique de grandes dimensions (type Keay LV, LVI ou LVII ?) ; pâte rouge brique, petites incl. de quartz, quelques gros points de chaux ; surface grise à l'ext. ; traces de poix à l'int. (Inv. 094.17) 

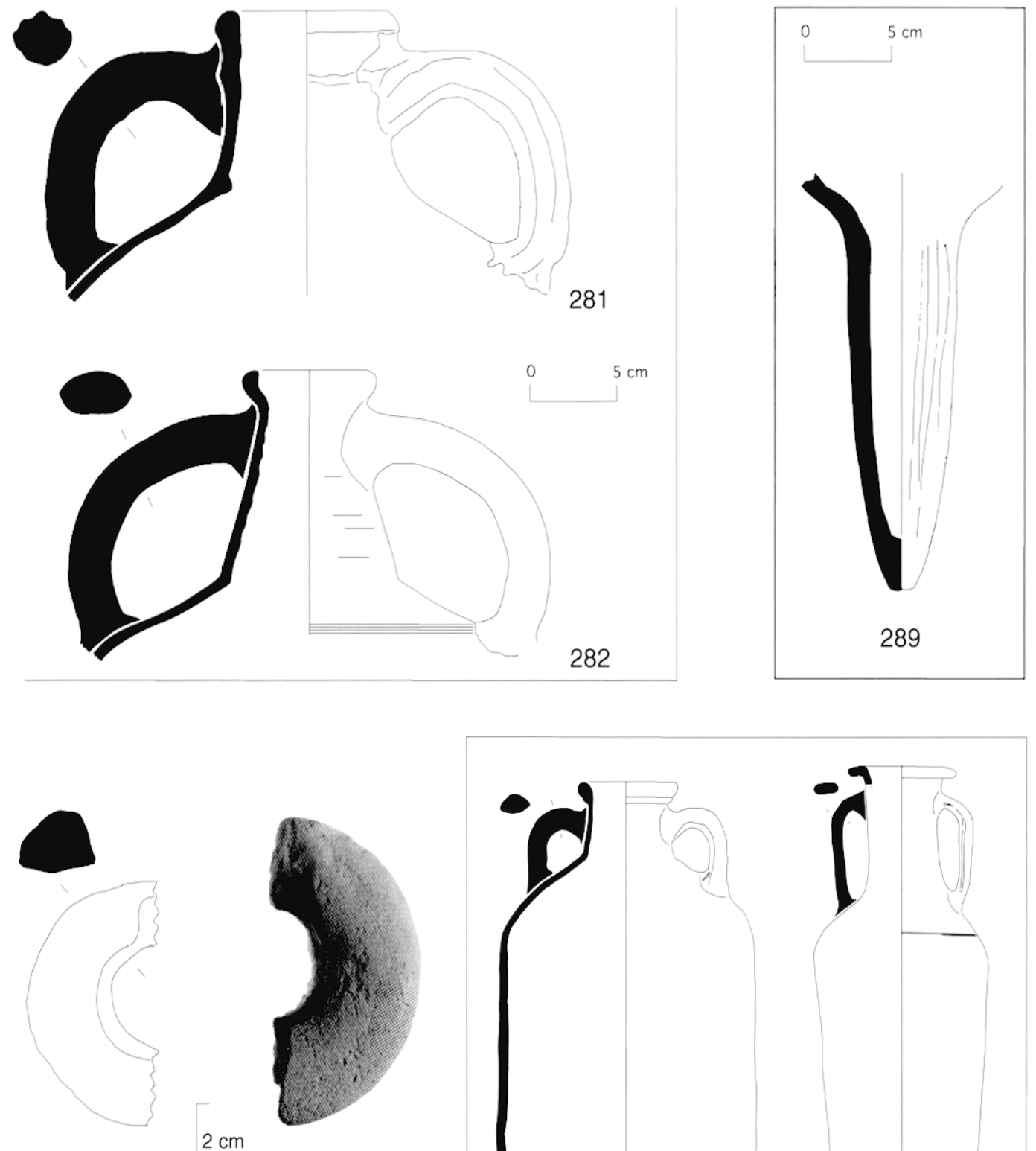

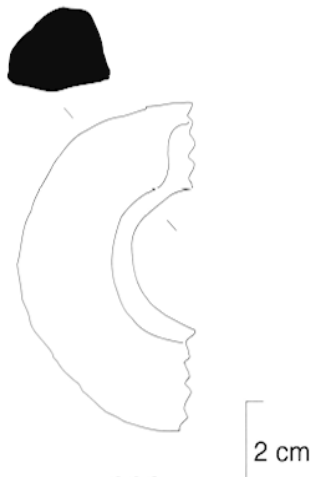

283

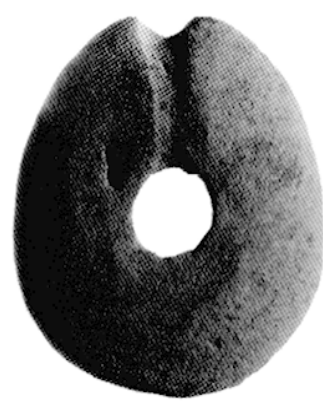

285
284

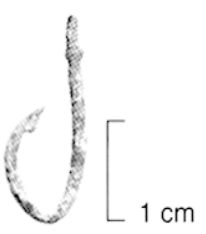

286

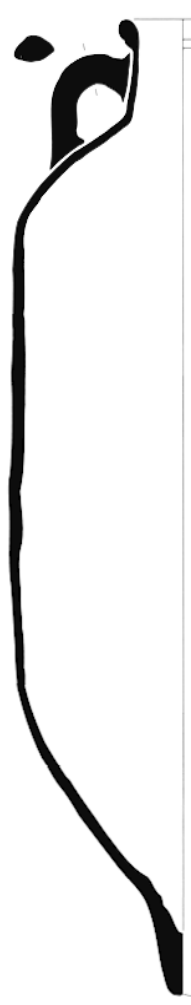

287

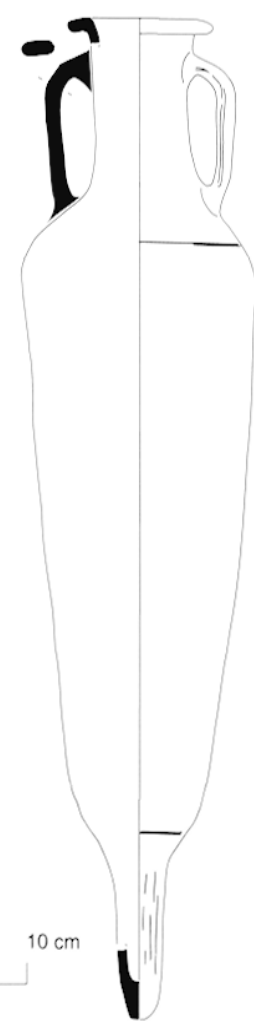

288

Fig. 20 : Site $n^{\circ} 94$ (Salakta-Sullecthum) : objets récupérés en mer, amphores orientales ( ${ }^{\circ \%}$ 281-282); témoins des activités halieutiques : pesons de filets ( ${ }^{\circ}$ 283-285), hameçon en bronze $\left(\mathrm{n}^{\circ} 286\right)$; amphores destinées au transport des salaisons de poissons (?) : Nabeul-Neapolis ( $\mathrm{n}^{\circ} 287$ ), Salakta-Sullecthum ( ${ }^{\circ}$ 288), El Jem-Thysdrus, Maison d'Africa $\left(\mathrm{n}^{\circ}\right.$ 289). (M. Bonifay ; photographies Chr. Durand) 
- 281 : amphore orientale, type LRA 1B ; pâte grise (décolorée?), assez fine, d'aspect sableux, incl. blanches et petits points rouges; surface beige, rugueuse; traces de poix à l'int. (Inv. 094.19)

- 282 : amphore orientale, type LRA $2 \mathrm{C}$; pâte grise (décolorée ?), assez fine, d'aspect sableux, incl. blanches et paillettes de mica blanc; surface beige ; int. poissé. (Inv. 094.18)

\section{C - Faciès régionaux}

L'examen de la diffusion des différentes catégories de céramique (fig. 21 et 206) permet, semble-t-il, de distinguer cinq grandes zones géographiques. La zone de Carthage, bien connue, est laissée de côté.

\section{a) Le golfe de Gabès (Tripolitaine nord-occiden- tale)}

Cette région, tout au long de l'époque romaine, est le terrain de prédilection des amphores tripolitaines (type Schöne-Mau XXXV, Tripolitaines I et III) dont on connaît au moins les ateliers de Guellala (Jerba) et de Zitha (près de Zarzis). Bien alimentée par les importations italiques et gauloises (y compris la sigillée luisante du début du ve $s$.), elle est surtout caractérisée, à partir du IVe $s$., par la présence massive des productions du Sud tunisien (sigillée E et ateliers de l'intérieur) ; les autres catégories de sigillée africaine (sigillées A, D et surtout les ateliers de Tunisie centrale : C) sont néanmoins bien attestées. Cette région tire encore son originalité des lampes en sigillée Atlante I-IV, VI, VIII B et des lampes tripolitaines. Le matériel publié récemment à Sabratha ${ }^{212}$ est un bon élément de comparaison, même si, au contact de la Byzacène méridionale, certains ateliers (par exemple Oued el Akarit) produisent déjà des formes d'amphores plus spécifiques à cette dernière région.

\section{b) Le sahel (Byzacène méridionale)}

Jalonnée de villes importantes (Thaenae, Sullecthum, Leptiminus, Hadrumetum) connues comme autant d'ateliers par l'épigraphie des timbres d'amphores, cette région est, de la fin du $\mathrm{II}^{\mathrm{e}} \mathrm{s}$. au

212. DORE J. et KEAY N., Sabratha, 1989) ; F(LFORI M. et TOMBER R., Sabratha, 1994. milieu du ve s., le lieu de production par excellence des amphores africaines classiques (Africaines I et II) et des amphores cylindriques de moyennes dimensions (Keay XXV) ; elle garde, de ce point de vue, une importance encore considérable aux $\mathrm{VI}^{\mathrm{e}}-\mathrm{VII}{ }^{\mathrm{e}} \mathrm{s}$. avec les types Keay LXI-LXII, fabriqués dans l'arrière-pays de Salakta et largement exportés, et le type Keay XXXIV, à diffusion plus locale dans la région d'El Jem Acbolla. La vaisselle est alimentée massivement, à partir du III $\mathrm{s}$., par les ateliers du centre de la Tunisie (sigillée $\mathrm{A} / \mathrm{D}$ et $\mathrm{C}$ ) et ce jusqu'à la fin du ve $\mathrm{s}$. (sigillée C5). Ce qui, du point de vue de la vaisselle, se passe aux $\mathrm{VI}^{\mathrm{e}}-\mathrm{VII}^{\mathrm{e}} \mathrm{s}$. est moins clair. Certains ateliers (un peut-être à proximité de Leptiminus ?) fabriquent des formes de sigillée D (Hayes 105), d'autres peut-être (plus au sud ?) des formes dérivées de la sigillée C5 (Hayes $90 \mathrm{~B}$ ). Les lampes en sigillée sont issues des ateliers de Tunisie centrale (type Hayes II A) peut-être jusqu'à une date tardive de l'époque byzantine. La céramique commune dont on commence à bien connaître le faciès tardif présente elle aussi une originalité très nette par rapport au faciès mieux connu de Carthage (types nos 112-117, 118-119, 121-125).

\section{c) Le golfe d'Hammamet (Byzacène septentrio- nale)}

On ne sait rien sur la production éventuelle d'amphores africaines classiques entre Hadrumetum et Neapolis - deux villes où elle est en revanche attestée. Un groupe d'amphores paraît néanmoins caractéristique du golfe d'Hammamet : ce sont des amphores de tradition punique à anses sur l'épaulement dont on suit l'évolution en trois types principaux du II ${ }^{\mathrm{e}}$ au VII ${ }^{\mathrm{e}}$ s. (nos 195-197). Du point de vue de la vaisselle, normalement alimentée par les sigillées gauloises, puis par la sigillée africaine A et surtout $C$, cette région affirmera son originalité avec la diffusion, aux ve et $\mathrm{VI}^{\mathrm{e}} \mathrm{s}$., des produits de l'atelier de Sidi Khalifa (Hayes 61 B, 88, 103).

\section{d) Le Cap Bon}

On savait que Neapolis avait produit des amphores africaines II C (Panella 1982, p. 175) ; on serait tenté aujourd'hui d'ajouter le type II A " con gradino "213 mais on connaît surtout maintenant un important

213. Bien représenté sur le site de l'usine de salaison, voir infra. 


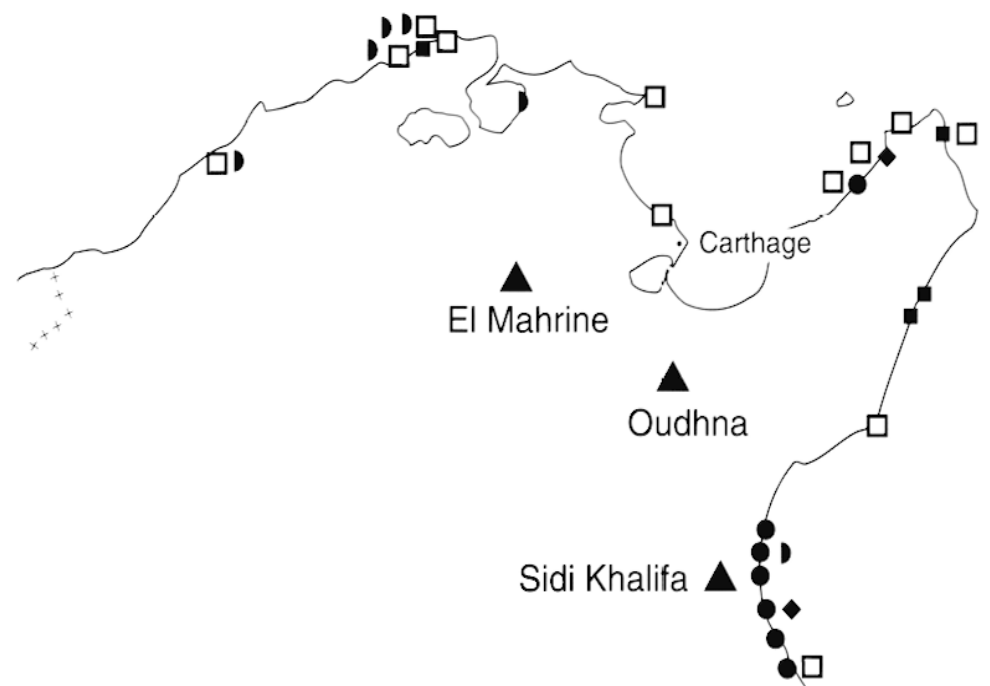

D

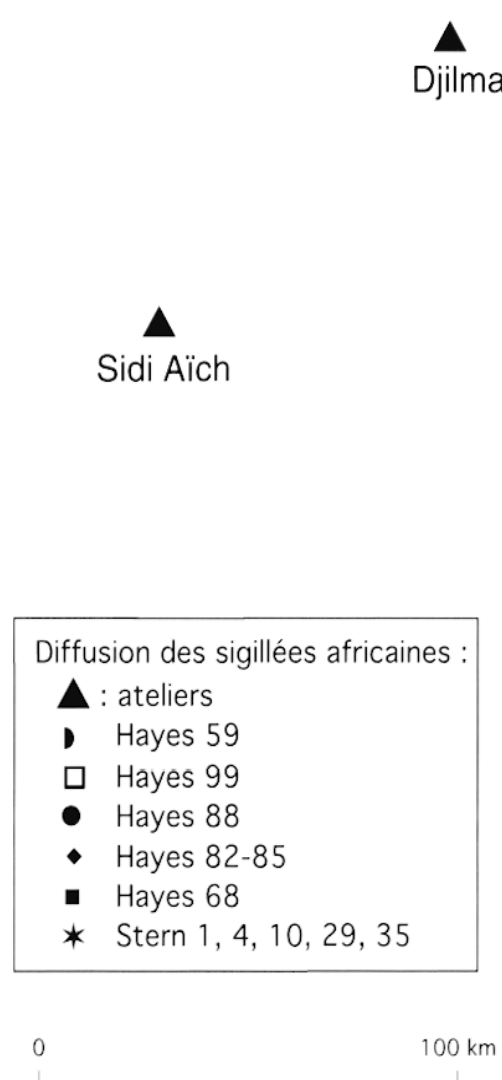

Sidi Marzouk

Tounsi

Fig. 21 : Production et diffusion de quelques formes de sigillée africaines sur le littoral tunisien. (M. Bonifay) 
atelier à $6 \mathrm{~km}$ de la ville qui a produit de nombreux types plus tardifs et amplement diffusés : Keay XXV.2, Keay XXXV B, Keay LV A. La présence de casettes de cuisson tant sur cet atelier que sur le site même de Neapolis fait soupçonner la présence, à proximité de cette ville, d'un atelier de sigillée. Mais, en dehors des importations assez abondantes de sigillée italique puis de sigillée africaine A et $\mathrm{C}$, ce sont surtout les produits de l'atelier d'Oudhna (forme Hayes 99) qui apparaissent avec constance sur les sites littoraux du Cap Bon. Cette région tire également son originalité de la production (marginale ?) d'amphores apparentées au type Dressel 30 et par l'importation fréquente de céramiques modelées de Pantelleria.

\section{e) La côte nord}

Le faciès céramique de cette région n'apparaît pas avec netteté. On relève simplement l'ample diffusion des sigillées africaines D de l'atelier d'El Mahrine (Hayes $61 \mathrm{~A}, 67,76$ ) aux côté d'amphores dont la production est attestée dans le Cap Bon (Africaines II C, Keay XXXV B). La seule originalité provient de la proportion, apparemment plus élevée qu'ailleurs, de céramiques modelées.

\section{3 - La céramique, témoin des activités écono- miques}

Parmi les activités économiques liées à la mer dont la céramique peut se faire le témoin, la première est sans aucun doute le commerce. Dans une province qui, en raison de ses capacités productives, a toujours eu tendance à l'auto-consommation, les importations d'amphores et de vaisselle revêtent une importance particulière. On doit toutefois observer qu'aucune partie du littoral ne se distingue par une forte proportion de céramiques importées et qu'à l'inverse, aucune région de l'Empire n'est particulièrement bien représentée. De l'Espagne, on remarque ${ }^{214}$ des amphores Dressel 20 antérieures au développement massif de l'oléiculture en Afrique et quelques amphores tardives à salaison (Almagro 50 et $51 \mathrm{~A}-\mathrm{B}$ ). La Gaule exporte sa vaisselle au $\mathrm{I}^{\text {er }} \mathrm{s}$. et son vin au $\mathrm{II}^{\mathrm{e}} \mathrm{s}{ }^{215}$; de manière plus

214. À Nabeul, remployées dans l'usine de salaison.

215. Amphore gauloise 4 dans la nécropole de Pupput. étonnante, on va retrouver les traces d'un nouveau flux commercial vers le ve $\mathrm{s}$. avec les sigillées luisantes en provenance de Savoie : ces produits (qui accompagnent quelles autres marchandises ?) transitent par le Rhône et doivent être embarqués plutôt à partir d'Arles que de Marseille ${ }^{216}$. De l'Italie parviennent d'assez abondantes sigillées tandis que les produits conditionnés en amphores sont à la fois peu nombreux et très divers : vin de Campanie (Dressel 1 et $2 / 4$ ) et de la côte adriatique (Lamboglia 2 et amphore de Forlimpopoli217). Ce sont les provinces orientales qui apparaissent avec le plus de netteté, la mer Égée (amphore rhodienne, LRA 2), l'Asie mineure (LRA 3), la Cilicie (LRA 1) et la Palestine (LRA 4). Selon un schéma désormais classique, le développement des importations orientales est un phénomène tardif.

On attendait surtout de la céramique qu'elle nous renseigne sur la nature des productions vivrières du littoral tunisien durant l'antiquité. En effet, pour quels produits les amphores africaines, dont plusieurs ateliers ont été repérés le long des côtes, étaient-elles prévues ? Le contenu des amphores africaines est une question qui a déjà fait couler beaucoup d'encre. Sans revenir en détail sur ce dossier ${ }^{218}$, nous retiendrons les points suivants :

a) Depuis leur mise en évidence en 1969, on soupçonne que les divers types d'amphores africaines ont pu avoir des contenus différents. Mais c'est l'huile qui a été le plus souvent proposée en raison des nombreuses données historiques sur l'arboriculture africaine.

b) Peu à peu, le doute s'est installé et il s'avère aujourd'hui qu'un grand nombre d'amphores africaines n'ont jamais transporté d'huile. L'un des critères retenus est la présence d'un enduit intérieur de poix. Cet enduit est considéré comme non seulement inutile mais même nuisible à un contenu oléagineux. Or après enquête dans la bibliographie et sur des amphores recueillies en mer (un milieu humide est nécessaire à la conservation de la poix), on constate que la majorité des amphores Africaines II, Keay XXV, Keay XXXV B et Keay LXI/LXII sont poissées. En

216. La sigillée luisante est rare à Marseille.

217. Associée à l'amphore gauloise dans la nécropole de Pupput (cf. note 189).

218. Se reporter à BEN LAZREG N. et al., Salsamenta, 1995. 


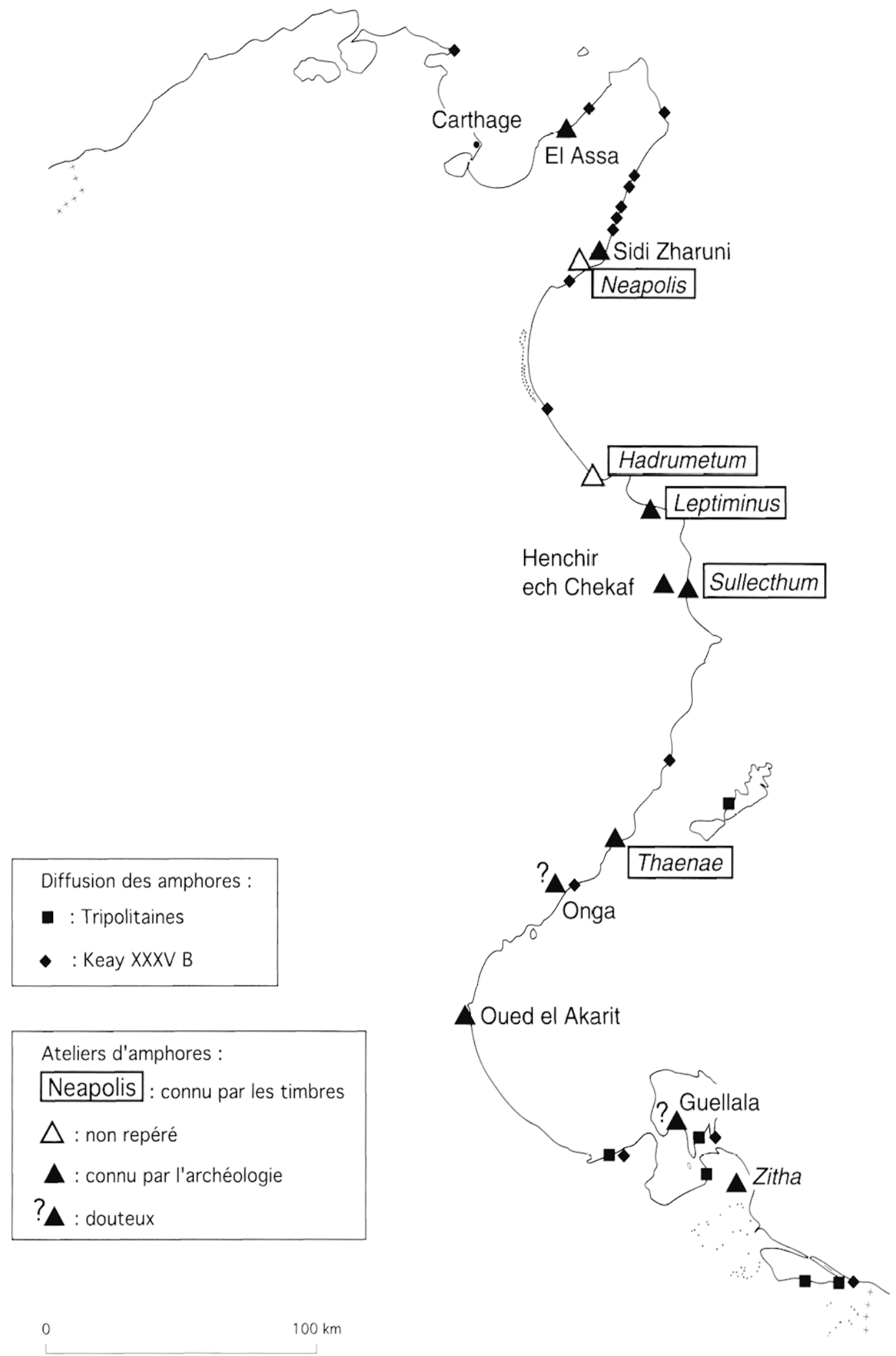

Fig. 22 : Production et diffusion de quelques types d'amphores africaines sur le littoral tunisien. (M. Bonifay) 
conséquence, seul le type Africaine I, systématiquement non poissé, peut être considéré comme un conteneur à huile.

c) L'huile une fois éliminée, il reste à choisir entre les deux autres principales denrées susceptibles d'avoir été transportées dans ces amphores : le vin ou les salaisons de poisson. Le vin pourrait être un bon candidat, d'autant qu'on a cherché à imiter de célèbres amphores vinaires à la fois au sud (Guellala : imitations de Dressel 2/4), au centre (Leptiminus : imitations de Dressel 1 (?))219 et au nord du littoral (El Assa et Nabeul : imitations d'amphores à fond plat Gauloises ou Dressel 30). Par ailleurs, une analyse par chromatographie gazeuse réalisée sur les résidus de résine d'une amphore Keay XXV.1 a donné un spectre infrarouge tout à fait comparable à celui obtenu sur une amphore Lamboglia 2 de l'épave de la Madrague de Giens dont on est sûr qu'elle contenait du vin. En ce qui concerne les salaisons de poisson, les seules informations disponibles proviennent des résidus de contenu : des écailles et des arêtes de poissons ont été retrouvées dans les amphores Africaines II de trois épaves. Enfin, certaines amphores africaines poissées (Africaine II et Keay XXV.2) ont très bien pu transporter des produits de l'oléiculture, notamment des olives conservées dans du defrutum.

d) Les recherches menées sur le littoral apportent de nouvelles informations sur trois types d'amphores :

- Le type Keay XXXV B. Cette amphore du ve s. a été produite, au moins en partie, sur l'atelier de Sidi Zahruni, à Beni Khiar, à $6 \mathrm{~km}$ au nord de Nabeul (fig. 13, $\mathbf{n}^{\circ}$ 171) et largement exportée (voir infra, la contribution de $\mathrm{Cl}$. Capelli). Tous les exemplaires retrouvés en milieu humide, notamment sur le rivage septentrional de la Méditerranée, sont poissés 220 . Une amphore d'Aguilas (Espagne), probablement du même type (il manque le bord), contenait des restes de maquereaux et de thons ${ }^{221}$. Enfin, cette amphore est particulièrement fréquente sur les sites qui bordent la lagune de Korba et qu'il y a tout lieu d'identifier comme des installations de salaison de poissons.

- Le type Africaine II A " con gradino ". Cette amphore est produite en plusieurs points du littoral

219. Opart A., North African Dressel 1, 1998.

220. BEN LAzREG N. et al., Salsamenta, 1995, fig. 10 ; of. également Saltamaria Cl., Dramont E, 1995.

221. PARFIA F., Marques, 1972. p. 107-108.
(Thaenae, Sullecthum, Leptiminus, Hadrumetum). Les exemplaires retrouvés en mer sont généralement poissés ${ }^{222}$. Les deux exemplaires graphiquement complets (fig. $20, \mathbf{n}^{\circ} \mathbf{2 8 7}$ ) dans le comblement d'un des bassins de l'usine de salaison de Nabeul au second quart du III $^{\mathrm{e}} \mathrm{s}$. ne sont peut-être pas de fabrication locale, mais le type est attesté parmi les productions de l'atelier de Sidi Aoun, sur le territoire de la cité223.

- Enfin, un dernier type d'amphore africaine était passé inaperçu jusqu'aux travaux récents conduits sur les ateliers d'amphores de Lamta-Leptiminus ${ }^{224}$. A. Opait a pu démontrer ${ }^{225}$ que ces ateliers, ainsi que d'autres proches de Salakta ${ }^{226}$, ont produit, à la fin du $\mathrm{I}^{\mathrm{er}} \mathrm{s}$. et au $\mathrm{II}^{\mathrm{e}} \mathrm{s}$., une imitation d'amphore "à garum " hispanique à pointe creuse (type Dressel 14) ${ }^{227}$. L'hypothèse émise par A. Opait selon lequel ces amphores africaines étaient destinées au transport des salsamenta se trouve parfaitement confirmée par la découverte, à El Jem-Thysdrus, dans la maison d'Africa ${ }^{228}$, d'une pointe appartenant à ce type d'amphore (fig. $20, \mathbf{n}^{\circ} \mathbf{2 8 9}$ ) qui contenait encore des restes de poissons ${ }^{229}$. Une amphore entière (fig. 20, $\mathbf{n}^{\circ} \mathbf{2 8 8}$ ), provenant d'une découverte fortuite effectuée à Salakta ${ }^{230}$, pourrait appartenir à une variante évoluée du type Leptiminus $\mathrm{I}^{231}$.

Il y a donc un faisceau d'arguments convergents pour attribuer ces trois types d'amphores au transport des salsamenta africains.

222. Blis Lazkeg N. et al., Salsamenta, 1995, fig. 8 ; cf. notamment Molchot D., Epave romaine " A ", 1970.

223. Ghalia T., Bonifay M., Capeld Cl., Sidi Zahruni, à paraître, fig. $8, n^{\circ} 37$.

224. STONE D., STIRLING; L. et BEN LaZRF.g N., Suburban land-use, 1998.

225. OPAIT A., Early Roman $\Lambda$ mphorae, 2000 : type Leptiminus I, pl. 1.5 .

226. Peacock D.P.S., Bex Lazkeg N. et Bfjaolit F., Roman Amphora Production, 1989, p. 188, fig. $11, \mathrm{n}^{\mathrm{N}} 1$ et 4.

227. Il pourrait tout aussi bien s'agir d'une variante locale damphore du type Carthage EA IV.

228. Je remercie M. Hédi Slim d'avoir bien voulu me confier, ainsi quà mon collègue Jean Piton, l'étude des céramiques de la maison d'Africa.

229. Il s'agit malheureusement d'écailles, à l'exclusion de tout ossement, ce qui rend difficile une détermination précise des espèces (expertise Myriam Sternberg, CNRS-CCJ).

230. Conservée au Musée de Salakta.

231. À rapprocher de OpAIT A., Early Roman Amphorae, 2000 , variante IC : pl. $5, n^{\circ} 22$ ? 


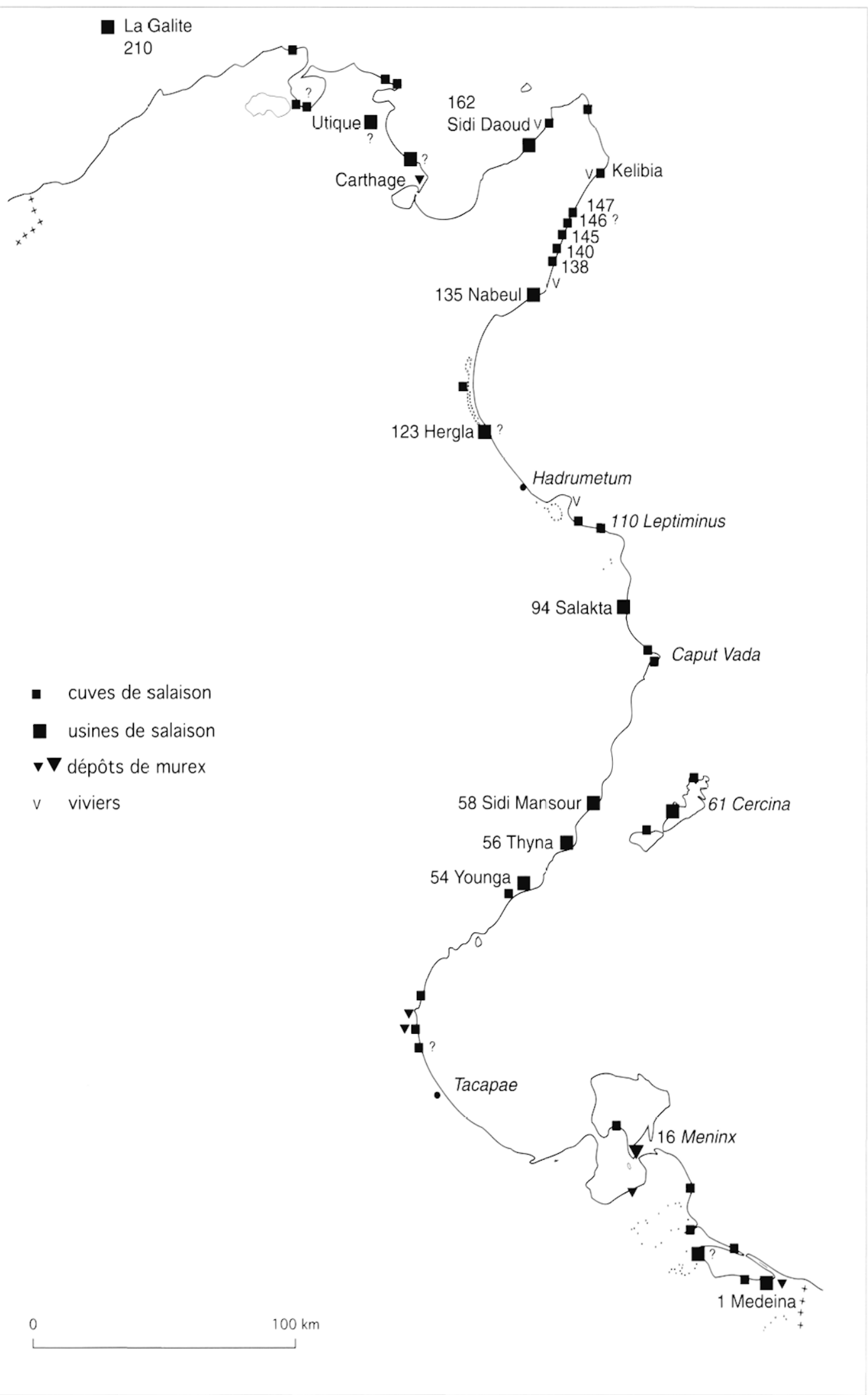

Fig. 23 : Traces d'usines de salaison repérées sur le littoral tunisien. (P. Trousset/J. Lenne) 
Céramiques de l'atelier de Portout (Chanaz, Savoie, F)

Exemplaires ACD 98 à 100, de Tunisie

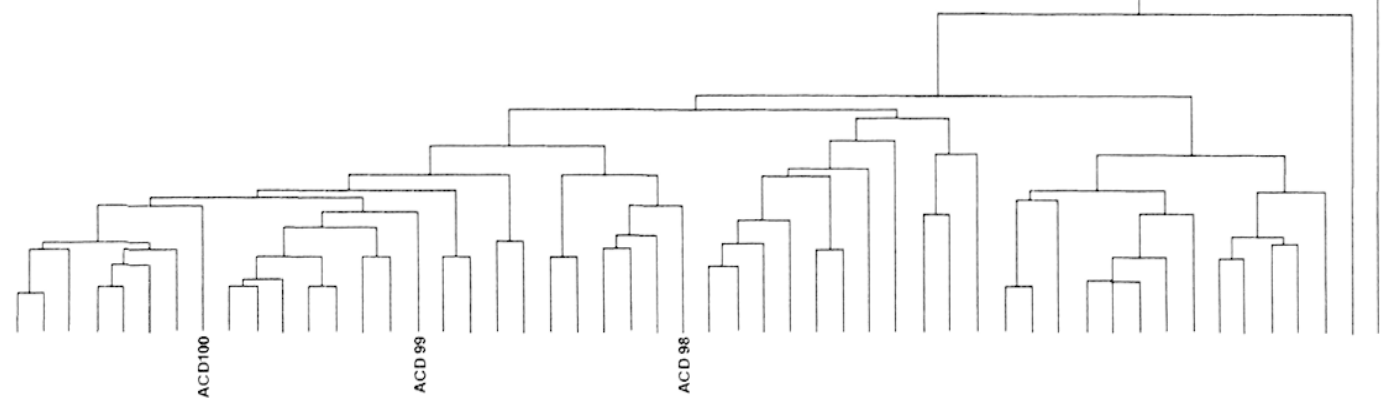

Fig. 24 : Dendrogramme de classification des sigillées luisantes recueillies sur le littoral tunisien. (M. Picon)

La question du contenu des amphores africaines est complexe; seul un programme ambitieux d'analyses pourrait prétendre résoudre le problème. Toutefois les recherches conjointes conduites sur le littoral tunisien et sur les amphores africaines exportées sur les côtes de Provence permettent de réévaluer le poids des diverses productions vivrières africaines $^{232}$. D'une part, l'huile occupe probablement une place moins importante que celle qu'on a bien voulu lui accorder par le passé. D'autre part, il n'est pas impossible que l'Afrique ait également exporté des quantités non négligeables de vin. Mais surtout, comptc tenu de la densité des installations halieutiques repérables par la présence de pesons de filets en céramiques (fig. 20, $\mathbf{n}^{\mathbf{o s}} \mathbf{2 8 3}$-285) ou d'hameçons en bronze (fig. $20, \mathbf{n}^{\circ} \mathbf{2 8 6}$ ) et surtout par l'existence de traces architecturales d'usines de salaison de poisson (fig. 23), on ne doit pas, au sein des exportations des provinces d'Afrique, sous-estimer le poids des salsamenta.

(M. B.)

\section{II - EXEMPLAIRES DE CÉRAMIQUE SIGILLÉE LUISANTE (TERRA SIGILLATA LUCENTE) EN TUNISIE \\ (par Maurice PICON)}

Trois exemplaires de céramique sigillée luisante recueillis au cours de prospections en Tunisie ont été examinés en laboratoire afin d'en vérifier l'origine. On

232. Tout au moins celles suceptibles d’être conditionnées en amphores. pouvait en effet supposer qu'il s'agissait d'exemplaires importés des ateliers de Savoie, et notamment de celui de Portout où cette production romaine tardive a fait l'objet de recherches chrono-typologiques et d'études en laboratoire 233 .

Ces exemplaires, identifiés ici par leur numéro d'analyse : ACD 98 à 100, sont les suivants :

- ACD 98 - Lamboglia 45, cf. fig. 4, n²3 (Inv. 037.10 ; provenance : Henchir Chougaf),

- ACD 99 - Lamboglia 1/3 ?, non illustré (Inv. 041.01 ; provenance : Sebkret el Guettiate),

- ACD 100 - Lamboglia 1/3, fig. 4, $\mathrm{n}^{\circ} 22$ (Inv. 055.01 ; provenance : Ilenchir Leich).

Les analyses ont été faites par fluorescence X et ont concerné 20 constituants chimiques. Mais seuls huit d'entre eux : $\mathrm{K}, \mathrm{Mg}, \mathrm{Ca}, \mathrm{Mn}, \mathrm{Al}$, Fe, $\mathrm{Si}$, et $\mathrm{Ti}$, ont été utilisés pour la classification destinée à comparer les compositions des exemplaires tunisiens aux références de l'atelier de Portout. Les analyses de ces dernières ne portaient en effet que sur ces huit constituants.

La classification a été effectuée par analyse de grappes (en affinité moyenne non pondérée, sur variables centrées réduites). Le dendrogramme correspondant (fig. 23) montre que les trois exemplaires tunisiens s'intègrent visiblement sans problème majeur parmi les références de l'atelier de Portout.

On notera toutefois que ces exemplaires ont tendance à se retrouver en position marginale dans les sous-groupes de l'atelier de Portout. Faible margina-

233. PICON M. et DFsBAT A., Sigillée claire B et luisante, 1986 : Perion J. et Chr., Portout, 1990. 
lité que confime le calcul de leur distance de Mahalanobis par rapport au groupe de référence de Portout. $\mathrm{Ce}$ calcul confirmant aussi leur très grande ressemblance avec ces références.

Les mêmes observations, en apparence contradictoires, ont déjà été faites sur les sigillées luisantes provenant de plusieurs sites d'habitat, en Gaule ${ }^{234}$. Elles doivent être interprétées comme la conséquence prévisible de l'existence d'ateliers ayant produit ce type de céramique, autres que celui de Portout, mais situés vraisemblablement dans la même région et dans le même contexte géologique. On connaît au moins deux d'entre eux, celui de Conjux qui se trouve à 2 ou $3 \mathrm{~km}$ de celui de Portout, sur les bords du lac du Bourget, et qui est actuellement immergé, et celui de Lucey sur la rive gauche du Rhône, à $8 \mathrm{~km}$ environ en aval de Portout. Sans doute peut-on imaginer qu'il en existe d'autres qui n'auraient pas été découverts, mais dont on retrouverait les productions sur les sites d'habitat. Il serait normal que leurs compositions présentent alors de fortes ressemblances avec celles de Portout, et qu'elles s'en distinguent aussi par quelques nuances de composition. En tout cas l'attribution aux ateliers de Savoie des trois exemplaires tunisiens ne semble faire aucun doute.

(M. P.)

\section{III - RICERCHE PETROGRAFICHE PRELIMINARI SULLE CERAMICHE " EOLICHE " (par Claudio CAPELLII235)}

\section{Introduzione}

Come è stato riconosciuto da tempo ${ }^{236}$, uno dei caratteri tipici di gran parte delle ceramiche prodotte

\footnotetext{
234. Desbat A., Pernon J. et Picon M., Terra sigillata lucente, 1990 ; Disbat A. et PiCON M., Sur l'origine des céramiques, 1994-95.

235. Il lavoro è stato realizzato nell'ambito del " Projet Mobilité du réseau des MSH : Caractérisation archéométrique des céramiques de l'Afrique antique : Recherche des lieux de production et reconstruction des circuits commerciaux ", svolto in collaborazione con la MOM di Lione (laboratoire "Archéométrie et Archéologie ") e la MMSH di Aix-en-Provence (Centre Camille Jullian).

236. ManNoNi T., Analisi mineralogiche II, 1972 ; II ., Analisi mineralogiche VI, 1979) P PEACOCK D.P.S., Petrology and origins, 1984.
}

presso le coste meridionali del Mediterraneo è rappresentato dalla particolare composizione dello scheletro degli impasti, il quale, al contrario di quanto si osserva in molti altri casi, risulta piuttosto povero dal punto di vista petrografico. Esso è infatti costituito essenzialmente da granuli di quarzo monocristallino, talora associati a frammenti di rocce calcaree e a microfossili a guscio carbonatico, mentre altri componenti che $s i$ trovano comunemente nelle altre produzioni mediterranee, come le miche, i feldspati e i clasti di rocce metamorfiche o magmatiche, sono in questo caso assenti $\mathrm{o}$ in quantità accessorie.

Un'altra qualità specifica di questo gruppo di impasti, ancor più determinante, è la morfologia di almeno una parte degli individui di quarzo di dimensioni superiori a $0.1-0,2 \mathrm{~mm}$, che presentano elevati gradi di arrotondamento e sfericità e le superfici tipicamente "smerigliate ". Tali caratteri, peculiari dei sedimenti eolici, derivano dalle numerose vicendevoli collisioni che i granuli subiscono nelle aree desertiche durante il trasporto operato dal vento, il quale, inoltre, esercita una forte selezione mineralogica e granulometrica dei sedimenti. La presenza della superficie opaca permette di distinguere il quarzo eolico da quello originato dall'elaborazione marina ; quest'ultimo, infatti, è privo di micropicchiettature ed appare quindi lucido e più o meno trasparente.

Nell'ambito del Mediterraneo, le aree di produzione dei manufatti realizzati con gli impasti sopra descritti, per i quali è stato definito il Gruppo mineropetrografico "Eolico " (E) ${ }^{237}$, appaiono localizzate nell'ambito delle aree costiere presso i deserti sabbiosi del Nord Africa, della Siria e della Palestina. Non risultano invece essere mai stati utilizzati per una significativa produzione ceramica i sedimenti eolici costieri, di limitata estensione, presenti anche in altre aree mediterranee ${ }^{238}$. E' quindi per ora sufficiente riconoscere in una ceramica di origine ignota la presenza di anche pochi individui di quarzo aventi i caratteri sopra descritti per suggerire una probabile origine nordafricana o mediorientale di quest'ultima. Come rimarcato dal Mannoni, tuttavia, è importante essere certi della determinazione dei caratteri " eolici ", per

237. Caplilu Cl. et ManNoni T., Proposte per una classificazione, 1997.

238. MaNoNi T., Analisi mineralogiche VI, 1979. 
non incorrere in errate ed infelici interpretazioni di provenienza239.

Se da un lato il riconoscimento delle ceramiche del Gruppo E è relativamente agevole nella maggior parte dei casi, d'altro canto la povertà di componenti dell'impasto (non soltanto dal punto di vista mineropetrografico, ma anche chimico) non fornisce informazioni utili per una più precisa localizzazione della loro provenienza nell'ambito sud-mediterraneo. Questo compito è reso ancor più difficoltoso da numerosi altri fattori, quali la limitata variabilità litologica del territorio (al contrario di quanto avviene in molti altri settori), l'insufficiente conoscenza dei luoghi e la loro scarsa accessibilità, la mancanza o la poca disponibilità di una cartografia geologica particolareggiata e, infine, la difficile reperibilità di campioni di terre, di scarti di fornace o di ceramiche di provenienza certa.

\section{Elementi per una classificazione degli impasti eolici in microscopia ottica}

Per i motivi sopra discussi, le distinzioni all'interno delle ceramiche "eoliche "e le indagini sulla loro provenienza devono essere effettuate con procedimenti diversi e più complessi rispetto a quanto avviene negli studi su altri tipi di impasti meglio caratterizzati, che si basano sull'analisi delle associazioni petrografiche presenti nello scheletro e sui confronti tra queste e la cartografia geologica.

In attesa di un esame approfondito e sistematico riguardante il Gruppo E, l'osservazione delle diverse centinaia di campioni di impasti eolici di epoca classica e medievale presenti in $\operatorname{archivio}^{240}$ ha permesso di individuare le principali variabili che potranno essere utili per eventuali classificazioni (indipendenti o meno da quelle archeologiche e dalla cronologia), distinzioni di gruppi di impasti e tentativi di localizzazione e caratterizzazione dei siti produttivi ${ }^{241}$ :

239. MANNoni T., Una precisazione, 1997.

240. La banca dati del DIP.TE.RIS. e dell'Istituto di Storia della Cultura Materiale (I.S.CU.M.) di Genova, realizzata dallo scrivente e, già a partire dagli anni ' 60 , da Tiziano Mannoni, è costituita attualmente da oltre seimila sezioni sottili di ceramiche mediterranee.

241. Si sottolinea comunque la difficoltà propria dei metodi in microscopia ottica - basati sulla soggettività e l'esperienza dell'analista, sull aspetto generale e sui confronti visivi - di fornire precise descrizioni e di elencare e quantificare singoli caratteri di un insieme. a) rapporto ferro/calcio diffusi nella matrice argillosa ;

b) percentuale di scheletro ;

c) grado di angolosità media dei componenti dello scheletro e frequenza di individui arrotondati - talora costituiti anche da minerali di diversa natura rispetto al quarzo, ma sempre in quantità subordinate rispetto a quella totale ;

d) grado di assortimento dello scheletro ;

e) dimensioni massime dello scheletro ;

f) dimensioni medie dei clasti delle diverse frazioni granulometriche ;

g) percentuali delle diverse frazioni granulometriche ;

h) eventuale presenza (e percentuale, tipo, dimensioni) di fossili242 ;

i) eventuale presenza (e frequenza, dimensioni e grado di arrotondamento) di calcari o calcareniti ${ }^{243}$;

j) eventuale presenza (e frequenza) di quarzo metamorfico (policristallino e/o monocristalli ad estinzione ondulata) ;

k) eventuale presenza di rocce metamorfiche quarzo-feldspatiche, vulcaniti, selci, arenarie, siltiti, argilliti, plagioclasio, K-feldspato, minerali pesanti (in genere anfibolo, tormalina, clinopirosseno, epidoto, zircone, titanite, rutilo, opachi).

Molti dei caratteri sopra elencati possono essere influenzati dagli interventi di alterazione delle argille naturali operati dai vasai, quali la loro decantazione e depurazione, le aggiunte di smagrante e il mescolamento di terre diverse ; forti cambiamenti possono inoltre essere causati dai diversi processi di cottura. Tali interventi, tuttavia, in quanto quasi mai riconoscibili con certezza, non possono essere considerati ai fini di una classificazione oggettiva, basata essenzialmente sul prodotto finito ${ }^{244}$. In altre parole, una stessa terra lavorata con metodi differenti darà probabilmente luogo a diversi sottogruppi di impasti, che comunque risulteranno più o meno legati tra loro a

242. E' importante riconoscere nell'impasto gli indizi di una presenza originaria di elementi carbonatici, anche quando i processi di cottura o di alterazione hanno condotto ad una loro dissociazione più o meno completa.

243. Vedi nota precedente.

244. Tale concetto risulta valido in generale e non solo per le ceramiche del gruppo $\mathrm{E}$. 
causa dell'origine comune di almeno una parte della materia prima.

Una classificazione parallela e complementare a quella relativa ai corpi ceramici può invece essere fatta per quanto riguarda i fattori sicuramente correlabili ai processi tecnologici, quali :

1) il grado di isorientazione dell'impasto e dei suoi componenti (vacuoli, minerali allungati o lamellari, ecc) ;

m) il colore dell'impasto e le sue eventuali variazioni nella sezione trasversale;

n) il grado di ossidazione del ferro diffuso ;

o) il grado di omogeneità dell'impasto ; se questo è basso, è importante riconoscere se è dovuto ad una cottura non omogenea oppure ad un'incompleto mescolamento di terre differenti ;

p) il grado di vetrificazione (stima approssimata) della matrice ;

q) la percentuale, le dimensioni, la forma, il grado di assortimento e di arrotondamento dei vacuoli, che possono derivare da gas intrappolati durante $\mathrm{i}$ processi di lavorazione e cottura dell'impasto, oppure da originari componenti dello scheletro più o meno dissociati ;

r) l'eventuale presenza di schiarimenti o arrossamenti superficiali ed il loro spessore ;

s) lo spessore, la composizione e la qualità dei rivestimenti eventualmente presenti.

Impasti con smagrante ben assortito, in prevalenza piuttosto angoloso e povero di quarzo eolico, potrebbero derivare da aree subdesertiche o da sedimenti di brevi corsi d'acqua o lacustri.

Un basso grado di assortimento, con la presenza di numerosi individui equidimensionali e arrotondati, non può invece far escludere l'aggiunta intenzionale di sabbia per smagrare un'argilla depurata.

Infine, la presenza più o meno abbondante di calcari o di microfossili (caso non raro nelle ceramiche del gruppo E), elementi molto teneri, suggerisce la vicinanza alle fabbriche di affioramenti di rocce sedimentarie più o meno incoerenti o alterate.

\section{Le ipotesi di provenienza}

Come già rimarcato in precedenza, le ricerche sull'origine delle ceramiche eoliche basate sui confronti tra la composizione dell'impasto e la cartografia geologica sono ostacolate sia dalla povertà composizionale degli impasti che da quella litologica e sedimentologica dei settori meridionali del Mediterraneo. Per quanto riguarda le componenti accessorie a quella eolica, si osserva come i calcari e le selci affiorano in molte aree, così come i sedimenti marnosi o argillosi fossiliferi sono molto diffusi presso le coste. Le rocce metamorfiche acide e le vulcaniti sono più scarse e localizzate (principalmente in Tunisia settentrionale e in Algeria nord-orientale le prime, in Tripolitania e nei bacini del Nilo e del Giordano le seconde), ma la loro presenza negli impasti eolici è in genere costituita da rari frammenti isolati, spesso arrotondati o molto piccoli, che potrebbero aver subito un lungo rimaneggiamento.

Nella banca-dati di Genova sono a disposizione, al momento attuale, quasi esclusivamente campioni relativi a ceramiche rinvenute nei siti di importazione. Anche nel caso si riuscisse a produrre un'efficace classificazione degli impasti eolici, la scarsità di elementi discriminanti sopra discussa non permetterebbe di correlare i singoli gruppi e sottogruppi riconosciuti alle aree produttive, e vi è la forte probabilità che impasti simili possano provenire da settori molto distanti.

L'unica soluzione possibile per ottenere significativi risultati nelle ricerche di provenienza sulle ceramiche nordafricane e mediorientali, da condurre sempre in stretta collaborazione con gli archeologi, risulta quindi quella di avere a disposizione una banca-dati di confronto costituita da scarti di fornace.

\section{Uno studio preliminare : le produzioni di alcune fornaci tunisine}

Per valutare le effettive potenzialità di una ricerca archeometrica, sulla base di quanto discusso finora, è stato effettuato uno studio preliminare su un numero limitato di campioni (dieci) di anfore (scarti di fornace) prelevati in sette siti produttivi della Tunisia (supra; Peacock et al. 1989), a cui è stato aggiunto un frammento di anfora di origine indeterminata proveniente dagli scavi di Marsiglia 245 .

245. BONIFAY M., Observations, 1986. 
In Tabl. II sono sintetizzati i principali caratteri petrografici osservati e i riferimenti archeologici relativi a ciascun campione.

Innanzi tutto, a conferma di quanto sopra discusso, tutte le ceramiche studiate possono essere attribuite al Gruppo Eolico. Emerge tuttavia un dato ancor più importante : un'attenta caratterizzazione petrografica permette, almeno in questo contesto estremamente limitato, di differenziare gli impasti di ogni sito dagli altri.

Il campione 5764 (Keay XXXVB, Sidi Zahruni) (fig. $25, n^{\circ} 1$ ) è ben distinto dalla presenza di una frazione fine $(<0,1 \mathrm{~mm})$ abbondante, costituita in prevalenza da quarzo angoloso (lo scheletro di maggiori dimensioni è invece piuttosto scarso e selezionato), e da una matrice ferrica non del tutto omogenea, in cui si notano inclusioni e strie di argilla ferrica (rosse) e, in percentuale minore, plaghe e strie di argilla carbonatica (chiare). Sono presenti calcari e fossili in quantità accessorie e rare miche fini.

Per alcuni versi simili tra loro appaiono gli impasti delle due anfore Keay LXII (5756 e 5761) (fig. 25, $\left.n^{\circ} 3\right)$ provenienti da Henchir ech Chekaf ${ }^{246}$, caratterizzati da uno scheletro mediamente abbondante e ben assortito, in cui sono presenti calcari e fossili relativamente numerosi. Non si esclude che le differenze possano essere solamente tecnologiche (lavorazione e grado di cottura, probabilmente maggiore in 5756) e che siano accentuate dalla forte presenza di carbonati di deposizione secondaria in $\mathbf{5 7 6 1 .}$

Il campione di Africana IID da Thyna (5760) (fig. $25, \mathrm{n}^{\circ} 4$ ) presenta una matrice ferrica opaca e uno scheletro ben assortito, piuttosto fine e costituito quasi esclusivamente da quarzo; non si esclude che alcuni vacuoli siano legati alla presenza originaria di microfossili e calcari dissociati dalla cottura.

I due impasti provenienti da Gallala (Jerba) (5758: produzione attuale, 5759 : Dressel 2/4 o Schone-Mau XXXV) (fig. 25, no 5 e 6), comunque distinti dagli altri, appaiono relativamente diversi tra loro. Il primo evidenzia una frazione di scheletro fine piuttosto abbondante ed una matrice non molto omogenea ; inoltre, è stato probabilmente cotto a temperature maggiori del secondo. Altri caratteri sono maggior-

246. PlacoCK D.P.S., BeN LaZRe, N N. e BejaOli F., Roman Amphora Production, 1989, 181. mente confrontabili e, soprattutto, i due campioni possono essere accomunati da una discreta percentuale di miche, inusuale nelle ceramiche eoliche.

Il campione 5762 (Keay XXV, El Maklouba) ${ }^{247}$ (fig. $25, n^{\circ} 7$ ) è caratterizzato da una matrice ferrica opaca, e da uno scheletro classato, con una frazione di quarzo di dimensioni molto fini fortemente separata da quella maggiore, che presenta un grado di arrotondamento piuttosto elevato ed è costituita, oltre che dal quarzo, da diversi frammenti di rocce calcaree più o meno dissociate.

I due impasti provenienti da Salakta (5755: Africana IIA, 5757 : Africana I) (fig. 25, no 8 e 9), pur presentando alcune differenze nella frequenza e nelle dimensioni dei componenti, sono accomunati da molti caratteri peculiari : tra questi, lo scheletro di dimensioni medio-fini, classato, costituito da quarzo in percentuali non elevate, da una grande quantità di fossili e calcari, poco dissociati dalla cottura, e da una componente di natura vulcanica, rappresentata in 5755 da rari frammenti di vetro rosato con inclusioni fini e, in 5757, da individui di clinopirosseno relativamente numerosi.

Il primo carattere che distingue l'impasto di Africana IIB (5763) di Lamta - Dahar Slima ${ }^{248}$ (fig. 25, $\left.\mathrm{n}^{\circ} 10\right)$ dagli altri è la composizione carbonaticoferrica, che è in relazione con il colore giallo-arancio del corpo ceramico. Tutti gli altri campioni analizzati in questa sede hanno invece una matrice in prevalenza ferrica, tendente quindi al rosso (a parte le superfici schiarite). Altri caratteri distintivi sono la presenza di rare inclusioni argillose rosse e di uno scheletro piuttosto abbondante, di dimensioni non elevate e mediamente assortito. Il quarzo, frequentemente arrotondato anche negli individui di piccole dimensioni, è prevalente su calcari e fossili.

$\grave{E}$ stato infine effettuato un primo test di confronto tra i campioni sopra descritti e l'impasto di un'anfora Keay XXXVB (5765) (fig. 25, $\mathrm{n}^{\circ} 2$ ) proveniente dagli scavi di Marsiglia, caratterizzato da una matrice ferrica, con alcune inclusioni argillose, ed uno scheletro mediamente assortito, ricco di quarzo, con una frazione fine particolarmente abbondante; calcari e fossili sono poco frequenti, e si notano alcune miche.

247. Ihid., 194.

248. Ibid. 197. 

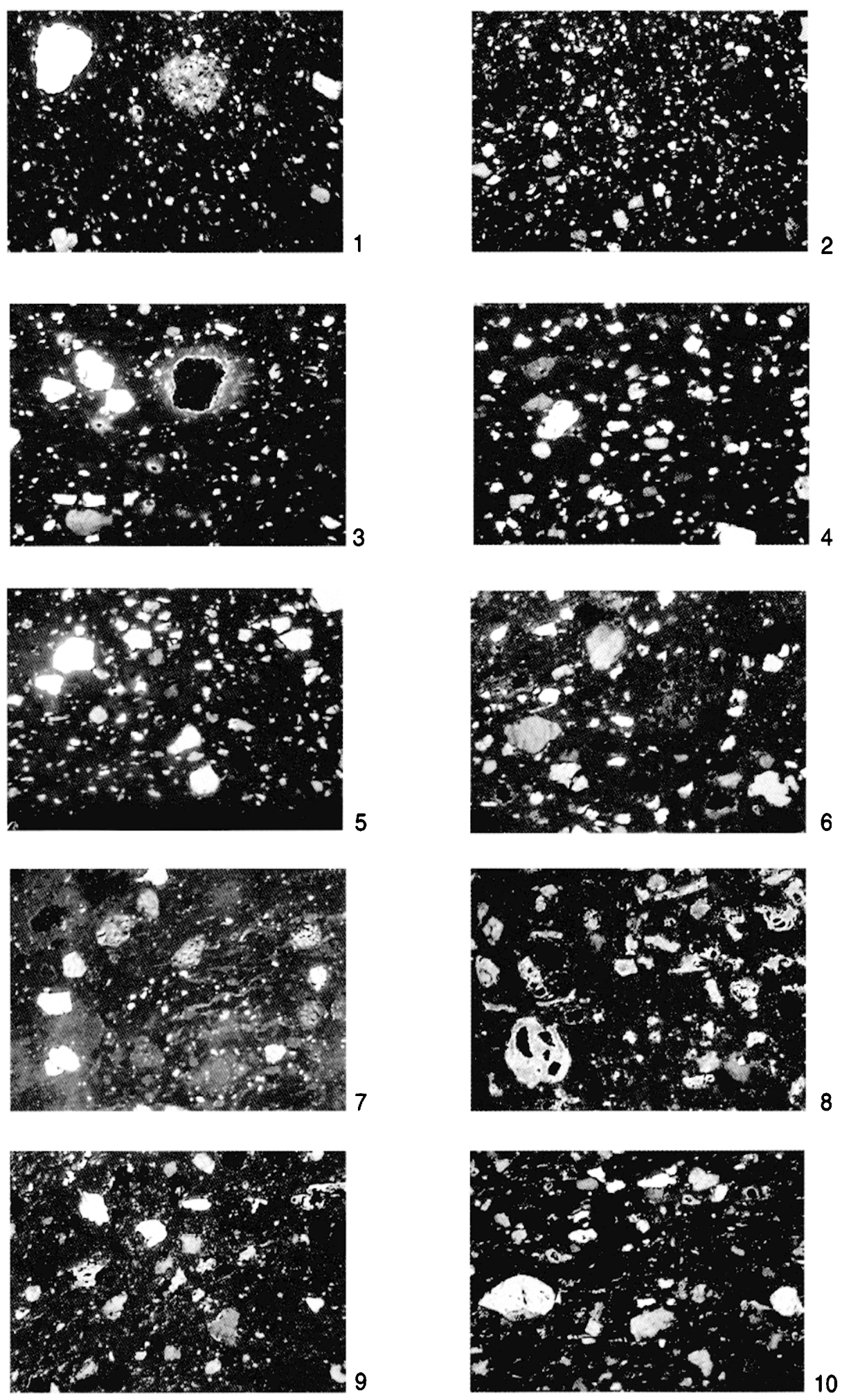

Fig. 25 : Fotografie al microscopio polarizzatore (nicol incrociati, area reale inquadrata : $1.3 \times 2 \mathrm{~mm}$ ) di alcuni degli impasti studiati. 1 : campione 5764 (Sidi Zahruni, tipo Keay XXXV B); 2 : campione 5765 (Marsiglia, tipo Keay XXXV B) ;

3 : campione 5756 (Henchir ech Chekaf, tipo Keay LXII) ; 4 : campione 5760 (Thaenae-Thyna, Africana II D) ; 5 : campione 5758 (Jerba-Gallala, attuale) ; 6 : campione 5759 (Jerba-Gallala, tipo Dressel 2/4 ou Schöne-Mau XXXV) ; 7 : campione 5762 (El Maklouba, tipo Keay XXV) ; 8 : campione 5755 (Salakta, Africana II A) ; 9 : campione 5757 (Salakta, Africana I) ; 10 : campione 5763 (Leptiminus-Dahar Slima, Africana II B). (Cl. Capelli) 
Pur esistendo alcune differenze, si notano molte analogie non solo composizionali, ma anche tecniche (ad esempio la presenza di un netto e sottile schiarimento superficiale) con il campione di anfora dello stesso tipo (5764) prodotta a Sidi Zahruni. In ogni caso, si può affermare che l'impasto risulta molto diverso da quelli relativi a tutte le altre aree produttive prese in esame.

\section{Considerazioni conclusive}

I primi risultati della nostra indagine sulle ceramiche nordafricane, basata su un'accurata analisi petrografica degli impasti dei siti produttivi, da integrare con quella tipologico-archeologica, possono essere considerati confortanti e indicano che la strada è percorribile, anche se con difficoltà. Viene quindi ad essere confermato quanto emerso dai lavori di D.P.S. Peacock ${ }^{249}$.

I prodotti di ognuno dei sette centri indagati, anche di diversa tipologia ceramica, appaiono infatti più o meno ben distinguibili dagli altri per diversi caratteri dello scheletro e della matrice (anche se, naturalmente, dovrà essere esteso anche il campionamento relativo a ciascun sito per verificare l'omogeneità della sua produzione o per definirne la variabilità). Tramite i confronti petrografici, viene inoltre indicata la possibile provenienza di un'anfora rinvenuta a Marsiglia da uno di tali centri (o, almeno, potrebbe essere esclusa la sua origine dai rimanenti).

I problemi cresceranno di molto, tuttavia, quando si estenderà il numero di siti e di tipi ceramici indagati ; data la povertà composizionale degli impasti eolici, è da supporre che le differenze, in molti casi, non saranno così evidenti. Solo poche fabbriche saranno probabilmente ben distinguibili e dovranno essere creati, negli altri casi, dei gruppi composizionali in cui rientreranno materiali di siti produttivi anche molto distanti tra loro. E' inoltre irrealizzabile, per ovvi motivi, una banca-dati completa relativa a tutte le fornaci che sono esistite.

I confronti permetteranno quindi solo in pochi casi fortunati di riconoscere la precisa provenienza di

249. PFACOCK D.P.S., Petrology and origins, 1984 ; PFACOCK D.P.S. e TOMBBER R., Roman amphoras kilns, 1991. una ceramica nordafricana o mediorientale trovata in siti di consumo. Anche la non-compatibilità di un impasto di origine sconosciuta con quelli di determinate aree produttive è comunque un dato importante, che permette, in ogni caso, di escludere determinate ipotesi archeologiche, favorendone altre.

Un parallelo filone di indagine dovrà riguardare le singole tipologie ceramiche, caratterizzando il maggior numero di campioni rappresentativi provenienti dai siti di consumo.

Da un lato dovranno essere ricercate, all'interno di una stesso tipo, le eventuali differenze non solo composizionali, ma anche tecniche, probabile indizio che una stessa forma veniva realizzata in diverse fornaci o aree produttive (si miglioreranno quindi le classificazioni basate solo sui dati morfologici), e dovrà essere valutata la distribuzione dei manufatti relativi ad ogni gruppo di impasti. Saranno quindi effettuati i confronti con i campioni di provenienza certa eventualmente disponibili.

Dall'altro lato, dovrà essere ricercata la presenza di analogie di impasto e tecnica di realizzazione in tipi o classi differenti, nel tentativo di caratterizzare l'insieme della produzione delle singole fornaci o aree produttive, anche se non ancora localizzate.

(Cl. C.)

\section{IV - CÉRAMIQUES D'ÉPOQUE ISLAMIQUE (par Lucy Vallauri)}

Cet échantillonnage de céramiques musulmanes a été effectué à partir de 7 sites côtiers dispersés du sud au nord, dans le golfe de Gabès (sites n'* 32, 43, 77, 79), près de Madhia (site $n^{\circ} 90$ ), sur la côte ouest du Cap Bon (site $n^{\circ} 165$ ), dans le Cap Blanc (site $n^{\circ} 197$ ) et jusqu'à l'île de Tabarka (site $n^{\circ} 209$ ). Il réunit une vingtaine de tessons qui, soit par la forme soit par la catégorie de revêtement, peuvent être rattachés à l'époque médiévale. L'inventaire a été dressé par site (cf. catalogue) mais les céramiques sont illustrées et classées par catégories sur une même planche. On retrouve ainsi sur la plupart des sites trois types de céramiques représentées ici en proportion égale.

La première catégorie qui domine généralement sur tous les sites de l'Ifriqya et du Maghreb est sans revêtement (fig. 26, nos 1-6). Les argiles sont proches de celles utilisées pour les vaisselles communes et les 
amphores, depuis l'antiquité jusqu'à la période contemporaine. Les pâtes de couleur beige, orangé ou plus rouge, sont souvent plus claires en surface. Ce phénomène est obtenu à la cuisson par l'adjonction d'eau salée. Souvent granuleuses ou feuilletées, elles contiennent de nombreuses particules de chaux et parfois des grains de quartz observés dans les classifications pétrographiques des céramiques antiques du nord et du centre de la Tunisie ${ }^{250}$.

Cette catégorie regroupe des vases de stockage pour l'eau, tels un col cylindrique à lèvre en bourrelet et un fond globulaire à ombilic rentrant (fig. 26, nos 12) qui peuvent appartenir à des amphorettes ou petites jarres à 2 anses. La seconde forme à col large, panse à carène et petit fond étroit (fig. 26, nos 3-5) comporte souvent une ou deux anses. Ce gros bol fait partie également du répertoire glaçuré ou peint. L'unique forme ouverte, tronconique et à petit marli (fig. $26, \mathbf{n}^{\circ} \mathbf{6}$ ), peut avoir été utilisée comme coupe ou bassin mais également comme couvercle comme l'atteste un noircissement sur le bord. Les céramiques communes sont encore difficiles à dater car les formes ont perduré sans grand changement et lorsqu'elles sont très fragmentées il est difficile de séparer les productions récentes de celles médiévales, tardo-antiques et byzantines. Trop souvent délaissées dans les publications au profit des vaisselles monochromes glaçurées ou de celles plus luxeuses peintes, elles ont rarement fait l'objet d'étude systématique. Des amphorettes et de gros bols à anse aux profils très similaires ont cependant été reconnus parmi le matériel fatimo-ziride découvert près de Kairouan à SabraMansuryá251, à Rougga ${ }^{252}$, à Sétif ${ }^{253}$ ou à Achir dans le Maghreb central254.

La seconde catégorie concerne les céramiques revêtues de glaçure monochrome vert turquoise et plus exceptionnellement vert sombre (fig. 26, nos 711). Les pâtes employées sont dans ce cas plus épurées et de couleur beige clair ou rosé ; néanmoins

250. PEACOCK D.P.S., Petrology and origins, 1984.

251. AJJABI H., La céramique de Sabra-Mansurya, 1992-93, p. 73-74 $n^{\prime \prime 1} 1-2 ; 79$ n"13-16; 70, $71 n^{\prime \prime \prime ~ 7-9 . ~}$

252. GuÉRY R., Stratigraphie du forum, 1984 ; GLÉRY R., La survivance de la vie sédentaire, 1985 ; VAlLAL RI L., La céramique d'époque islamique, à paraître.

253. Ama.mRa A.A., La céramique islamique, 1991, fig. $64 \mathrm{n}^{205}$ 6-7.

254. Mokra.i M.-A.. A propos des céramiques, 1997, fig. 26-39 et 65. elles contiennent souvent des petites particules de chaux. Les formes identifiées appartiennent au luminaire et à la vaisselle de service. Deux fragments de long canal aux parois facettées et profils en $U$ bien caractéristique évoquent le réservoir des lampes à huile (fig. $26, \mathbf{n}^{\circ} 7$ ). La fragmentation ne permet pas de les rattacher à un type précis de lampe dont la forme du corps, la préhension ou le goulot peuvent être très variés. Néanmoins, ces lampes à longs becs caractérisent les niveaux $\mathrm{X}^{\mathrm{e}}$-XII $\mathrm{e}$. de nombreux sites de l'Ifriqya, comme par exemple ceux de Raqqada, Sabra-Mansurya 255 , de Carthage ${ }^{256}$, Dougga 257 , Rougga ${ }^{258}$, Sétif 259 ou encore du Maghreb central, à la Qualaa des Beni Hammad et Achir ${ }^{260}$. À l'exception d'un fragment de panse cannelée appartenant à un petit vase à liquide, les fragments de pied annulaire haut ainsi que le petit fond bas (fig. 26, nos 8-9) appartiennent à plusieurs coupes. Celles-ci peuvent avoir une paroi marquée par une carène avec un rebord effilé (fig. $26, \mathbf{n}^{\circ} \mathbf{1 0}$ ) et une autre un bord en petit marli (fig. $26, \mathbf{n}^{\circ} \mathbf{1 1}$ ). Ces bassins largement ouverts sont bien connus dans le répertoire à décor polychrome peint sur des fonds jaunes ou opacifiés à l'étain à Sabra-Mansurya ${ }^{261}$, Carthage ${ }^{262}$, Rougga, ou encore à Ajdabiyah en Lybie ${ }^{263}$. Les mêmes formes monochromes à paroi redressée ou avec un petit marli se retrouvent aussi sur les façades des églises de Pise où elles ont été incrustées au XI $\mathrm{s}$. et début du $\mathrm{XII}^{\mathrm{e}} \mathrm{s}$. sur les édifices en construction ${ }^{264}$. La diffusion de cette vaisselle monochrome vert turquoise a été récemment attestée à Marseille dans des niveaux de la seconde moitié du XII ${ }^{\mathrm{e}}{ }^{265}$. Quant aux coupes à marli

255. DaOUlatli A., La production vert et brun, $1995, \mathrm{n}^{20 .} 30-31$; AJJABI H., La céramique de Sabra-Mansurya, 1992-93, p. 39-42, n ${ }^{\circlearrowleft \%} 1-$ 11.

256. Vitel.u G., Islamic Cartbage, 1981, fig. 59.

257. LouHichI A., La céramique islamique de Dougga, 1998, p. 122, 123, fig. 7, 8, 24.

258. Vallacro L., La céramique d'époque islamique, à paraître.

259. Amamra A.A., La céramique islamique, 1991, p. 227, fig. 68 , nos 2-6.

260. Golvis L., Les céramiques émaillées, 1981, p. 210, fig. 7 ; MOKRANI M.-A., À propos des céramiques, 1997, p. 300, n"s 111-113. 261. DaOliatli A. et Rammah M., La céramique ifriqienne, 1994, forme 2.2 et forme non répertoriée cat. $\mathrm{n}^{\circ} 72$.

262. Vitril.. G., Islamic Carthage, 1981, forme 1.1

263. RuIl:Y J., Ajdabiyah, 1982.

264. BiRTI G. et TONGIORGi L., I bacini ceramici, 1981 ; BerTI G., Ceramiche islamiche. 1991.

265. Renseignement Fl. Parent (AFAN Méditerranée). 
à glaçure vert sombre sur pâte rouge, elles sont bien diffusées en Provence et Ligurie tout au long du XIII' siècle ${ }^{266}$.

La dernière catégorie regroupe les fragments peints en vert et brun sur un fond de glaçure plombifère ou stannifère blanc (fig. $26, \mathbf{n}^{\text {os }} \mathbf{1 2 - 1 3}$ ) ou coloré au vert de cuivre (fig. 26, nos 14-15). La présence de l'étain n'est pas toujours assurée et bien que la présence d'un engobe soit désormais peu probable au vu des analyses effectuées sur le matériel tunisien retrouvé en Italie ${ }^{267}$, il peut s'agir aussi dans certains cas de surface blanchie à la cuisson. Le petit vase globulaire (fig. 26, nos 12-13) à décor de taches vertes encadrées de bandes brunes peut évoquer des formes avec anse et goulot ou sans anse ni col, comme celles conservées à Raqqada, Sabra-Mansurya ou à Carthage, Uchi Maius ${ }^{268}$. Les fragments de panses de formes ouvertes sur lesquelles sont peints généralement de larges motifs de divers styles, ne sont pas identifiables (fig. 26, nos 14-15).

La datation tout comme la provenance de ces échantillons demeurent floues compte tenu de leur condition de découverte (cf. infra), de la quasi absence de fouille de fours ou d'ateliers médiévaux, et du manque de références stratigraphiques précises pour les périodes islamiques ${ }^{269}$. La multiplication des études céramologiques montre cependant tout l'intérêt porté à ces céramiques d'époque médiévale devenues aujourd'hui plus familières grâce aux expositions et aux publications faites à partir des grands sites princiers de l'Ifriqya et du Maghreb central. Ces études de matériel prestigieux sont maintenant complétées par de nouvelles données sur l'ensemble des vaisselles retrouvées, communes aussi bien que décorées. L'analyse de petits ensembles de céramiques médiévales retrouvées sur des sites antiques réoccupés complète cette approche tout comme celle

266. VAll.Al'RI L., Les céramiques importées, 1997 ; AMOURIC H., Richi: Fl. et Vallat RI L., Vingt mille pots, 1999.

267. BiRTl G. et MANNON T. Rivestimento, 1990 ; BertI G., Ceramiche islamiche, 1991.

268. DAOL LATl. A. et RaMmah M.. La céramique ifriqienne, 1994, $\mathrm{n}^{\circ} 76$; DAOIl.ATLI A., La production vert et brun, 1995, n⿳亠丷厂 15 et 37 ; VITFil.I G., Islamic cartbage. 1981, p. 104, fig. 43 ; GH:LICHI S. et MILANes: M.. Uchi Mains, 1997. fig. $40 \mathrm{n}^{\circ} 2$.

269. DaOSIATl.J A.. La céramique ifriqiyenne, 1980 ; ID., La production vert et brun, 1995: II). La céramique médiévale en Tunisie, 1995 ; Lothich A.. La céramique fatimide et ziride. 1997. des séries islamiques diffusées en Italie et dans le Midi de la France.

L'existence de plusieurs centres de production médiévaux en Tunisie est reconnue en particulier dans l'aire de Kairouan, par les analyses physicochimiques effectuées sur du matériel glaçuré et émaillé provenant de Kairouan, d'Al Abbassia, de Raqqada et de Sabra-Mansurya. D'autres centres sont pressentis autour de Tunis, mais la similitude de composition des céramiques trouvées à la Kasba de Tunis avec celles de la région de Kairouan montre toute l'ampleur du problème de même que la probabilité d'une diffusion des vaisselles kairouanaises jusqu'à Tegdaoust en Mauritanie ${ }^{270}$. Les découvertes de fours à Sabra-Mansurya et à Madhia ne sont pas encore exploitées et il est difficile de savoir quelles catégories de céramiques ces ateliers produisaient, s'ils étaient polyvalents et à quelle période ils fonctionnaient.

Les comparaisons typologiques esquissées permettent cependant de rattacher avec prudence ces quelques tessons à une large séquence fatimo-ziride comprise entre le $\mathrm{X}^{\mathrm{e}}$ et le $\mathrm{XII}^{\mathrm{e}} \mathrm{s}$. L'absence de céramique peinte sur fond jaune " dite jaune de Raqqada " attribuée à la période aglhabide au IXe $s$. tout comme l'absence de faïence d'époques almohade et hafside peinte en bleu de cobalt et brun de manganèse, qui caractérise le XIII et le XIVe s., plaideraient en faveur de cette fourchette chronologique.

Le lot ramassé sur l'île de Tabarka doit être séparé de ce groupe, car il est à l'évidence d'époque moderne. Il regroupe des céramiques glaçurées de Pise à décor de stecca, importées aux XVI ${ }^{\mathrm{e}}$ et XVII ${ }^{\mathrm{e}} \mathrm{s}$. Elles sont très comparables à celles déjà ramassées lors d'une prospection ancienne ${ }^{271}$. Il n'est pas étonnant de retrouver sur cette île occupée par des familles ligures, des produits italiques qui circulent au sein d'un commerce triangulaire sur toutes les côtes méditerranéennes ${ }^{272}$.

270. Lothichi A. et PICON M., Importation de matériel céramique, 1983 ; Lot.HCH A., Ln échantillonnage, 1992-93 ; $/ \mathrm{l}$., La céramique fatimide et ziride, 1997.

271. Lotricil A., La céramique de l'lle de Tabarka, 1992-93.

272. Ayotric H. et ABE.t. V., Un goût d'Italie, 1993. 

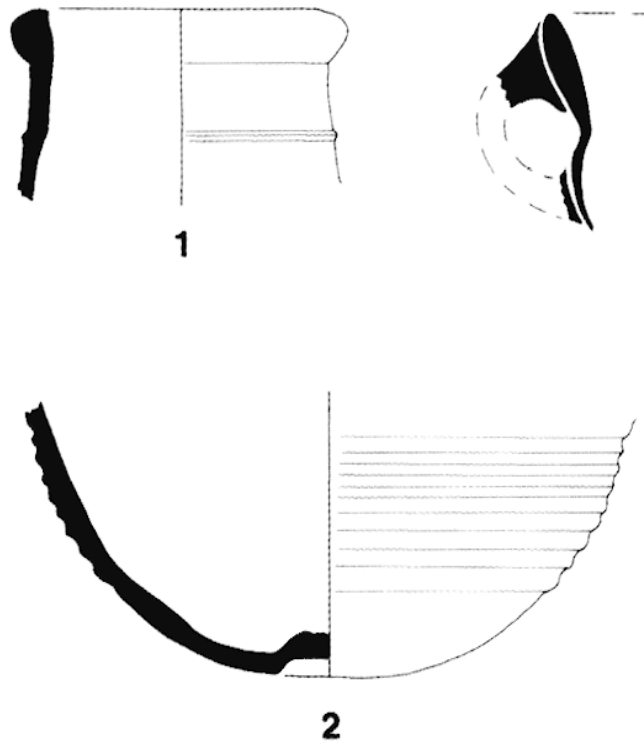

2

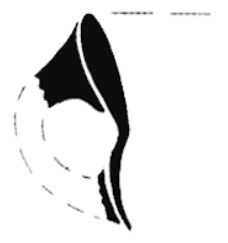

3

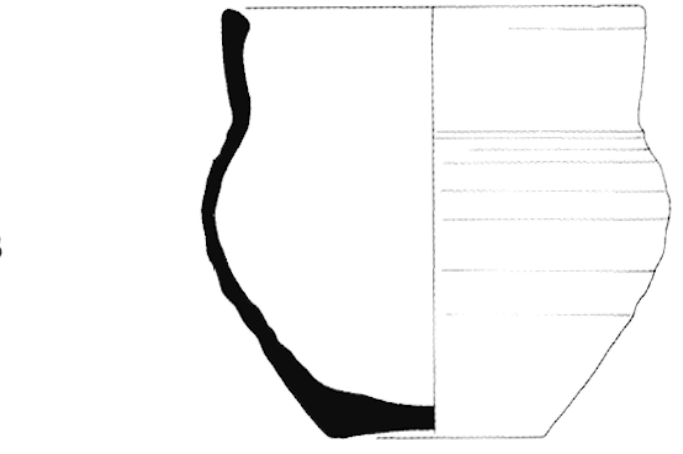

4

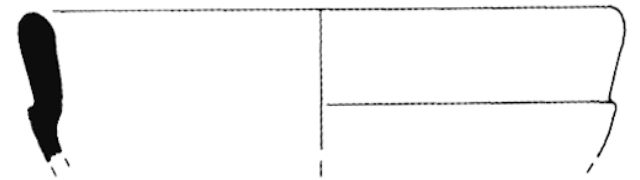

5

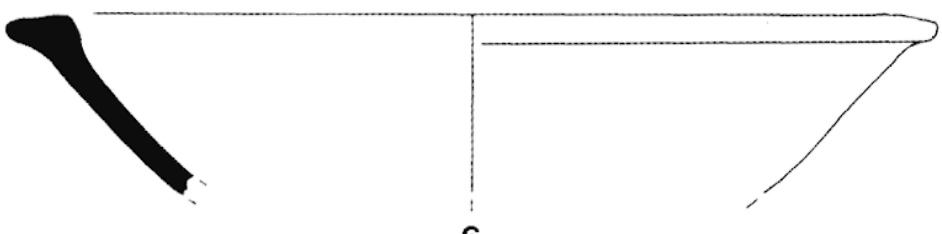

6

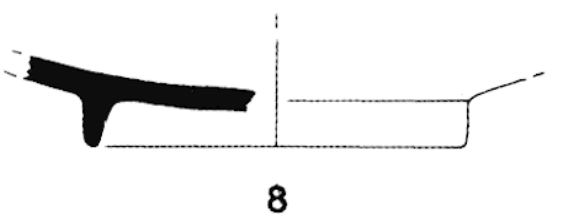

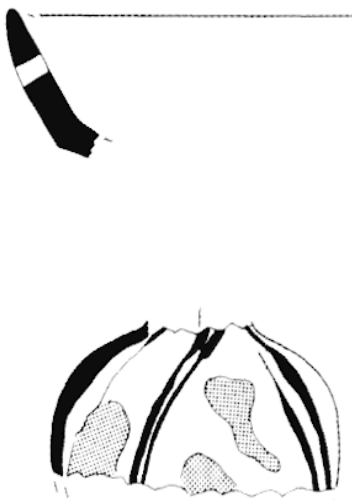

12

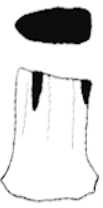

13

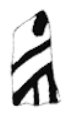

14

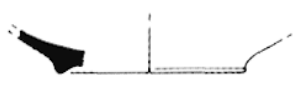

9

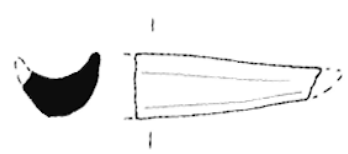

7

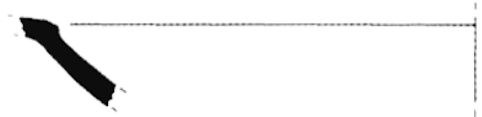

scm

11

Fig. 26 : Céramiques islamiques. (L. Vallauri) 


\section{Catalogue}

\section{Site $\mathbf{n}^{\circ} 32$ : Gourine est}

- bord de coupe à carène avec trou de réparation (fig. 26, n' 10)

pâte beige à surface brun rouge

glaçure vert turquoise écaillée à l'avers et au revers

- panse de petit vase cannelé (non dessiné)

pâte beige avec des nodules de chaux

coulures de glaçure vert turquoise à l'extérieur et blanchâtre à l'intérieur

- fragment de canal de lampe, profil en U (fig. 26, $\mathbf{n}^{\circ} 7$ )

pâte beige et rose en surface avec des vacuoles

glaçure vert turquoise

- fragment de canal de lampe (non dessiné)

pâte beige orangée criblée de paillettes de chaux

glaçure vert turquoise

\section{Site $n^{\circ} 43$ : La Skhirra}

- gros bol à fond plat étroit, col droit, lèvre épaissie, panse cannelée marquée par une carène, muni sans doute d'anse (fig. 26, $\mathbf{n}^{\circ} \mathbf{4}$ )

pâte beige sans revêtement

\section{Site $\mathbf{n}^{\circ} \mathbf{7 7}$ : Louza sud}

- fragment de coupe à marli (fig. 26, $\mathbf{n}^{\circ} \mathbf{1 1}$ )

pâte rouge brique criblée de particules jaunes de chaux glaçure épaisse au revers et à l'avers de couleur vert foncé

\section{Site $\mathbf{n}^{\circ} 79$ : Sidi Saad}

- fragment de panse de coupe à paroi épaisse de $1 \mathrm{~cm}$ (roulé ou retaillé) (fig. $26, \mathbf{n}^{\circ} \mathbf{1 5}$ )

pâte rouge orangée, avec des inclusions brunes

trait peint à l'intérieur au brun de manganèse sur fond de glaçure vert clair, et bande vert foncé à l'avers et au revers

\section{Site $n^{\circ} 90$ : El Alia Sebka Njila}

- plat tronconique à lèvre aplatie ou couvercle noirci sur le bord (fig. $26, \mathbf{n}^{\circ} \mathbf{6}$ )

pâte rouge orangée à surface claire, contenant de gros points de chaux, sans revêtement

- fragment de panse de coupe à carène (non dessiné) pâte beige à surface claire couverte par une glaçure vert clair à l'avers et au revers

- fond de coupelle à petit pied concave (fig. 26, $\mathbf{n}^{\circ}$ 9) pâte beige rosé à surface claire couverte par une glaçure vert clair à l'avers et au revers

- fragment de col de vase (non dessiné)

pâte rose orangée à surface claire

trait peint en brun à l'extérieur sur fond de glaçure vert turquoise qui couvre aussi l'intérieur

- fragment de panse de coupe à paroi épaisse (fig. 26, $\mathbf{n}^{\circ}$ 14)

pâte rose orangée à surface claire

décor peint à l'intérieur en brun sur fond de glaçure jaune et rehaussé de vert, glaçure jaune au revers

\section{Site $\mathbf{n}^{\circ} 165$ : Mraïssa}

- bord de gros bol à panse carénée (fig. $26, \mathbf{n}^{\circ} \mathbf{5}$ )

pâte beige rosé avec de petits grains bruns

- fond de coupe sur pied haut (fig. $26, \mathbf{n}^{\circ} \mathbf{8}$ )

pâte beige fine recouverte à l'avers et au revers par une glaçure monochrome vert turquoise

- petite anse rubanée de vase à liquide (fig. $26, \mathbf{n}^{\circ}$ 13)

traits bruns peints sur une glaçure stannifère?

- deux panses d'un petit vase globulaire (fig. $26, \mathbf{n}^{\circ} \mathbf{1 2}$ )

pâte rose violacée avec des vacuoles et des petits grains bruns

sur la glaçure externe opacifiée à l'étain ?, décor de traits bruns de manganèse et de taches vertes ; absence de revêtement à l'intérieur

\section{Site $\mathbf{n}^{\circ} 197$ : Rass Enghela}

- rebord de gros bol à lèvre allongée et carène avec départ d'une anse (fig. 26, $\mathbf{n}^{\circ} \mathbf{3}$ )

pâte?

- col de petite amphore à lèvre arrondie et ressaut sur le col pâte beige orangée, surface chamois sans revêtement (fig. 26, $\mathbf{n}^{\circ} \mathbf{1}$ )

- fond d'amphore à ombilic rentrant et panse cannelée (fig. 26, $\mathbf{n}^{\circ} \mathbf{2}$ )

pâte rouge assez fine, dure bien cuite avec des inclusions blanches, surface beige rugueuse sans revêtement

- fragment d'un fond de coupe sur pied haut (non dessiné) pâte?

décor peint vert et brun sur fond de glaçure blanche, glaçure verte et blanche à l'extérieur.

(L. V.) 


\section{BIBLIOGRAPHIE}

AA. VV. (Groupe de travail sur les Sigillées claires), 1986, Céramiques tardives à revêtement argileux des Alpes du nord et de la vallée du Rhône (de Martigny à Vienne), Figlina, 7 , p. $19-49$.

AJJABI H., 1992-93, La céramique de Sabra-Mansurya, Africa, XI-XII, p. 7-81 (en arabe).

AKerraz A., El-Khatib-BoujiBar N., Hesnard A., Kermorvant A., LENOIR E. et M., 1981-82, Fouilles de Dchar Jdid, 19771980, BAM, XIV, p. 169-246.

Amamra A.A., 1991, La céramique islamique, dans A. Mohamedi, A. Benmansour, A.A. Amamra et E. Fentress., Fouilles de Sétif 1977-1984, Alger (BAA, supplément 4), p. 206-228.

AmAR G. et LIOU B., 1984, Les estampilles sur amphores du golfe de Fos, Archaeonautica, 4, p. 145-211.

AMOURIC H. et ABEL V. (dir.), 1993, Un goût d'Italie : céramiques et céramistes italiens en Provence du Moyen Âge au $X X^{e}$ siècle, Catalogue de l'exposition, Aubagne.

Amouric H., Richez Fl. et Vallauri L., 1999, Vingt mille pots sous les mers. Le commerce de la céramique en Provence et Languedoc du $X^{\prime}$ au XIX'siècle, Catalogue de l'exposition (Musée d'Istres, 1999), Aix-en-Provence.

AQUILUÉ X., 1992, Sobre algunas cerámicas de producción africana con decoración espatulada, Revista d'Arqueologia de Ponent, 2, p. 177-198.

AQUilué X., 1993, Las cerámicas finas de los liveles tardoromanos, dans X. Dupré i Raventos et J.M. Carrete i Nadal (dir.), La Antiga Audiencia. Un accesso al foro provincial de Tarraco, Madrid (Excavaciones arqueologicas en España, 163), p. $117-150$.

ARThur P., 1982, Amphora Production in the Tripolitanian Gebel, Libyan Studies, 13, p. 61-72.

Ballet P., Mahmoud F., Vichy M. et PiCon M., 1991, Artisanat de la céramique dans l'Égypte romaine tardive et byzantine. Prospections d'ateliers de potiers de Minia à Assouan, Cabiers de la Céramique Égytienne, 2, p. 129-144.

BARADEZ J., 1961, Nouvelles fouilles à Tipasa, Libyca, IX, p. 7-195.

Barrald D., Bonifay M., Dridi F. et Pichonneau J.-F., 1998, L'industrie céramique de l'Antiquité tardive, dans $\mathrm{H}$. Ben Hassen et L. Maurin (dir.), Utbina (Oudbna), La redécouverte d'une ville antique de Tunisie, Bordeaux-Paris-Tunis, p. $146-167$.

BATS M., 1988, Vaisselle et alimentation à Olbia de Provence ( $v$. 350-v. 50 av. J.C.). Modèles culturels et catégories céramiques, Paris (RAN, Supplément 16).
Ben ABE[) A., Bunllay M. et Fixư M., avec la collaboration de Michel, D'ANNoville C. et Reynaud P., 1997, Note préliminaire sur la céramique de la basilique orientale de Sidi Jdidi (Tunisie) ( $\mathrm{V}^{\mathrm{C}}-\mathrm{VII}^{\mathrm{C}} \mathrm{s}$.), dans La céramique médiévale en Méditerranée, Actes du VI' Congrès de l'AICM2 (Aix-enProvence, 13-18 novembre 1995), Aix-en-Provence, p. 1325.

Berti G., 1991, Ceramiche islamiche del Mediterraneo occidentale usate come "bacini " in Toscana, in Sardegna e in Corsica (secoli XI-XIII), dans S. Scuto (dir.), L'età di Federico II nella Sicilia Centro Meridionale, Gela, p. 99-334.

BERTI G. et MANNONI T., 1990, Rivestimento vetrosi e argillosi su ceramiche medievali e risultati emersi da ricerche archeologiche e analisi chimiche e mineralogiche, dans T. Mannoni et A. Molinari (dir.), Scienze in Archeologia, Sienne-Florence, p. 89-124.

BerTI G. et TONGIORGI L., 1981, I bacini ceramici medievali delle chiese di Pisa, Rome.

BIEN St., 1998, Contextes de l'Antiquité tardive sur le chantier du Parc des Phocéens (îlot $24 \mathrm{~N}$ ), dans M. Bonifay, M.-B. Carre et Y. Rigoir, Fouilles à Marseille. Les mobiliers (Fer-VII s.), Paris (Études Massaliètes, 5), p. 275-283.

Blanc-Bijon V., CARre, M.-B., Hesnard A. et TChernia A., 1998, Recueil des timbres sur amphores romaines, II, Aix-enProvence.

Bonifay M., avec la collaboration de Pelletiek J.-P., 1983, Éléments d'évolution des céramiques de l'Antiquité tardive à Marseille d'après les fouilles de La Bourse (1980-81), RAN, XVI, p. 285-346.

BONIFAY M., 1986, Observations sur les amphores de Marseille d'après les fouilles de la Bourse (1980-1984), RAN, XIX, p. 269-305.

BONIFAY M., 1998, Sur quelques problèmes de datation des sigillées africaines à Marseille, dans Ceramica in Italia : $V$ VII secolo, Atti del Convegno in onore di Jobn W. Hayes (Roma, 11-12 maggio 1995), Florence, p. 71-82.

BONIFAY M., 2004, Observations préliminaires sur la céramique de la nécropole de Pupput, dans A. Ben Abed et M. Griesheimer (dir.), La nécropole romaine de Pupput, Rome, p. 21-57.

Bo.vifay M., 2004, Amphores de tradition punique du golfe d'Hammamet, dans A. Ben Abed et M. Griesheimer (dir.), La nécropole romaine de Pupput, Rome, p. 197-238.

Bonifay M. et PIFri D., 1995, Amphores du ve au vile s. à Marseille : nouvelles données sur la typlogie et le contenu, $J R A, 8$, p. 94-120. 
BOLBE J., 1965, La Terra Sigillata bispanique en Maurétanie Tingitane, 1 - Les marques de potiers, Tanger (Études et travaux d'archéologie marocaine, 1).

Bourgeols A., 1982, La céramique du Sanctuaire d'Hoter Miskar à Mactar, Karlbago, XX, p. 17-69.

Bourgeols A., 1986, La céramique sigillée de la Graufesenque en Afrique Proconsulaire, dans Mélanges offerts à $M$. Michel Labrousse (Pallas hors série), Toulouse, p. 323-334.

BotjRgrols A. et MAYET Fr., 1991, Belo VI. Les sigillées, Madrid.

BuCHI E., 1975, Lucerne del Museo di Aquileia. I, Lucerne romane con marchio di fabbrica, Aquilée.

Capelli C. et Maninoni T., 1996, Proposte per una scheda descrittiva delle sezioni sottili e per una classificazione minero-petrografica delle ceramiche, Archeologia Medievale, XXIII, p. 689-697.

Capelli C. et ManNoni T., 1997, Proposte per una classificazione degli impasti ceramici mediante gruppi mineropetrografici dello scheletro, correlabili alla geologia delle aree produttive, dans Atti del Convegno: Le Scienze della Terra e Archeometria, Naples, p. 123-125.

Carandini A., 1967-68, Sigillata Africana, dans A. Carandini (dir.), Ostia I. Le Terme del Nuotatore. Scavo del ambiente $I V$, Rome (Studi Miscellanei, 13), p 25-61.

Carandini A. (dir.), Anselmino L., Pavolini C., Saguí L., TortoRELLA ST. et TORTORICI E., 1981, Atlante delle forme ceramiche, I. Ceramica fine romana nel Bacino mediterraneo (medio e tardo impero), Enciclopedia dell'arte antica, Rome.

Carandini A. et Panella C. (dir.), 1977, Ostia IV. Le Terme del Nuotatore. Scavo dell'ambiente $X V I$ e dell'area $X X V$, Rome (Studi Miscellanei, 23).

Cavailles-Llopis M. et Vallatri L., 1994, Les céramiques fines importées, dans G. Demians d'Archimbaud G. (dir.), L'oppidum de Saint-Blaise du v' au vIF s. (Boucbes-duRbône), Paris (DAF 45), p. 86-115.

ChelBI F., 1992, Céramique à vernis noir de Cartbage, Tunis.

Cipriano M.T. et Carre M.-B., 1989, Production et typologie des amphores sur la côte adriatique de l'Italie, dans Ampbores romaines et bistoire économique, Actes du colloque (Sienne, 22-24 mai 1986), Rome, p. 67-104.

Cortr-MEzzoud Fl., 1998, Les fouilles de la Bourse (19671984). La vaisselle du sondage 10 , dans M. Bonifay, M.-B. Carre et Y. Rigoir, Fouilles à Marseille. Les mobiliers ( ${ }^{\text {er }}$ VIr' s.), Paris (Etudes Massaliètes 5), p. 137-161.

COMForT H., 1962, Late Ateius signatures, RCRFAcla, 4, p. 5-25.

COMFORT H., 1982, Signatures and Decoration on Italian and Gaulish Sigillata at Sabratha, $A / A, 86 / 4$, p. 483-507.
DaOllatli A., 1980, La céramique ifriqiyenne du $\mathrm{IX}^{\mathrm{c}}$ au $\mathrm{XV}^{\mathrm{e}}$ siècle, dans La céramique médiévale en Méditerranée occidentale $X^{e}-X V^{e}$ siècles, Actes du colloque (Valbonne, 1114 septembre 1978) (Colloques Internationaux du CNRS, $\mathrm{n}^{\circ}$ 584), Paris, p. 197-201.

DaOllatli A., 1995, La production vert et brun en Tunisie du $\mathrm{IX}^{\mathrm{e}}$ au XII ${ }^{\mathrm{e}}$ siècle. Étude historique et stylistique, dans Le Vert et le Brun, de Kairouan à Avignon, $X^{e}-X v^{e}$ siècle, Catalogue d'exposition (Marseille, La Vieille Charité, 1995), Marseille. p. 68-93.

DaOulatli A., 1995, La céramique médiévale en Tunisie. État de la recherche (IX'- $\mathrm{X}^{\mathrm{e}}$ siècles), Africa, XIII, p. 189-204.

DaOllatli A. et RammaH M., 1994, La céramique ifriqienne du ville au Xvie siècle, dans Couleurs de Tunisie, 25 siècles de céramique, Catalogue d'exposition (Institut du Monde Arabe, Paris - Musée des Augustins, Toulouse, 1994-1995). Paris, p. 83-181.

Deneauve J., 1972, Céramique et lampes africaines sur la côte de Provence, AntAfr, 6, p. 219-240.

Desbat A., Pernon J. et PiCON M., 1990, Terra sigillata lucente et productions des ateliers de Savoie : l'exemple d'Arles, dans PERNON J. et Chr., Les potiers de Portout, 1990, p. 183188.

Desbat A. et Picon M., 1994-95, Sur l'origine des céramiques sigillées claires B et luisantes du site du Bivan (38, L'Albenc), dans G. Ayala, Un lot de céramique du BasEmpire sur le site du Bivan (L'Albenc, Isère), $R A N, 27-28$, p. 267-268.

DORE J.N. avec la collaboration de SCHINKE R., 1992, First report on the pottery, dans N. Ben Lazreg et D. Mattingly, Leptiminus (Lamia), a Roman port city in Tunisia, Report $n^{\circ} 1$. Ann Arbor (JRA Suppl. No 4), p. 115-156.

DoRf J., 1989, The Coarse Pottery, dans J. Dore et N. Keay (dir.), Excavations at Sabratha 1948-1951. Vol. II. The finds, Tripoli, p. 87-248.

DORE J., 1996, The UNESCO Libyan Valleys Archaeological Survey Pottery, dans G. Barker, D. Gilbertson, B. Jones et D. Mattingly, Farming the Desert. The UNESCO Libyan Valleys Archaeological Survey, 2. Gazetter and Pottery. Londres, p. 318-389.

DORE J. et KEAY N. (dir.), 1989, Excavations at Sabratha 19481951. Vol. II. The finds, Tripoli.

Drine A., 1992-93, Note sur la ciste et le calathus de Chammakh (région de Zarzis), Africa, XI-XII, p. 147-157.

DrINE A., 1997, Muséographie. La réutilisation de l'église Notre Dame de la Garde de Zarzis en Musée, Africa, XV, p. 267-279.

Ettlinger E., Heidinger B., Hoffmain B. et al, 1990, Conspectus formarum terrae sigillatae italico modo confectae, Bonn. 
FENDRI M., 1961, Basiliques chrétiennes de la Skbira, Paris.

FENTREss L., 2001, Villas, wine and kilns : the landscape of Jerba in the late Hellenistic period, $J R A, 14, \mathrm{p}$ 249-268.

FÉVRIER P. A., 1963, Remarques préliminaires sur la céramique romaine d'Afrique du Nord. (À propos des fouilles de Sétif), RELig, XXIX, p. 125-136.

FÉVRIER P.-A., 1965, Fouilles de Sétif. Les basiliques chrétiennes du quartier nord-ouest, Paris.

Frova A. (dir.), 1977, Scavi di Luni, II, Relazione delle campagne di scavo 1972-1973-1974, Rome.

FULFORD M.G. et PEACOCK D.P.S., 1984, Excavations at Carthage: The British Mission, I, 2, The avenue du Président Habib Bourguiba, Salambo: The Pottery and other Ceramic Objects from the site, Sheffield.

Fulford M.G. et TimBY J., 1994, The fine wares, dans M.G. Fulford et D.P.S. Peacock (dir.), Excavations at Carthage, II, 2. The circular Harbour, North side, The pottery, Oxford.

FULFORD M. et TOMBER R., 1994, Excavations at Sabratha 19481951, II. The Finds, 2. The Finewares and Lamps, Londres (The Society for Libyan Studies Monograph $\mathrm{N}^{\circ} 3$ ).

GANDOLFI D., 1981, Terra sigillata chiara D di Albintimilium, RELig, XLVII, 1-4, p. 53-149.

GARABITO GOMEZ T., 1978, Los alfares romanos riojanos. Producción y commercialización, Madrid (Bibliotheca Praehistorica Hispana, 16).

Gelichi S. et Milanese M., 1997, Uchi Maius : la cittadella e il Foro, rapporto preliminare sulla campagna di scavo 1995 , dans M. Khanoussi et A. Mastino (dir.), Uchi maius I. Scavi e ricerche epigrafiche in Tunisia, Sassari, p. 49-94.

Ghalia T., Bonifay M. et CaPellu Cl., à paraître, L'atelier de Sidi-Zahruni : mise en evidence d'une production d'amphores de l'antiquité tardive sur le territoire de la cité de Neapolis (Nabeul, Tunisie), dans 1st Conference on Late Roman Coarse Wares, Cooking Wares and Amphorae in the Mediterranean : Archaeology and Archaeometry (Barcelone, 14-16 mars 2002), Oxford (BAR IS).

Golvin L., 1980, Les céramiques émaillées de période hammâdide Qal'a des Banû Hammâd (Algérie), dans $L a$ céramique médiévale en Méditerranée occidentale $X^{e}-X V^{e}$ siècles, Actes du colloque (Valbonne, 11-14 septembre 1978), Paris (Colloques Internationaux du CNRS, $n^{\circ} 584$ ), p. 203-217.

GOuRvest J. f, 1998, Introduction à l'étude des Terres Sigillées Claires, Orangées et Luisantes. Essai de classification du matériel des fouilles de Marseille, dans M. Bonifay, M.-B. Carre et Y. Rigoir, Fouilles à Marseille. Les mobiliers ( $F^{e r}$ $V I I^{e}$ s.), Paris (Études Massaliètes, 5), p. 19-52.
Guagliumi A. et Petriccione V., 1978, Roma in briciole, Antiqua, III, 8, p. 5-130.

GuÉRY R., 1971, Note sur trois vases de terra sigillata hispanique trouvés en Algérie, AntAfr, 5, p. 121-124.

GúÉRY R., 1979, Les marques de potiers sur terra sigillata découvertes en Algérie, I. Sigillées provinciales (hispanique et gallo-romaine), AntAfr, 13, p. 23-97.

GUÉRY R., 1982, L'importation de la terra sigillata gauloise en Afrique, RCRFActa XXI/XXII, p. 63-70.

GuÉRY R., 1984, L'occupation de Rougga (Bararus) d'après la stratigraphie du forum, BCTH, n.s., fasc.17 B, p. 7-99.

GuÉRY R., 1985, Survivance de la vie sédentaire pendant les invasions arabes en Tunisie centrale : l'exemple de Rougga, dans $I I^{e}$ Colloque International sur l'Histoire et l'Archéologie de l'Afrique du Nord (Grenoble, 5-9 avril 1983), BCTH, n.s., fasc. 19B, p 399-410.

GUÉRY R., 1992, Les marques de potiers sur terra sigillata découvertes en Algérie, IV/1. Sigillée italique (A( ) à $\mathrm{C}$. M( ) R( )), AntAfr, 28, p. 15-131

GUÉRY R., 1994, Les marques de potiers sur terra sigillata découvertes en Algérie, IV/2. Sigillée italique (ME( ) à $C$. VOLVSENVS (NESTOR ?)), AntAfr, 30, p. 89-187.

GUÉRY R. † et BONIFAY M., La céramique antique, dans M. Euzennat et H. Slim (dir.), Rougga, I. Le forum et ses abords. (à paraître).

HAYES J.W., 1972, Late Roman Pottery, Londres.

HAYES J.W., 1973, Roman pottery from the South Stoa at Corinth, Hesperia, 42, p. 416-470.

HAYES J.W., 1976, Pottery : Stratified Groups and Typology, dans J.H. Humphrey (dir.), Excavations at Carthage 1975 conducted by the University of Michigan, I, Tunis, p. 47123.

HAYES J.W., 1978, Pottery Report 1976, dans J.H. Humphrey (dir.), Excavations at Carthage 1976 conducted by the University of Michigan, IV, Ann Arbor, p. 23-98.

Hedinger B., von Schnurbein S., Schneider G., 1999, Die Frühe Terra Sigillata aus den Grabungen des Deutschen Archäologischen Instituts in Karthago 1974-1991, dans F. Rakob (dir.), Karthago III, Mayence, p. 289-402.

Jaritz H., Favre S., Nogara G., Rodziewicz M. et Carrfz-MaraTRAY J.-Y., 1994, Pelusium. Prospection archéologique et topographique de la région de Kana'is, CRIPEL, 16, p. 123166.

Jodin A. et Ponsich M., 1960, La céramique estampée du Maroc romain, $B A M, \mathrm{IV}$, p. $285-318$. 
KENRICK Ph.M., 1985, Excavations at Sidi Khrabish, Benghazi (Berenice), III, 1, The Fine Pottery, Tripoli (Libya Antiqua, Suppl. V).

KENRICK Ph.M., 1996, The importation of italian sigillata to Algeria, AntAfr, 32, p. 37-44.

KenRICK Ph.M., 1997, Cn. Ateius, the inside story, RCRFActa, 35 . p. $179-190$.

LAMBOGLIA N., 1952, Per una classificazione preliminare della ceramica campana, dans Atti del primo congresso di studi liguri, 1950, Bordighera, p. 139-206.

LAmboglia N., 1958, Nuove osservazioni sulla terra sigillata chiara, I. Tipi A e B, RELig, XXIV, p. 257-330.

LAmboglia N., 1963, Nuove osservazioni sulla terra sigillata chiara, II. Tipi C, Lucente e D, RELig, XXIX, p. 145-212.

Lamboglia N., 1964, La campagna 1963 sul relitto di Punta Scaletta all'isola di Giannutri (relazione preliminare), RELig, XXX, p. 229-257.

LANDLRÉ C. et PASQualini M., 1997, Evolution de l'occupation humaine et de l'environnement dans le delta du Rhône. Nouvelles perpectives de recherches, dans Crau, Alpilles, Camargue. Histoire et archéologie. Actes du colloque (Arles, 18-19 novembre 1995), Arles, p. 7-11.

LAPORTE J.-M., 1981, La céramique sigillée de Lescar (Benebarnum). Fouilles 1976-1980, T.E.R., Université de Pau et des Pays de l'Adour.

LAUMONIER A., 1997, La céramique bellénistique à reliefs, 1, Ateliers ioniens, Paris (Explorations archéologiques à Délos, 31).

LAVOIF C., 1989, Les traces d'ateliers de poterie à Sullecthum, dans Sullectbum I, CEA, XXII, p. 91-109.

LECIEJEWICZ L., TABACZYNSKa E. et TabaCZYNSKi St., 1977, Torcello. Scavi 1961-62, Rome.

LouHICHI A., 1992-93, La céramique de l'lle de Tabarka : étude préliminaire, Africa, XI-XII, p. 89-108.

LOUHICHI A., 1992-93, Un échantillonnage de céramique d'époque médiévale de Qairawan. Exemple d'application de recherches archéologiques et de laboratoire, Africa, XIXII, p. 258-276.

LouniCH A., 1997, La céramique fatimide et ziricle de Madhia d'après les fouilles de Qars al-Qaïm, dans La céramique médiévale en Méditerranée. Actes du VI' congrès de l'AIECM2 (Aix-en-Provence, 13-18 novembre 1995), Aix-enProvence, p. 301-310.

Lounichi A., 1998, La céramique islamique de Dougga, Africa, XVI, p. 109-127.
LOLHICHI A. et PICON M., 1983, Importation de matériel céramique ifriqiyen en Mauritanie, Revue d'Archéométrie, 7 , p. $45-58$.

LUND J., 1995, Hellenistic, Roman and Late Roman Fine Wares from the Segermes Valley - Forms and Chronology, dans S. Dietz, L. Ladjimi Sebaï et H. Ben Hassen, Africa Proconsularis, Regional Studies in the Segermes Valley of Northern. Tunesia, II, Copenhague, p. 449-629.

MACKENSEN M., 1993, Die spätantiken Sigillata- und Lampentöpfereien von El Mabrine (Nordtunesien), Münich (Münchner Beiträge zur Vor- und Frühgeschichte, Band $50)$.

MANNONI T., 1972, Analisi mineralogiche delle ceramiche mediterranee. Nota II, dans Atti del V Convegno Internazionale della Ceramica, Albisola, p. 107-128.

MANNONI T., 1979, Analisi mineralogiche delle ceramiche mediterranee. Nota VI, dans Atti del XII Convegno Internazionale della Ceramica, Albisola, p. 229-239.

MaNNONi T., 1997, Una precisazione sul quarzo eolico, dans Atti del Convegno: Il contributo delle analisi archeometriche allo studio delle ceramiche grezze e comuni, Bologna, p. 128.

Martin A.N., 1996, Altre sigillate, dans R. Volpe (dir.), Aqua Marcia, Lo scavo di un tratto urbano, Florence, p. 126.

MARTIN Th., 2002, Un vase en terre sigillée hispanique trouvé au Mas-d'Agenais (Lot-et-Garonne), dans M. Genin, A. Vernhet (dir.), Céramiques de la Graufesenque et autres productions d'époque romaine, Nouvelles recherches, Hommages à Bettina Hoffmann, Montagnac (Archéologie et Histoire Romaine, 7), p. 289-294.

MARTIN-KILCHER S., Karthago 1999, Die Füllung eines frühkaiserzeitlichen Pozzo, dans F. Rakob (dir.), Karthago III, Mayence, p. 403-434.

MARTINEZ Mujilla C., 1950, Vaso de " terra sigillata "hispanica hallado en Marsella, AEArq, 23, p. 210-211.

MAYET Fr., 1969, Expansion de la céramique sigillée hispanique en Aquitaine, $M C V, 5$, p. 73-101.

MAYET Fr., 1984. Les céramiques sigillées hispaniques. Contribution à l'bistoire économique de la péninsule Ibérique sous l'Empire Romain, Bordeaux.

MEzQuíriz dF Catalan M.A., 1960, Aportaciones al estudio de la expansión de la sigillata hispánica en el sur de Francia, AEArq, 28, p. 210-214.

MEzQtíriz dD: Catal.AN M.A., 1961, Terra Sigillata Hispanica, Valence.

MEzQuíkIz M.A., 1985, Terra Sigillata Ispanica, dans Atlante delle forme ceramicbe, II. Ceramica fine romana nel bacino mediterraneo (tardo ellenismo e primo impero), 
Rome (Enciclopedia dell'Arte Antica, Classica e Orientale), p. 97-174 et pl. XXIV à LIV.

MOKRANi M.-A., 1997, À propos des céramiques récemment découvertes lors des fouilles sur le site d'Achir ( $\mathrm{X}^{\mathrm{e}}-\mathrm{XI}^{\mathrm{c}}$ siècles), dans La céramique médiévale en Méditerranée. Actes du VI* congrès de l'AIECM2 (Aix-en-Provence, 13-18 novembre 1995), Aix-en-Provence, p. 291-300.

MOREL J.-P., 1981, La céramique campanienne : les formes, Rome.

MOREl J.-P., 1982, La céramique à vernis noir de CarthageByrsa : nouvelles données et éléments de comparaison, dans Actes du colloque sur la céramique antique (Carthage, 23-24 juin 1980), Carthage (CEDAC, Dossier 1), p. 43-76.

MOREL J.-P., 1986, La céramique à vernis noir de Carthage, sa diffusion, son influence, dans Carthage VIII, CEA, XVIII, p. $25-68$.

Mouchot D., 1970, Épave romaine "A " du Port de Monaco, Bulletin du Musée d'Anthropologie Prébistorique de Monaco, 15, p. 159-201.

Neuru L., 1986, Cooking wares of the byzantine epoch at Carthage : some observations, dans Carthage VIII, CEA, XVIII, p. 69-79.

NEURU L., 1987, Red slipped wares of southwestern central Tunisia : new evidence, RCRFActa, XXV/XXVI, p. 175-188.

OpaIT A., 1998, The north african Dressel 1, dans El vi a l'antiguitat. Economia, producció $i$ commerç al mediterrani oocidental, Actes du 2 e colloque international d'archéologie romaine (Badalone, 6-9 mai 1998), Badalone, Musée de Badalone (Monografies Badalonines, num. 14), p. 427429.

OpAIT A., Early Roman Amphorae from Leptiminus, RCRFActa, 36, p. 439-442.

OXE A. et COMfort H., 1968, Corpus vasorum arretinorum. A Catalogue of the Signatures, Shapes and Chronology of Italian Sigillata, Bonn.

Pallakès Fr., 1963, Terra sigillata ispanica ad Ostia, RELig, XXIX, p. 69-82.

Panella Cl., 1973, Le anfore, dans A. Carandini et C. Panella (dir.), Ostia III, Rome (Studi Miscellanei, 21), p. 463-633.

Pareja F., 1972, Marques sur une amphore de l'île de Fraile (Aguilas), Cabiers d'Archéologie Subaquatique, I, p $107-$ 108.

PAvouini C., 1998, Le lucerne in Italia nel VI-VII secolo d.C. : alcuni contesti significativi, dans L. Saguí (dir.), Ceramica in Italia: VI-VII secolo, Atti del Convegno in onore di John W. Hayes (Roma, 11-12 maggio 1995), Florence, p. 123140.
PEACOCK D.P.S., 1984, Petrology and origins, dans M.G. Fulford et D.P.S. Peacock, Excavations at Carthage: The British Mission, I, 2, The avenue du Président Habib Bourguiba, Salambo: The Pottery and other Ceramic Objects from the site, Sheffield, pp. 6-20.

PEACOCK D.P.S., BÉJAOUI F. et BEN LAZREG N., 1989, Roman amphora production in the Sahel region of Tunisia, dans Amphores romaines et bistoire économique, Actes du colloque (Sienne, 22-24 mai 1986), Rome, p. 179-222.

PEacock D.P.S., BÉJaOUi F. et Bfo Lazreg N., 1990, Roman pottery production in central Tunisia, $J R A, 3$, p. 59-84.

PEACOCK D.P.S. et TOMBER R. 1991, Roman amphoras kilns in the Sahel of Tunisia : petrographic investigation of kiln material from sedimentary environment, dans A. Middleton et I. Freestone (dir.), Recent developments in ceramic petro$\log y$, Londres (British Museum Occasional Papers, 81), p. 289-304.

PfRNon J. et Chr., 1990, Les potiers de Portout, Productions, activités et cadre de vie d'un atelier au ves. ap. J.-C. en Savoie, Paris (RAN, Supplément 20).

PIERI D., 1998, Les importations d'amphores orientales en Gaule méridionale durant l'Antiquité tardive et le haut Moyen-Age (IVe - VII s. ap. J.-C.). Typologie, chronologie et contenu, dans SFECAG, Actes du Congrès d'Istres, Marseille, p. 97-106.

Picon M. et Desbat A., 1986, Sigillée claire B et luisante : classification et provenance, Figlina, 7, p. 5-18.

PRÖTlEL Ph.M., 1996, Mediterrane Feinkeramikimporte des 2. bis 7. Jabrbunderts N.Chr. im Oberen Adriaraum und in Slovenien, Cologne (Kölner Studien zur Archäologie der Römischen Provinzen, Band 2).

RAMON TORRÈs J., 1995, Las ánforas fenicio-puinicas del Mediterráneo central y occidental, Barcelone (Instrumenta, 2).

Remola I Vallverdú J.A. et Abello i Riley A., 1989, les àmfores, dans TED'A, Un abocador del segle VD.C. en el forum provincial de Tarraco, Tarragone, p. 249-320.

REYNOLDS P., 1995, Trade in the Western Mediterranean AD 400-700: The Ceramic Evidence, Oxford (BAR Intern. Series, 604).

RiGOIR J., 1968, Les sigillées paléochrétiennes grises et orangées, Gallia, p. 177-244.

RigolR Y. et J., 1987, Des dérivées-des-sigillées paléochrétiennes, dans Céramiques bellénistiques et romaines II, Besançon, p. 329-338.

RILEY J.A., 1981, The pottery from the cistern 1977.1, 1977.2 and 1977.3, dans J.H. Humphrey (dir.), Excavations at Cartbage conducted by the University of Michigan VI, Ann Arbor, p. 86-124. 
Ru.EY J.A., 1982, Islamic Ware from Ajdabiyah, Libyan Studies, 13 , p. $85-104$.

ROCa Rocye..s M., 1976, Sigillala bispánica producida en Anduijar (Jaén), Jaén.

SABRIÉ R., 1981, Sigillée hispanique, dans Y. Solier (dir.), Les épaves de Gruissan, Archaeonautica, 3, p. 7-264 (p. $247-$ 249).

SAGLì L., 1980, Ceramica africana dalla "villa di Tiberio "a Sperlonga, MEFRA, 92, p. 471-544.

Salomorson J.W., 1968, Étude sur la céramique romaine d'Afrique sigillée claire et céramique commune de Henchir el Ouiba (Raqqada) en Tunisie centrale, BABesch, XLIII, p. $80-145$.

SALOMONSON J.W., 1969, Spätrömische rote Tonware mit Reliefverzierung aus Nordafrikanischen Werkstätten. Entwicklungsgeschitliche Untersuchungen zur relief geschmückten Terra Sigillata Chiara C, BABesch, XLIV, p. 4-109.

Savtamaria Cl., 1995, Lépave Dramont Eà Saint Raphaël (ves. ap.J.-C.), Paris (Archaeonautica, 13).

Schuring J.M., 1988, Terra Sigillata Africana from the San Sisto Vecchio in Rome, BABesch, 63, p. 1-68.

SIEBBERT G., 1980, Les bols à reliefs, une industrie d'art de l'époque hellénistique, dans Céramiques bellénistiques et romaines, Besançon (An. Lit. Univ. Besançon, 242), p. 5583.

Sim L., Bonifay M., Trolssft P., avec Blanc-Bijon V., Foy D., Gassend J.-M., Hallier G., PAskoff R., Pasqlalin M., PITON J. et Stiknibirg M., 1999, L'usine de salaison de Neapolis (Nabeul). Premiers résultats des fouilles 1995-1998, Africa, XVII, p. 153-197.

SOREN D., 1978, The roman pottery from Utica (Tunisia), CEA, VIII, p. 109-141.

Soricflu. G., 1994, Terra Sigillata della prima, media e tarda età imperiale, clans P. Arthur (dir.), Il complesso Arcbeologio di Carminiello ai Mannesi, Napoli (Scavi 1983-1984), Naples, p. 109-168.

SOTOMAYOR M., 1977, Marcas y estilos en la sigillata decorada de Andịjar, Jaén.
SPARKES B. et TALCOTT L., 1970, Black and plain pottery of the 6th, 5th and 4th centuries B.C., Princeton (The Athenian Agora XII, 1 et 2).

STER: E.M., 1968, Note analytique sur des tessons de sigillée claire ramassés à Henchir es Srira et Sidi Aïch, BABeschb, 43. p. $147-154$.

StONe D.L., STIRl.ING L.M. et Bex LazkF; N., 1998, Suburban land-use and ceramic production around Leptiminus (Tunisia) : interim report, $J R A, 11$, p. 304-317.

TINBBY J.R., 1994, Red Slipped wares, dans M. Fulford et R. Tomber (dir.), Excavations at Sabratha 1948-1951, II. The Finds, 2. The Finewares and Lamps, Londres (The Society for Libyan Studies Monograph No. 3), p. 67-118.

Treglia J.-C., 1998, Les fouilles de la Bourse (1967-1984). La vaisselle des sondages 11 et 12 , dans M. Bonifay, M.-B. Carre et Y. Rigoir, Fouilles à Marseille. Les mobiliers ( ${ }^{o r}{ }_{-}$ $V I r^{\prime}$ s.), Paris (Études Massaliètes, 5), p. 175-189.

VAlLALRI L., 1997, Les céramiques importées, dans H. Marchesi, J. Thiriot et L. Vallauri (dir.), avec la collaboration de M. Leenhardt, Marseille, les ateliers de poliers du XIIr' siècle et le quartier Sainte-Barbe (V'-XVIF' S.), Paris (DAF 65), p. 79-92.

VallajRI L., Les céramiques d'époque islamique, dans M. Euzennat et H. Slim (dir.), Rougga, I. Le forum et ses abords, à paraître.

VAnderhoeven M., 1988, La terre sigillée. Campagnes de fouille de 1976 à 1986, dans J. Mertens (dir.), Ordona VIII. Bruxelles-Rome, p. 85-218.

VAN DER WERFF J.H., 1982, Uzita, Matériel de fouilles d'une cité antique en Tunisie centrale, Utrecht.

Vegas M., 1994, La céramique du camp à Simitthus, dans M. Khanoussi et al., Simitlbus II. Der Tempelberg und das römische Lager, Mayence, p. 141-244.

VIRTET H., 1961, Ceramica romana imperial hallada en Oued Bibi (Constantina, Argelia), AEArq, 34, p. 204-206.

VITEu, G., 1981. Islamic Carthage. The archaeological, bistorical and ceramic evidence, Carthage (CEDAC, dossier $\mathrm{n}^{\circ} 2$ ).

WAAGí F.O., 1948, Hellenistic and Roman Tableware of North Syria, dans Antioch-on-the-Orontes IV, 1, Ceramics and Islamic Coins, Princeton, p. 1-60. 


\section{INDEX GÉOGRAPHIQUE}

\section{A - ORDRE DU CATALOGUE}

1 - Henchir el Mdeina

2 - Solb ech Chergui

3 - Solb ech Chergui ouest

4 - Henchir Daoui

5 - Henchir bou Gornine

6 - Bir Ouled Mohammed

7 - Rass Zarba

8 - Sidi Mohammed Chraouch

9 - Fesguiet Jdaria - Solb el Gharbi

10 - Henchir Fesguia Rouis

11 - Henchir Chaabane

12 - Henchir el Abid

12 bis - Naoura : site

13 - Sidi bou Teffaha

14 - Oued Ogla - Oued Ennouili

15 - Lalla Meriem

16 - El Kantara

16 bis - El Gastil

17 - Sidi Garous

18 - Lalla Hadria (ou Tobkhana)

19 - Rhizene

20 - Cheikh Yaya

21 - Guellala

22 - Ersifet

23 - Rass Segala

24 - Henchir Karbub

25 - Port de Gigthis

26 - Oued Babous est

27 - Bir Tajerimet

28 - Bir Slama est

29 - Henchir Chelakhi

30 - Henchir Daous

31 - Laflala (Hir Roumia ou Fastaqia)

32 - Gourine est

33 - Henchir bou Amia

34 - Henchir Medina (Zarat est)

35 - Oued Zerkine

36 - Et Tarfaia

37 - Henchir Chougaf
38 - Henchir er Rekhama

39 - Oued el Akarit sud

40 - Henchir Adame

41 - Sebkhet el Guettiate

42 - En Nadour

43 - Bir Flaguess-TRAPSA

44 - Henchir Ferchatt

45 - Zabouza

46 - Rass el Ferchatt

47 - Henchir Jel

48 - Oued Maltine

49 - El Jaziret el Rharbia

50 - Dzirat el Laboua

51 - Dzirat el Hajar

52 - Dzirat el Bessila

53 - El Khefifia

54 - Onga

55 - Henchir Leich

56 - Thyna

57 - El Guedaia

58 - Rjel ech Chouggaf

59 - Rass el Berghout ouest

60 - Rass el Berghout est

61 - Borj el Hassar

62 - El Abassia

63 - Gremdi

64 - Rass bou Nouma

65 - Sefnou

66 - Enf er Rkik est

67 - Enf er Rkik ouest

68 - Henchir ech Cheggaf - Sidi Mansour

69 - Nadour Sidi Mansour

70 - El Aouabed - Koudiat Jaber

71 - Henchir el Majdoul

72 - Ksar en Nouba

73 - Henchir el Flouss

74 - Sidi el Khafi

75 - Sidi Mezara sud

76 - Sicli Mezara nord

77 - La Louza I sud

78 - La Louza II nord
79 - Sidi Saad

80 - La Louata

81 - Rass bou Tria

82 - Ouled Slim

83 - Ouled Mabrouk

84 - Marsa Melloulèche

85 - Rass Jezira

86 - Jezira

87 - Tour Khedija

88 - Sidi Abdallah el Merrakchi

89 - Maqluba

90 - El Alia - Sebkha Njila

91 - Borj el Mzaouak

92 - Henchir el Mzaouak

93 - Ghar el Dheba

94 - Salakta sud

95 - Rass Salakta

96 - Mnaka sud

97 - Mnaka nord

98 - Douira sud

99 - Douira nord

100 - Rejiche

101 - Sidi ben Ghayada

102 - Mahdia port ancien

103 - Mahdia nécropole

104 - El Hafsi - Neyret

105 - Thapsus - Rass Dimass

106 - Ech Chott

107 - Ras el Ain

108 - Port Soukrine est

109 - Sayada est

110 - Lamta

111 - Mansourah est

112 - Mansourah ouest

113 - Sidi Mansour

114 - Grande Kuriate

115 - Al Shrir

116 - El Ghedamsi

117 - Sebkha Skanes

118 - Chott Meryem

119 - Halk el Mujien sud

120 - Halk el Mujien nord 
121 - Hergla sud

122 - Hergla centre

123 - Hergla nord

124 - Borj el Medfoun

125 - Borj el Assa el Jiriba

126 - Cote 14

127 - Bou Kesra

127 bis - Sebkha Sidi Khalifa

128 - Henchir el Kebir

129 - Henchir el Hefair

130 - Henchir Selloum

131 - Bir ech Choukaf

132 - Pupput

133 - Bit el Assa

134 - Sidi el Mehersi

135 - Nabeul

136 - Sidi Ameur bou Chouika

137 - Maamoura

138 - Khlij - Ksir el Rhalem

139 - Htouba

140 - Sidi Mosba - Dar ben Slimane

141 - Sidi Othmane

142 - Dar el Kbira

143 - Cote 28 - Seba Argoud

144 - Gasser Saad - Sebka Lebna

145 - Cote 12 - Henchir Lebna

146 - Ain Sghira

147 - Sidi Ali Moujehed sud

148 - Sidi Ali Moujehed nord

149 - Sebkhet Tafekhsit

150 - Kelibia

151 - Demna

151 bis - Oued el Kseub
152 - Es Seguia sud

153 - Es Seguia nord

154 - Kerkouane

155 - El Hannaker

156 - Ain lakerdouch

157 - Rass ed Derek - Oued er Rega

158 - El Haouaria

159 - Rhirane bou Mliha

160 - Borj Guelib el Mdaoueur

161 - Sidi Daoud - Corps de garde

161 bis - Sidi Daoud - Djilia

162 - Sidi Daoud - Misua

163 - Sidi Daoud - Carrières

164 - Degla

165 - Mrä̈ssa

166 - El Assa est

167 - El Assa - cote 9

168 - Marsa ben Ramdan

169 - Sidi Raïs

170 - Carthage

171 - Gammarth - Le Pêcheur

171 bis - Cap Gammarth

172 - Raouad

173 - Sebkha Ariana

174 - Utique

175 - Ras et Tarf

176 - Ain Demna est

177 - Ain Demna

178 - Raf-Raf - Dar el Khadar

179 - Sounine

180 - Ras Jebel - El Hadouba

181 - Safi

\section{B - CLASSEMENT ALPHABÉTIQUE}

Ain bou Thouir : site $n^{\circ} 186$

Ain Demna : site $n^{\circ} 177$

Ain Demna est : site $n^{\circ} 176$

Ain el Merja : site $n^{\circ} 183$

Ain Sghira : site $n^{\circ} 146$

Ain Takerdouch : site $n^{\circ} 156$

Al Shrir : site $n^{\circ} 115$

Argoub el Bania : site $n^{\circ} 208$

Bir ech Choukaf : site no 131

Bir Flaguess - TRAPSA : site $n^{\circ} 43$

Bir Ouled Mohammed : site $n^{\circ} 6$
Bir Slama est : site $\mathrm{n}^{\circ} 28$

Bir Tajerjimet : site $n^{\circ} 27$

Bit el Assa : site $n^{\circ} 133$

Borj el Assa el Jiriba : site $n^{\circ} 125$

Borj el Hassar : site $n^{\circ} 61$

Borj el Medfoun : site $n^{\circ} 124$

Borj el Mzaouak : site $n^{\circ} 91$

Borj Guelib el Mdaoueur : site $n^{\circ} 160$

Bou Kesra : site $n^{\circ} 127$

Cap Gammarth : site $n^{\circ} 171$ his
182 - Rass ez Zebib

183 - Ain el Merja

184 - R'mel

185 - Henchir Chaara

186 - Ain bou Thouir

187 - Pointe el Ouali

188 - Gouraya

189 - Henchir Rara

189 bis - Jebel Nador

190 - Oued ed Damous

190 bis - Henchir es Sahel I

191 - Henchir es Sahel II

192 - Henchir es Sahel III

193 - Henchir es Sahel IV

194 - Henchir es Sahel V

195 - Dar Chraiba (Dar Hassaina)

196 - Rass Ghirane (Rass ben Sekka)

197 - Rass Enghela - cote 1

198 - Rass Enghela est

199 - Rass Enghela ouest

200 - Khechad - cote 9

201 - Vigie Rass el Koran est

202 - Vigie Rass el Koran

203 - Rass el Koran

203 bis - Sidi Béchir

204 - Marsa Douiba

205 - Sidi Gherib

206 - Sidi Mechrig

207 - Oum en Noual

208 - Argoub el Bania

209 - Tabarka

201 - La Galite - L'Escueil de Paque
Carthage : site $n^{\circ} 170$

Cheikh Yaya : site $n^{\circ} 20$

Chott Meryem : site $n^{\circ} 118$

Cote 12 - Henchir Lebna : site $\mathrm{n}^{\circ} 145$

Cote 14 : site $n^{\circ} 126$

Cote 28 - Seba Argoud : site n 143

Darben Slimane - Sidi Mosba : site $n^{\circ} 140$

Dar Chraiba (Dar Hassaina) : site $\mathrm{n}^{\circ} 195$ 
Dar el Kbira : site $n^{\circ} 142$

Dar el Khadar - Raf-Raf : site $\mathrm{n}^{\circ} 178$

Degla : site $\mathrm{n}^{\circ} 164$

Demna : site $n^{\circ} 151$

Djilia - Sidi Daoud : site $n^{\circ} 161$ bis

Douira nord : site $n^{\circ} 99$

Douira sud : site $n^{\circ} 98$

Dzirat el Bessila : site $\mathrm{n}^{\circ} 52$

Dzirat el Hajar : site $n^{\circ} 51$

Dzirat el Laboua : site $n^{\circ} 50$

Ech Chott : site $n^{\circ} 106$

El Abassia : site $n^{\circ} 62$

El Alia - Sebkha Njila : site $n^{\circ} 90$

El Aouabed - Koudiat Jaber : site $n^{\circ} 70$

El Assa - cote 9 : site $n^{\circ} 167$

El Assa est : site $n^{\circ} 166$

El Gastil : site $n^{\circ} 16$ bis

El Ghedamsi : site $n^{\circ} 116$

El Guedaia : site $n^{\circ} 57$

El Hadouba - Ras Jebel : site $\mathrm{n}^{\circ} 180$

El Hafsi - Neyret : site $n^{\circ} 104$

El Hannaker : site $n^{\circ} 155$

El Haouaria : site $n^{\circ} 158$

El Jaziret el Rharbia : site $n^{\circ} 49$

El Kantara : site $n^{\circ} 16$

El Khefifia : site $n^{\circ} 53$

En Nadour : site $n^{\circ} 42$

Enf er Rkik est : site $n^{\circ} 66$

Enf er Rkik ouest : site $n^{\circ} 67$

Ersifet : site $n^{\circ} 22$

Es Seguia nord : site $n^{\circ} 153$

Es Seguia sud : site $n^{\circ} 152$

Et Tarfaia : site $n^{\circ} 36$

Fastaqia : site $n^{\circ} 31$

Fesguiet Jdaria - Solb el Gharbi : site $n^{\circ} 9$

Gammarth (cap) : site $n^{\circ} 171$ bis

Gammarth - Le Pêcheur : site $n^{\circ} 171$

Gasser Saad - Sebka Lebna : site $n^{\circ} 144$

Ghar el Dheba : site $n^{\circ} 93$

Gouraya : site $n^{\circ} 188$

Gourine est : site $n^{\circ} 32$

Grande Kuriate : site $\mathrm{n}^{\circ} 114$
Gremdi : site $n^{\circ} 63$

Guellala : site $n^{\circ} 21$

Halk el Mujjen nord : site $n^{\circ} 120$

Halk el Muijen sud : site $n^{\circ} 119$

Henchir Adame : sile $n^{\circ} 40$

Henchir bou Amia : site $n^{\circ} 33$

Henchir bou Gornine : site $n^{\circ} 5$

Henchir Chaabane : site $n^{\circ} 11$

Henchir Chaara : site $n^{\circ} 185$

Henchir Chelakhi : site $n^{\circ} 29$

Henchir Chougaf : site $n^{\circ} 37$

Henchir Daoui : site $n^{\circ} 4$

Henchir Daous : site $n^{\circ} 30$

Henchir ech Cheggaf - Sidi

Mansour : site $n^{\circ} 68$

Henchir el Abid : site $n^{\circ} 12$

Henchir el Flouss : site $n^{\circ} 73$

Henchir el Hefair : site $n^{\circ} 129$

Henchir el Kebir : site $n^{\circ} 128$

Henchir el Majdoul : site $n^{\circ} 71$

Henchir el Mdeina : site $n^{\circ} 1$

Henchir el Mzaouak : site $n^{\circ} 92$

Henchir er Rekhama : site $n^{\circ} 38$

Henchir es Sahel I : site $n^{\circ} 190$ bis

Henchir es Sahel II : site $n^{\circ} 191$

Henchir es Sahel III : site ${ }^{\circ} 192$

Henchir es Sahel IV : site $n^{\circ} 193$

Henchir es Sahel V : site $n^{\circ} 194$

Henchir Ferchatt : site $n^{\circ} 44$

Henchir Fesguia Rouis : site $n^{\circ} 10$

Henchir Jel : site $n^{\circ} 47$

Henchir Karbub : site $n^{\circ} 24$

Henchir Lebna : site $n^{\circ} 145$

Henchir Leich : site $n^{\circ} 55$

Henchir Medina (Zarat est) : site $\mathrm{n}^{\circ} 34$

Henchir Rara : site $n^{\circ} 189$

Henchir Roumia : site $n^{\circ} 31$

Henchir Selloum : site $n^{\circ} 130$

Hergla centre : site $n^{\circ} 122$

Hergla nord : site $n^{\circ} 123$

Hergla sud : site $n^{\circ} 121$

Htouba : site $\mathrm{n}^{\circ} 139$

Port de Gigthis : site $\mathrm{n}^{\circ} 25$

Jebel Nador : site $n^{\circ} 189$ bis

Jezira : site $n^{\circ} 86$

Kelibia : site $n^{\circ} 150$

Kerkouane : site $n^{\circ} 154$
Khechad - cote 9: site $n^{\circ} 200$

Khlij - Ksir el Rhalem : site $n^{\circ} 138$

Koudiat Jaber - El Aouabed : site $n^{\circ} 70$

Ksar en Nouba : site $n^{\circ} 72$

Kuriate (Grande) : site $\mathrm{n}^{\circ} 114$

La Galite - L'Escueil de Paque : site $\mathrm{n}^{\circ} 210$

La Louata : site $n^{\circ} 80$

La Louza I sud : site $n^{\circ} 77$

La Louza II nord : site $n^{\circ} 78$

Laflala (Hir Roumia ou Fastaqia) : site $n^{\circ} 31$

Lalla Hadria (ou Tobkhana) : site $\mathrm{n}^{\circ} 18$

Lalla Meriem : site $n^{\circ} 15$

Lamta : site $n^{\circ} 110$

Maamoura : site $n^{\circ} 137$

Mahdia nécropole : site $\mathrm{n}^{\circ} 103$

Mahdia port ancien : site $n^{\circ} 102$

Mansourah est : site $n^{\circ} 111$

Mansourah ouest : site $n^{\circ} 112$

Maqluba : site $n^{\circ} 89$

Marsa ben Ramdan : site $n^{\circ} 168$

Marsa Douiba : site $n^{\circ} 204$

Marsa Melloulèche : site $n^{\circ} 84$

Misua - Sidi Daoud : site ${ }^{\circ} 162$

Mnaka nord : site $n^{\circ} 97$

Mnaka sud : site $n^{\circ} 96$

Mraïssa : site $n^{\circ} 165$

Nabeul : site $\mathrm{n}^{\circ} 135$

Nadour Sidi Mansour : site $n^{\circ} 69$

Naoura : site $\mathrm{n}^{\circ} 12$ bis

Neyret - El Hafsi : site $n^{\circ} 104$

Onga : site $\mathrm{n}^{\circ} 54$

Oued Babous est : site $n^{\circ} 26$

Oued ed Damous : site $n^{\circ} 190$

Oued el Akarit sud : site $n^{\circ} 39$

Oued el Kseub : site $n^{\circ} 151$ bis

Oued er Rega- Rass ed Derek : site $n^{\circ} 157$

Oued Maltine : site $n^{\circ} 48$

Oued Ogla - Oued Ennouili : site $n^{\circ} 14$

Oued Zerkine : site ${ }^{\circ} 35$

Ouled Mabrouk : site $\mathrm{n}^{\circ} 83$

Ouled Slim : site $\mathrm{n}^{\circ} 82$

Oum en Noual : site $n^{\circ} 207$ 
Pointe el Ouali : site $\mathrm{n}^{\circ} 187$

Port Soukrine est : site $n^{\circ} 108$

Pupput : site $\mathrm{n}^{\circ} 132$

R'mel : site $n^{\circ} 184$

Raf-Raf - Dar el Khadar : site $\mathrm{n}^{\circ} 178$

Raouad : site $\mathrm{n}^{\circ} 172$

Ras el Ain : site $n^{\circ} 107$

Ras et Tarf : site $n^{\circ} 175$

Ras Jebel - El Hadouba : site $\mathrm{n}^{\circ} 180$

Rass bou Nouma : site $n^{\circ} 64$

Rass bou Tria : site $n^{\circ} 81$

Rass Dimass - Thapsus : site $\mathrm{n}^{\circ} 105$

Rass ed Derek - Oued er Rega : site $n^{\circ} 157$

Rass el Berghout est : site $n^{\circ} 60$

Rass el Berghout ouest : site $n^{\circ} 59$

Rass el Ferchatt : site $n^{\circ} 46$

Rass el Koran : site $n^{\circ} 203$

Rass el Koran (Vigie) : sites $\mathrm{n}^{\mathrm{os}} 201-202$

Rass Enghela - cote 1 : site ${ }^{\circ} 197$

Rass Enghela est : site $n^{\circ} 198$

Rass Enghela ouest : site $n^{\circ} 199$

Rass ez Zebib : site $n^{\circ} 182$

Rass Ghirane (Rass ben Sekka) : site $n^{\circ} 196$

Rass Jezira : site $n^{\circ} 85$

Rass Salakta : site $n^{\circ} 95$

Rass Segala : site $n^{\circ} 23$

Rass Zarba : site $n^{\circ} 7$
Rejiche : site $n^{\circ} 100$

Rhirane bou Mliha : site $n^{\circ} 159$

Rhizene : site $\mathrm{n}^{\circ} 19$

Rjel ech Chouggaf : site $n^{\circ} 58$

Safi : site $n^{\circ} 181$

Salakta sud : site $n^{\circ} 94$

Sayada est : site $n^{\circ} 109$

Seba Argoub : site $n^{\circ} 145$

Sebkha Ariana : site $n^{\circ} 173$

Sebkha Lebna - Gasser Saad : site $n^{\circ} 144$

Sebkha Njila - El Alia : site $n^{\circ} 90$

Sebkha Sidi Khalifa : site $n^{\circ} 127$ bis

Sebkha Skanes : site $n^{\circ} 117$

Sebkhet el Guettiate : site $n^{\circ} 41$

Sebkhet Tafekhsit : site $\mathrm{n}^{\circ} 149$

Sefnou : site $n^{\circ} 65$

Sidi Abdallah el Merrakchi : site $n^{\circ} 88$

Sidi Ali Moujehed nord : site $n^{\circ} 148$

Sidi Ali Moujehed sud : site $n^{\circ} 147$

Sidi Ameur bou Chouika : site $n^{\circ} 136$

Sidi Béchir : site $n^{\circ} 203$ bis

Sidi ben Ghayada : site $\mathrm{n}^{\circ} 101$

Sidi bou Teffaha : site $n^{\circ} 13$

Sidi Daoud - Carrières : site n ${ }^{\circ} 163$

Sidi Daoud - Corps de garde : site $n^{\circ} 161$

Sidi Daoud - Djilia : site $n^{\circ} 161$ bis
Sidi Daoud - Misua : site $\mathrm{n}^{\circ} 162$

Sidi el Khafi : site $n^{\circ} 74$

Sidi el Mehersi : site $n^{\circ} 134$

Sidi Garous : site $n^{\circ} 17$

Sidi Gherib : site $n^{\circ} 205$

Sidi Mansour : site $n^{\circ} 113$

Sidi Mansour - Henchir ech

Cheggaf : site $n^{\circ} 68$

Sidi Mechrig : site $n^{\circ} 206$

Sidi Mezara nord : site $\mathrm{n}^{\circ} 76$

Sidi Mezara sud : site $n^{\circ} 75$

Sidi Mohammed Chaouch : site $n^{\circ} 8$

Sidi Mosba - Dar ben Slimane : site $n^{\circ} 140$

Sidi Othmane : site $n^{\circ} 141$

Sidi Raïs : site $n^{\circ} 169$

Sidi Saad : site $n^{\circ} 79$

Solb ech Chergui : site $n^{\circ} 2$

Solb ech Chergui ouest : site $n^{\circ} 3$

Solb el Gharbi : site $n^{\circ} 9$

Soukrine est : site $n^{\circ} 108$

Sounine : site $\mathrm{n}^{\circ} 179$

Tabarka : site $n^{\circ} 209$

Thapsus - Rass Dimass : site $n^{\circ} 105$

Thyna : site $n^{\circ} 56$

Tobkhana : site $\mathrm{n}^{\circ} 18$

Tour Khedija : site $n^{\circ} 87$

Utique : site $n^{\circ} 174$

Vigie Rass el Koran : site $n^{\circ} 202$

Vigie Rass el Koran est : site ${ }^{\circ} 201$

Zabouza : site $n^{\circ} 45$ 
Tabl. I - Tableau chronologique de l'occupation de sites d'après la céramique recueillie

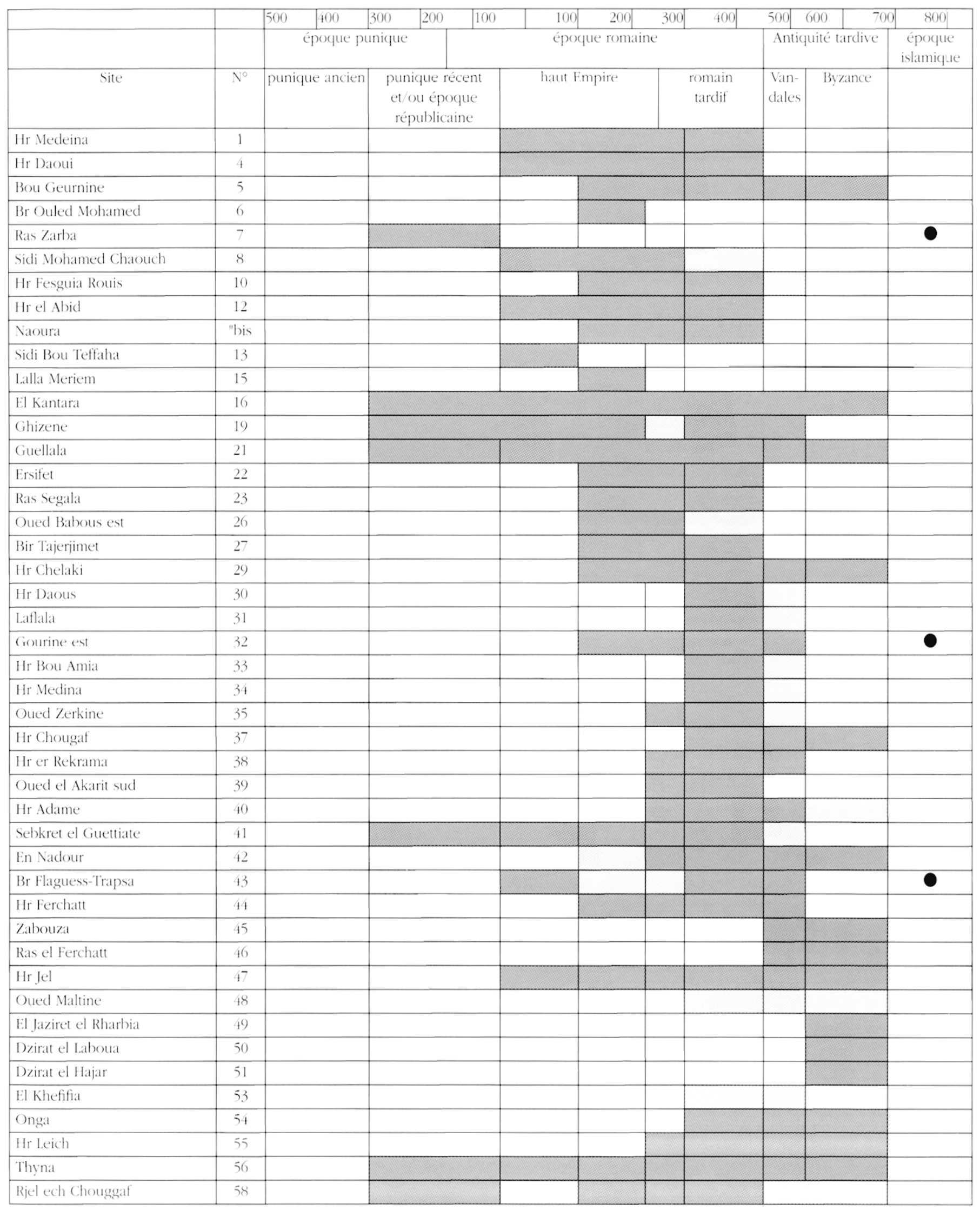


Tabl. I - Tableau chronologique de l'occupation de sites d'après la céramique recueillie (suite)

\begin{tabular}{|c|c|c|c|c|c|c|c|c|}
\hline & & \begin{tabular}{|l|l}
500 & 400 \\
\end{tabular} & 200 & \begin{tabular}{|l|l|}
100 & 200 \\
\end{tabular} & 300 & 500 & 600 & 800 \\
\hline & & époque pu & unique & époque roma & & Antiqu & lité tardive & époque \\
\hline Site & $\mathrm{N}^{\circ}$ & punique ancien & $\begin{array}{l}\text { punique récent } \\
\text { et/ou époque } \\
\text { républicaine }\end{array}$ & haut Empire & romain tardif & \begin{tabular}{|c|} 
Van1- \\
dales
\end{tabular} & Byzance & \\
\hline Ras el Berghout W & 59 & & & & & & & \\
\hline Ras el Berghout E & 60 & & & & & & & \\
\hline Borj el Hassar & 61 & & & & & & & \\
\hline Gremdi & 63 & & & & & & & \\
\hline Ras Bou Nouma & 64 & & & & & & & 0 \\
\hline Sefnou & 65 & & & & & & & \\
\hline En Ferkik est & 66 & & & & & & & \\
\hline En Ferkik ouest & 67 & & & & & & & \\
\hline Hr ech Cheggaf & 68 & & & & & & & \\
\hline El Aouabed & 70 & & & & & & & \\
\hline Hr el Majdoul & 71 & & & & & & & \\
\hline Ksar en Nouba & 72 & & & & & & & \\
\hline Hr el Flouss & 73 & & & & & & & \\
\hline Sidi Mezara sud & 75 & & & & & & & \\
\hline Sidi Mezara nord & 76 & & & & & & & \\
\hline La Louza I & 77 & & & & & & & 0 \\
\hline La Louza II & 78 & & & & & & & 0 \\
\hline Sidi Saad & 79 & & & & & & & \\
\hline La Louata & 80 & & & & & & & \\
\hline Ouled Slim & 82 & & & & & & & \\
\hline Ouled Mabrouk & 83 & & & & & & & \\
\hline Marsa Mellouleche & 84 & & & & & & & \\
\hline Rass Jezira & 85 & & & & & & & \\
\hline Jezira & 86 & & & & & & & \\
\hline Sidi Abdallah & 88 & & & & & & & \\
\hline El Alia & 90 & & & & & & & \\
\hline Borj el Mzaouak & 91 & & & & & & & \\
\hline Ghar el Dheba & 93 & & & & & & & \\
\hline Salakta sud & 94 & & & & & & & \\
\hline Rass Salakta & 95 & & & & & & & \\
\hline Mnaka sud & 96 & & & & & & & \\
\hline Mnaka nord & 97 & & & & & & & \\
\hline Douira sud & 98 & & & & & & & \\
\hline Douira nord & 99 & & & & & & & \\
\hline Sidi Ben Ghayada & 101 & & & & & & & \\
\hline Mahdia nécropole & 103 & & & & & & & 0 \\
\hline El Hafsi Neyret & 104 & & & & & & & \\
\hline Rass Dimass & 105 & & & & & & & O \\
\hline Ech Chott & 106 & & & & & & & \\
\hline Rass el Ain & 107 & & & & & & & \\
\hline Port Soukrine est & 108 & & & & & & & \\
\hline Sayada est & 109 & & & & & & & \\
\hline Lamta & 110 & & & & & & & \\
\hline Mansourah est & 111 & & & & & & & \\
\hline Mansourah ouest & 112 & & & & & & & \\
\hline
\end{tabular}


Tabl. I - Tableau chronologique de l'occupation de sites d'après la céramique recueillie (suite)

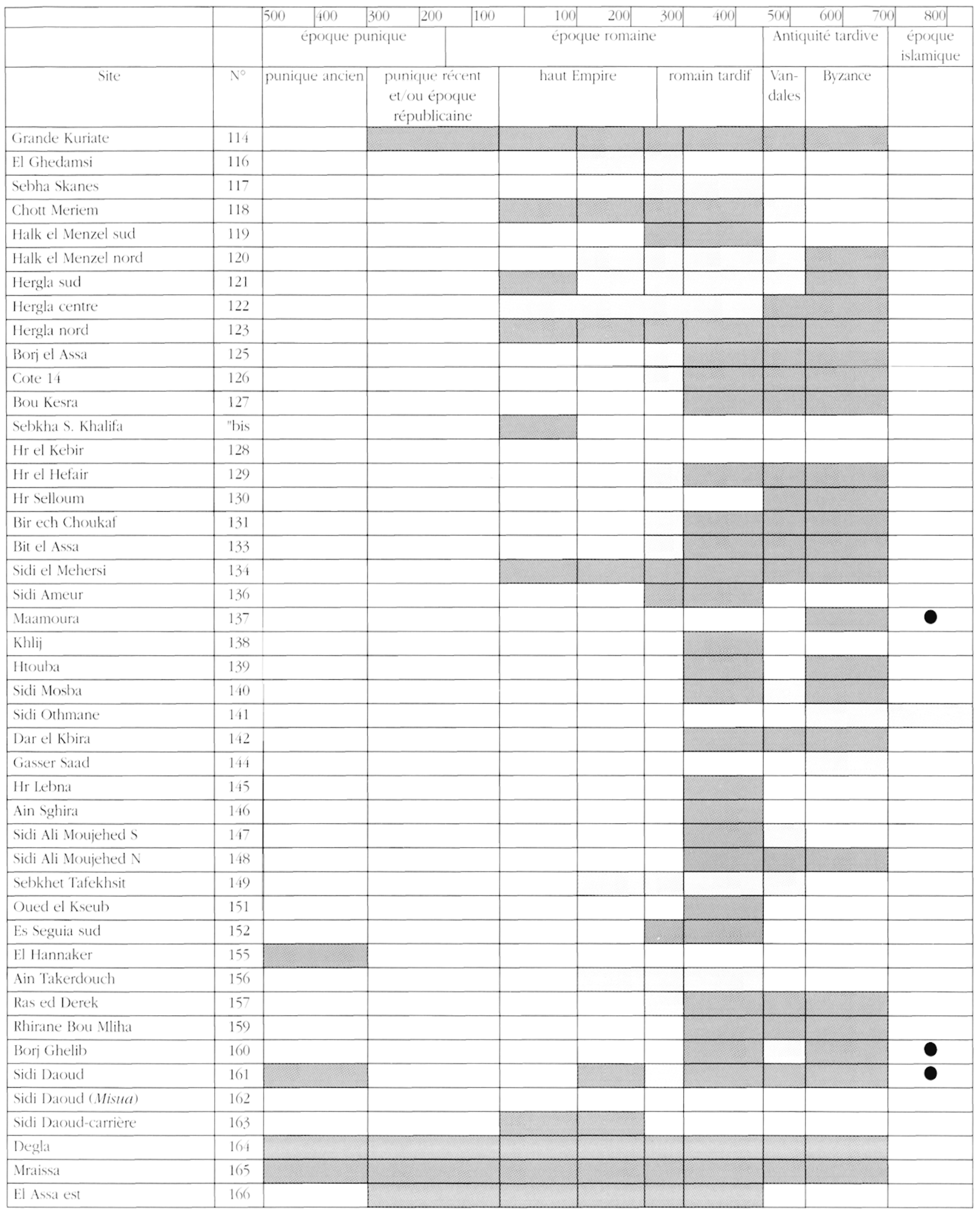


Tabl. I - Tableau chronologique de l'occupation de sites d'après la céramique recueillie (suite)

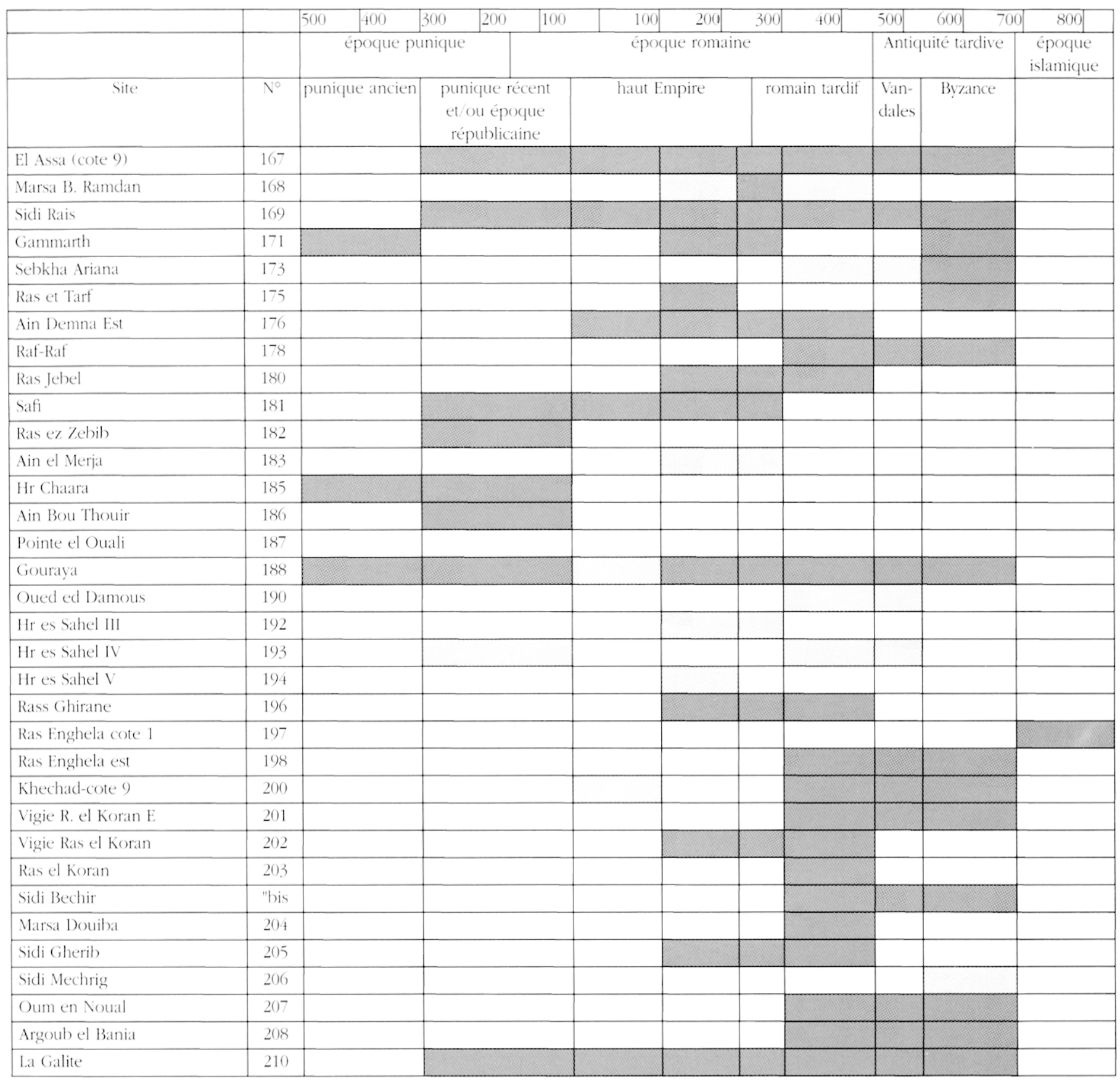


Tabl. II - Tabella riassuntiva dei principali caratteri osservati in sezione sottile. I valori persentuali variano da zero a xxxxx ; le classi dimensionali utilizzate sono le seguenti (da Capelli-Mannoni 1996) : $x$ (fine) $=<0.15 \mathrm{~mm}, \mathbf{x x}(\mathrm{medio}-\mathbf{f i n e})=0.16-0.25 \mathrm{~mm}, \mathbf{x x x}$ (media) $=0.26-0.50 \mathrm{~mm}, \operatorname{xxxx}($ medio-grossolana $)=0.51-0.75 \mathrm{~mm}, \mathbf{x x x x x}($ grossolana $=>0.75 \mathrm{~mm}$. $($ Cl. Capelli)

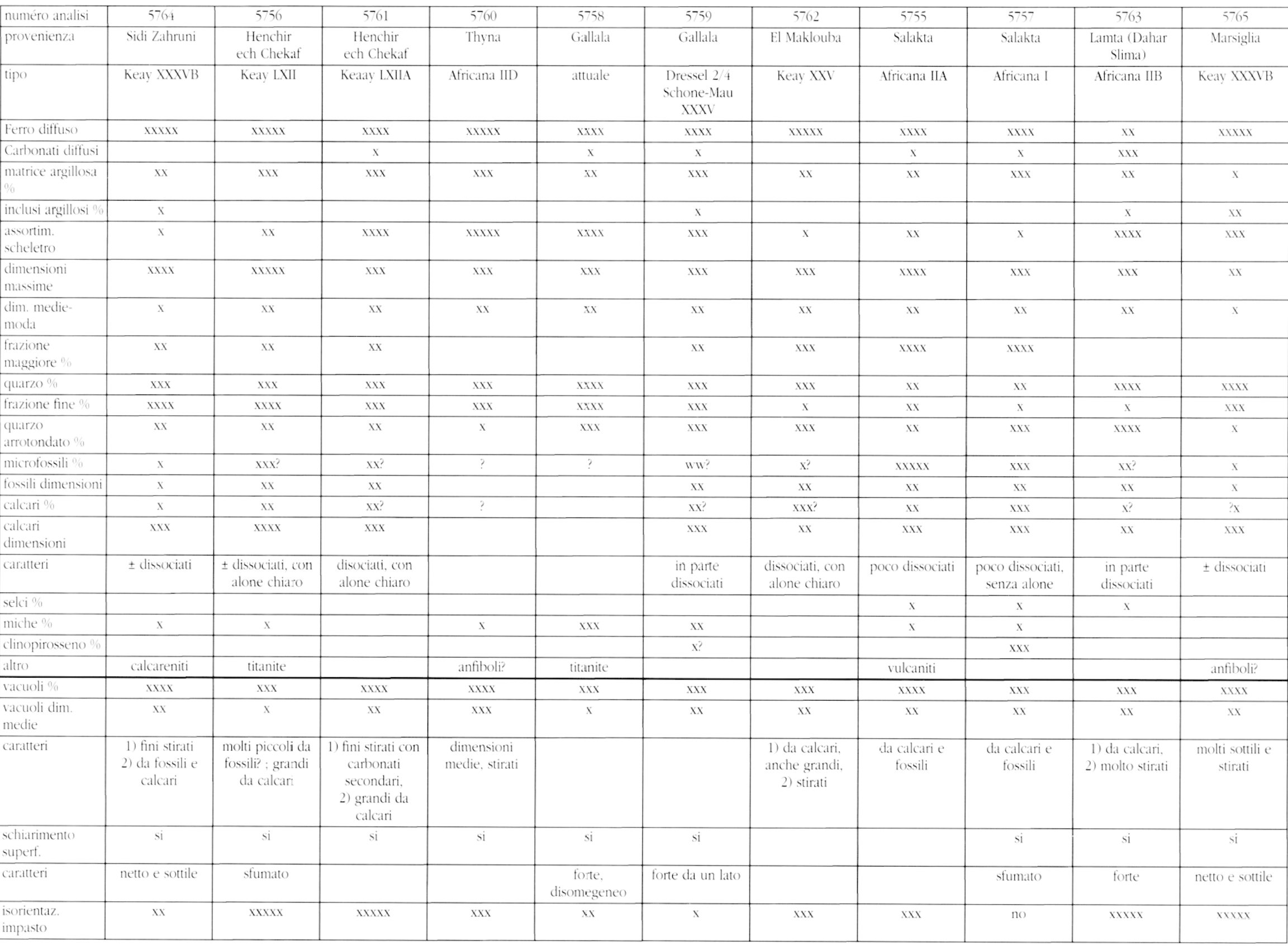

